\title{
Collective Ion Acceleration
}

\section{July 1976--September 1977}

\author{
B. B. Godfrey \\ R. J. Faehl \\ B. S. Newberger \\ W. R. Shanahan \\ L. E. Thode
}




\section{PREFACE}

In May 1977, Group T-15 was formed in the Theoretical Division of the Los Alamos Scientific Laboratory to study the physics of intense particle beams. Two principal topics of interest are collective ion acceleration and beam heating of plasmas. This report presents the results of research on collective ion acceleration performed from July 1976 through September 1977. (Prior to formulation of $\mathrm{T}-15$, beam studies were carried out in $\mathrm{T}-6$, Laser Fusion Theory.)

Section II outlines achievements of the past fifteen months. The fourteen technical appendices of Sec. V, most of which have been submitted for publication, provide detail. A tentative plan for collective ion acceleration studies during the next twelve months is proposed in Sec. III. Sec. I is a self-contained sumary of all this material. A pubilcation list is contained in Sec. IV. 
CONTENTS

PREFACE

CONTENTS

ABSTRA :I

I. SUMMARY 1

II. PROGRESS DURING FY-76T AND FY-77 6

III. PLANS FOR FY-78 16

IV. PUBLICATION LIST $\quad 22$

ACKNOWLEDGEMENTS $\quad 24$

$\begin{array}{ll}\text { APPENDICES } & 25\end{array}$

A. Computer Simulation of Collective Ion Acceleratinn 26

B. Numerical Simulation of Autoresonant Ion Acceleration 31

C. Vacuum Propagation of a Relativistic Electron Beam: 58 Equilibrium

D. Instability of a Nonneutra1 Electron Beam Propagating 114 in an Inhomogeneous Magnetic Field

E. Linear Theory of the Radially Inhomogeneous 118 ünneutralized Relativistic Electron Beam

F. Computer Simulation of Collective Ion Acceleration 149 by Discrete Cyclotron Modes

G. Relativistic Electron Beam Cyclotron Wave Growth 153 in Helical Slow Wave Structures

H. Simulation of Cyclotron Wave Growth in a Helical 164 Slow Wave Structure

I. Multidimensional Theory of the Tnhomogeneous Beam- 206 Plasma Instability

J. Langmuir Wave Phase Velocity in Unneutralized Beams 237

K. The Localized Pinch Model as a High Energy Ion 249 Col1ective Acceleration Mechanism

L. Collective: Ion Acceleration in a Travelling 262 Virtual Cáthode

M. Co1lective Ion Acceleration Through Tempora1 266 Modulation of Relativistic Electron Beam Energy

N. Initial Approaches to a 3-D Linfar Simulation Code 278 
COLLECTIVE ION ACCELERATION

JULY 1976 - SEPTEMBER 1977

by

B. B. Godfrey, R. J. Faeh1, B. S. Newberger, W. R. Shanahan and L. E. Thode

\begin{abstract}
Progress achieved in the understanding and development of collective ion acceleration is presented. Extensive analytic and computational studies of slow cyclotion wave growth on an electron beam in a helix amplifier have been performed. Research inciuded precise determination of linear coupling between beam and helix, suppression of undesired transients and end effects, and two-dimensional simulations of wave growth in physically realizable systems. Electrostatic well depths produced sxceed requirements for the Autoresonant Ion Acceleration feasibility experiment. Acceleration of test ions to modest energies in the troughs of such waves also has been demonstrated.

Smaller efforts have been devoted to alternative acceleration mechanisms. Langmuir wave phase velocity in Converging Guide Acceieration has been calculated as a function of the ratio of electron beam current to spacecharge limiting current. A new collective acceleration approach, in which cyclotron wave phase velocity is varied by modulation of electron beam voltage, is proposed. Acceleration by traveling Virtual Cathode or Iocalized Pinch has been considered, but appears less promising.

In support of this research, fundamental investigations of beam propagation in evacuated waveguides, of nonneutral beam linear eigenmodes, and of beam stability have been carried out. Several computer programs have been developed or enhanced.
\end{abstract}

Plans for future work are discussed. 


\section{SUMMARY}

Collective ion acceleration is a highly speculative, yet potentially very significant, application of intense relativistic electron beams. Eventually, it may lead to compact and economical acceleration of substantiai currents of light or heavy ions to hundreds of $\mathrm{MeV}$ per nucleon. Uses for such ion beams include controlled thermonuclear fusion, electronuclear breeding, basic nuclear physics, materials studies and radiation therapy. Increased interest in collective ion acceleration is indicated by an ever growing number of experimental and theoretical studies, both here and abroad.

In the traveling wave class of collective acceleration mechanisms, slow Langmuir or cyclotron waves are excited in the electron beam, ions are trapped in the electrostatic wells of these waves, and the waves plus ions are accelerated smoothly toward the beam velocity. The waves are grown in a reactive or resistive slow wave structure. Acceleration is achieved by spatial variation of external parameters affecting wave phase ve1ocity, such as axial magnetic field strength or metallic drift tube radius. LASL research in collective ion acceleration has followed two distinct but complementary avenues. While devoting most of our effort to specific acceleration proposals, we have also studied fundamental beam and wave phenomena relevant to the broad range of traveling wave mechanisms.

Perhaps the most basic question concerning intense relativistic electron beams is simply how large a current can propagate in an evacuated drift tube along an axial magnetic field. Although analytic estimates existed for certain limiting cases, no detailed and systematic study had ever been performed. Therefore, one of our first endeavors was to carry out an extensive numerical investigation of this issue, employing both cold fluid equilibrium models and two-dimensional computer simulations. In this way we found larger limiting currents and lower residual kinetic energies than had been expected. Moreover, the self-consistent radial profiles of beam densities and velocities, and electric and magnetic fields were obtained. For currents above the spacecharge 1imit, time-dependent virtual cathode behavior and partial beam reflection were observed.

Knowledge of realistic beam equilibria in turn permitted exact determination beam Iinear eigenmodes. ${ }^{1}$ Most striking in the resulting wave spectra were large resonance bands due to beam energy and density radial 
inhomogenlety. No normal modes in the usual sense can exist in these bands. Instead, excitation gives rise to independent oscillation of the various stream lines and decays through phase mixing. The presense of these bands eliminates most of the discrete Langnuir and cyclotron waves which vould exist in a homogeneous beam. The situation is most severe with cyclotron waves, for which only the fundamental persists and is distorted into a surface mode. A typical írequency-wavenumber spectrum appears in Fig. 1. Hov this behavior impacts collective ion accoieration is a topic of continuing research.

Among the wost promising collective acceleration proposals is Autaresonant Acceleration, which achieves acceleration of cyclotron waves by propagating the electron beam along a spatial1y diverging magnetic guide field withir a correspondingly expanding drift tube. ${ }^{2}$ Two key issues are generation of large amplitude, coherent slow cyclotron waves and, next, their stability during acceleration. The latter has been addressed in a preliminary sense first by computer simulations of the beam in the divering magnetic field. ${ }^{3}$ This study and others ${ }^{4}$ have convinced us that the beam itself is stable under these concitions. Then, we artificially launched cyclotron waves in the simulatinns and thereby successfully accelerated ions for a factor of eight gain in energy, 3

Logically, the next step was to investigate physical generation of slow cyclotron waves, and for this we chose to simulate a complete helix slow wave structure, as pictured in Fig. 2. Shown is the helix, matched terminating resistors, optional wave-absorbing graded resistances, and the amplifier cavity. We have demonstrated the importance of the matched terminations in damping large transients induced by passage of the head of the beam ty means of both simulations and analytic modeling. Unstable coupling between the slow cyclotron wave and a helix-supported wave cause wave growth. Linear growth rates are computed along the lines of the normal mode calculations described above. Finally, wave growth simulations were performed, demonstrating the amplification of cyclotron waves to strengths more than sufficient for ion acceleration. 5 Fig. 3 is a computer generated movie frame from one such simulation. During the coming year, we shall characterize the large amplitude wave itself and how it is affected by extraction from the helix, by adiabatic compression of the beam, 
and by transverse energy spread in the beam.

Converging Guide Acceleration employs Langmuir waves, accelerated in a beam propagating a]ong a constant strength magnetic field in a spatially converging drift tube. ${ }^{6}$ We have explicitly considered only one aspect of this proposal, the variation of wave phase velocity with drift tube radius. For any realistic beam in a very strong =ingetic field, the fhase velocity of the slowest Langmuir wave drops to zero only as the drift tube radius approaches the spacecharge 1imit. 7 Weaker fields give larger ainimum velocities. $^{1}$ Fig. 4 shows the typical variation of wave and beam velocity with drift tube radius. We expect to devote a continuing, low level effort to this acceleration proposal.

Recent1y, we have discovered another acceleration mechanism in which ions are accelerated by phase shifts induced in cyclotron waves by temporal variation of the electron beam injection energy. ${ }^{8}$ This procedure offers particularly short acceleration length, but at the cost of more complex. electron beam generator technology. Iiere, too, we hope to be able to devote more effort as time permits.

A traveling Virtual Cathode and a Localized Pinch in electron beams a]so were briefly considered as Jon acceleration mechanisms. 9,10 Simulation results were not encouraging.

1. B. B. Godfrey, submitted to IEEE Plas. Sci.

2. M. L. Sloan and W. E. Drummond, Phys. Rev. Lett. 31, 1234 (1973).

3. B. B. Godfrey, IEEE Plas. Sci., to be published.

4. W. R. Shanahan, Fhys. Rev. A 16, 377 (1977).

5. B. B. Godfrey, R. J. Faehl, B. S. Newberger, W. R. Shanahan, and L. E. Thode, Proc. 2nd Int. Conf. Elect. Ion Beam Res. Tech. (Corne11, 1977), to be published.

6. P. Sprangle, A. T. Drobot, and W. M. Manheimer, Phys. Rev. Lett. 29, 272 (1976).

7. B. B. Godfrey, IEEE Plas. Sci., to be published.

8. R. J. Faehl and B. B. Godfrey, submitted to Phys. Rev. Lett.

9. R. B. Miller, R. J. Faeh1, T. C. Genoni and W. A. Proctor, IEEE Nuc. Sci. 14, 1648 (1977).

10. B. B. Godfrey, IEEE Plas. Sci., to be published. 


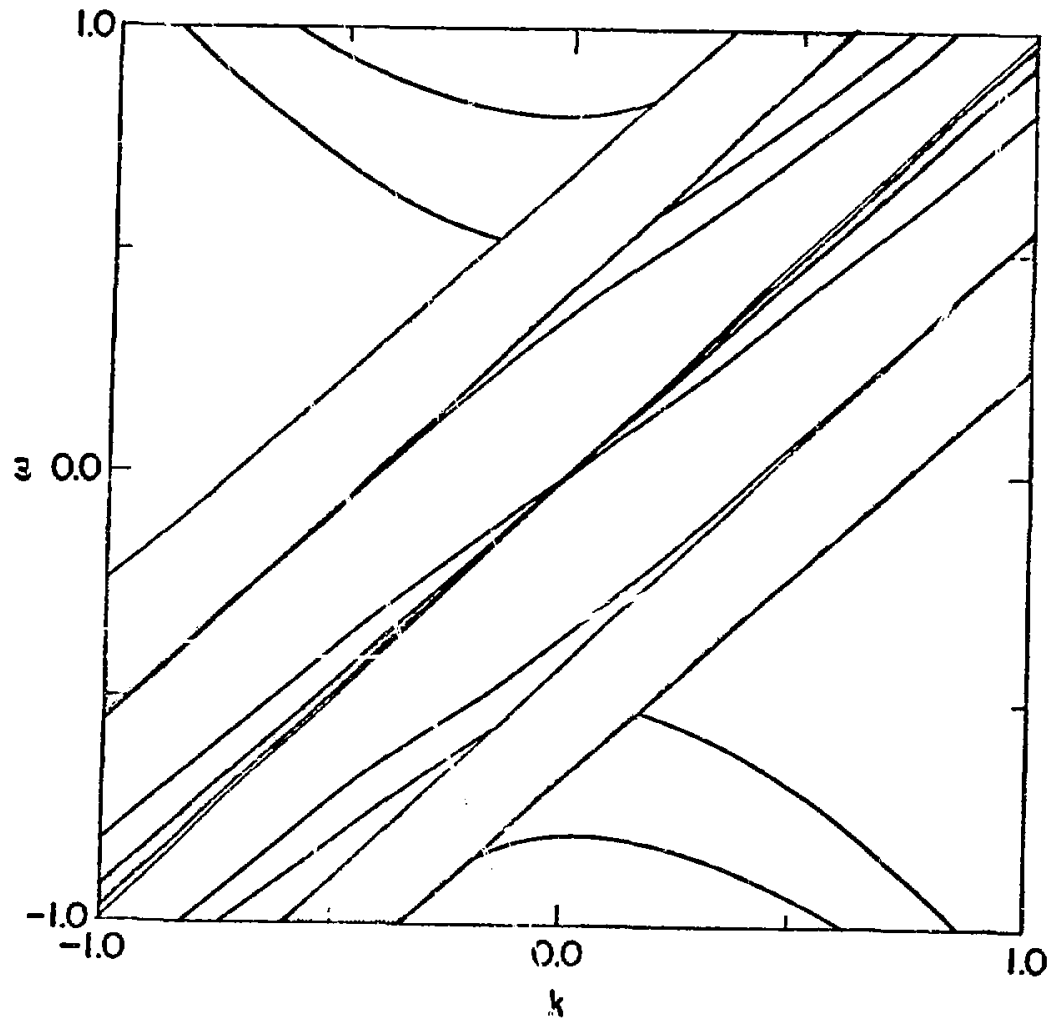

$$
\begin{array}{lc}
\nu=1.75 & R / a=2.0 \\
\gamma=7.0 & \omega_{c} / \omega_{p}=2.0
\end{array}
$$

Fig, 1. Typical frequency-wavenumber diagram for radially inhomogeneous electron beam. No discrete modes occur in shaded resonance bands.

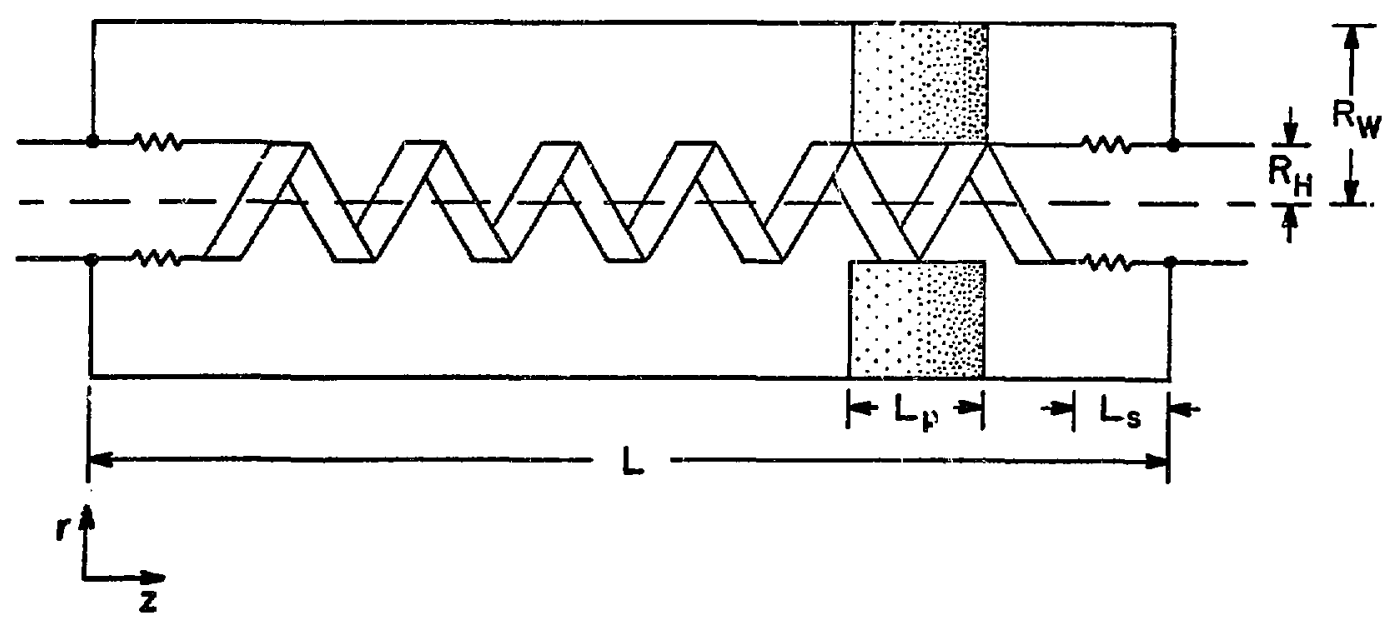

Fig. 2. Helical slow wave structure, with helix, matched terminating resistors, optional graded resistances, and cavity. 


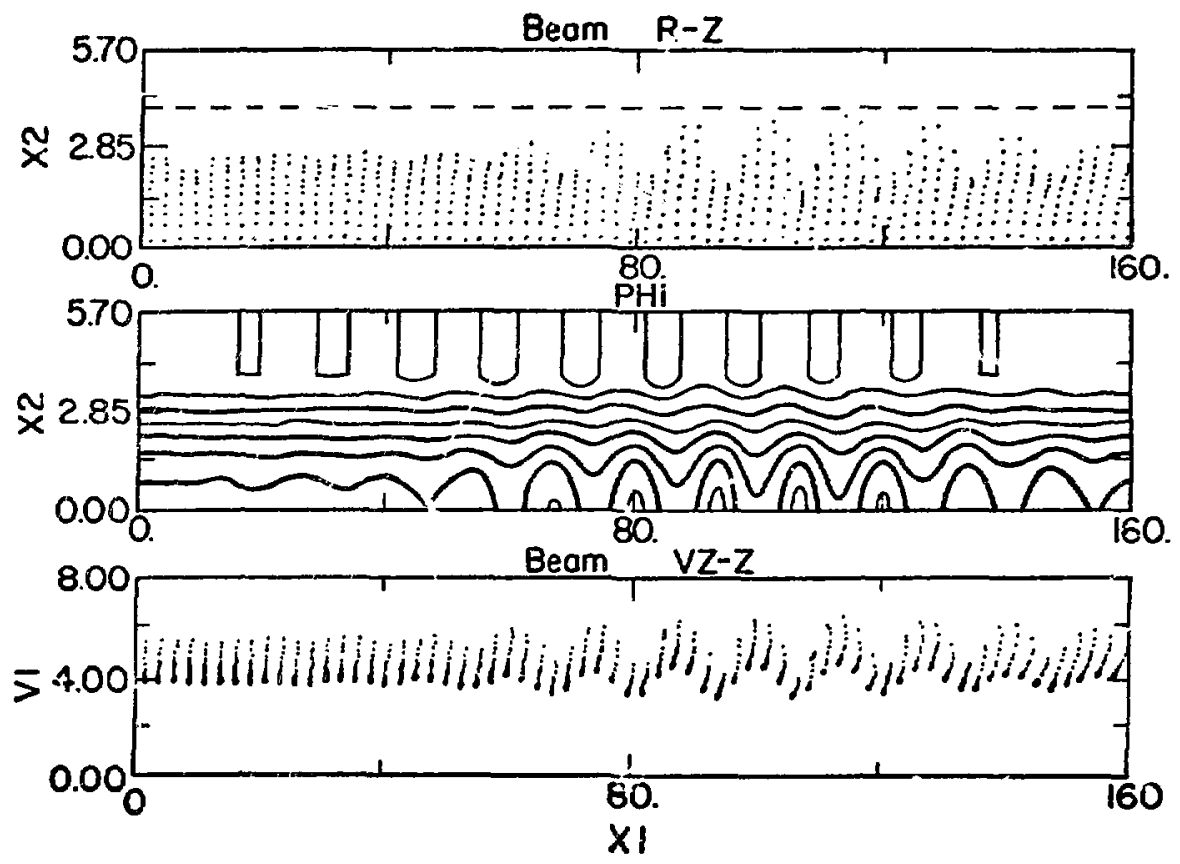

Fig. 3. Computer-generated movie frame of cyclotron wave growth simulation. Plots, top to bottom, show electrons in the amplifier cavity, contours of the electrostatic potential, and electron axial momentum vs. axial location. (Helix is at $\mathrm{X} \angle=3.8$. )

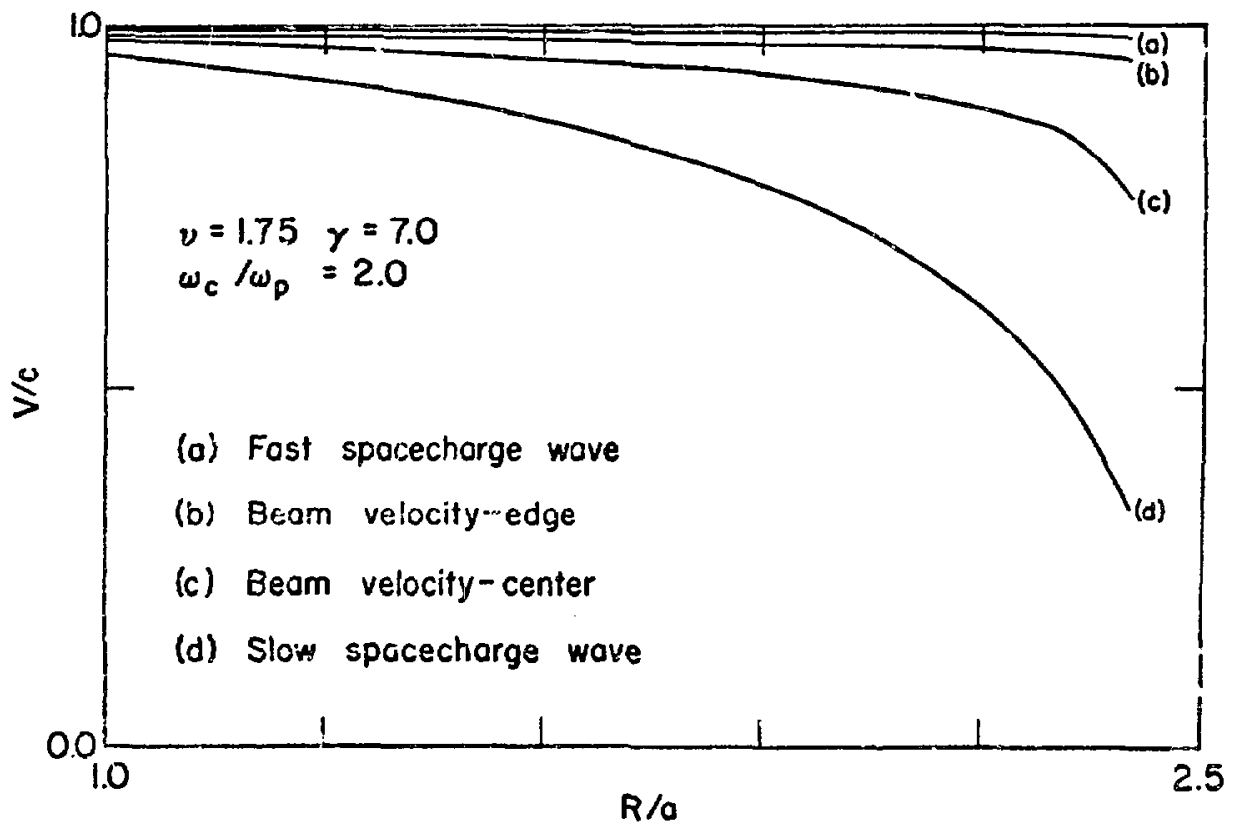

Fig. 4. Velocities of electron beam and of fundamental Langmuir waves as functions of drift tube radius. Curves terminate at spacecharge limit. 
Computational and analytic research on collective ion acceleration during the last fifteen months of LASL has been directed primarily toward guidance of the Autoresonant Acceleration feasibility experiment to be conducted by Austin Research Associates, Inc. under Army sponsorsip. In the first half of this period we addressed general questions relating to beam stability, wave propagation, and ion acceleration. More recently, we have given heavy emphasis to slow cyclotron wave growth in a helix amplifier, because it represents a first stage in the experimental program. Alternative collective acceleration proposals exist, and we have studied them as well, aithough at a lower level of effort. Among these are Converging Guide Acceleration, Traveling Virtual Cathode Acceleration, the Localized Pinch Model, and Modulated Phase Acceleration. The last recently was developed here. In parallel, we have written or enhanced several computer codes.

\section{Autoresonant Acceleration}

Our initial investigations of Autoresonant Acceleration begari prior to the current reporting period with a study of unneutralized beam transport along magnetic guide fields in both constant radius and flared metalic drift tubes. Self consistant simulations were carried out using the then recently developed two-dimensional, relativistic, electromagnetic particlein-cell code CCUBE, configured in this case for cylindrical or spherical geometry. As was hoped, the beam propagates without mishap. Moreover, beams in flared magnetic fields and drift tubes assume equilibria locally approximating those in corresponding constant radius cases. In other words expansion was verified to be adiabatic.

We then superimposed large amplitude slow cyclotron waves on the beam in our simulation by suitably modulating the upstream numerical boundary conditions. Although the waves propagate stably and at approximately the expected phase velocities, we observed a small but clear radial shear in the phase velocity. As a result waves slowly lost coherence. Nonetheless, we successfully accelerated test ions in the troughs of the waves by a factor of eight in energy over modest distances. 
Simple analytic models were developed to clarify and extend the simulation results. They indicate that the beam current to energy ratio, $\nu / \gamma$, should be in the range $1 / 4$ to $1 / 3$ for best acceleration. The potential well depth associated with a large amplitude wave is of order $2 \nu \ln \left(a_{\max } / a_{\min }\right)$ so long $\mathrm{ka}<1$. For $\mathrm{ka}>2$ wall depth decreases rapidly. In these expressions $k$ is the axial wave number and a is the local average beam radius, while a $a_{\max }$ and $a_{\min }$ are maximum and minimum radii of beam modulation. An envelope equation reasonably characterizing the large amplitude waves for as long as they remain coherent was derived. The observed phase mixing among radial stream lines was found to be due to the intrinsic radial variation of beam kinetic energy. The coherence length is such that ion energy only up to $2 \pi$ times the electron energy can be expected unles: the phase mixing problem is solved. We return to this issue below.

In addition to simulations of already formed beam equilibria, we considered the initial injection of a beam through the anode foil into the drift tube. Here, because the radial electric field of the beam is shorted at the foil whereas the azimuthal magnetic field of the beam is not, the beam pinches sharply. The pinch in turn induces a train of standing, zerofrequency cyclotron waves on the beam. Such waves, which result from any discontinuity in drift tube parameters, are highly undesirable in that they beat against traveling cyclotron waves. Note that the standing waves also are subject to stream line phase mixing. M. L. Sloan has suggested that an abrupt increase in axial magnetic field strength at the pinch location can significantly reduce the amplitude of the standing wave, although at the cost of increasing slightly the incoherent transverse motion of beam electrons. Appendices A and B provide further details. The first appendix is an internal summary of research performed through October, 1976.

Because of its importance in many collective acceleration proposals, we have carried out a separate extended background study of beam propagation near the spacecharge limit and in weak axial magnetic fields. The work involved simulations using CCUBE, numerical solutions of the cylindrical beam equilibrium equations, and analyses of experiments. Effects considered include beam expansion, beam rotation, and radial variation of beam parameters. Generally, we find that, even in the infinite guide field limit, taking account of radial variation in beam velocity gives larger 
limiting currents than had previously been determined. (Sloan and Thompson, and Proctor and Genoni also have obtained this result.) Reduced axial magnetic field strength, which permits beam expansion after injection into the drift tube, at first causes the spacecharge limit on current to rise even more. However, with further reduction in external field strength, the beam must rotate rapidly to maintain equilibrium, and the spacecharge current limit drops swiftly to zero. See Appendix C.

These results on the spacecharge current limit of rotating beams in weak axial magnetic fields are important in determining the minimum possible field, hence the maximum possible ion energy, in the feasibility experiment. The second criterion for minimum magnetic field strength is beam stability. H. V. Wong, et. al. have proven stability for solid beams provided the rotation frequency is less than the speed of light divided by the drift tube radius. However, our recent preliminary numerical analysis of the same problem, also described in Appendix $C$, suggests that the beam typically remains stable even at somewhat higher rotation rates. Both Wong's analysis and our extension apply only in uniform radius geometry. In the actual experiment, the beam, magnetic field, and drift tube all flare adiabatically as a function as axial position, giving rise to an axial shear in rotation rate. In Appendix $D$ we examine this situation and find that it too does not lead to instability.

In order to understand loss of radial phase coherence by cyclotron waves in the computer simulations described above, we have derived and studied intensely the exact equations describing the normal modes of cold, unneutralized beams. Our results, as they bear upon cyclotron waves, are striking. First, in contrast to the predictions of homogeneous beam models, there is only one discrete slow cyclotron mode. Second, this wave is strongly distorted radialiy and has the character of a surface mode. Third, a continuum band of singular cyclotron eigenmodes exists, and corresponds to independent oscillations of the various radial streamiines. It is now clear that the waves excited in the early simulations rere packets of singular eigenmodes, the phase mixing and decay of which is well explained by our model. Moreover, due to the wide band width of the singular spectrum relative to that of the discrete mode, the former will almost always be excited by an external driver. We must, therefore, rely on an instability mechanism to generate the discrete mode. Thether the sharp peaking of the 
discrete wave at the beam surface is undesirable awaits further analysis. Appendix E gives both specific calculations and general features of the unnuetralized, inhomogeneous beam eigenmode spectrum.

The linear growth rates for cyclotron waves on beams propagating through reactive or resistive cavities also can be determined exactly with the mode1. Appendices F, G, and $H$ describe our analysis of the helical slow wave structure for cyclotron wave growth. Computations predict that growth adequate for the requirements of the feasibility experiment can be achieved over a meter or so. However, the radial profile of the discrete cyclotron wave within the helical amplifier is distinctly different from its profile in a matched drift tube. Thus, care wust be taken in bringing the wave out of the amplifier to avcid loss of coherence or dumping of energy into other modes. We have also investigated cyclotron wave growth in a resistively lined drift tube. The results agree with those of Austin Research Associates, Inc, and so are not reproduced here.

Encouraged by the predictions of linear theory, we performed CCUBE simulations of cyclotron wave growth and saturation in a $15^{\circ}$ pitch angle helix. In the first series, the simulations were initialized with beam and helix already in equilibrium, so that transients could not mask the growth we wished to study. With growth from beam fluctuations, wares were observed to develop slowly and had an envelope irregular in both space and time. On the other hand, when the beam entered the helix with a $1 \%$ radial modulation at the desired frequency, waves grew at the expected rate and had a narrow bandwidth. In fact, the second mode of operation will be employed experimentally. That wave growth fiom fiuctuations was comparatively unsuccessful is good: Waves not excited to small amplitude upstream of the helix should be unable to compete with the desired wave in the helical amplifier. Using the $3.4 \mathrm{~kg}$ guide field planned for the experiment, we find the following dimensions to be optimal, beam radius of $2.65 \mathrm{~cm}$, helix radius of $3.8 \mathrm{~cm}$ and outer waveguide radius of $5.7 \mathrm{~cm}$. A length for the amplifier of about $100 \mathrm{~cm}$ is adequate to grow waves with potential wells of order $0.5 \mathrm{MeV}$, more than required by the initial experimental design. Corresponding field strengths at the helix of $40 \mathrm{kV} / \mathrm{cm}$ should not cause difficulty. However, breakdown on the helix 
due to high axial fields may prove to be a problein in larger future systems. See Appendix G.

Because the helix is a reactive element, transients induced hy the transit of the head of the beam cause the helix to ring throughout the the beam pulse, leading to virtual cathode formation and severe beam distuption, unless suitable preventive action is taken. Nor can steady state return current flow in the helix be tolerated, or the resultant modification of the axial magnetic field excites standing cyclotron waves. We have studied the effects of various resistors in parallel and in series with the helix using CCUBE in the "slug" mode: The beam is treated as a noving rigid rod of charge, while the response of the electromagnetic fields in the helical amplifier is computed exactly. The procedure is inexpensive and prevents masking of the helix behavior by beam dynamics. In this way we verified that a matched resistive termination at each end of the helix effectively damps both transients and return current in a little more than two helix wave transit times, about 25 ns for the parameters listed above. Incidently, matched terminations probably would not work as well, if the outer waveguide radius were much larger than the helix radius. With both wave growth and helix transients separately in hand, we have just completed a brief second series of particle simulations in which a beam propagated through a complete, physically terminated, helix amplifier, and large amplitude cyclotron waves grew up. Thus, the wave growth issue is almost completely resolved. Coherence and stability of the extracted wave will. be addressed in the near future. Appendices $F$ and $G$ give greater detail.

Any low density plasma in the drift tube, for instance in the ion trapping region, may interact with the electron beam via two-stream instabilities. Although we have not explicitly considered this situation, we have at hand a large body of relevant theory developed for a separate beam-plasma interaction program, which can quickly be brought to bear on the feasibility experiment, if required. A recent and particularly interesting study of two-stream instabilities in density gradients is described in Appendix I.

\section{Alternative Approaches}

Converging Guide Acceleration is so named because it employs a constant radius beam propagating in a drift tube of axially decreasing 
radius. This spatial variation of the ratio of waveguide to beam radii increases the kinetic energy of the electrons, at the expense of potential energy, and thereby accelerates Langmuir waves ard any ions trapped in them. Initial trapping of ions is, of course, facilitated by very low wave phase velocity at the upstream end of the accelerator. Appendix $\mathrm{J}$ proves that, for relativistic beams of almost any radial density profile propagating along infinitely strong axial magnetic fields, Langmuir wave phase velocity drops to zero only as the drift tube radius is increased to the spacecharge limit. The effect of weaker axial magnetic fields can be obtained numerically by employing the inhomogeneous beam linear theory inodel described previously. For progressively weaker fields, the minimum phase velocity is found to be systematically larger. It may be, therefore, that a practical Converging Guide Accelerator will require ions injected with energies of $20 \mathrm{MeV}$ or so. On the other hand, Converging Guide Acce1eration may prove more effective than Autoresonant Accelration in acce1erating ions at very high energy. It should also be noted that, in contrast to cyclotron waves, Langmuir waves are not distorted into surface modes by beam energy radial inhomogeniety. Application of our numerical linear theory code to Langmuir waves is contained in Appendix $E$.

The Localized Pinch Model is the oldest traveling wave collective ion acceleration proposal. The electron beam propagates not in vacuum but through a low density ion background, $n_{i} / n_{e}=\gamma^{-2}$ 。 This is Budker's force neutral equilibrium condition, and requires no axial guide magnetic field. The beam does not rotate. According to the model, if a local excess of ions occurs, the bean pinches just downstream of the ion clump, and the resulting axial electric field ther. accelerates the clump. Moreover, because the pinch always must be diwnstream of the clump, the process is self-synchronous and should persist over long distances. We have completed a brief snalytic and numerical study of the Localized Pinch Model, the results of which are in Appendix $\mathbb{N}$. Basically, we find that the localized pinch and electrostatic well do form just downstream of a clump of excess ions and that the ion clump and pinch do, at first, accelerate together. However, the configuration is phase unstable: The ion clump spreads axially, the pinch weakens, and acceleration ceases before the ions can 
achieve more than a few times the electron kinetic energy. We conclude, therefore, that, although the Localized Pinch Model may indeed be responsible for some low energy ion acceleration observed in low density gas beam transpost experiments, it is not a viable mechanism for obtaining high ion energies.

In collaboration with the Air Force Weapons Laboratory, we have carried out a modest simulation of the Traveling Virtual Cathode concept. A beam of uniform radius propagates along a strong guide magnetic field in an evacuated metal drift tube of spatially increasing radius. At some point the ratio of drift tube to beam radii exceeds the spacecharge limit, and a virtual cathode forms. In principle, the virtual cathode can be moved about axially by changing the injected beam current or voltage. Ions trapped in the associated potential well would be accelerateu. However, simulations indicate that the actual motion of the virtual cathode is erratic and difficult to control. Reflected electrons cause the virtual cathode to move rapidly upstream even with constant beam current and voltage. Moreover, two-stream instability between the primary and reflected beams superimposes irregular changes in the depth and location of the electrostatic well. Employing a hollow beam and a weak guide field seems to help somewhat. Research is continuing at a low level. Appendix L provides a summary of early results.

Finally, we mention a novel collective ion acceleration scheme, tentatively named Modulated Phase Acceleration, which recently was developed at Los Alamos Scientific iaboratory. It resembles Autoresonant Accleration in that is employs slow cyclotron waves on a nonneutral electron beam. However, whereas Autoresonant Acceleration employs a spatial variation of beam and drift tube radii to accelerate ions, our new proposal employs tempora1 variation of the beam energy. A programed sweep of the beam injection energy produces a corresponding phase shift in the cyclotron waves, which accelerates ions trapped in them. Our limited investigations of this idea, containza in Appendix $M$, indicate that for comparable electron beam parameter ranges, shorter ion acceleration lengths can be achieved than for a1ternate traveling wave methods. On the other hand, an untested eiectron beam generator technology is required. We shall further develop the concept as time permits. 
Code Development

Our principle numerical tool is CCUBE, a two-dimensional, relativistic, electromagnetic, plasma simulation code. Some of its many applications have been described in preceding paragraphs. Aithough the computer program has never been formally documented, summaries of its principle features are given in Appendices $A$ and $C$. CCUBE stands out from other particle-in-cell simulation codes in two important respects: It can simulate beams in any separable, orthogonal coordinate system, and it is comparatively free of common numerical instabilities. Flexibility in the treatment of particle and field boundary conditions and in the display of results also is important.

The improved accuracy and numerical stability of CCUBE is due to the canonical momentum algorithm used in determining the trajectories of simulation particles. This numerical algorithm requires elcctromagnetic potentials instead of fields. Thus, the program as originally written computed directly the scaler and vector potentials, and only generated fields as needed for diagnostics. However, midway through the reporting period we completely rewrote the field-potential solver to determine the electromagnetic fields directly and from them to obtain potentials for advancing the particles. In this way, the capability of the code to hanile reactive and resistive structures within the simulation mesh was significantly enhanced. The modified program aiso explicitly separates the function of enforcing the equation of continuity on the charge and current densities from the function of advancing the fields and potentials. We now have a direct measure of interpolation errors involved in obtaining these source densities from the particle positions and velocities. The corrections performed on the currents are of order a few percent.

After completing the new field-potential solver, we developed the special package needed for simulating a complete helix amplifier, including helix, resistive terminations, and metal cavity. Part of the package is the provision for replacing the simulation particles by a rigidly moving charged ind, if desired, for economical studies of amplifier response. Also developed were special wave absorbing boundary 
conditions. All told, the helix amplifier studies represent some of the most elaborate plasma simulations ever performed.

The program written for determinirg the eigenmodes and eigenfrequencies of realistic cold beam equilibria is GRADR. The application of this code to studies of the Langmuir and cyclotror wave spectra were described earlier. It allows, in particular, the proper treatment of beam rotation and radial inhomogeniety. GRADR functions as follows. A cold fluid cylindrical beam equilibrium is describable as three beam and three field quantities, each a function of radius only. Maxwell's equations plus the beam equations of motion provide four relations among the six quantities. Two more relations are: required to specify an equilibrium. GRADR permits the user to define these constraints as desired. Typical choices are uniformity of current and cotal energy arross the beam. With the two relations given, GRADR solves the six equations iteratively to obtain a self-consistent equilibrium.

The user is then free to compute, either interactively or automatically, the linear eigenmodes and wave frequencies consistant with the equilibrium. The eigenmodes are defined by four linear differential equations, which are solved by the Runge-Kutta method. Frequencies are determined iteratively using Muller's method. The choice of boundary conditions permits investigation of beam stability in drift tubes, resistive liners, helices, and other structures of interest. Appendix E describes the code in detail and gives illustrative output. Only several minutes of CDC-7600 computer time are required to generate frequencywarenumber dispersior plots covering a large region of waverumber space. GRADR also evaluates the order and location of branch points in these diagrams.

Although GRADR has proven to be an invaluable tool in studying beam stability and wave growth, there are important cases which it cannot treat. Consider, for instance, determining numerically the stability of large amplitude cyclotron waves on an expanding beam to non-axisymmetric perturbations. We have, therefore, initiated a low level effort to develop a three-dimensional linear theory code to handle such problems. Our first attempt, based upon a linearized particle-in-cell approach, is discussed 
in Appendix N. After careful consideration, we have abandoned that technique as too expensive in both computer time and memory reciuirements. In essence, such a computer colle would consume nearly the same resources as a three-dimensional extension of CCUBE while producing less useful results. Recently, we have given attention to a less ambitious but hopefully more practical approach, namely a three-dimension linearized fluid code. The necessary algorithms have been developed and will be implemented during the coming year.

Several smaller computer prograns have been written to solve specific problems, including ion trapping and acceleration for Modulated Phase Acceleration (Appendix M), cyclotron wave nonlinear envelope equations (Appendix B), stability of a beam with axially varying rotation frequency (Appendix D), and two-stream instability in an inhomogeneous plasma (Appendix I). NOVA, the first of these, may also be of use in considering the effects of beam energy ripple for other ion acceleration mechanisms. 
The Autoresonant Acceleration reasibility experiment may conveniently be divided into two parts. In the first, the electron bean equilibrium is established, large amplitude slow cyclotron waves are grown, and the beam is compressed adiabatically to reduce wave phase velocity. Ions are trapped in the electrostatic wells of the waves, and the beam is expanded adiabatically to accelerate the ions, in the second half. Throughout the coming year we shall devote most of our effort to investigating in detail all aspects of the first part in order to provide imely guidance of the experiment. As time permits, we also sha11 study basic features of alternative collective ion acceleration mechanisms. Code development vill continue to receive considerable attention. The programs CCUBE and GRADR will be enhanced, and a new three-dimensional linear theory program will. be developed. A tentative schedule of activities is provided at the end of this section.

\section{Autoresonant Acceleration}

Although oux study of slow cyclotron wave growth in a helix amplifier is for the most part complete, we shall spend a few months tying up various details. In particular, helix simulations ac a pitch angle of $8^{\circ}$ will be performed in order to approximate more closely revised experimental plans. Results are expected to be qualitatively similar to those of our $15^{\circ}$ pitch angle investigations but with growth lengths and time scales about twice as long. In these simulations the helix will be driven at its upstream end by an RF power supply tuned to the frequency of the desired cyclotron wave. This more realistic method for exciting the helix will replace the $1 \%$ radial premodulation on the beam employed last year.

As described in Sec. II, the radial profiles of the linear cyclotron mode are distinctly different in the helix and in a drift tube of equal radius. We shall, therefore, give special attention to extracting the cyclotron wave from the helix amplifier in such a way that coherence is not degraded. How difficult the extraction problem will be, and what special techniques (if any) are required cannot be estimated realistically at this time. Coherence and stability of the wave will be tested by allowing it to propagate a distance of several wavelengths in a constant radius drift tube. 
Because it is not clear whether a resonant loop amplifier might be superior to a helix amplifier, we shall perform a parallal study of the resonant loop system. The investigation should proceed comparativejy rapidly, since many of the associated computational and physical prpblems are shared by the helix system and wili already have been solved. It will, however, be necessary to upgrade GRADR somewhat in order to obtain linear results helptul in planning the CCUBE simulations.

By spring we expect to have completed wave excitation, growth, and extraction simulations for both the helix and resonant loop amplifiers. At that time we shall decide which is more promising and drop the other from further: consideration. We then shall perform analytic and numerical studies of cyclotron waves grown by the prefered slow wave structure in order to characterize the nonlinear behavior. An analytic model will be developed, if possible. At the least, the nonlinear amplitude, wavelength, phase velocity, and group velocity will be determined numerically as a function of axial magnetic field strength. The results of this study will bear heavily on the course of research for the next year.

Following characterization of the waves in a constant radius drift tube, we shall simulate adiabatic compression of the electron beam plus cyclotron waves. Although no physical difficulties are anticipated in this stage, it is prudent that we study it anyway. If the work progresses rapidly, we may have time to investigate ion loading into realistic cyclotron waves before the close of the fiscal year.

The planned analytic and computational program described up to this point will have treated only electron beams with negligable transverse energy. Due to foil scatter and other diode effects, real beams always exhibit some transverse energy spread. In the feasibility experiment at least a $7^{\circ}$ scatter is likely before compression, and about three times that amount after. Therefore, in parallel with the wave characterization and beam compression studies in the second half of the year, we shall systematically reproduce for the system of choice all the simulations performed with a cold beam, but with realistic transverse energy scatter included. The two most important phenomena to be evaluated are nodification of the nonlinear cyclotron wave radial prufile and damping or coherence loss in 
the waves. If all goes well, we shall have obtained a fairly complete picture of the entire front half of the experimental accelerator and, in addition, produced a large body of valuable basic knowledge.

\section{Alternative Approaches}

Because we intend to devote the vast majority of our resources to Autoresonant Acceleration, we are not allocating time specifically to alternative collective ion acceleration mechanisms. Nonetheless, we hope to be able to consider aspects of these other approaches now and again. Two short research topics in Converging Guide Acceleration suggest themselves, computing Langmuir wave minimum phase velocity as a function of magnetic field strength and designing a CGA experiment for the Army's $3 \mathrm{MeV}$ electror bear generator at Austin Research Associates, Inc. Col1aboration with Cornel1 University and the Nava1 Research Laboratory on Converging Guide Acceleration studies also may prove practical.

Although computer study of Traveling Virtual Cathode ion acceleration is now principally in the hands of the Air Force Weapons Laboratory, we shall continue to provide assistance as needed. Our Modulated Phase Acceleration research will be directed to minimizing the required variaticn in electron beam energy.

Code Development

Several significant internal modifications of CCUBE are planned for the upcoming fiscal year. These changes will have the effects, first, of further improving the accuracy and stability of the code and, second, of enhancing its flexibility in treating complex geometries. The portabilicy of the code and the appearance of its output also will be improved. Changes affecting portability are a major step toward moving CCUBE from the CDC-7600 to the larger and faster Cray-1 computer. Eventually, use of the latter machine may permit modest three-dimensional numerical simulations of beam phenomena.

It was noted in Sec. II that CCUBE employs electromagnetic potentials rather than fields in determining particle trajectories, and in this way avoids certain common numerical instabilities. We have developed a spacetime-symmetric Galerkin finite element algorithm which appears to have 
equal or better stability properties and uses electromagnetic fields directly. Moreover, the algurithm guarantees preservation of the equation of continuity in the current interpolation process and decreases energy conservation errors in the presence of large magnetic fields. Computer memory requirements will be reduced about $30 \%$, while running time should remain roughly the same. Because solution of Poisson's equation will no longer be necessary, CCUBE will be able to handle non-separable coordinates with minor additional modifications. Notwithstanding the great promise of our new algorithm, a note of caution is appropriate: The modifications represent a severe departure from conventional techniques in several respects and may, therefore, contain hidden difficulties. We shall take care to test CCUBE in detail at each step of the implementation.

If the new algorithm proves successful, we shall next develop and implement a forth order correction procedure to reduce numerical dispersion in light waves. The change is not necessary at the present time, but may become important for simulation of ion acceleration to relativistic energies. Lat: in the year, we shall convert CCUBE for compatibility with the standard Extended Fortran Compiler and the Common Graphics System. An exact date for this activity is difficult to estimate, since it depends on software deve1opment by the LASL computer center staff. It is conceivable, but not 1ikely, that transfer of CCUBE to the Cray-1 computer could begin before the end of the fiscal year.

GRADR, the relativistic electron beam linear dispersion computer code, can at present treat only cylindrically symmetric problems, i.e., only those for which the beam equilibrium and boundary conditions are axially and rotationally invarient. Early this year we intend to enhance the program to accept axially and rotationally periodic boundary conditions. Our immediate motivation is to examine in more detail the resonant loop cyclotron wa : amplifier described above, although other interesting cases will come within our grasp as well.

We shall con:inue at a low level our effort to develop a time dependent, three dimensional linear theory code along the lines discussed in Sec. II. At issue is not so much whether such a code can be developed as whether it will be economical to use. Various short, special purpose conputer programs will be written, as needed. 
Tentative Schedule

A tentative schedule of our plenned activities for FY-78 is 1isted by quarters in the Figure on the next page. The anticipated level of effort is 2.5 to 3.0 man-years. We emphasise that the outlined research program is exploratory in nature and, therefore, subject to change as results unfold. 


\section{FY-78 TENTATIVE SCHEDULE}

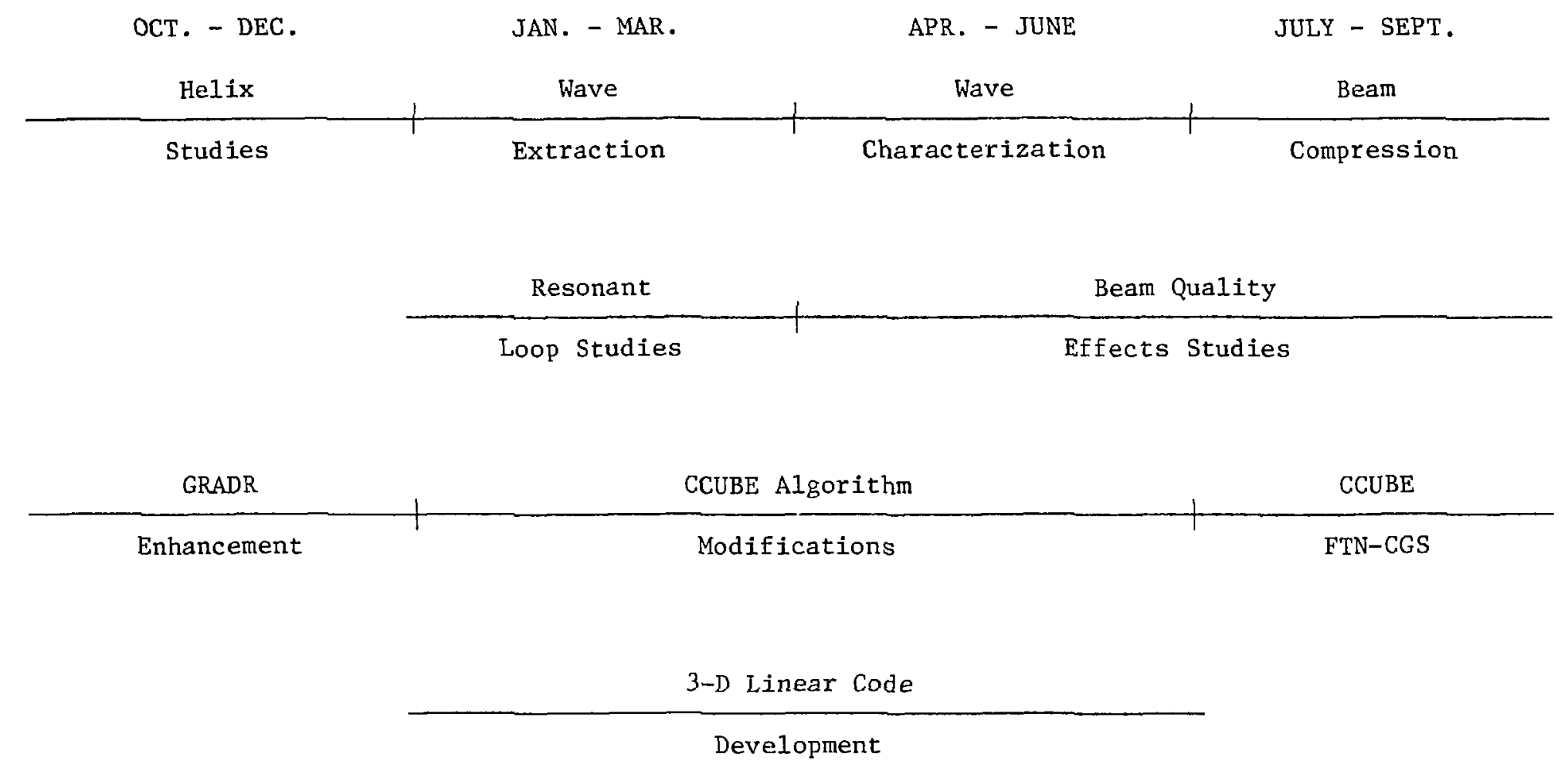




\section{Publication List}

B. B. Godfrey and W. R. Shanahan, "Computer Simulation of Collective Ion Acceleration," Los Alamos Scientific Laboratory, Theoretical Division on Annual Report July 1975-September 1976, LA-6816-PR.

R. J. Faehl, R. B. Miller, ${ }^{\dagger}$ and B. B. Godfrey, "Simulation of Virtual Cathode Formation During Vacuum Propagation", Bull. Amer. Phys. Soc. 21, 1165 (1976).

B. B. Godfrey, "Collective Ion Acceleration in Nonneutral Relativistic Electron Beams", Bull. Amer. Phys. Soc. 21, 1183 (19,76).

W. R. Shanahan, "Instability of a Nonneutral Electron Beam in an Inhomogeneous Magnetic Field", Bull. Amer. Phys. Soc. 22, 129 (1977).

W. K. Shanahan, "Inhomogeneous Beam-Plasma Instability in One and Two Dimensions". IEEE Conference Record - Abstracts, The IEEE 1977 International Conzerence on Plasma Science, 162 (1977).

B. B. Godfrey, R. J. Faehl, W. R. Shanahan, and L. E. Thode, "Computer Simulation of the Localized Pinch Model of Collective Ion Acceleration", IEEE Conference Record - Abstracts, The IEEE 1977 International Conference on Plasma Science, 174 (1977).

W. R. Shanahan, "Instability of a Nonneutral Electron Beam Propagating in an Inhomogeneous Magnetic Field", Phys. Rev. A 16, 377 (1977).

R. J. Faehl, B. B. Godfrey, B. S. Newberger, and W. R. Shanahan, "Computer Simulation of Collective Ion Acceleration by Discrete Cyclotron Modes," IEEE Trans. on Nuclear Science NS-14, 1637 (1977).

R. B. Miller,$^{\dagger}$ R. J. Faehl, T. C. Genoni, ${ }^{\dagger \dagger}$ and W. A. Proctor, ${ }^{\dagger \dagger}$ "Collective Ion Acceleration in a Travelling Virtual Cathode", IEEE Trans. on Nuclear Science NS-14, 1648 (1977).

B. B. Godfrey, "Numerical Simulation of Autoresonant Ion Acceleration", IEEE Trans. on Plasma Science (to be published).

B. B. Godfrey, "Langmuir Wave Phase Velocity in Unneutralized Beams", IEEE Trans. on Plasma Science (to be published).

B. B. Godfrey, "The Localized Pinch Model as a High Energy Ion Collective Acceleration Mechanism", submitted to IEEE Trans. on Plasma Science.

B. B. Godfrey, R. J. Faehl, B. S. Newberger, W. R. Shanahan, and L. E. Thode, "Relativistic Electron Beam Cyclotron Wave Growth in Helical Slow Wave Structures", to be presented at the 2nd Internatioual Topical Conference on High Power Electron and Ion Beam Research and Technology, Cornell Univ., October 1977, LA-UR-77-2205. 
R. J. Faehl, B. S. Newberger, B. B. Godfrey, and W. R. Shanahan, "Simulation of Cyclotron Wave Growth in a Helical Slow Wave Structure", to be presented at the 19th Annual Meeting, American Physical Society Division of Plasma Physics, Atlanta, GA, November 1977, LA-UR-77-1773.

B. S. Newberger, R. J. Faehl, B. B. Godfrey, and W. R. Shanahan, "EncEffects in Cyclotron Wave Growth in a Helical Slow Wave Structure", to be presented at the 19 th Annual Meeting, American Physical Society Division of Plasma Physics, Atlanta, Gí, November 1978, LA-UR-77-1774.

R. J. Faehl and B. B. Godfrey, "Collective Ion Acceleration through Temporal Modulation of Relativistic Electron Beam Energy", submitted to Phys. Rev. Letters.

B. B. Godfrey, "Iinear Theory of the Radialiy Inhomogeneous Unneutralized Relativistic Electron Beam", submitted to IEEE Trans. on Plasma Science.

L. E. Thode, B. B. Godfrey, and W. R. Shanahan, "Vacuun Propagation of a Relativistic Electron Beam: Equilibrium", in preparation.

W. R. Shanahan, "Multidimensional Effects in the Inhomogeneous Beam-Plasma Instability", in preparation.

R. J. Faehl, B. S. Newberger and B. B. Godfrey, "Simulation of Cyclotron Wave Growth in a Helical Slow Wave Structure", in preparation.

$\dagger_{R}$. B. Miller is at Sandia Labs, Albuquerque.

$\dagger_{\text {T. }}$ C. Genoni and W. A. Proctor are at AFWL, Kirtland AFB. 


\section{ACKNOWLEDGEMENTS}

This document describes research performed during FY-76T and FY-77 by Group T-15, Intense Particle Beam Theory, of the Los Alamos Scientific Laboratory on collective ion acceleration in intense relativistic eiectron beams. The work was sponsored by the U. S. Army Ballistic Missile Defense Advanced Technology Center, by the Air Force Weapons Laboratory, and by the U. S. Energy Research and Development Adminiscration. We are indebted to each of these agencies for their support.

Special thanks are due to program managers at the three organizations, who provided both technical and edministrati: guidance. Dr. Larry Havard, assisted by Dr. Edward Wilkenson, supervised the Advanced Technology Center's program. Project officer at the Air Force Weapons Laboratory was first Dr. Bruce Miller and next Dr. David Straw. A grant from ERDA's Division of Physical Research was administered by Dr. William Hermannsfeldt and Dr. David Sutter. Funding from ERDA's Division of Military Applications was provided through the LASL Supporting Research prograin.

Within the Los Alamos Scientific Laboratory, Dr. Thomas Hayward coordinated efforts supported by the Ba]?istic Missile Defense Advanced Technology Center. Prior to the formation of Group T-15 in the spring of 1977, particle beam studies were carried out within Group T-6, headed by Dr. Dale Henderson. This report was prepared with the assistance of Mrs. Nathana Haines, Group Secretary. 


\section{APPENDICES}

This section contains fourteen appendices describing in detail various aspects of collective ion acceleration. Each is self-contained and, for the most part, has been submitted for publication in a scientific journal. Sec. II of the report sumrarizes the appendices, pointing cut significant features and tying the results together. 
[Reprinted from "Theoretical Division Annual Report, Ju1y 1975 - September 1976" (N. G. Cooper, ed.), LA-6816-PR, pP. 147-151.]

APPENDIX A

\title{
COMPUTER SIMULATION OF COLLECTIVE ION ACCELERATION
}

\author{
Brendon Godfrey and William R. Shanahan, T-6
}

For nearly two decades scientists have sought to utilize intense relativistic electron beams to accelerate significant ion currents to high energies.' Although only tentative progress has been made toward this goal, the apparent limitations of conventional accelerator technology have kept interest in a collective accelerator high. The inability of conventional technology to produce either stronger power suppilies or larger accelerating fields from them has meant the construction of ever longer and more costly devices to generate particles. On the other hand, the use of the collective fields of nonneutral electron beams, isolated from the accelerator walls holds the promise of much larger fields. Accelerator length could thus be reduced by factors of 50 and more. Moreover, the associated power supplies are well within the present pulsed-electron-beam technology. A collective ion accelerator, if it can be attained, would be much more compact, and consequently, less expensive than its conventional counterpart. The economy of such an accelerator would make it very attractive for production of both heavy and light ions in the medium-energy range $(\sim 100 \mathrm{MeV}-1 \mathrm{GeV})$. Availability of such devices could contribute in a major way to such areas as fundamental nuclear physics, superheavy nucleon synthesis, astrophysics, atomic and solid-state physics, ion-beam-initiated fusion, and radiation therapy.
Conceptually, one first obtains large-amplitude, low-velocity electrostatic wells within the electron beam. Potential wells can be created by the virtual cathode near the head of space-charge-limited beams, by large-amplitude Langmuir or cyclotron waves in the body of the beam, or, perhaps, by nonlinear solitary waves. One then loads ions into the slowly moving well and gradually accelerates it toward the beam velocity by appropriately varying one or more of the external parameter 3 affecting well velocity. Available quantities include background plasma density, applied magnetic field strength, drift tube radius, wall material, electron-beam energy, or beam current. The problem, of course, is actually to accelerate the well without causing it to break up or the ions to spill out.

Fecently, researchers have begun moving away from general considerations to fairly specific desigrs permitting experimental tests. One of the more extensively studied collective ion acceleration schemes is the autoresonant acceleration proposal of Austin Research Associates, Inc.' An intense relativistic electron beam propagates along a strong magnetic guide field in an evacuated cylindrical metallic waveguide as shown in Fig. III.9.1. Doppler-shifted slow cyclotron waves of low-phase velocity are excited in the beam, and ions trapped in them (see Fig. III.9.2). Acceleration is accomplished by suitably 


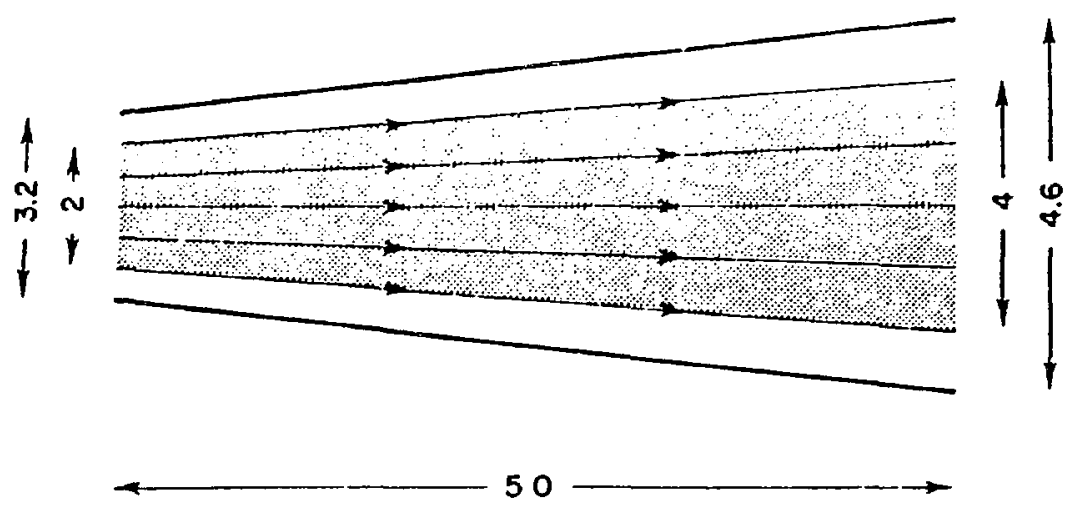

Fig. III.9.1.

Nominal experimental configuration corresponding to the simulations of Figs. III.9.2 and 3. Arrows show diverging magnetic field lines. The electron berım, occupying the shaded area, propagates from left to right. Dimensions are in $\mathrm{cm}$.

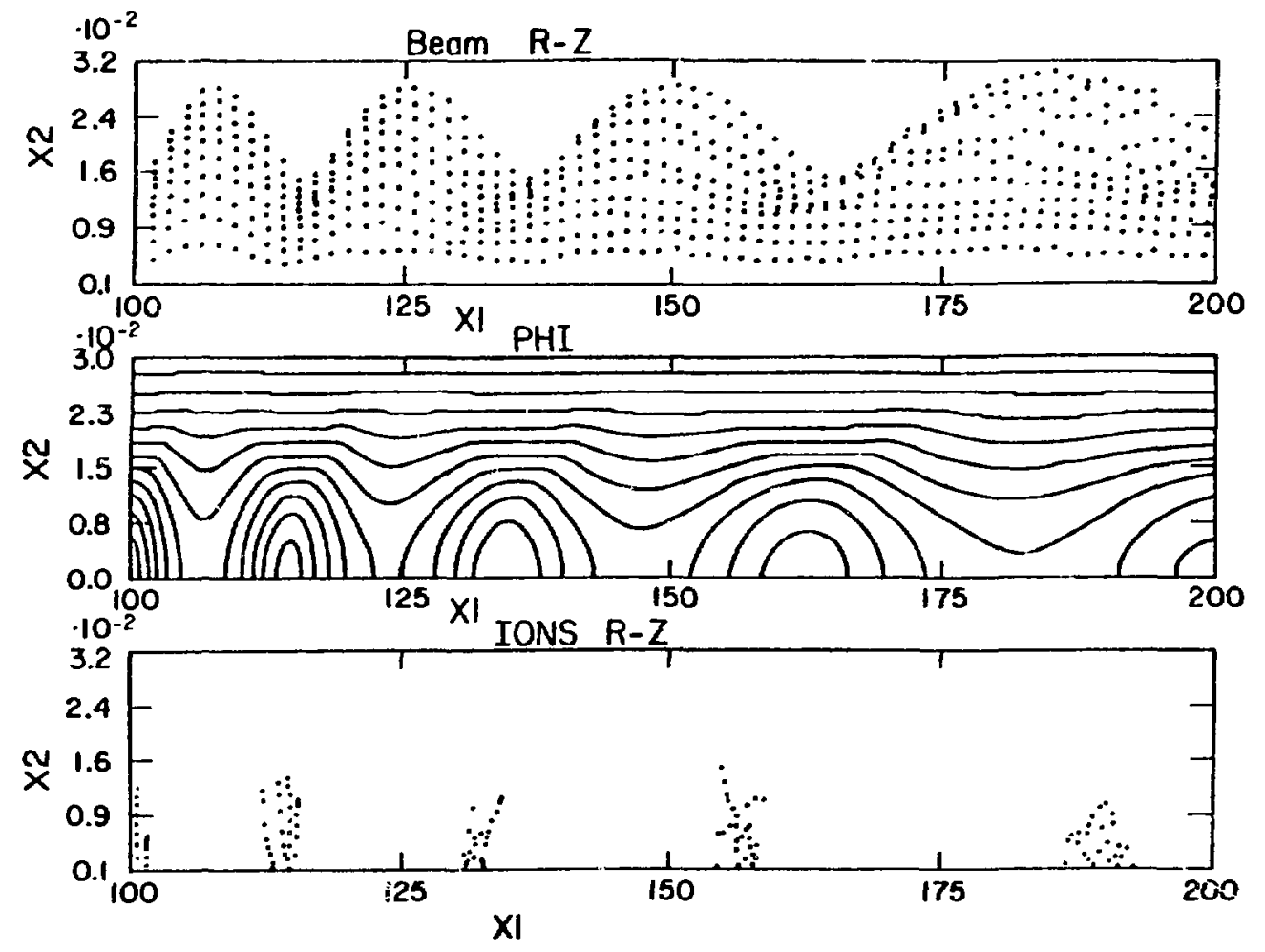

Fig. III.9.2.

Data from simulation of a cyclotron-wave-modulated electron beam with trapped ions propagating in a conical waveguide with open ends. Plots show (a) electron configuration space $(X 2-X 1),(b)$ electrostatic field equipotentials, and (c) ion configuration space (X2$X 1)$. Notation: $X 1=2, X 2=r / 2$. 
flaring the waveguide and applied magnetic field, so that the waves moving downstream gradually increase in wavelength and, hence, phase velocity.

Los Alamos Scientific Laboratory, Austin Research Associates, Air Force Weapons Laboratory, and others are collaborating in a feasibility experiment on autoresonant acceleration." Our task is to provide computational support, primarily large-scale computer simulations. Additionally, we are exploring alternative, less well-developed collective acceleration techniques in cooperation with Physics International Company and others.

Any useful numerical study of such acceleration schemes requires, at a minimum, a twodimensional, axially symmetric, relativistic, electromagnetic plasma simulation code. It must, in addition, be free of the numerical instabilities which have plagued earlier relativistic simulation codes." Since no such computer program existed, we began developing one in early 1975 . The code is now completely written and thoroughly tested. It treats selfconsistently the time-dependent trajectories of hundreds of thousands of individual beam electrons and ions over hundreds of plasma periods.

The code CCUBE constitutes state-of-the-art advances in two respects. First, it employs the recently derived partially Galerkin canonical momentum algorithm for advancing particle quantities.' This procedure eliminates completely the viruleni numerical Cherenkov instability and ameliorates other bothersome numerical problems. Secondly, the program is written for arbitrary separable, orthogonal coordinates. Thus, we can convert from, say, cylindrical to parabolic coordinates merely by redefining an array of metric elements. (Parabolic coordinates are, incidently, quite useful in some collective acceleration problems.) Nonuniform zoning of the spatial mesh is accomplished with identical ease.

A number of other features deserve mention. For compatibility with nonuniform zoning, we implemented variable particle weighting within particle types, with several distinct particle species permitted. The program supports highly flexible particle and field boundary conditions. Diagnostics include microfiche plots of particle and field information at various points in time, histories of selected quantities of interest, and color movies of key date. CCUBE is tightly optimized for efficient computer utilization.

Using this code, we first investigated injection, propagation, and virtual cathode formation for cold relativistic beams in evacuated cylindrical waveguides with magnetic guide fields of various strengths. Agreement with well-known analytic theory was good, so far as the theory went. In addition, we found that, for beam currents above the space-charge limit (the point at which self-electric fields reflect some beam particles), the beam tends to become hollow in radius. The virtual cathode (locus of points at which particles are reflected), moreover, was found to oscillate nonlinearly in size and position. For beams below the space-charge limit, shorting of the radial electric field by the anode foil at the waveguide entrance gave rise to large-amplitude zero-frequency (standing) cyclotron waves. Such waves are possibly quite detrimental to collective ion acceleration, interacting with the accelerating potential wells to cause ion loss. Several possible methods to suppress this effect have been suggested, and a few of these will be investigated computationally in the future. In addition, working with L. E. Thode of T-6, we are studying modifications of the phenomena just described by beam finite Larmour radius (effectively, thermal) effects.

As noted previously, autoresonant acceleration employs a gradually flaring waveguide and guide magnetic field. Simulations of propagation in this geometry produced no new effects for beams injected along the field lines. Of course, these twodimensional computations do not treat nonaxisymmetric behavior. To fill this gap, we considered analytically the possibility of rotational, sheardriven instabilities in three dimensions. The shear arises from longitudinal variation of the guide magnetic field. We found that, for a beam in the fast rotational mode (corresponding to cyclotron motion), a highly disruptive instability indeed exists. However, for the slow rotational mode (corresponding to E-cross-B drift about the beam axis), relevant to autoresonant acceleration, the instability is neg!igible.

With stable equilibrium beam propagation reasonably assured, we have performed preliminary simulations of ion acceleration." Figure III.9.2, a 
movie frame, presents characteristic results. The beam propagates left to right in a conical waveguide (mapped into a rectangle for plotting purposes). The guide field drops by a factor of 4 in strength. For these early trials, waves with ions already trapped were introduced ad hoc into the simulation. Actual wave growth will be studied in the near future. Figure III.9.3 gives the energy history of a typical ion clump, showing an energy increase by a factor of 8 through the acceleration region. Oscillations in the history are due to radial and longitudinal bouncing of ions in the potential well.

Although these few simulations, the first ever of traveling-wave collective ion acceleration, are generally quite encouraging, they exhibit one unexpected and somewhat worrisome feature. Oscillations of the various radial stream-lines gradually lose spatial coherence, giving rise to a systematic reduction of potential well depth. To better understand this effect, we have obtained numerically the complete electromagnetic linear dispersion relation for cylindrically symmetric electron beams with realistic radial profiles. Solutions show that, in sharp contrast to homogeneous plasma theory, the cyclotron spectrum consists of one discrete surface mode and a continuum of singular modes. The complete ramifications of this severe distortion of cyclotron waves by radial beam inhomogeneity are not yet fully resolved. Nonlinear and, perhaps, ther-

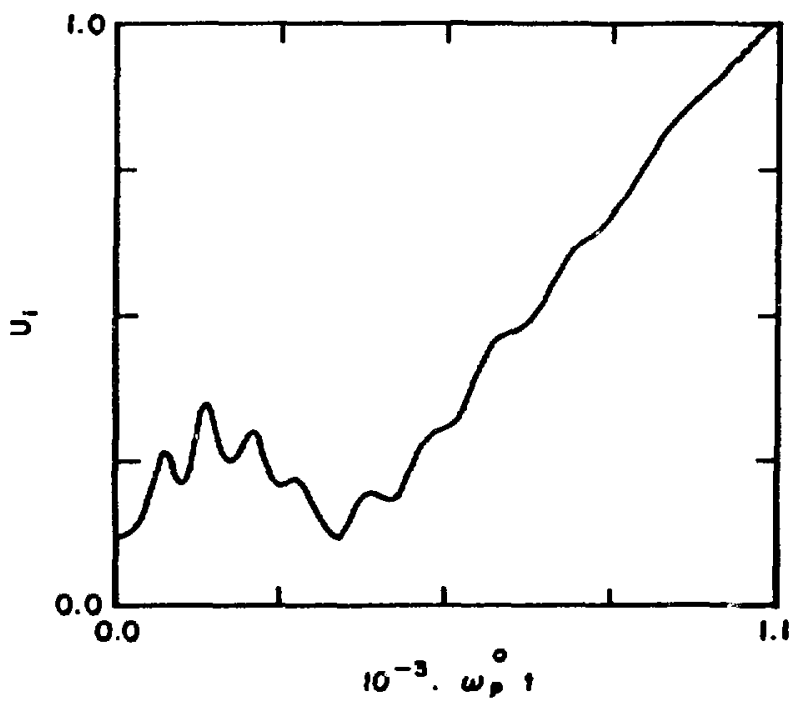

Fig. III.9.3.

Energy (arbitrary units) of accelerated ion bunch vs time. mal effects may be important. Incidentiy, the Langmuir wave spectra are much less affected by the naturally occurring radial inhomogeneity. Research is continuing.

We have rectist'y begun low-level investigations of two alternative acceleration proposals. In the first of these, the localized pinch model, ${ }^{\circ}$ the electron beam is radially confined not by a guide magnetic field but by a low-density ion background. Any local excess of ions causes the beam to pinch just downstream. Theory suggests that this ion clump and localized electron pinch can accelerate synchronously, drawing energy from the beam, provided the ion clump is stable. At present, we are simulating with CCUBE the response of electron beams to fixed ion clumps of different dimensions and densities. We have also developed a set of rough scaling laws.

The second alternative proposal entails controlling the motion of a virtual cathode in an electron beam flowing along a constant guide field in a flaring waveguide by varying the injected beam current or energy. Preliminary results suggest that virtual cathodes far removed from the anode foil are quite poorly behaved, exhibiting high noise levels and driving instabilities. However, this behavior is rich in difficult physics, and the final outcome of the investigation is still in doubt.

It should be emphasized that, in addition to the three proposals under investigation here, there are numerous other possible collection ion acceleracion techniques. Although these three proposals are among the better developed, it is not at all obvious that they will ultimately prove to be the most promisin ${ }_{\mathrm{b}}$. At present, collective ion acceleration is a wide-open field.

\section{REFERENCES}

1. A. A. Kolomensky, "Other Collective Methods," Proc. IX Intern. Conf. High Energy Accelerators (SLAC, Stanford, 1974), p. 254.

2. M. L. Sloan and W. E. Drummond, "Autoresonant Accelerator Concept," Phys. Rev. Lett. 31, 1234 (1973).

3. M. L. Sloan, Austin Research Assoc., private communication. 
4. B. B. Godfrey, "Canonical Momenta and Numerical Instabilities in Particle Codes, " J. Comp. Phys. 19 58-76 (1975).

5. B. B. Godfrey, "Numerical Simulation of Autoresonant Ion Acceleration," to be published in IEEE Trans. on Plasma Science.
6. S. D. Putnam, "Model of Energetic Ion Production by Intense Eiectron Beams, " Phys. Rev. Lett. 25, 1129 (1970). 
[To be published in IEEE Transactions on Plasma Science.]

APPENDIX B

NUMERICAL SIMULATION OF AUTORESONANT ION ACCELERATION *† by

Brendan B. Godfrey

\section{ABSTRACT}

Computational and analytic studies of the Autoresonant Acceleration proposal for collective ion acceleration are presented. Linear theory is reviewed, the electrostatic well depth is estimated nonlinearly, and an electron beam envelope equation is derived and solved. Two-dimensional numerical simulation results are given. Together, these caiculations demonstrate unneutralized electron beam equilibrium in a diverging magnetic gujde field, the behavior of large ampititude slow cyclotron waves in the beam, and the acceleration of test ions over short distances in the wave troughs. In addition, the computer simulations point up the need for improved understanding of the 1 inear theory of radially inhomogeneous noneutral beams and for methods of suppressing radial modulation at the diode-waveguide interface. 


\section{INTRODUCTION}

Over the past several years, many suggestions have been made for accelerating significant ion currents to high energies using localized space charge wells associated with non-neutral intense relativistic electron beams. Such potential wells can be created by the virtual cathode at the head of space charge limited beams, ${ }^{2-3}$ by large amplitude langmuir or cyclotron waves in the body of electron beams, ${ }^{4-7}$ or by nonlinear soliton structures. ${ }^{8-10}$ Conceptually, one loads ions into the low velocity weil and then gradually accelerates it toward the beam velocity by appropriately varying one or more of the external parameters affecting well velocity. Possible quantities include background plasma density, applied magnetic field strength, drift tube radius, wall material, beam energy, and beam current. The problem, of course, is actually to accelerate the well without causing it to break up or allowing the ions to spill out.

One of the more extensively studied collective ion acceleration schemes is the Autoresonant Acceleration proposal by Sloan and Drummond. $5,11-13$ An intense relativistic electron beam propagates along a strong magnetic guide field in an evacuated cylindrical metalic waveguide. Doppler-shifted slow (negative energy) cyclotron waves of low phase velocity are excited in the beam, and ions trapped in them. Acceleration is accomplished by suitably flaring the waveguide and applied magnetic field, so that the cyclotron waves moving downstream gradually increase in wavelength and, hence, phase velocity. Specifications for a feasibility experiment are presented in Ref. 14.

Many issues nust be resolved and difficulties overcome to brirg the Autoresonant Accelerator from a concept to a viable acceleration device. A suitable electron beam equilibrium needs to be obtained. Then a narrow band, large amplitude cyclotron wave must be produced, probably with a reactive or 
dissipative waveguide structure. This wave without ions must be able to propagate stably over several ineters. If it can, then a reasonable number of ions must be trapped and accelerated in the wave, the maximum ion current determined by various instabilities. In all these areas, one must contend with the voltage and current variations and with the finite Larmour radius effects which afflict real eiectron beams.

Here we address two questions, beam equilibrium formation and stable wave propagation, under relatively ideal conditions. Two-dimensional, relativistic, electromagnetic numerical simulations (in cylindrical and spherical coordinates) are employed together with simple nonlinear analytic models. Beam equilibrium is studied computationally by injecting a monoenergetic beam through a grounded entrance plane into an evacuated waveguide and magnetic guide field. The beam is seen to assume quickly an approximate rigid-rotor equillbrium in the slow rotational mode, the equilibrium parameters determined by the local values of the flaring waveguide, guide field and electron beam. Superimposed on the equilibrium are large standing waves excited by beam pinching near the injection plane (i.e., anode foil). A means will have to bs found to supress this undesirable mode.

To investigate wave propagation, we artificially create the equilibrium just mentioned, but without the standing wave, in a conical waveguide. Instead, travelling large amplitude cyclotron waves are impressed upon the beam ad hoc by modulating the beam radius and radial particle velocity according to solutions obtained from an analytic beam envelope equation and nonlinear well depth formula. We find that the wave accelerates as expected and is reasonably stable. However, it slowly decreases in amplitude, apparently due to phase mixing of radial stream lines. The phase mixing is associated with the beam's intrinsic radial kinetic energy variation. In spite of this 
difficulty, test ions trapped in the space-charge wells of these waves gain a factor of eight in energy.

Sec. II reviews the homogeneous beam linear theory of the cyclotron mode. The electrostatic well depth for large amplitude waves is determined in Sec. III. We derive a beam nonlinear envelope equation in Sec. IV and obtain analytic and numerical solutions. Simulation results are analyzed in Sec. V., and concluding comments presented in Sec. VI. 


\section{LINEAR THEORY}

Determination of the exact normal modes of an unneutralized relativistic beam confined in a waveguide, flared or not, is very difficult, and only limited progress have been made. ${ }^{10,15,16}$ However, for present purposes use of the homogeneous, unbounded cold plasma dispersion relation 5,17

$$
\begin{aligned}
& \left(\omega-k_{z} v\right)^{2}\left(\omega^{2}-\omega_{p}^{2} / \gamma-k^{2} c^{2}\right)\left[\left(\omega-k_{z} v\right)^{2}-\omega_{p}^{2} / \gamma^{3}\right]- \\
& \left(\omega_{c} / \gamma\right)^{2}\left(\omega^{2}-k^{2} c^{2}\right)\left[\left(\omega-k_{z} v\right)^{2}\left(\omega^{2}-k^{2} c^{2}\right)-\left(\omega_{p}^{2} / \gamma^{3}\right)\left(\omega^{2}-k_{z}^{2} c^{2}\right)\right]=0
\end{aligned}
$$

is of ten justified. Finite radius of the beam can then be taken into account by suitable quantization of $k_{\perp}$, while adiabatic variation of parameters along the beam is treated via the WKB approximation. In Eq. (1), $\omega_{p}$ and $\omega_{c}$ are the beam piasma and cyclotron frequencies, as usually defined nonrelativistically. Particle drift velocity is represented by $v$, with $\gamma=\left(1-v^{2} / c^{2}\right)^{-1 / 2}$. Components of the wave vector $k$ parallel and perpendicular to the beam flow are $k_{2}$ and $k_{1}$, respectively.

Eq. (1) is valid to the extend that the equilibrium self fields and rotation of the beam can be neglected, and that beam density and drift velocity are independent of radius. This is true for a rigid rotor equilibrium in the slow rotation mode when

$$
\begin{aligned}
& \nu / \gamma \ll 1 \\
& \omega_{p}^{2} / 2 \gamma^{2} \omega_{c} \ll c / a_{o}
\end{aligned}
$$


In Eq. (2), $v$ is Budker's normalized current parameters, $v=I \mathrm{e} / \mathrm{mc}^{3} .18$

Eq. (3) describes the beam anguiar velocity, with $a_{0}$ the equilibrium radius. When $\omega^{2} \ll k^{2} c^{2}$ and $\omega_{p}^{2} / \gamma^{3} \ll\left(\omega-k_{z} v\right)^{2}$, as will generally be true throughout the accelerator, Eq. (1) can be solved to yield for the slow cyclotron wave $\mathrm{e}^{12}$

$$
\omega=k_{z} v-\left(\omega_{c} / \gamma\right) k^{2} c^{2} /\left(k^{2} c^{2}+\omega_{p}^{2} / \gamma\right)
$$

For sufficiently slow variation of the parameters $\omega_{c}, \omega_{p}$, and $k_{1}$ along the accelerator (i.e., scale lengths large compared to $\lambda=2 \pi / \mathrm{k}_{z}$ ), Eq. (4) can be treated as locally correct in $z$. The wave phase velocity is, then, approximately $y^{5}$

$$
v_{w}=v \omega /\left(\omega+w_{c} / \gamma\right)
$$

W1th w chosen small, the phase velocity can be increased from near zero to a large fraction of the beam velocity merely by spatially decreasing the guide magnetic field strength. This is the essence of Autoresonant Acceleration.

We use conservation of energy flux along the beam ${ }^{5}$ (equivalently, WKB theory) to determine how the well depth $\Delta \phi$ associated with the slow cyclotron wave varies with position:

$$
\Delta \phi \propto\left(1+\alpha k_{z}^{2} a_{0}^{2}\right)^{-1}
$$


$\alpha$ is a constant of order $0.1-0.3$, depending on the details of the radial eigenmode structure. Except near the waveguide entrance, $\mathrm{k}_{\mathrm{z}} \mathrm{a}_{0}<1$. Thus, $\Delta \phi$ is essentially constant throughout most of the acceleration process.

If ions are not to slip out of the potential wells, well acceleration, $\dot{v}_{w^{\prime}}$, must never exceed the maximum ion acceleration imposed by the parallal electrostatic field, $k_{z} \Delta \phi$. (Ion thermal energy, if small compared to the we11 depth, can be ignored.) From Eq. (4) - (5), this constraint gives

$$
\frac{\partial \omega_{c}}{\partial z}<\frac{(q / m) i}{(q / m) e} \frac{\gamma\left(\omega+\omega_{c} / \gamma\right)^{4} c^{2}}{v_{(u)}^{3}} \Delta \phi
$$

Here, $(q / m)_{i, e}$ is the ion or electron charge to mass ratio. We measure

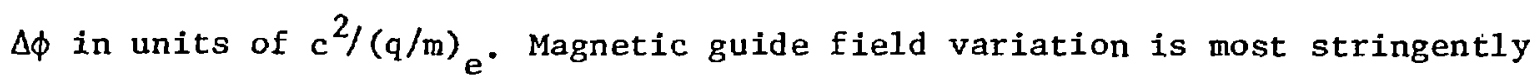
limited by (7) at the downstream end of the accelerator, where $\omega_{c} / \gamma$ approaches $\omega$. For $\Delta \phi$ constant, the magnetic field may decrease no faster than $\mathrm{z}^{-1 / 3} \cdot 11,14$ 
III. WELL DEPTH

Basically, the axisymnetric slow cyclotron wave with smallest perpendicular wave number is nothing more than a periodic modulation of the electron beam radius. We use this fact to estimate nonlinearly the electrostatic well depth of the wave.

In the long wavelength limit, $k_{2} a_{0} \ll 1$, the modulated beam can be described locally by the z-independent rigid rotor equilibrium, 15 provide again that (2) and (3) are satisfied. The electrostatic potential on axis is given by the well known space charge flow formula ${ }^{19,20}$

$$
v L=\phi\left[1-(\gamma-\phi)^{-2}\right]^{1 / 2}
$$

where $\mathrm{L}$ is a geometrical factor,

$$
\mathrm{L}=1+2 \ln (\mathrm{r} / \mathrm{a})
$$

$K$ is the waveguide radius. Eq. (8) and (9) have been verified both experimental1y ${ }^{21}$ and computationally. ${ }^{22}$ Plots of $v$ versus $\phi$ for various values of electron beam injection energy, $\gamma \mathrm{mc}^{2}$, are presented in Fig. 1. From curves such as those in Fig. 1, the cyclotron wave potential well depth $\Delta \phi$ can be determined as $\phi_{\max }-\phi_{\min }$, corresponding to $a_{\min }$ and $a_{\max }$ respectively, $\mathrm{In}_{\mathrm{Eq}} \cdot(8)$ and (9). To obtain $\Delta \phi$ as large as possible for a given beam energy, we should try to choose $\phi_{\text {min }}$ near zero and $\phi_{\max }$ near $\gamma-\gamma^{1 / 3}$, where $L v$ assumes its maximum value $\left(\gamma^{2 / 3}-1\right)^{3 / 2} \cdot 20$ However, $\phi_{\min } / \gamma$ near zero requires $V / \gamma$ quite small, since $L_{\text {min }} \geq 1$. In this case the beam modulation $a_{\max } / a_{\min }$ must be extremely large to provide 
a reasonably deep potentia1 we11, inviting unnecessary difficulties from beam thermal spread, angular rotation, and radial inhomogeneity. Also, small electron current limits the ion current which can be accelerated. Choosing $\phi_{\max }$ too near the flat portion of the curve may also prove dangerous. The beam is prone to strong longitudinal bunching, and perhaps break up, in this situation.

Therefore, it seems prudent, at least until nonlinear beam dynamics are better understood, to pick, $v / \gamma$ in the range $1 / 4-1 / 3$, In which case the maximum well depth is

$$
(\Delta \phi / \gamma) \sim(\nu / \gamma)
$$

Fig. 2, with $v / \gamma=0.25$ and $\gamma=7$, is a typical case. As $L$ ranges from 1 to $2, \phi / \gamma$ varies between 0.25 and 0.55 , giving $\Delta \phi / \gamma=0.3$. Beam modulation is $a_{\max } / a_{\min }=1.65$.

It is likely that $k_{z_{0}} a_{0} \ll 1$ will not be sarisfied at the beginning of the acceleration region. Finite wavelength effects can be treated Ith Green's function techniques, if laminar, paraxial beam flow 23 is assumed. We find that $\phi$ on axis is

$$
\begin{aligned}
\phi(z)= & 4 v \int d k_{z} \int d z^{\prime} e^{i k_{z}\left(z-z^{\prime}\right)}\left(k_{z} a\right)^{-1} \\
& {\left[I_{1}\left(k_{2} a\right) k_{0}\left(k_{z} a\right) / I_{0}\left(k_{z} a\right)+k_{1}\left(k_{z} a\right)-\left(k_{z} a\right)^{-1}\right] }
\end{aligned}
$$


where $a$ is a function of $z^{\prime}$. I and $k$ are modified Bessel functions of the first and second kinds. 24 For $k_{z} a<2$, Eq. (11) can be expanded in $k_{z}$ to yield

$$
\frac{\Delta \phi}{\gamma}=\frac{\nu}{\gamma}\left[\ln \frac{\left(\mathrm{a}^{2}\right)_{\max }}{\left(\mathrm{a}^{2}\right)_{\min }}-\frac{1}{4}\left|\frac{\mathrm{d}^{2}\left(\mathrm{a}^{2}\right)}{\mathrm{d} \mathrm{z}^{2}}\right|_{\max }\left(\ln \frac{\mathrm{R}^{2}}{\mathrm{a}_{0}^{2}}+\frac{3}{2}\right)\right]
$$

The first term in the brackets is an approximation to the infinite wavelength res 1 ts described above, the second term a finite wavelength correction which becomes significant for $k_{z} a_{0}$ of order unity.

Linearization of Eq. (12) gives

$$
\frac{\Delta \phi}{\gamma}=\frac{v}{\gamma} \frac{\Delta\left(a^{2}\right)}{a_{0}^{2}}\left[1-\frac{1}{4} k_{z}^{2} a_{0}^{2}\right]
$$

We have assumed $\ln \mathrm{R} / \mathrm{a}_{\mathrm{o}} \sim 1 / 4$, consistent with $\Delta L \sim 1$ and $\mathrm{R} \sim \mathrm{a}_{\max }$. Note that the functional form of Eq. (13) could have been anticipateci from Eq. (6). 


\section{ENVELOPE EQUATION}

A nonlinear envelope equation for zero frequency, axisymetric modulation of a ncnrelativistic beam in vacuum was obtained by Wang some years ago using the laminar, paraxial approximation. 25 Relativistic generalization of that result is straightforward, yielding

$$
\begin{aligned}
& v_{z}^{2} f^{1 / 2} \frac{d}{d z}\left[\left(1+\frac{v}{\gamma} \ln f\right) f^{-1 / 2} \frac{d}{d z} f\right]=\frac{\omega_{p}^{2}}{\gamma^{3}} /\left(1+\frac{v}{\gamma} \ln f\right)^{2} \\
& \quad+\left[\frac{\omega_{c}^{2}}{\gamma^{2}}\left(1-f^{2}\right)-2 \frac{\omega_{p}^{2}}{\gamma^{3}}\right] /\left[2 f\left(1+\frac{v}{\gamma} \ln f\right)\right] .
\end{aligned}
$$

Here, $f$ is the modulation, $f=a^{2} / a_{0}^{2}$. Eq. (14) is based on a rigid rotor equilibrium, 15 and is valid when (2) and (3) are satisfied.

Two approximate analytic solutions to Eq. (14) are roadily available. Linearizing ( $f \approx 1$ ), we find

$$
k_{z} v_{z}=\left[\left(\omega_{c}^{2} / \gamma^{2}\right)-\left(\omega_{p}^{2} / \gamma^{3}\right)(1-v / \gamma)\right]^{1 / 2},
$$

the upper hybred resonance modificd by beam rotation. In the low density limit, $\omega_{\mathrm{p}}^{2} / \gamma^{3} \ll \omega_{c}^{2} / \gamma^{2}$, we can derive the nonlinear result

$$
f=\left(A^{2}+1\right)^{1 / 2}+A \sin \left(\omega_{c}\left(z-z_{0}\right) / r_{z}\right)
$$


$A$ and $z_{0}$ are constants. Note that $f_{\max } \cdot f_{\text {min }}=1$. Eq. (16) could have been obtained more directly as the motion of a single particle in an external magnetic field.

Except for these two cases, it appears that Eq. (14) must be solved numerically. To do this, we first reduce the equation to quadrature,

$$
\begin{aligned}
& z-z_{0}=v_{z} \int \operatorname{df}\left(1+\frac{v}{\gamma} \ln f\right) \\
& {\left[2 \operatorname{cf}-\frac{\omega_{\mathrm{p}}^{2}}{\gamma^{2}}\left(1+f^{2}\right)+2 \frac{\omega_{\mathrm{p}}^{2}}{\gamma^{2}}\left(1+\ln \left(1+\frac{\nu}{\gamma} \ln f\right)\left(\frac{\nu}{\gamma}\right)^{-1} \mathrm{f}\right)\right]^{-1 / 2}}
\end{aligned}
$$

and then apply a standar' numerical integration formula. ${ }^{26}$ The constant C in Eq. (17) must exceed $\omega_{c}^{2} / \gamma^{2}-\omega_{p}^{2} / \gamma^{3}$.

Typical solutions are presenied in Fig. 3 and 4 . Fig. 3 contains plots of $\mathrm{f}$ vs. $\omega_{c} z / \gamma$ for $\mathrm{f}_{\min }=\mathrm{e}^{-1 / 3}, \mathrm{e}^{-2 / 3}, \mathrm{e}^{-3 / 3}$ and $\gamma^{1 / 2} \omega_{p} / \omega_{c}=0.2$, $v / \gamma=0.2$. Good agreement between the numerical results and Eq. (16) is apparent. Fig. 4 shows nonlinear wave number shifts as $\mathrm{f}_{\text {max }}$ and $\mathrm{f}_{\text {min }}$ vary, for $\nu / \gamma=0.2$ and $\gamma^{1 . / 2} \omega_{p} / \omega_{c}$. assuming values between 0.0 and 0.6 . In these Eigures the definitions $\omega_{c}^{R}=\omega_{c} / \gamma$ and $\omega_{b}^{R}=\omega_{p} / \gamma^{3 / 2}$ have been introduced for brevity. 
V. SIMURATIONS

We have carried out a series of PIC-CIC plasma computer simulations 27 providing preliminary insights into three aspects of autoresonant acceleration, zero frequency ripple of the electron beam surface, variation of cyclotron wavelength with radius, and acceleration of test ions in a conical waveguide. Computations were performed using CCUBE, a new two-dimensionai, relativistic, electromagnetic plasma simulation code. The code enploys improved algorithms which suppress certain numerical instabilities ${ }^{28}$ and is able to treat arbitrary separable, orthogonal coordinates. ${ }^{29}$ Numerical algorithms will be presented elsewhere.

The goal of these simulations is simply to demonstrate the acceleration of a few ions to modest energies under idealized conditions. Thus, the important questions connected with cyciotron wave growth, ion injection, magnetic field curvature, finite ion currents, ion extraction, and imperfections in the electron beam and guide magneic field are not addressed. A1so, no attempt is made to study parasitic instabilities except insofar as axisymmetric instabilities may occur over the short acceleration length employed. Simulations are all performed for a straight cone of length $100 \mathrm{c} / \omega_{\mathrm{p}}$. The cone doubles in radius from one end to the other, so that the applied magnetic field, diverging with the waveguide, decreases in strength by a factor of four. Because the beam electrons roughly follow the field lines, beam density falls by a factor of, again, four. Injection parameters for the beam are $\gamma=5$ and $\nu=1$. Thus, the initial beam radius is $2 \mathrm{c} / \omega_{p}$. Radius of the metal waveguide wall is chosen $1.3-1.6$ times this value for different simulations, corresponding to a cone half-angle of order $1^{\circ}-2^{\circ}$. 
In toore conventional terms, we are choosing $2 \mathrm{MeV}, 17 \mathrm{kA}$. electron beam. If its radius is $I \mathrm{~cm}$. at injection, then the waveguide is $50 \mathrm{~cm}$. long and the magnetic field $3.4 \mathrm{~kg}$ at the entrance plane. See Fig. 5. Time and length scales vary linearly with the beam injection radius.

From previous work, we understand reasonably well the propagation of a cold, low $v / Y$ electron beam in an evacuated cylindrical waveguide. 22 The first question to ask, then, is whether slow flaring of the waveguide changes propagation behavior. Therefore, we ground the injection plane, corresponding to an anode foil, and inject a cold beam free of scatter and modulation. The beam is in this way allowed to choose its own equilibrium. Fig. 6 is a movie frame from the first simulation, showing (a) electron positions in the waveguide, (b) contours of the electrostatic potential and (c) particle parallel momentum, $\gamma_{z} / c$, along the waveguide. (Note that the cone has baen mapped into a rectangle for convenience of plotting.) The behavior is as expected, with the beam adjusting adiabatically to the local guide field strength.

A significant feature in Fig. 6 is the large amplitude standing wave modulation of the beam radius. It arises due to a force imbalance at the anode foil, where $E_{r}$ vanishes but $B_{\Theta}$ does not. For weak modulation the amplitude is roughly

$$
f_{\max }-1 \nsucc 2 \gamma \omega_{p}^{2} / \omega_{c}^{2}
$$

but, as the right side of Eq. (18) approaches unity, $f_{\max }$ increases much more slowly. From Fig. 6, $f_{\max } ¥ 1.5$ wile $2 \gamma \omega_{p}^{2} / \omega_{c}^{2} \sim 2$. Such zero frequency cyclotron waves can also be excited by pinching in the diode, curved magnetic field lines, sharp density gradients in a low density background plasma, etc. They have been observed experimentally in a few instances. 30,31 
These standing waves are quite similar to the traveling waves with which we hope to accelerate ions, and for this reason they are particularly undesirable. The two waves, if of comparable amplitudes, beat spatially, spilling ions from the accelerating space charge wells. Additionally, the presence of two large amplitude waves greatly increases the possibilities for parametric instabilities. Several ways of suppresing the zero frequency modulation triggered by the anode foil come to mind, including employing larger magnetic fields at the diode, using a foilless diode, choosing a clever cathode design, setting a magnetic lense at the anode, ${ }^{14}$ and appropriately placing additional. foils downstream. 32 Each of these approaches entails certain problems, however, and no clean solution has yet been demonstrated. Incidently, increasing beam thermal spread is not acceptable, since this affects equally the desired and the untesired modes.

Eq. (2) has been invoked throughout Sec. II, III, and IV so that the variation in $\gamma$ with radius due to space charge can be ignored. Such a variation in $\gamma$ gives rise ostensibly to a variation of cyclotron mode parallel wavelength with radius for fixed $\omega$ and $\omega_{c}$ from Eq. (4). In the plot of $\gamma v_{z} / c$ in Fig. 6, we can see that $\gamma$ on average ranges between 3.5 and 4.5, implying $\Delta \mathrm{k}_{z} / \mathrm{k}_{\mathrm{z}} \sim 0.25$. Indeed, the upper plot in Fig. (6) indicates a total phase shift of roughly $\pi / 2$ between stream lines near the beam surface and those near the axis in the rightmost portion of the waveguide. Interestingly, if the well depth $\Delta \phi / \gamma$ of the accelerating cyclotron wave is comparable to $v / \gamma$, as argued in Sec. III, then this phase shift and the acceleration of ions scale together, because $\Delta \gamma / \gamma \downarrow v / \gamma$ across the beam. We then find, according to Eq. (4), that a radial phase shift of $\pi$ will occur spatially before singly charged ions can increase in energy by more than $2 \pi \gamma \mathrm{m}_{\mathrm{e}} \mathrm{c}^{2}$. In these terms the variation of $\gamma$ with radial position can never be ignored globally. 
There are at least two possible ill effects of the ralative phase shifts. Jus of phase coherence across the beam causes a reduction in we11 depth, $\Delta \phi$. Shifts between adjacent stream lines lead to stream line crossing and, perhaps, local turbulence. These problems should not be overstated, however, because we are applying homogeneous, linear thury to an intrinsically inhomogeneous, nonlinear situation. Additional investigation is required.

Having verified that a suitable equilibrium exists in the conical waveguide under consideration, we may turn out attention to simulating the acceleration of test ions. If we choose $\omega=0.023 \omega_{\mathrm{p}}^{0}$, then initially $k_{z}=0.46 \mathrm{c} / \omega_{p}^{0}$ from Eq. (4) for $\gamma \sim 4.5$ at the beam surface. At the waveguide exit $k_{z}$ drops to $0.14 \mathrm{c} / \omega_{p}^{0}$ for an increase in phase velocity from $0.05 \mathrm{c}$ to $0.16 \mathrm{c}$. (By $\omega_{\mathrm{p}}^{\mathrm{o}}$ we mean the value of $\omega_{p}$ at the waveguide entrance.) Thus, an ion traveling at constant phase relative to a given wave could gain a factor of ten in energy. However, as discussed in Sec. II, the magnetic field profile $\omega_{c} \sim z^{-2}$ corresponding to our conical waveguide decreases too rapidly, so that ions slip in phase relative to the waves and eventually become untrapped. In order that ions do not slip out of the well until it just reaches the far end of the waveguide, we set $\Delta \phi \sim 1$ and $(q / \mathrm{m})_{i}=500$. The mass ratio, although unphysical, is sufficiently large that the ion and electron time scales are well separated, and results can easily be scaled to real ion masses.

To avoid the zero frequency modilation described above, we set $\partial \phi / \partial z=0$ instead of $\phi=0$ at the entrance plane of the simulation. Particles are injected with $\gamma=\gamma_{0}-\phi\left(\gamma_{0}=5\right.$, as before $)$ and with enough angular velocity to provide approximate racial force balance. A beam launched this way adjusts quickly to its true equilibrium with almost no unwanted modulation. Because we are putting aside for now the issue of 
cyclotron wave growth, we create a large amplitude wave ad hoc at the entrance plane by superimposing a modulation of the electron radial position and velocity according to Eq. (16), doppler shifted to give the desired frequency. An amplitude $A=0.6$ is appropriate to $\Delta \phi \sim 1$. Ions are injected in ciumps already trapped at the bottom of the space charge wells and moving with a velocity $0.05 \mathrm{c}$. Ion density is 1 ocally $10^{-3}$ the electron density and, when averaged over the beam volume near the waveguide entrance, is lower still by a factor of four. Influence of the ions on electron beam dynamics is, therefore, negligible.

Fig. 7 is a movie frame from tilis simulation at time $t=1100 / \omega_{p}^{\circ}$. It differs in format from Fig. 6 only in that the bottom plot is ion configuration space. We see that the ions remain bunched as they propagate, but the clumps slowly drop behing their respective accelerating wells, as expected. The rightmost clump has shifted in phase by $-\pi / 2$ and is about to slip from its well. An energy history of this clump is presented in Fig. 8. Energy gair of a factor of eight is consistent with the factor of ten predicted above, if account is taken of the relative phase shift. Oscillations in the energy are due to bouncing of the ions in the electrostatic well, 12 the fast oscillations corresponding to radial motion and the slow oscillations to longitudinal motion.

Variation of longitudinal wavelength with radius is evident in Fig. 7 , but is not so pronounced as in the earlier simulation, Fig. 6. Whether the reduction is due to nonlinear effects, details of the wave injection, or some other cause is unclear. The change in well depth stemming from the phase shifts among stream lines is partially masked by the slow spatial decrease in $\phi$ associated with a slight expansion of the electron beam relative to the flared waveguide wall. 
VI. CONCIUSIONS

We have presented analytic and simulational results basic to autoresonant ion acceleration. The linear theory of cyclotron waves in a homogeneous relativistic beam has been reviewed. In the long wavelength limit, the wave nonlinear electrostatic well depth and the beam nonlinear envelope equation have been derived. Finally the autoresonant acceleration of lons has been self-consistenly simulated for the first time. We concluade that such acceleration is indeed possible, at least for the ideal conditions assumed here. Also, we have tentatively decided that $k_{z^{a}} \leq 1, \Delta \phi \eta v$, and $v / Y \sim \frac{1}{4}-\frac{1}{3}$ are the most suitable acceleration conditions.

The simulations have raised but not resolved the questions of large amplitude standing beam modulation and of radial variation in the cyclotron wavelength. Beam pinching and modulation at the waveguide entrance would not seem to be an intrinsic problem, and probably can be overcome by one or more of the methods 1isted in the preceding Section. Sloan, Uglum, and others are pursuing this issue. 14

The apparent radial dependence of the cyclotron longitudinal wavelength is, of course, only one aspect of the problems associated with linear theory in an inhomogeneous plasma. Based on the few published investigations into this area, ${ }^{11,33-34}$ we speculate that the complete cold beam cyclotron spectrum corisists of a few discrete undamped modes corresponding to quantized values of $k_{1}$ plus a continuum of sjigular modes localized in radius and decaying as some small inverse power of $z$. To verify this guess, we have linearized the cold fluid equations about realistic relativistic electron beam equilibria and have begun solving them together with Maxwell's equations numerically and, when possible, analytically. This exercise should also provide interesting information on the stability of non-rigid rotor equilibria ${ }^{35}$ and on the structure of normal modes for beams near the top of the curves in Fig. 1. 36 
Final1y, we wish to remphasize one deficiency of the numerical simulations. They are axisjmmetric. At a later date we will perform simulations in $r-\theta$ geometry to investigate kink, 12 diocotron, 15 and other angle dependent instabilities. Analytically, we have already skown that the axial shear in beam rotation frequency due to the varying magnetic guide field drives instabilities only for non-neutral beams in the fast mode of rotation. 37 


\section{ACKNOWLEDGMENTS}

We are endebted to R. J. Faehl, R. B. Miller, W. R. Shanahan, M. L. Sloan, D. C. Straw, L. E. Thode and others for their valuable suggestions and criticisms. 
* supported jointly by the Air Force office of Scientific Research and the Energy Research and Development Administration.

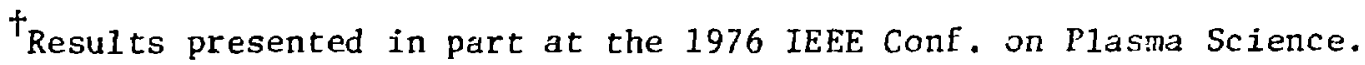

1. For a recent review, see A. A. Kolomensky, Proc. IX Int. Conf. High Energy Accelerators (SLAC, Stanford, 1974), p. 254.

2. C. L. Olson, Part. Acce1. 6, 107 (1975); C. L. 01son, Proc. Conf. Electron Beam Research and Technology (Sandia Laboratories, Albuquerque, 1975), Vol. 2, p. 312 .

3. R. B. Miller, AFWL-DYS-TN-75-115 (Air Force Weapons Laboratory, Albuquerque, 1975); R. J. Faehl, R. B. Miller, and B. B. Godfrey, Bu11. Am. Phys. Soc. 21, 1165 (1976).

4. V. I. Veksler, Atom. Ener. 2, 525 (1957); J. M. Wachtel and B. J. Eastland, Bu11. An. Phys. Soc. 14, 1047 (1969).

5. M. L. Sloan and W. E. Drummond, Phys, Rev. Lett. 31, 1234 (1973).

6. P. Sprangle, A. T. Drobot, and W. N. Manheiner, Phys. Rev. Lett. 36, $1180(1976)$

7. S. V. Yadavalli, App1. Phys. Lett. 29, 272 (1976).

8. S. D. Putnam, Phys. Rev. Lett. 25, 1129 (1970); S. J. Putnam, IEEE Nuc. Sc1. 18, 496 (1971).

9. V. N. Tsytovich, Preprint. 
10. B. B. Godfrey, Bu11. Am. Phys. Soc. 21, 1183 (1976).

11. W. E. Drumiond, G. I. Bourianoff, D. E. Hasti, W. W. Rienstra, M. L. Sloan, and J. R. Thompson, AFWL-TR-74-343 (Air Force Weapons Laboratory, Albuquerque, 1975).

12. W. E. Drummond, G. I. Bourtanoff, E. P. Cornet, D. E. Hasti, W. W. Rienstr M. I. Sloan, H. V. Wong, J. R. Thompson, and J. R. Uglum, AFUL-TR-75-296 (Air Force Weapons Laboratory, Albuquerque, 1976).

13. W. E. Drummond, G. I. Bourianoff, E. P. Cornet, D. E. Hasti, W. W. Rienst] M. L. Sloan, J. R. Thompson, J. R. Iglum, and H. V. Wong, AFWL-TR-76-152 (Air Force Weapons Laboratory, Albuquerque, 1977).

14. W. E. Drummond, G. I. Bourianoff, D. F. Brower, K. W. Gentle,

D. E. Hasti, W. W. Rienstra, M. L. Sloan, J. R. Thompson, J. R. Uglum, P. Wildi, and H. V. Wong, unpublished.

15. R. C. Davidson, Theory of Nonneutral Plasmas (Benjamin, Reading, 19?4).

16. M. I. Sloan, Bul1. Am. Phys. Soc. 21, 1147 (1976).

17. B. B. Godfrey, W. R. Shanahan, and L. E. Thode, Phys. Fluids 18, 346 (1975

18. G. I. Budker, Atom. Ener. 1, 9 (1956).

19. L. S. Bogdankevich and A. A. Rukhadze, Sov. Phys. Uspekhi 14, 163 (1971).

20. B. N. Breizman and D. D. Ryutov, Nuc. Fus. 14, 873 (1974).

21. R. B. Miller and D. C. Straw, submitted to J. Appl. Phys. 
22. B. B. Godfrey and L. E. Thode, Bull. Am. Phys. Soc. 20, 1271. (1975).

23. P. T. Kirstein, G. S. Kino, and W. E. Waters, Space-Charge Flow (McGraw-Hill, New York, 1967), Ch. III-IV.

24. M. Abramowitz and I. A. Stegun, Handbook of Mathematical Functions (National Bureau of Standards, Washington, 1964); Ch. 9.

25. C. C. Wang, Proc. IRE 38, 135 (1950).

26. Ref. 24, Ch. 25, formula 25.4 .39$, p. 889 .

27. R. L. Morse, Meth. Comp. Phys. 9, 213 (1970); C. K. Birdsall and D. Fuss, J. Comp. Phys. 3, 494 (1969).

28. B. B. Godfrey, J. Comp. Phys. 19, 58 (1975).

29. P. M. Morse and H. Feshbach, Methods of Theoretical Physics (McGrawHi11, New York, 1953), Vol. I, Ch. 5.1.

30. R. A. Mahaffey, S. A. Goldstein, R. C. Davidson, and A. W. Trivelpiece, Phys. Rev. Lett. 35, 1439 (1975).

31. R. B. Miller and D. C. Straw, private communication.

32. T. Goodlove, private communication.

33. E. A. Frieman, M. L. Goldberger, K. M. Watson, S. Weinberg, and N. M. Rosenbluth, Phys. Fluids 5, 1976 (1962).

34. Z. Sedlacek, J. Plas. Phys. 5, 239 (1971); 6, 187 (1971). 
35. Stability ố nonrelativistic rigid rotor equilibrium is analyzed

in ‥ v. Wong, M. L. Sloan, J. R. Thompson, and A. T. Drobot, Phys. Fluids 16, 902 (1973). See also Ref. 15, pp. 155-159.

36. B. B. Godfrey, submitted to IEEE Trans. Plas. Sci. 


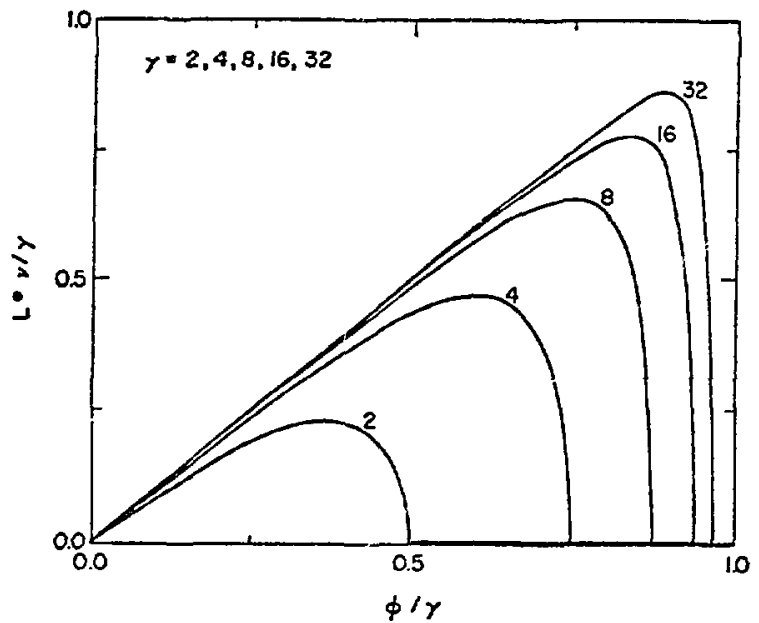

Fig. 1. Nozmaliced current $v / \gamma$ vs. normalized potential well depth $\phi / \gamma$ for the cylindrically symmetric equilibrium of a nonneutral beam in a strong magnetic guide field with injected particle energies $\gamma=2,4,8,16,32$.

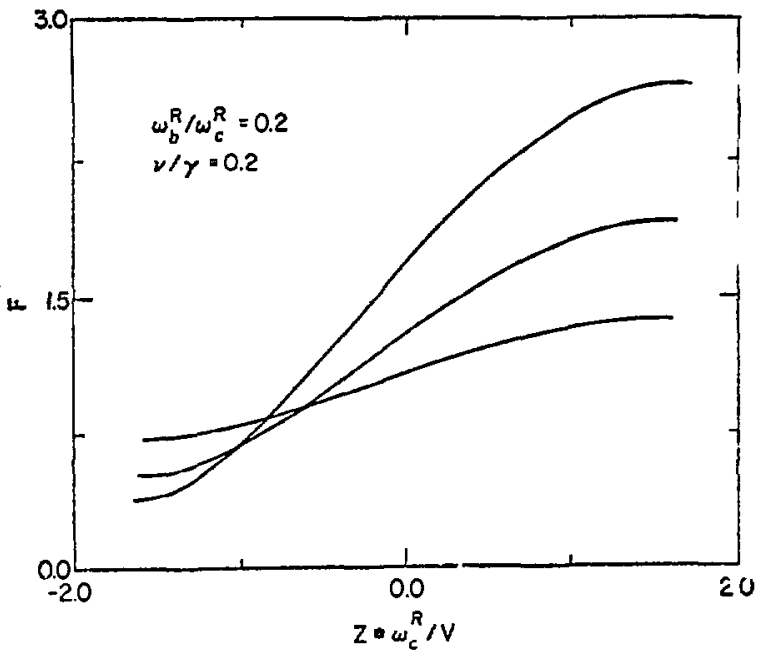

Fig. 3. Solutions of $\mathrm{Eg}$. (17) for $\mathrm{En}\left(\mathrm{f}_{\mathrm{min}}\right)=-I / 3$, $-2 / 3,-1$ and $\omega_{b}^{R} / \omega_{c}^{R}=0.2, \nu / \gamma=0.2$.

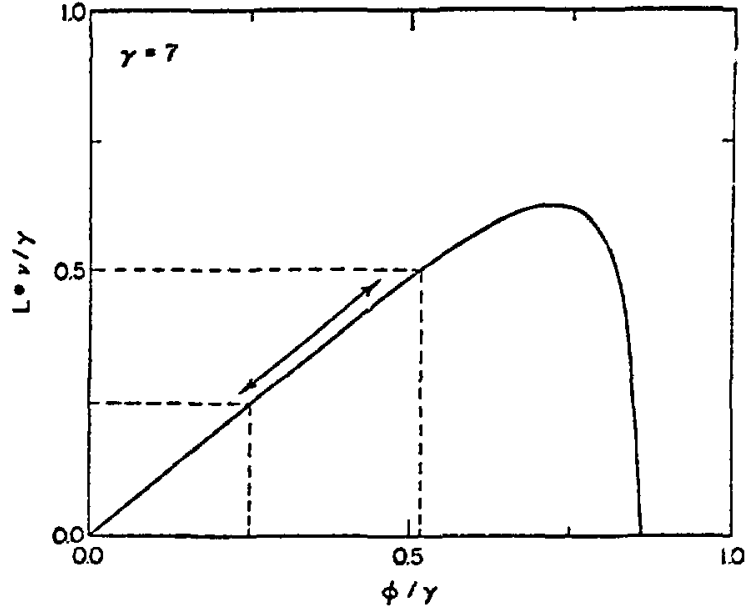

Fig. 2. Variacion of potential well depth with beam radius for a $\gamma:=7$ beam with long wavelength radial moriulation.

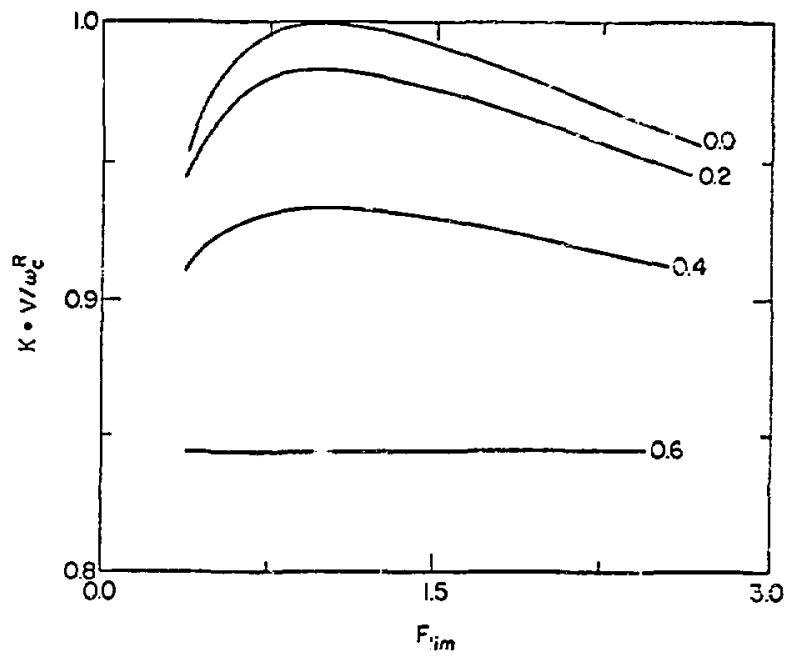

Fig. 4. Modulation wavenumber from Eq. (1i) vs. extrema of $f$ with $v / \gamma=0.2$ and $\omega_{b}^{R} / \omega_{c}^{R}=0.0,0.2,0.4,0.6$. 


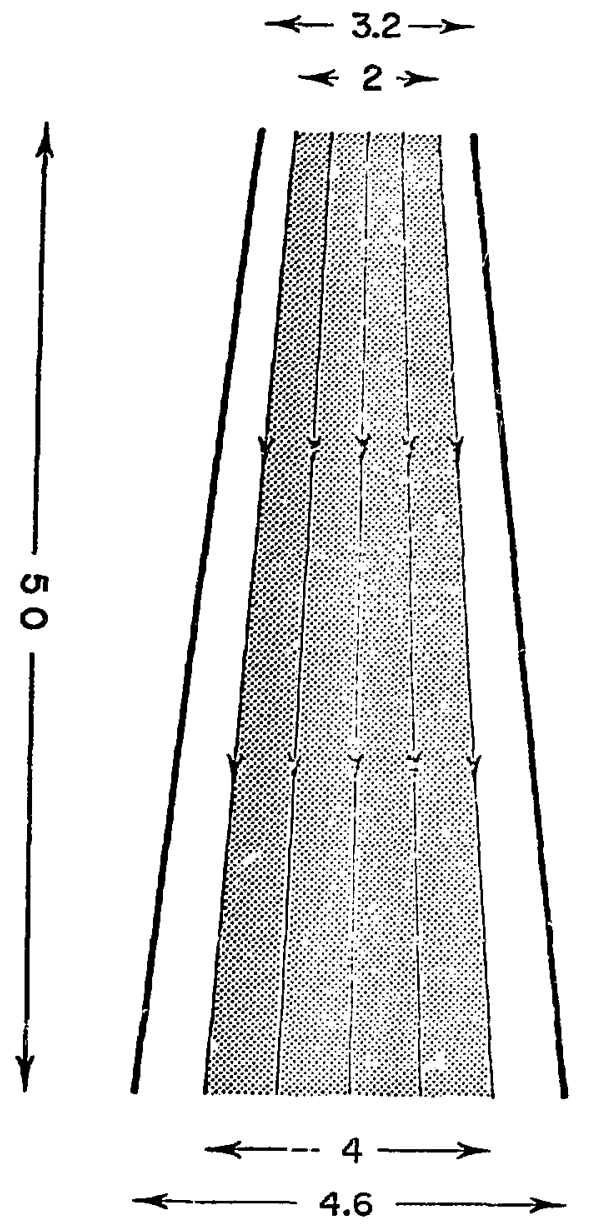

Fig. 5. Nominal experimental configuration corresponding to the simulations on Figs. 6 and 7. Arrows show diverging magnetic fteld lines. The electron beam, occupying the shaded area, propagates from left to right. Dimensions are in $\mathrm{cm}$.
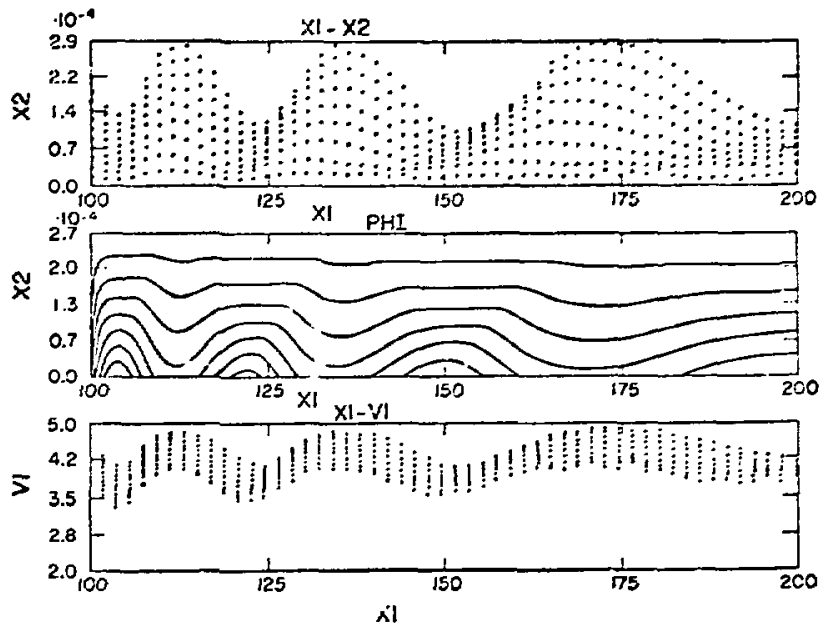

Fig. 6. Data from simulation of $v=1, y=5$, $\omega_{c}^{O} / \omega_{0}^{O}=2$ electron beam propagating in a conical waveguide with grounded entrance plane (at left). Plots show (a) electron configuration space $(\times 2-\times 1)$, (b) electrostatic fleld equipotentials, and (c) electron phase space (VI-XI). Notation: $X 1=$ $z, x 2=1 / 2\left(r^{2} / z^{2}\right), v I=\gamma v_{z} / c$.
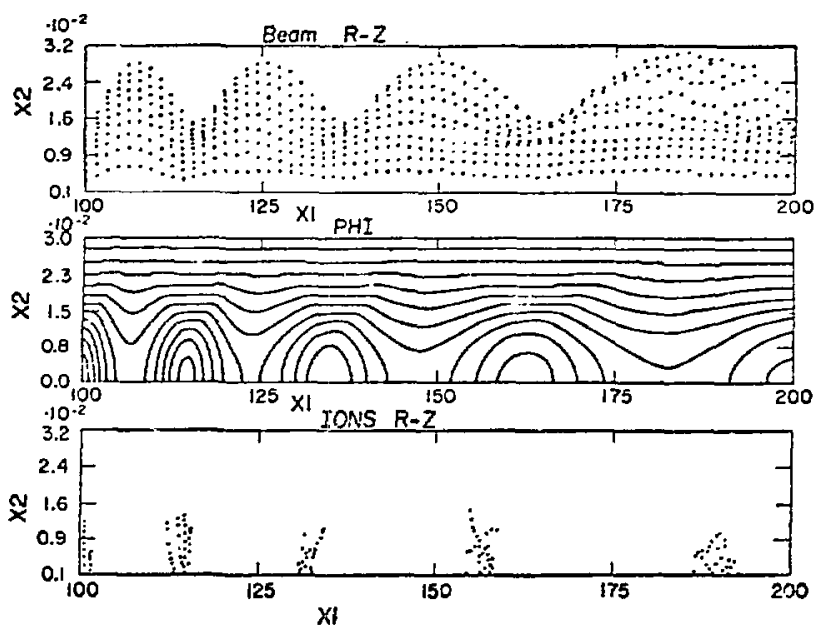

Fig. 7. Data from simulation of $v=1, \gamma=5$, $\omega_{c}^{O} / \omega_{p}^{O}=2$ modulated electron beam with trapped ions propagating in a conical waveguide with open ends. Plots show (a) electron configuration space (X2-X1), (b) electrostatic field equipotentials, and (c) ion configuration space $(\mathrm{X} 2-\mathrm{X} 1)$. Notation: $\mathrm{X} 1=\mathrm{z}, \mathrm{X} 2=\mathrm{r} / \mathrm{z}$. 


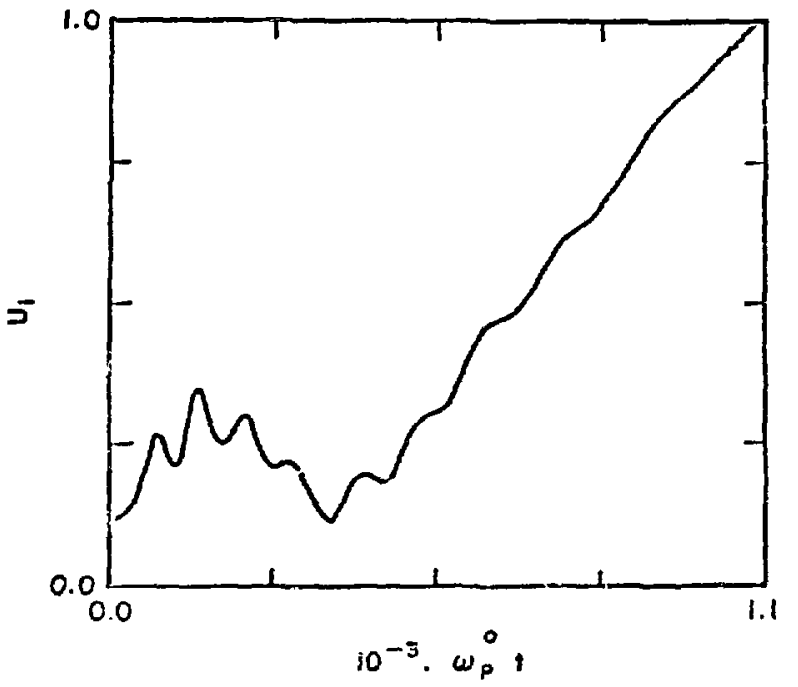

Fig. 8. Energy (arbitrary urits) of accelerated ion bunch vs. time. 
['io be submitted for publication in revised form. ]

APPENDIX C

VACUUM PROPAGATION OF A RELATIVISTIC ELECTRON BEAM: EQUILIBRIUM

by

Lester E. Thode

Brendan B. Godfrey

William R. Shanahan 


\section{INTRDDUCTION}

$\Lambda$ number of applications utilizing an intense relativistic electron beam require that the beam be injected into an evacuated cylindrical drift tube. In the absence of an externally imposed axial magnetic field, the self-electric field of the beam always exceeds the selfmagnetic field, and thus the beani simply expands until it collides with the drift tube wall. If an external magnetic field ${ }_{0}$ is imposed at the source, radial force balance can occur after the beam has expanded sufficiently. However, for such an equilibrium configuration, the beam may be subject to instability or to the formation of a virtual cathode. For collective ion acceleration produced by coherent wave formation, ${ }^{1-3}$ microwave generation, ${ }^{4-7}$ autoacceleration, ${ }^{8}$ inertially confined plasma heating, 9,10 and radial pulse line accelerator 11,12 injection, a knowledge of achievable self-consistent, propagating laminar flow equilibria is desirable. This implies stability and the existence of a coherence length over which the laminar flow is preserved. In contrast, collective ion acceleration generated by control of macroscopic beam fields ${ }^{13-16}$ (neutral gas breakdown) and optimization of ion current in reflex $\operatorname{triodes}^{17}$ depends upon understanding the development and time dependence of a virtual cathode.

For the most part, theoretical investigations ${ }^{18-29}$ of equilibrium, stability, and space charge limiting current have assumed at least one of the following: i) the initial beam distribution is a Dirac delta function, $i i)$ the equilibrium is axially independent and a rigid rotor, or 11i) the externally imposed magnetic field is essentially infinite. Generally speaking, at least one, if not all three, of these assumptions 
are violated experimentally. ${ }^{30-36}$ Thus, in the present paper we attempt to bridge the gap between theory and experiment, and obtain some insight into finite magnetic field strength requirements and effects of anode foll scattering.

Ir Sec. II a brief review of analytical work associated with the determination of the space charge limiting current is given. This includes the recent iteration results of Thompson and Sloan, 21 and Genoni and Proctor 22 for the iimiting current of a solid beam, which take into account the radial inhomogeneity of the beam parameters but assume an infinite axial guide field.

A discussion of two codes is given in Sec. III. Firstly, a new generation particle code CCUBE, which obtains two-dimensional, time dependent solutions of beam propagation in any orthogonal, separable coordinate system, is discussed. The code contains numerical equivalents of experimental diagnostics such as Faraday cups, calorimeters, diamagnetic loops, field and current probes, and Rogowski loops. A second code GRADR obtains the self-consistent equilibrium and space charge limiting current of a radially inhomogeneous, cylindrically symmetric, cold fluid beam in a finite strength external magnetic field. It then computes linear eigenmodes for that equilibrium.

An examination of equilibrium, stability, and space charge limiting current for finite magnetic field strength, but in the absence of foil scattering, is carried out in Sec. IV. Defining $\omega_{c}=e B_{0} / \mathrm{mc}$ as the cyclotron frequency and $\omega_{b}^{0}=\left(4 \pi n_{b}^{0} e^{2} / m\right)^{1 / 2}$ as the beam plasma frequency at the source, we find that at a fintte magnetic fleld strength of

$$
w_{c} \geq 5 w_{b}^{0}
$$


is rcquired for the infinite magnetic field limiting current calculations to be valid. For a magnetic field strength below this level, the spacecharge limiting current increases somewhat. Preliminary investigations show no evidence of significant instability in solid beam equilibria. For injection current exceeding the space charge limiting current, the position and strength of the virtual cathode are found to oscillate in time. Under these conditions, the average transmitted current can exceed the static limit by approximately $10 \%$.

Anode foil scattering is considered in Sec. V. For a beam with a relativistic factor of $\gamma_{0}$ at the source, the effect of anode foil scattering on the space charge limiting curtent is negligible if

$$
\gamma_{0}^{4 / 3}\left(\gamma_{0}^{2 / 3}-1.0\right)>100 \mathrm{~F}
$$

where $F$ is a foil scattering function depending only on foil parameters. An expression for the limiting current as a function of the scattering angle is derived, and is in good agreement with simulation and experiment.

Finally, in Sec. VI we compare our analysis and simulation with a number of experimental results. Overall the agreement is good, although some measurements, such as diamagnetic loops, are found to be of marginal utility. 
We assume the bean source is immersed in an externally inposed axial magnetic field $\mathrm{B}_{0}$. A discussion of the magnetically shielded source is given by Reiser. ${ }^{29}$ Furthermore, we assume that the magnitude of $B_{0}$ is sufficient to provide radial force balance with a negiigible change in the beam radius $a_{0} \cdot$ Azimuthal symmetry implies conservation of caronical angular momentum. It follows that azimuthal $v_{\theta}$ and radial $v_{r}$ velocity components are also negligible. At the source the axial current density

$$
J_{z}(r)=-\left[U(r)-U\left(r-a_{0}\right)\right] \text { e } n_{b}^{0} v_{z}^{0}
$$

and electron kinetic energy

$$
E(r)=\left[U(r)-U\left(r-a_{0}\right)\right]\left(\gamma_{0}-1.0\right) m c^{2}
$$

are constant. The electron charge and mass are $-e$ and $m$, and $U$ is the unit step function. These assumptions imply that the local beam distribution is a Dirac delta function

$$
f_{b}^{0}=\delta\left(p_{1}-p_{0}\right) \delta\left(p_{1}^{2}\right) / 2 \pi
$$

With appropriate boundary conditions, the solution of

$$
\frac{1}{r} \frac{\partial}{\partial r}\left(r \frac{\partial \gamma}{\partial r}\right)+\frac{\partial^{2} \gamma}{\partial z^{2}}= \begin{cases}-\left(4 \Delta_{0} / a_{0}^{2}\right) \frac{\gamma}{\left(\gamma^{2}-1.0\right)^{1 / 2}} & \text { for } r \leqslant a_{0} \\ 0 & \text { for } r>a_{0}\end{cases}
$$


yields the steady state electron energy $\mathrm{Ymc}^{2}$ within an evacuated cylinder of radius $R$ and length $I$. We have defined

$$
\Delta_{0} \equiv \pi a_{0}^{2} n_{b}^{0} e^{2} v_{z}^{0} / m c^{3}=I_{0} /\left(m c^{3} / e\right)
$$

as the dimensionless source current, where $\mathrm{mc}^{3} / \mathrm{e}=17 \mathrm{kA}$. The limiting current $\Delta_{L}\left(\omega_{c} / \omega_{b}{ }^{0}, \theta_{s}\right)$ is an implicit function of $\gamma_{0}$ and $R / a_{0}$, but is an explicit function of both the external magnetic field strength $\omega_{c} / \omega_{b}^{0}$ and the anode foil scattering $\theta_{S^{*}}$ In general, we define

$$
\Delta_{L} \equiv \Delta_{L}(\infty, 0.0)
$$

as the infinite magnetic field, cold beam limit.

The singularity at $\gamma=1$ in (la) is associated with the possible formation of a virtual cathode. If $R \gg L$, expressior (1) reduces to the solution of a one-dimensional planer diode. In this approximation, Voronin et al. ${ }^{27}$ find that a virtual cathode can form when $\Delta_{\theta}=\Delta_{L}$, where the limiting current $\Delta_{L}$ is a function of $\gamma_{0}$ and L. For $\Delta_{0}<\Delta_{L}$, the existence of two equilibria is predicted. In contrast, for an injection current above the limiting current, the formation o the virtual cathode uccurs increasingly closer to the anode and the transmitted current $\Delta_{\mathrm{T}}$ decreases below $\Lambda_{\mathrm{L}}$ as $\Delta_{0} / \Delta_{\mathrm{L}}$ increases. As discussed by Birdsal1 and Bridges, ${ }^{24}$ Dunn and Ho, ${ }^{25}$ de Jagher et al., ${ }^{26}$ and Poukey and Rostoker, 28 the actual physical solution is one in which the position and well depth of the virtual cathode oscillate in time. Calculations indicate that the frequency of oscillation is near. the beam piacma frequency, and tends to increase as the ratio $\Delta_{0} / \Delta_{L}$ increases.

The typical experimental configuration is $L \gg R$. In this regime, Miller and straw ${ }^{35}$ provide a criterion for neglecting the presence of endplates, 


$$
L / R \geq 2.58\left(a_{0} / R\right)^{0.133}
$$

If expression (2) is satisfied, the limiting current is given approxinately by the Bogdankevich and Rukkadze interpolation result

$$
\Delta_{L}^{B R}=\left(r_{0}^{2 / 3}-1.0\right)^{3 / 2 / G\left(R / a_{0}\right)},
$$

where the geometry factor is

$$
G\left(R / a_{0}\right) \equiv 1+2 \ln \left(R / a_{0}\right)
$$

The well-known result (3) is essentially a first iteration result since it does not take into account the radial vartation of the potential. Recent1y, Thompson and Sloan, ${ }^{21}$ and Cenoni and Proctor ${ }^{22}$ have obtained a more exact solution for the limiting current. For a solid beam, the mixed boundary condition

$$
\left[\gamma+\ln \left(R / a_{0}\right) \times \frac{\partial Y}{\partial n}\right]_{r=a_{0}}=\gamma_{0}
$$

is obtained by integrating through the vacuum region to eliminate equation (1b). Combining (la) and (4) yields a single integral equation which can be solved iteratively

$$
\begin{gathered}
\gamma_{K}\left(r / a_{0}\right)=\gamma_{0}-4 \Delta \ln \left(R / a_{0}\right) \int_{0}^{1} \frac{\gamma_{K-1}(y) y d y}{\left(\gamma_{K-1}^{2}-1\right)^{1 / 2}} \\
-4 \Delta \int_{r / a}^{1} \frac{d u}{u} \int_{0}^{u} \frac{\gamma_{K-1}(y) y d y}{\left(\gamma_{K-1}^{2}-1\right)^{1 / 2}},
\end{gathered}
$$


where the subscript denotes the order of the iteration. If the initial guess for $\gamma_{K}=\left(1-\beta_{K}^{2}\right)^{-1 / 2}$ is a constant, then (5) yields the first order results

$$
\begin{aligned}
& \gamma_{1}\left(r / a_{0}\right)=\gamma_{c}+\left(\gamma_{e}-\gamma_{c}\right)\left(r / a_{0}\right)^{2}, \\
& \gamma_{e}=\frac{\gamma_{0}+2 \gamma_{c} \ln \left(R / a_{0}\right)}{G\left(R / a_{0}\right)},
\end{aligned}
$$

and

$$
\Delta=\beta_{K}\left(\gamma_{e}-\gamma_{c}\right)
$$

Here $\gamma_{c}$ and $\gamma_{e}$ are the first order relativistic factors associated with electrons at the center and edge of the beam. From (6) and (7) we note that the concept of a rigid rotor ${ }^{18}$ is only valid for

$$
\gamma_{0}-\gamma_{c} \ll \gamma_{0} G\left(R / a_{0}\right),
$$

which requires $\Delta_{0} \ll \Delta_{L}$.

Finally, Genoni and Proctor have also obtained approximate iterative results for annular beams, as well as a general upper bound for the limiting current. 22 


\section{NUMERICAL METHODS}

Computational results in the following sections were obtained principally from two computer programs, CCUBE (version one) and CRADR. The first of these is a two-dimensional, relativistic, electromagnetic, particle-in-cell plasma simulation code. It treats self-consistantly the time-dependent trajectorius of tens of thousands of individual beam electrons over thousands of plasma periods. At each time step, the charge and current associated with the electrons are interpolated bilinearly onto a spatial mesh. These quantities in turn serve as source terms in Maxwell's equations for determining the electromagnetic putentials on the mesh. Finally, the potentials are interpolated back to the particle positions, and the particle momenta and positions advanced. This leapfrog procedure is repeated many thousands of times and forms the heart of the program.

CCUBE differs from most other plasma simulation codes in two important respects. First, it enploys a partially Galerkin canonical momentum algorithm for advancing particle quantities: Electromagnetic scaler and vector potentials rather than electromagnetic fields are used in the particle equations of motion. ${ }^{37}$ This has the effect of creating a Lagrangian interface between particles and fields, and thereby eliminates the virulent numerical Cherenkov instability ${ }^{38}$ and ameliorates other bothersome numerical problems. It appears also to improve computational accuracy, especially in the ignorable coordinate direction. Secondly, the program-is written for arbitrary separable, orthogonal coordinates. Thus, one can convert from, say, cylindrical to parabolic coordinates 
merely by redefining an array of metric elements. (Parabolic coordinates may, incidentally, be useful in some collective ion acceleration problems. ${ }^{39}$ ) To date only rectangular, cylindrical, and spherical coordinates have actually been employed in simulations. Nonuniform zoning of the spatia! mesh is accomplished with equal ease.

A number of other features deserve mention. For compatibility with nonuniform zoning, variable particle weighting within particle type, with several distinct particle types permitted, is implemented. The program supports both periodic and inhomogeneous Dirichlet-Neumann boundary conditions,

$$
\alpha_{1} \psi+\alpha_{2} \frac{\partial \psi}{\partial n}=\alpha_{3}
$$

on the electromagnetic potentials, and wave transmitting boundaries are not difficult to add. Particles can be absorbed, reflected, or injected from surfaces not necessarily coinciding with edges of the mesh. Diagnostics include microfiche plots of various slices through particle phase space; particle distribution functions; contour plots and one-dimension cuts of the fields, potentials, and currents; and histories of particle and field energies and other selected quantities of j.nterest. Color movies of particle and field data can be generated concurrently. CCUBE is tightly optimized for efficient operation on the CDC-7600.

The spatial configuration for all particle code simulations in this article is shown in Fig. 1. The basic coordinate system is cylindrical, with azimuthal symmetry assumed. A uniform axially directed magnetic is imposed. Radial transformations are utilized to concentrate cells within the beam. Early simulations were carried out with 


$$
\mathrm{x} 1=\mathrm{z} \omega_{\mathrm{b}}^{0} / \mathrm{c}
$$

and

$$
\mathrm{X} 2=0.5\left(\mathrm{a}_{0} \omega_{\mathrm{b}}^{0} / \mathrm{c}\right)^{2} \ln \left[1.0+\left(\mathrm{x} / \mathrm{a}_{0}\right)^{2}\right]
$$

Although this radial transformation proved adequate, later simulations were carried out with

$$
X 1=z \omega_{b}^{0} / c
$$

and

$$
\mathrm{x} 2=\left(\mathrm{a}_{0} \mathrm{w}_{\mathrm{b}}^{0} / \mathrm{c}\right) \ln \left[1.0+\mathrm{r} / \mathrm{a}_{0}\right] .
$$

This latter radial transformation was found to provide a better cell distribution within the beam. In the transformed coordinate system all cells are the same size. The numbers of cells were NX1 $=98$ and $\mathrm{NX2}=34$

Relativistic electrons are injected through a thin foil with a radial distribution of the form

$$
g\left(r, \vec{p}, \theta_{s}\right)=\left[U(r)-U\left(r-a_{0}\right)\right] f_{b}\left(\vec{p}, \theta_{s}\right)
$$

where the local function $f_{b}$ is a scattered beam distribution function discussed in Sec. V. In the limit that the scattering angle $\theta_{\mathbf{s}}$ goes to zero, $f_{b}$ reduces to a Dirac delta function. The injected axial current density is independent of both the particle momentum $\vec{p}$ and scattering angle, but has a finite rise time $\tau_{\mathbf{r}}$

$$
J_{z}(r, t)=J_{z}^{0}\left[U(r)-U\left(r-a_{0}\right)\right]\left[1.0-n\left(t / \tau_{r}\right)\right]
$$


In early simulations $\eta=\exp \left(-t / \tau_{r}\right)$, whereas $\eta=\exp \left(-t^{2} / 2 \tau^{2}\right)$ in the later simulations.

Boundary conditions for the electrons are reflection at the axis and absorption on both the radial and axial surfaces. Potentials are set to zero at the upstream axial and outer rddial boundaries in order to represent metal surfaces. In contrast, normal derivatives of the potentials are set to zero at the downstream end of the simulation grid to mock up an infinitely long cyìinder.

In addition to the traditional simulation diagnostics mentioned previously, CCUBE contains numerical equivalents of such experimental diagnostics as Faraday cups, calorimeters, compensated diamagnetic loops, local probes for field and current measurements, and Rogowski loops. Each diagnestic is time integrated and can be fourier analyzed in time. The code also calculates beam inductance.

GRADR is a much smaller computer program, written to determine the equilibrium profiles and linear eigenmodes of a cylindrically symmetric but radially inhomogeneous, unneutralized, relativistic electron beam in the cold fluid approximation. 40 In cylindrical equilibrium, only $n_{b}^{0}, v_{z}^{0}, v_{\theta}^{0}, B_{z}^{0}, B_{\theta}^{0}$, and $E_{r}^{0}$ are non-zero. Maxwell's equations plus the fluid equations (written in dimensionless form) yield only four relations

$$
\begin{aligned}
& \frac{1}{r} \frac{d}{d r} r B_{\theta}^{0}-n_{b z}^{0} v_{z}^{0}=0 \\
& \frac{d}{d r} B_{z}^{0}-n_{b}^{0} v_{\theta}^{0}=0 \\
& E_{r}^{0}+v_{\theta}^{0} B_{z}^{0}-v_{z}^{0} B_{\theta}^{0}+\frac{\gamma^{0}}{r} v_{\theta}^{0}=0 \\
& \frac{1}{r} \frac{d}{d r} r E_{r}^{0}-n_{b}^{0}=0
\end{aligned}
$$


where $\gamma^{0}$ is the local particle energy. Two of the six quartities can be specified arbitrarily. 18

One must, therefore, choose two physically realizable additional constraints. The first is obvious: Total particle energy, kinetic plus potential, must be constant across the beam and equal to the injection energy.

$$
\gamma^{0}+\int_{r}^{R} E_{r}^{0} d r^{\prime}=\gamma_{0}^{i}
$$

As a second constraint, let the current density be constant at injection. Conservation of canonical angular momentum then determines the current profile within the waveguide,

$$
\mathrm{n}_{b}^{0}(r) r d r=n_{b}^{i}\left(r^{i}\right) r^{i} d r^{i}
$$

and

$$
\frac{1}{2} B_{z}^{i} r^{i^{2}}=\gamma^{0} r v_{\theta}^{0}+\int_{0}^{r} B_{z}^{0} r^{\prime} d r^{\prime}
$$

The system of Eqs. (13)-(16) is solved iteratively, starting from a rigid rotor equilibrium. ${ }^{18}$ convergence is rapid except for currents very near the spacecharge limit. In that case a few thousand iteraticns and a small relaxation parameter are needed to achieve relative accuracy of $10^{-5}$. Of course, for currents above the spacecharge limit no equilibrium exists, and the iterarive process diverges. 
Given an equilibrium determined in this, or any other, way, GRADR then solves the corresponding linearized equations to obtain eigenmodes and eigenvalues, if desired. The linear equations form a fourth order differential system in radius, and are integrated by a standard RungeKutta routine. 41 Muller's method is employed in finding wave frequencies and growth rates. 42 Details are provided in Ref. 40 . 
For an inmersed source, the iterative solution (5) does yield a more accurate estimate for the space charge limiting current for a solid beam. In fact, subject to the assumptions that i) the equilibriur: has no axial dependence, $i i)$ the beam rotation and expansion are negligible, iii) the beam momentum distribution is a Dirac delta function, and iv) the axial current density and beam voltage are constant at the source, we find the iterative solution for $\Delta_{L}$ and $\gamma_{L}$ to be within a few percent of the exact numerical solution calculated by GRADR, where $\gamma_{L}=\gamma_{c}$ at the space charge 1imit. For example, with $\gamma_{0}=2$ and $\mathrm{R} / \mathrm{a}_{0}=2, \Delta_{\mathrm{L}}=0.255$ and $\gamma_{\mathrm{L}}=1.14$ from the iterative solution compared with $\Delta_{L}=0.254$ and $\gamma_{L}=1.16$ from the numerical GRADR solution.

Although the iterative calculation does yield an accurate expression for $\Delta_{L}$ and $\gamma_{L}$, it is sufficiently complex as to require some numerical evaluation. Thus we present in graphical form the quantities $\Delta_{L} / \Delta_{L}^{B R}$ and $\gamma_{L} / \gamma_{L}^{B R}$ as a function of $R / a_{0}$ for various values of $\gamma_{0}$ in Figs. 2 and 3. From this information, a reasonably accurate estimate for $\Delta_{L}$ and $\gamma_{L}$ cani be obtained over a rather wide range of practical parameters. Relative to the essentially first order interpolation result ${ }^{20}$

$$
\Delta_{L}^{\mathrm{BR}}=\left(\gamma_{0}^{2 / 3}-1.0\right)^{3 / 2} / \mathrm{G}\left(\mathrm{R} / \mathrm{a}_{0}\right)
$$

and

$$
\gamma_{L}^{\mathrm{BR}}=\gamma_{0}^{1 / 3},
$$


the maximum deviation occurs for small $\mathrm{k} / \mathrm{a}_{0}$ and $\gamma_{0^{\circ}}$. As discussed in Sec. V, the assumption that the momentun distribution is a Dirac delta function would be difficult to justify experimentally due to anode foil scattering for $350 \mathrm{keV}$ to $1 \mathrm{MeV}$ beams.

Assuming the equilibrium remains axially independent for $\mathrm{B}_{0}^{2} / 8 \pi<\mathrm{n}_{\mathrm{b}}^{0} \mathrm{mc}^{2}\left(\gamma_{0}-1\right)$, the most ill defined assumption in obtaining both the iterative and numerical GRADR solutions shown in Figs. 2 and 3 is the neglect of the beam rotation and associated expansion. Specificaliy, for what level of external magnetic field strength is this assumption valid? To answer this question, we first considered the space charge limiting equilibrium associated with $50 \mathrm{MeV}$ beam as a function of the external magnetic field strength. At the source the radial voltage profiles are $\gamma_{0}(r)=\left[U(r)-U\left(r-a_{0}\right)\right] \gamma_{0}$ and $J_{z}(r)=\left[U(r)-U\left(r-a_{0}\right)\right] J^{0}$ with $R / a_{0}=2.0$. The numerical solution obtained from GRADR is shown in Fig. 4. For $\omega_{c} / \omega_{b}^{0}=2.5,3.0,4.0$, and 14.4 only the space charge 1 imit $\Delta_{L}\left(\omega_{c} / \omega_{b}^{0}\right)$ is calculated, but for $\omega_{c} / \omega_{b}^{0}=2.0,5.0$, and $1000.0 \Delta\left(\omega_{c} / \omega_{b}^{0}\right)$ is shown as a function of $-\mathrm{e} \phi / \mathrm{mc}^{2}$. For comparison the dashed line in Fig. 4 corresponds to the function

$$
\Delta^{B R}=\left(-\mathrm{e} \phi / \mathrm{mc}^{2}\right)\left[1-\left(\gamma_{0}+\mathrm{e} \phi / \mathrm{mc}^{2}\right)^{-2}\right]^{1 / 2} / \mathrm{G}\left(\mathrm{R} / \mathrm{a}_{0}\right)
$$

which maximizes at $-\mathrm{e} \phi / \mathrm{mc}^{2}=\gamma_{0}-\gamma_{0}^{1 / 3}$. Note that the Bogdankevich and Rukhadze ${ }^{20}$ result (17) and the Voronin et a1. ${ }^{27}$ analysis insicate the existence of two equilibria for $\Delta<\Delta_{L}$, the second lying in the narrow band $-\mathrm{e} \phi / \mathrm{mc}^{2}>\gamma_{0}-\gamma_{I}$. No attempt was made with GRADR to find this second branch, because it does not appear to be physically accessible. 
At $\omega_{c} / \omega_{b}^{0}=B_{0}^{2}\left[2\left(\gamma_{0}-1\right)\right]^{1 / 2}=14.4$ the energy density of the external magretic fleld is equal to the beam kinetic energy density, and $\Delta_{L}\left(\omega_{c} / \omega_{b}^{0}\right)$ has increased only slightly. However, as $\omega_{c} / \omega_{b}^{0}$ is reduced below this level, the effects of beam expansion and radial density profile modification are no longer negligible. The radial density profiles are shown as a function of $\omega_{c} / \omega_{b}^{0}$ in Fig. 5 . In addition, the relativistic factor $\gamma_{L}\left(\omega_{c} / \omega_{b}^{0}\right)$ at the beam center, the space charge limiting current $\Delta_{L}\left(\omega_{c} / \omega_{b}^{0}\right) / \Delta_{L}$, the position of the beam edge $a / R$, the maximum rotation velocity $\beta_{\theta}^{m}=v_{\theta}^{m} / c$, and its corresponding position $\mathrm{r}^{\mathrm{m}} / \mathrm{R}$ are summarized in Table $\mathrm{I}$.

Comparing columns 3 and 4 of Table $I$, it is evident that the $1 n-$ crease in $\Delta_{L}\left(\omega_{c} / \omega_{b}^{0}\right)$ is predominantly due to beam expansion rather than the modification in the radial density profile. However, the reduction in $\beta_{\theta}^{m}$ as the magnetic field strength is reduced from $\omega_{c} / \omega_{b}^{0}=2.5$ to $\omega_{c} / \omega_{b}^{0}=2.0$ is the result of the profile modification and increasing value of $\gamma_{L}\left(\omega_{c} / \omega_{b}^{0}\right)$. Finally, we note that for $\omega_{c} / \omega_{b}^{0} \geqslant 5$ the change in $\Delta_{L}\left(\omega_{c} / \omega_{b}^{0}\right)$ from $\Delta_{L}$ is less than $3 \%$. Although this level of magentic field strength remains large $B_{z} \geqslant 109 / a(c m) k G$, it is not impractical. In contrast to $\Delta_{L}\left(\omega_{c} / \omega_{b}^{0}\right)$, the relativistic factor $\gamma_{L}\left(\omega_{c} / \omega_{b}^{0}\right)$ changes substantially from the infinite magnetic field result $\gamma_{L}$ at $\omega_{c} / \omega_{b}^{0}=5.0$. In general, it can be shown that $\omega_{c} / \omega_{b}^{0} \approx 5$ to 10 is the level of magnetic field strength required for the applicability of the space charge limit $\Delta_{L}$ shown in Fig. 2. To proceed, it is clear that the beam must be in the slow mode of rotation, and thus the rotation frequency at any point in the beam interior is approximately 


$$
\omega_{\theta}^{-}=-\left(\omega_{b}^{0}\right)^{2} / 2 \gamma^{2}(r) \omega_{c}
$$

and $Y(r)$ is given by (6). It follows that the rotational velocity is approximately

$$
\beta_{\theta}(r) \simeq 0.5\left(a_{0} \omega_{b}^{0} / c\right)\left(\omega_{b}^{0} / \omega_{c}\right)\left(r / a_{0}\right) /\left[\gamma_{L}+\left(\gamma_{e}-\gamma_{L}\right)\left(r / a_{0}\right)^{2}\right]
$$

which maximizes at

$$
r^{m / a_{0}}=\min \left\{\begin{array}{l}
1 \\
{\left[\frac{\gamma_{L} G\left(R / a_{0}\right)}{3\left(\gamma_{0}-\gamma_{L}\right)}\right]^{1 / 2} .}
\end{array}\right.
$$

Thus, for $\Delta \simeq \Delta_{L}$ low voltage beams tend to rotate fastest at $r^{m} \simeq a_{0}$, whereas the maximum rotation occurs at $r^{m} \ll a_{0}$ for high voltage beams. Noting that $a_{0} \omega_{b}^{0} / c \simeq 2 \Delta^{1 / 2}$ for a solid beam, expressions (19) and (20) can be combined to yield

$$
\omega_{c} / \omega_{b}^{0}=(3 \sqrt{3} / 16) H_{L}\left(\gamma_{0}, R / a_{0}\right) / \beta_{\theta}^{m}
$$

where

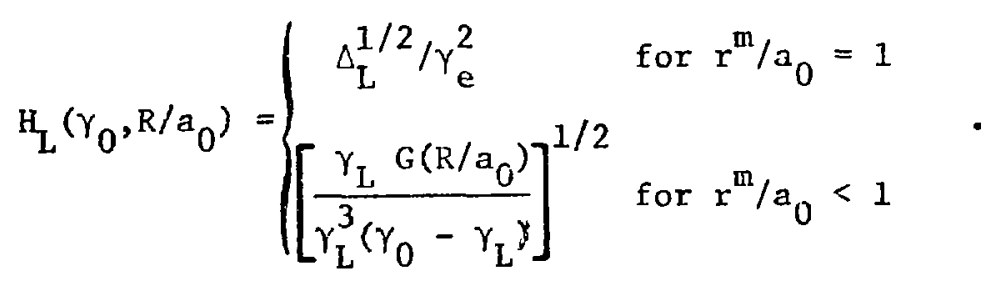

The function $H_{L}\left(\gamma_{0}, R / a_{0}\right)$ is actually a weak function of $\omega_{c} / \omega_{b}^{0}$ through $\gamma_{L}$ 
and $\gamma_{\mathbf{e}^{-}}$However, it has an upper bound of unity. Thus, the magnetic field requirement becomes

$$
\omega_{c} / \omega_{b}^{0} \simeq(3 \sqrt{3} / 16) / \beta_{\theta}^{m} \simeq 5 \text { to } 10
$$

where $\beta_{\theta}^{m}<(3-7) \cdot 10^{-2}$ to limit beam expansion.

Expression (21) is the magnetic field requirement at the space charge 1imit. For a beam source voltage above $5 \mathrm{MeV}$, the relationship between $\Delta$ and $\gamma_{c}=\gamma_{0}+$ e $\phi / \mathrm{mc}^{2}$ below the space charge limit is approximately

$$
\Delta=\Delta_{L} \frac{\left(\gamma_{0}-\gamma_{c}\right)}{\left(\gamma_{0}-\gamma_{L}\right)},
$$

see Fig. 4. Thus, the function

$$
H\left(\gamma_{0}, R / a_{0}\right) \simeq H_{L}\left(\gamma_{0}, R / a_{0}\right)\left(\gamma_{L} / \gamma_{c}\right)^{3 / 2}
$$

and

$$
\omega_{c} / \omega_{b}^{0} \simeq(3 \sqrt{3} / 16)\left(\gamma_{L} / \gamma_{c}\right)^{3 / 2} / \beta_{\theta}^{m}
$$

Consequently, a reduction in the current of only a few percent below the space charge limit $\Delta_{L}$ can result in a significant reduction in either the magnetic field requirement $\omega_{c} / \omega_{b}^{0}$ or the maximum rotational velocity $\beta_{\theta^{*}}^{m}$. For example, consider the $50 \mathrm{MeV}$ case shown in Fig. 4 with $\omega_{c} / \omega_{b}^{0}=14.4$ and $\gamma_{L} \simeq 1.74$. Operating at $\Delta=0.95 \Delta_{L}$ yields $\gamma_{c} \simeq 7.50$, which for fixed $\omega_{c} / \omega_{b}^{0}$ leads to a reduction of approximately 
an order of magnitude in $\beta_{\theta}^{\mathrm{m}}$. This character is shown in Fig. 6. Physically, this is simply the result of the balance between the radial electric field $\mathrm{E}_{\mathbf{r}}$ and the radial force associated with the self magnetic field $-v_{z} B_{\theta}$

The preceeding analysis still assumes that the beam equilibrium has no axial dependence. However, in the study of autoresonant acceleration, two-dimersional axial symmetric CCUBE particle simulations indicate that any radial force discontinuity leads to the development of an equilibrium which is, in fact, axially dependent. 3,39 Specifically, a periodic standing wave pattern with a wavelength of

$$
\lambda \simeq \pi\left(c_{b}^{0} / \omega_{c}\right) \gamma_{e} \beta_{e} e_{0} / \Delta^{1 / 2}
$$

is formed. These zero frequency perturbations correspond to the beam resonance $\omega \simeq k_{z} v_{z}-\omega_{c} / Y_{e}$. The formation of this axially dependent equiljbrium can be caused by i) the initial stace not being sufficiently close to the equilibrium state, ii) a discontinuity in the radial electric field due to the anode foil, iii) a discontinuity in the external magnetic field, or iv) a discontinuity in the drift tube radius.

For wavelengths $\lambda$ short compared with the beam radius $a_{0}$, we expect the axially independent solutions to be approximately correct. However, for $\lambda \sim a_{0}$, a solution for $\Delta_{L}$ based upon the axially independent equilibrium assumption may be in error. To investigate this point we have performed a series of simulations in which $\lambda / a_{0} \sim 0(1)$, the results of which are summarized in Tabje II. The basic parameters 
are $\gamma_{0}=5, \mathrm{k} / \mathrm{a}_{0}=2.0$, and $\mathrm{L} / \mathrm{a}_{0}=12.0$. A fixed mignetic field strength of $\omega_{c} / \omega_{b}^{0}=4.0$ was used for the entire series. The cuordinate transformation (9) was used. At constant voltage, the injected current was given a finite rise time of

$$
\Delta_{I}(t)=\Delta_{I}\left[1-\exp \left(-\omega_{b}^{0} t / 12.0\right)\right],
$$

with a local beam distribution function

$$
f_{b}=\delta\left(p_{\mid}-p_{0}\right) \delta\left(p_{1}^{2}\right) / 2 \pi
$$

In Fig. 7 the numerical solution for $\Delta\left(\omega_{c} / \omega_{b}^{0}\right)$ calculated from GRADR for $\omega_{c} / \omega_{b}^{0}=4.0$ is depicted by the dashed 1ine. For comparison the infinite magnetic field space charge limit $\Delta_{L}$ and the curve $\Delta^{B R}$ are also shown. Note that the ratio of $\Delta_{\mathrm{L}}(4.0) / \Delta_{\mathrm{L}}=1.05$ is essentially the same as found for the $50 \mathrm{MeV}$ beam, Table I, consistent with expression (21). The simulation results for injection currents below the space charge limit are represented by solid dots, with the error bar representing the approximate level of error for the estimate of $-e \phi(r=0) / m c^{2}$. At the time of these runs, late 1975, the diagnostic package on the code was still in development, and $-\mathrm{e} \phi(\mathrm{r}=0) / \mathrm{mc}{ }^{2}$ had to be estimated from contour plots. The simulation results A1 through A6 are in agreesient with the numerical GRADR solution $\Delta(4.0)$, despite the presence of a periodic axially dependent equilibrium. For injection currents exceeding the space charge limit, runs A7 through A11, the average transmitted current exceeds $\Lambda_{L}$. These runs are represented by the open squares in Fig. 7. We defer the discussion of these runs until the end of the section. 
In Fig. 8 an example of the statinary periodic arially dependent equilibrium is shown, run $A 4$ in Table II. The relavent transformations are $X !=2 \omega_{b}^{0} / c$ and $x 2=2.0 \ln \left[1.0+\left(r / a_{0}\right)^{2}\right]$. For $\gamma_{e}$ and $\Delta(4.0)$ determined from CRADR, expression (23) yields $\lambda / a_{0}=2.8$ which is good agreement with the CCUBE simulation result $\lambda / a_{0}=2.71$.

The amplitude of the surface modulation shown in Fig. 8 can be es: imated using conservation of canonical angular momentum

$$
\gamma(r) m v_{\theta}(r)-\frac{e}{2 c} B_{0} r=-\frac{e}{2 c} B_{0} a_{0}
$$

and radial force balance at the mintmum beam radius $r=r_{m}$

$$
B_{\theta}\left(r_{m}\right) B_{0} \simeq\left\{\begin{array}{ll}
\beta_{e} B_{0} / \gamma_{e}^{2} & E_{r} \text { not shorted } \\
\beta_{e} B_{\theta} & E_{r} \text { shorted }
\end{array} .\right.
$$

For a solid density beam produced by a foil diode, as shown in Fig. 8, the change in beam radius $\delta r=a_{0}-r_{m}$ is

$$
\frac{\delta r}{a_{0}}=\beta_{e}^{2} \gamma_{e}\left(\omega_{b}^{0} / \omega_{c}\right)^{2}
$$

In addition, the magnitude of the velocity associated with this modulation is

$$
\beta_{\mathrm{T}}^{\mathrm{m}} \simeq \beta_{\mathrm{e}}^{2}\left(\omega_{\mathrm{b}}^{0} / \omega_{c}\right) \Delta^{1 / 2}
$$

Note that $\beta_{T}^{m}$ is not a precessional velocity, but is the nagnitude of a periodic twisting velocity 


$$
\begin{aligned}
& B_{\theta}(t)=-\beta_{T}^{m} \sin (2 \pi z / \lambda) \\
& B_{r}(t)=-\beta_{T}^{m} \cos (2 \pi z / \lambda),
\end{aligned}
$$

as show in F1g. 9. Roughly speaking, this motion is superimposed upon the precessioned $\bar{E} \times \bar{B}$ motion. Expressions (27) through (29) are valid for sma1.1 amplitude modulations. These expressions are approximate Iy 30\% low when compared with the modulation amplitude in Fig. 8 and the azimuthal particle angular momentum $\gamma(r) r B_{\theta}(r)$ in $F i g .9 b$.

With such a complicated motion, the interior and exterior of the beam cannot remain in phase over a certain length. In fact, phase mixing of the beam can be seen in Fig. 8. The distance $L_{c}$ over which the laminar beam character can be retained is approximately

$$
L_{c} / a_{0} \simeq \pi\left(\omega_{b}^{0} / \omega_{c}\right)\left(\gamma_{e}^{2} / \Delta\right) /\left(\beta_{e}-\beta_{c}\right)
$$

for $\beta_{T}^{m} \gg \beta_{0}^{m}, \beta_{e}=\left(1-\gamma_{e}^{-2}\right)^{1 / 2}$, and $\beta_{c}=\left(1-\gamma_{c}^{-2}\right)^{1 / 2}$. Huwever, as $\delta \mathrm{r} / \mathrm{a}_{0} \rightarrow 0$, the coherence length $\mathrm{L}_{c}$ may be longer than that given by (30).

On the basis of the analytic expression (21), the GRADR solutions for $\Delta_{L}\left(\omega_{c} / \omega_{b}^{0} \geqslant 5\right)$, and the series of simulations summarized in Table II, we conclude that $\Delta_{L}$ shown in Fig. 2 is valid for $\omega_{c} / \omega_{b}^{0} \geqslant 5.0$. However, the initial beam equilibrium has a periodic dependence in the direction of propagation with local angular and radial velocities of up to $0.1 \mathrm{c}$.

Strictly speaking, the preceeding conclusions apply only to solid beams generated by foil dlodes. We do note, however, that the equilibrlum of a high voltage beam generated by a follless diode nas the 
potential of being significantly more laminar than that of a high voltage beam generated by a foil diode, on the basis of expression (26).

Since a significant iraction of the experimental data has not been attained with ${ }_{c} / u_{b}^{0} \geqslant 5$, we have performed a second series of simulations in which the injection current is fixed and the magnetio field strengtin is varied down to $\omega_{c} / \omega_{b}^{0}=0.0$. This second series of simulations is summarized in Table IIT. The injected beam distribution function and current rise time are as described earlier, expressions (24) and (25). However, the basic parameters are $\gamma_{\theta}=3.0, R / a_{0}=7.39$, $\mathrm{L} / \mathrm{a}_{0}=22.2,{ }^{a}{ }_{0} \omega_{b}^{0} / c=1.117$, and $\Delta_{I}=0.292$. Except for runs B1, B2, and $B 5$ in which $x 2=\left(a_{0} \omega_{p}^{0} / c\right) \ln \left[1.0+r / a_{0}\right]$, the radial coordinate transformation is given by (9).

In Fig. 10 the transmitted current $\Delta_{\mathrm{T}}$, column 3 of Table III, is given as a function of the magnetic field strength $\omega_{c} / \omega_{b}^{0}$. The horizontal dashed line corresponds to the Bogdankevich and Rukhadze ${ }^{20}$ result $\Delta_{L}^{B R}$ given by expression (2). We have als s calculated $\Lambda_{L}\left(\omega_{c} / \omega_{b}^{0}\right)$ using GRADR, which is the solid line. As expected, only runs B2 and B3 in which $\omega_{c} / \omega_{b}^{0}=5.0$ have transmitted currents within a few percent of the infinite magnetic field result $\Delta_{\mathrm{L}}$. We also note that the GRADR solution for $\Delta_{L}\left(\omega_{c} / \omega_{b}^{0}\right)$ does not agree with the simulation results below $\omega_{c} / \omega_{b}^{0}=4 \cdot 0-5 \cdot 0$.

In Figs. $11(a)$ and $11(b)$ beam configuration space is shown for $\omega_{c} / \omega_{b}^{0}=5.0$ and $\omega_{c} / \omega_{b}^{0}=2.00$. At a magnetic field strength of $\omega_{c} / \omega_{b}^{0}=5.0$ little expansion of the bean is evident and only a slight short wavelength ripple appears on the beam surface. Since the electrons are 
essentially tied to the field lines, the center of the beam is totally reflected where the radial potential is maximum, and the transmitted current is annular. In this context, the transmitted current was also observed to be annular for runs $A 7$ through $A 11$ where $\omega_{c} / \omega_{b}^{0}=4.0$. When the magnetic field is reduced to $\omega_{c} / \omega_{b}^{0}=2.00$, the beam expands rapidly since the injection conditions become farther from equilibrium. At injection the electrons have no $v_{\theta}$ component and thus no $v_{\theta} B_{z}$ restoring force exists. As electrons move away from the anode foil, the net outward force $E_{r}-v_{2}^{B} \theta$ causes the beam to expand and overshoot its equilibrium radius. At that point, the $v_{\theta} B_{z}$ restoring force exceeds $E_{r}-v_{z} B_{\theta}$ and electrons move inward. This oscillatory behavior continues until the electrons have moved a distance $\mathrm{L}_{\mathrm{c}}$ from the anode at which point the oscillations have phase mixed away, as seen in Fig. 11(b). Under such conditions, the axially independent equilibrium assumption implicit in GRADR cannot be expected to be correct.

As the magnetic field strength is decreased below $\omega_{c} / \omega_{b}^{0}=2.00$, the radial expansion becomes increasingly violent and ultimately the beam hits the drift tube wall. In the present set of simulations in which $R / a_{0}=7.39$ was large, this occurred at $\omega_{c} / \omega_{b}^{0} \sim 1.0$. Until the beam hits the wall, the transmitted current will increase as the magnetic field strength is reduced.

The observation of such a dramatic change in beam equilibrium as the magnetic field strength is reduced leads to an interesting question of what transpires when the so-called stability condition 19

$$
\left(\omega_{b}\right)^{2}<2 \gamma \omega_{c} c / R
$$


is violated. We make three relevant observations concerning this condition. Firstly, it is based upon a rigid rotor equilibrium which we have found to have very linited validity. Secondly, it is a sufficient, rather than necessary, condition for stability. An examination of the original variational argument which led to the condition demonstrates that it may be violated quite strongly without necessarily leading to instability. In fact, taking into account radial beam expansion, situations in which violation of (31) is sufficiently strong to give rise to instability may not be of direct experimental interest. Thirdly, the condition obviously arises only when electromagnetic effects are included. Electrostatic stability is assured by the simple requirement: that the rigid-rotor distribution function be a monotonically decreasing function of its argument. ${ }^{43}$ Therefore, it seems reasonable to assume that instability resulting from the violation of the condition is clue to the intersection of a light wave dispersion curve with that of a beam mode.

The combination of radial inhomogeneity and cther seif-field effects seem to render the linear theory problem intractable from a purely analytic point of view. However, the numer scal code GRADR is a.1so capable of performing stability analyses about specified or self-consistent equilibria. In an attempt to arrive at a preliminary answer to the question raised above, we have employed this code to examine several equilibria in selected regions of mode space. The firşt equilibrlum, as described previously, was one constructed self-consistently 
as described to satisfy the space charge flow crfteria, and only weakly violated the stability condition. Regions of mode space chosen for study were those in which the simple form of the dispersion relation Indicated that one would find a beam mode. Azimuthal mode numbers of $\ell=0,1,2$ were examined for this case. No instability was observed. Consequently, we then turned to a rigid rotor equilibrium which strongly vlolated the stability condition, but which did no: provide a selfconsistent solution to the equilibrium equations. Again the $\ell=0$ and 1 modes appeared to be stable. For $\ell=2$, however, a very weak Instability $\delta \approx\left(10^{-4}-10^{-5}\right) \omega{ }_{b}^{0}$ was observed in a reginn of $w-k$ space that was thought to contain a slow cyclotron wave. We have as yet not been able to solate the light wave with which this mode is intersect. ing. Clearly, further study is required. However, we believe that this brief analysis indicates that, for regions of parameter space of practical interest, no virulent instabilities are expected from a factor of ten violation of the stability condition, if at all.

In the first set of simulations, which are summarized in Table II, we observed that when $t^{2}$.e injected current exceeded the theoretical space charge current, the average transmitted current actually exceeded the theoretical limiting current. Shortly after the formation of the virtual cathode; both its position and magnitude evolved into an oscillating steady state. The frequency of oscillation was $\omega_{v} \approx(1.09-1.12) \omega_{b}^{0}$. In the second set of simulations the virtual cathode remained relatively stationayy in time; with no discernible increase of the transmitted current above the theoretical value. of course, the injected current was only slightly above the theoretical limit for this series, 
and there was some indication from the first series of simulations that the time required for the virtual cathode to develop its oscillatory state depended upon how far the injected current was above the theoretical limit. Thus, it is possible that the time scale for these simulations was too short relative to the time needed for the development of the oscillatory state.

Rather than cxtend the time scale of the preceeding simulations, we performed a single additional simulation in which the injected current greatly exceeded the theoretical limiting current. A summary of this simulation is given in Table IV. The parameters chosen are similar to the Prono et al. experiment. 44 The dimensionless injected current for this simulation was $\Delta_{I}=3.12$. From the net current loop located at $\mathrm{X} 1=23.20 \mathrm{c} / \mathrm{u}_{\mathrm{b}}^{0}$ shown in Fig. 12 , it is clear that tile maximum instantaneous transmitted current only reached about $20 \%$ of the injected current. However, the average transmitted current $\left(t=70 \omega_{b}^{-1}\right.$ to $\left.t=122 \omega_{b}^{-1}\right)$ was $12 \%$ above the theoretical limit, compare columns 6 and 7 of Table IV. The transmitted current profile was annular with $\delta a / a_{0} \simeq 0.25$.

The osciliatory stcady state of the virtual cathode developed rapidly, as shown by the net current loop located at $X 1=1.35 \mathrm{c} / \omega_{\mathrm{b}}^{0}$ in Fig. 13. The oscillatory frequency was found to be $\omega_{v} \approx(1.59-1.65) \omega_{b}^{0}$, with the position of the maximum potential varying between $\mathrm{Xl} \simeq c / \omega_{b}^{0}$ and $x 1=(1.5-2.0) c / \omega_{b}^{0}$.

Based upon this simulation, it would appear that $\omega_{v}=\left(\Delta_{I} / \Delta_{L}\right)^{1 / 4} \omega_{b}^{0}$. We also conclude that the time for the virtual cathode to develop its oscillatory state and its average position from 
the anode foll both decrease as $\Delta_{I} / \Lambda_{L}$ increases. Finally, the average transmitted current can be enhanced slightly above the theoretical time Independent limiting current as ? result of the oscillation of the virtual cathode. 


\section{SCATTERED BFAY THEORY}

In most experinents investigating vactium propagation of a relativistic beam the electrons pass through a thin ande foil before entering the drift tube. In plasma heating, for example, it is well known that the scattering incurred by the beam electrons in passing through such a foil can significantly effect the interaction. ${ }^{45}$ Consequently, in the present section, we develop a model to investigate the possible modification of the space charge limiting current due to anode foil scattering.

For scattering angles up to $\theta_{s}=\pi / 5$, the relativistic electrons lose a negligible fraction of their energy in passing through the anode foil. Thus, the local beam momentum distribution function is approximately

$$
f_{b}(p, \theta)=\frac{\delta\left(p-p_{0}\right) \exp \left(-\sin ^{2} \theta / \sin ^{2} \theta_{s}\right)}{2 \pi p_{0}^{2} \sin ^{2} \theta_{s} D\left(1 / \sin \theta_{s}\right)} .
$$

The distribution is normalized to unity

$$
2 \pi \int_{-\infty}^{m} d p|| \int_{0}^{\infty} f_{b}(\vec{p}) p_{1} d p_{1}=1, p_{1}=p_{\theta} \sin \theta,
$$

and $D(y)=\exp \left(-y^{2}\right) \int_{0}^{y} \exp \left(u^{2}\right) d u$ is Dawson's integral. ${ }^{41}$ Ás discussed by Bethe, ${ }^{46}$ the Molière theory of multiple scattering yields an equivalent $1 /$ e scattering angle of

$$
\theta_{s}=\chi_{c}(B-1.0)^{1 / 2}
$$

The quantity $B$, which is typically 5 to 10 , is related to the number of scattering events 


$$
N_{s}=\ln \left\{\frac{6680 \rho x}{\beta_{0}^{2}}\left[\frac{(2+1) 2^{1 / 3}}{\Lambda\left(1.0+3.34 z^{2} x^{2} / \beta_{0}^{2}\right)}\right] \mid\right.
$$

through the transiormation $b-2 n(B)=N_{S}$. De have defined 0 as the foll density in $\mathrm{gm}^{\mathrm{cm}} \mathrm{cm}^{3}, \mathrm{x}$ as the foil thickness in $\mathrm{cm}, \mathrm{A}$ as the atomic number, $Z$ as the number of electrons per atom, and $\alpha=\hbar c / e^{2}$ as the fine structure constant. The angle

$$
X_{c}=\left[4 \pi N Z(Z+1) r_{0}^{2} \times / \beta_{0}^{2} \gamma_{0}^{2}\right]^{1 / 2}
$$

f.s due to Rutherford scattering, where $\mathrm{N}$ is the number of foil atoms per $\mathrm{cm}^{3}$ and, $\mathrm{r}_{0}=\mathrm{e}^{2} / \mathrm{mc}^{2}$ is the classical electron radius.

To calculate the effect of the scattering on the space charge limit, we split the beam into $M$ separate beams each characterized by an angle $\theta_{i}$, axial velocity $v_{i}$, and density $n_{i}$. The potential at the center of a constant density beam is then

$$
\phi=-I \frac{\sum_{i=1}^{M} n_{i}}{\sum_{i=1}^{M}\left(v_{i} n_{i}\right)} G(R / a),
$$
where $I=e \sum_{i=1}^{M}\left(v_{i} n_{i}\right)$ is the total beam current. From conservation of

$$
v_{i}=c\left\{1-\beta_{0}^{2} \sin ^{2} \theta_{1}-\left(\gamma_{0}+e \phi / m c^{2}\right)^{-2}\right\}^{1 / 2}
$$

Combining expressions (34) and (35), it follows that 


$$
P_{M}\left(\phi, \theta_{s}\right) \equiv-\left(c / m c^{2}\right) \sum_{i=1}^{M} w_{i}\left[a_{i}-\left(\gamma_{0}+e \cdot \phi / m c^{2}\right)^{-2}\right]^{1 / 2}=\frac{e I}{m c^{3}} G\left(R / a_{0}\right),
$$

The fraction of the beam with angles between $\partial_{i}$ and $\partial_{i}+\pi / 2 M$ is $w_{i}=n_{i} / n_{b}^{0}=(\eta / 2 M) \exp \left(-\sin ^{2} \theta_{i} / \sin ^{2} \theta_{s}\right)\left(\sin \theta_{i} / \sin \theta_{s}\right) D\left(1 / \sin \theta_{s}\right)$ and $a_{i}=1.0-\beta_{0}^{2} \sin ^{2} \theta_{i}$. Setting $\theta_{i}=0, P_{M}\left(\phi, \theta_{s}\right)$ reduces to expression (2) sinre $\sum_{i=1}^{M} w_{i}=1$. Alternatively, letting $\theta_{i}=\theta_{s}$ yields

$$
\Delta_{L}^{l}\left(\infty, \theta_{s}\right)=\left[\left(\sqrt{a_{s}} \gamma_{0}\right)^{2 / 3}-1.0\right]^{3 / 2} / G\left(R / a_{0}\right)
$$

with

$$
-e \phi / m c^{2}=\gamma_{0}-\left(\gamma_{0} / a_{s}\right)^{1 / 3} \quad .
$$

Finally, ${ }_{M}\left(\phi, g_{S}\right)$ can be computed numerically to determine the space charge limiting current as a function of the foil scattering angle

$$
\Delta_{L}^{M}\left(\infty, \theta_{s}\right)=P_{M}^{\max }\left(\theta_{s}\right) / G\left(R / a_{0}\right)
$$

In Fig. 14 both $\Delta_{L}^{1}$ (solid 1ine) and $\Delta_{L}^{M}$ (dashed line) are shown as a function of $\theta_{s}$ for $\gamma_{0}=3.0$ and $R / a_{0}=7.39$. We also show the results of three simulations, runs D1 through D3, which are summarized in Table V. The basic parameters for this series of simulations are the same as for series C, Table IV. However, the injection distribution function is

$$
g(p, \theta, r)=\left[U(r)-U\left(r-a_{0}\right)\right] f_{b}\left(\vec{p}, \theta_{s}\right),
$$


where $f_{b} 15$ given by (32). The injected current remains independent of the scattering angle; see expression (12).

Overall the simulation results are in good agreement with both $A_{L}^{I}\left(\infty, \theta_{s}\right)$ and $\Delta_{L}^{M}\left(\infty, \theta_{s}\right)$. Note that the transmitted current always exceeds the more exact expression $L_{L}^{M}\left(\infty, \theta_{s}\right)$ with the largest error at $\theta_{s}=0$. This discrepancy is assoclated with the neglect of the radial dependence of the potential. As the potential decreases for increasing $\theta_{s}$, given by column 4 in Table $V$, an improved agreement between the simulation results and expression (38) at large angle scattering would be expected. Since these simulations were performed with a finite magnetic field of $\omega_{c} / \omega_{b}^{0}=5.0$, the slight increase in the transmitted current relative to $\Delta_{L}^{M}\left(\infty, \theta_{s}\right)$ at $\theta_{s}=30^{\circ}$ is consistent with bean expansion.

Using the approximate expression (37), a criterion for the neglect of foll scattering on the space charge limit can be obtained. Assuming $\sin \theta_{s}=\theta_{s}=x_{c}(B-1.0)^{1 / 2}$, we obtain

$$
\begin{aligned}
\Delta_{L}^{1}\left(\infty, \theta_{s}\right) & =\left\{\left[\gamma_{0}^{2}-\gamma_{0}^{2} \beta_{0}^{2} \chi_{c}^{2}(B-1.0)\right]^{2 / 3}-1.0\right\}^{3 / 2} / G\left(R / a_{0}\right) \\
& =\left\{\left[\gamma_{0}^{2}-F\right]^{2 / 3}-1.0\right\}^{3 / 2} / G\left(R / a_{0}\right)
\end{aligned}
$$

For $B_{0} \simeq 1$, the foil scattering function ${ }^{10}$

$$
F \equiv 4 \pi \mathrm{NZ}(Z+1)={ }_{0}^{2} \mathrm{x}\left[\mathrm{B}\left(\mathrm{B}_{0}=1\right)-1.0\right]
$$

depends only on the foil parameters. In Table VI, some typical values for F are glven. It follows from expressions (38) and (39) that foil scattering has a negligible ( 1\%) effect on the space charge limit if

$$
\gamma_{0}^{4 / 3}\left(\gamma_{0}^{2 / 3}-1.0\right)>100 \mathrm{~F}
$$


If criterion (40) is not satisfied, expression (38) must be used to estimate the reduction in $\Delta_{L}$ due to foil scattering. For beam voltages below $i \mathrm{MeV}$, it will be difficult to obtain experimental results which are independent of foil scattering. 
VI. CORRELATIONS BETWEEN FXPERIMENT AND THEORY

A. Abrashitov et a1. ${ }^{34}$

In this experiment a beam with a peak electron voltage of approximately $1 \mathrm{MeV}$ passed through a $50 \mu \mathrm{m}$ titanium anode foil before entering a vacuum chamber. From Table VI, $F \simeq 0.913$ and criterion (40) is not satisfied. Thus, the effect of the foll scattering on the space charge limiting was not negligible in this experiment. Using expression (33) we estimate the scattering angle to be $\theta_{S} \simeq 20^{\circ}$.

The peak beam current was measured to be $I=5$ to $6 \mathrm{kA}$, with a cathode radius of $a_{0}=1 \mathrm{~cm}$. For comparison, we have assumed a constant density beam of total current $I=5 \mathrm{kA}$. It follows that $a_{0} \omega_{b} / c=1.117$ at the source, which is the dimensionless beam radius used for both simulation series $C$ and $D$. In fact, series $D$ is an attempt to reproduce the major results of the Abrashitov et al. ${ }^{34}$ experiment. Specifically: we investigate $i$ ) the transmitted current for a strong magnetic Field, ii) the transmitted energy measured by a calorimeter as a function of the external magnetic field strength, and iii) the diamagnetic loop measurements.

In attempting to simulate this experiment, we found it difficult to determine the radius of the zeturn conductor in the vacuum region from the anode foil to the beginning of the glass tube of internal diameter of $11 \mathrm{~cm}$ and length $220 \mathrm{~cm}$; see Fig. I of Ref. 34. In addition, the magnitude of the external axial magnetic field dropped by a factor of 1.7 In this region. The fact that the experiment was conducted in this fashion is unfortunate, as it is not clear where the virtual cathode actually formed. On the basis of previous simulations, however, we 
expected the virtual cathode to form at an axial distance between $z \approx R / 2$ and $z \approx R$. We estimated the radius of the four return conductors, which were outside the diamagnetic loops of radius $6.5 \mathrm{~cm}$, to be 7 to 9 cri. For an axial magnetic field dependence of $B_{z}(z) \simeq\left\{1.0+0.7\left[1.0+(z / d)^{2}\right]^{-3 / 2}\right\}$, the local magnetic field strength is then $B_{z}(R) \simeq(1.29-1.39) B_{0}$ and $B_{z}(R / 2) \simeq(1.53-1.60) B_{0}$, where $B_{0}$ is the strength of the magnetic field at the center "fi the solenoid section and the radius of the mirror coil is $\mathrm{d}=10 \mathrm{~cm}$. Assuming the beam expands adiabatically, it follows that $\mathrm{R} / \mathrm{a}=7.43 \pm 1.11$. The error in the geometry factor is then about 6 percent $G=5.01 \pm 0.30$. Based upon the above estimate, we actually choose to simulate a system with $\mathrm{R} / \mathrm{a}=7.39$. In order to facilitate comparison with series $\mathrm{C}$ simulations, a uniform magnetic field was imposed, rather than a mirror. The results of the simulations are summarized in Table $V$. A new radial transformation was used $\mathrm{X} 2=1.117 \ln \left(1.0+\mathrm{r} / \mathrm{a}_{0}\right)$ which improved the distribution of cells inside the beam. As with previous runs, no axial transformation was performed $\mathrm{X1}=\mathrm{z} \mathrm{w}_{\mathrm{b}}^{0} / \mathrm{c}$. Runs $\mathrm{D} 1$ through $\mathrm{D} 3$ were discussed previousiy in Sec. V, as was the injection distribution expressions (32) and (12). The total injection current was $\Delta_{I}=0.275$ for this series.

In the strong magnetic field regime, the experimental limiting current was approximately $I_{L} \simeq 3 \mathrm{kA}$. The limiting current obtained from simulation $\mathrm{D} 2$ is

$$
\begin{aligned}
I_{L} & =17.0 \Delta_{L}(5.0,20.0) \mathrm{kA} \\
& =3.38 \pm 0.20 \mathrm{kA}
\end{aligned}
$$


whereas expression (38) predicts

$$
I_{L}=3.35 \pm 0.20 \mathrm{kA}
$$

Considering the unknowns, this is good agreement between experiment, simulation, and theory. For comparison, the liniting current for an unscattered beam is $I_{L}=4.21 \pm 0.25 \mathrm{kA}$.

In Fig. 2 of Ref. 34 the beam energy $Q\left(B_{z}\right)$ measured by a $6 \mathrm{~cm}$ diameter calorineter positioned at the end of the vacuum drift tube is plotted as a function of the external magnetic field strensth. In order to compare these data with our simulation results, we have plotted the guantity $Q\left(B_{z}\right) / Q_{0}$, represented by solid dots, as a function of $B_{z}$ in Fig. 15. Here $Q_{0}$ is the measured beam energy at $B_{z}=12.65 \mathrm{~kg}$. Also in Fig. 15, we have plotted the simulation results $Q\left(B_{z}\right) / Q_{0}$, where $Q_{0}$ is the energy collected by a $6 \mathrm{~cm}$ diameter calorimeter at $\omega_{c} / \omega_{b}^{0}=5.0$. Both the experimental and simulation data show a sharp decrease in apparent transported energy for $B_{0}<3.5 \mathrm{kG}$. This decrease is the result of the beam expanding to an equilibriun radius which is larger than the calorimeter. As opposed to the simulation data, the experimental data siows a slight decrease as the magnetic field strength is reduced from $\mathrm{B}_{0}=7 \mathrm{kG}$ to $\mathrm{B}_{0}=3.5 \mathrm{kG}$. This discrepancy we attribute to an increasing fraction of the beam being reflected by the mirror at the end of the Arift tube. Note that the position of the virtual cathode occurs farther from the entrance foil as the magnetic field is lowered, see columi 5 in Table v. Since the virtual cathode produces a nonadiabatic increase in transverse kinetic energy of the transmitted bean, the fraction of the 
beam reflected increases as the effective strength of the mirror field Increases. Consistent with this interpretation is the rather significant decrease in beam inductance (open circles) shown in Fig. 15. Coupled with conservation of angular momentum, this beam expansion implies an increase in transverse beam energy.

As a final point, we consider the diamagnetic loop signals shown in Figs. 3(c) and 3(d) of Ref. 34. The loops were located at 35 and $90 \mathrm{~cm}$ from the anode foil, and we estimate their magnitude to be $W_{1}=(1.3-1.5) \times 10^{17} \mathrm{eV} / \mathrm{cm}$ and $W_{\perp}=(5.0-7.0) \times 10^{16} \mathrm{eV} / \mathrm{cm}$, respective1y. Assuming adiabatic beam expansion through the mirror, the stated magnetic field of $7 \mathrm{kG}$ corresponds to $\omega_{c} / \omega_{b}=5.0$ in the solenoid region. This value of magnetic field strength is consistent with simulations Di through D3.

To analyze the diamagnetic loop signal, we assume existence of a magnetic flux conserving surface of radius $R$. Furthermore, the beam equilibrium is assumed to be azimuthally symmetric with no axial dependence. Conservation of particle and field momentum then yeilds

$$
\Delta B_{z}^{+}=-\frac{2}{R^{2}-a^{2}} \int_{0}^{a} \Delta B_{z}(r) r d r
$$

We have defined the change in the local magnetic field strength as $\Delta \mathrm{B}_{\mathrm{z}}^{-}=\mathrm{B}_{\mathrm{z}}(\mathrm{r})-\mathrm{B}_{0}$ when $\mathrm{r} \leqslant \mathrm{a}$ and $\Delta \mathrm{B}_{\mathrm{z}}^{+}$for $\mathrm{r}>\mathrm{a}$. The constancy of $\Delta \mathrm{B}_{\mathrm{z}}^{+}$ in the vacuum region $r>$ a follows from conservation of field momentum. For a diamagnetic loop located at a radius $a<b<R$, the change in flux is 


$$
\begin{aligned}
\Delta \Phi^{b} & =2 \pi \int_{0}^{a} \Delta B_{z}^{-}(r) r d r+\pi\left(b^{2}-a^{2}\right) \Delta B_{z}^{+} \\
& =-\pi\left(R^{2}-b^{2}\right) \Delta B_{z}^{+},
\end{aligned}
$$

where the conservation of magnetic flux condition (41) was used to eliminate the integral. Since $\Delta \mathrm{B}_{\mathrm{z}}^{+}$is constant, the flux excluded by the beam is then

$$
\Delta \Phi^{a}=\frac{\left(R^{2}-a^{2}\right)}{\left(R^{2}-b^{2}\right)} \Delta \Phi^{b}
$$

from which the beam perpendicular energy per unit length

$$
w_{1}^{a}=B_{0}\left|\Delta \Phi^{a}\right| / 4 \pi
$$

can be determined.

Defining $\varepsilon_{\perp}$ as to average transverse particle energy, Hammer 47 has calculated the magnitude of $\Delta \mathrm{B}_{\mathrm{z}}^{+}$as a function of the beam selfmagnetic field $B_{\theta}(r=R)$ to be

$$
\Delta \mathrm{B}_{z}^{+}=\left(\mathrm{B}_{\theta}(\mathrm{R}) / \mathrm{B}_{z}^{0}\right)\left[\mathrm{B}_{\theta}(\mathrm{R}) / 2\left(\gamma^{2}-1\right)+\varepsilon_{\perp} / \beta \text { e R }\right]
$$

Experimentally, $\Delta B_{z}^{+}, B_{\theta}(R)$, and $B_{0}$ are straightforward to measure but $\gamma=\left(1-\beta^{2}\right)^{-1 / 2}$ is generally not a measurable quantity. However, $\gamma$ can be estimated from the measurement of the net current $I$, utilizing expressions (33) and (36). With respect to the present experiment $\gamma=\left(\gamma_{0} / a_{3}\right)^{1 / 3}$, since we are considering a space charge limited beam. 
In Table VII, expression (42) is compared with compensated diamagnetic 10op measurements from runs D1 through D3. For comparison with the simulation results, the excluded flux $\Delta \Phi$ is in units of $\left(4 \pi n_{b}^{0} m c^{2}\right)^{1 / 2} \lambda_{E^{*}}^{2}$ All loops were located at $z=11.13 \lambda_{E^{*}}$ The radius of each loop is given in column 3. As an example, the loop signal for run D2 is shown in Fig. 16. As a check, two loops were used to measure the excluded flux in run D3. Consistent with expression (43), the .ooop measurements varied by only a geometry factor. The calculated flux change, column 5, is consistently lower than that measured in the simulations, column 4, where we have assumed $\varepsilon_{1} \cong \beta_{0}^{2} Y_{0} \mathrm{mc}^{2} \theta_{\mathrm{s}}^{2}$. The source of this discrepancy is the result of an observable increase in radial motion, resulting from the establishment of equilibrium and passing through the virtual cathode. The radial distribution function for run D2 is shown in Fig. 17. Note the distribution is approximately maxwellian but the 1 /e fold point occurs at $\mathrm{p}_{\mathrm{r}}=1.4-1.5$ rather than $p_{r} \simeq B_{0}^{2} Y_{0} \theta_{s}=1.0$ due to foil scattering. Also in calculating the excluded flux, it was assumed that the average particle pressure $\mathrm{P}_{\theta \theta} \approx \mathrm{P}_{\mathrm{rr}} \approx \frac{1}{2} \mathrm{n} \varepsilon_{\downarrow}$, whereas the actual distribution indicates that $\mathrm{P}_{\theta \theta} \sim 0.5 \mathrm{P}_{\mathrm{rr}} \sim \frac{1}{2} \mathrm{n} \varepsilon_{\perp}$. Thus on the basis of diamagnetic loop measurements, obtaining an accurate estimate of the scattering angle does not appear possible, based upon a simple average pressure model. In column 7 of Table VII, the measured beam transverse energy in $\mathrm{eV} / \mathrm{cm}$ is given. To compare the simulation result with the experimental results, a reduction in pressure due to the decreasing magnetic field strength must be considered. From expression (45), the contribution of the transverse pressure to the diamagnetism is three times larger than 
that due to bearn rotation. Taking this into account, we estimate $W_{1} \cong 0.9-1.1 \times 10^{1.7} \mathrm{eV} / \mathrm{cm}$, which is between the two experimental measurements. Unfortunately, the spread in $W_{1}$ as $\theta_{s}$ goes from zero to thirty degrees is approximately the same as the difference in the two experimental loop measurements. Although we did not simulate the experimental configuration exactly, we are again forced into the conclusion that it may not be possible to obtain reasonably accurate information as to the true beani cemperature on the basis of diamagnetic loop signa1s. 


\begin{tabular}{|c|c|c|c|c|c|}
\hline$\omega_{c} / \omega_{b}^{0}$ & $L^{\left(\omega_{c} / \omega_{b}^{0}\right.}$ & $\Delta_{L}\left(\omega_{c} / \omega_{b}^{0}\right)$ & $a\left(\omega_{c} / \omega_{b}^{0}\right) / R$ & $B_{\theta}^{m}$ & $\mathbf{r}^{\mathrm{m} / \mathrm{R}}$ \\
\hline 1000.0 & 1.75 & 1.000 & 0.500 & $-a .000$ & 0.085 \\
\hline 14.4 & 1.74 & 1.005 & $u .502$ & -0.010 & 0.078 \\
\hline 5.0 & 1.06 & 1.025 & 0.508 & -0.092 & 0.039 \\
\hline 4.0 & 1.05 & 1.040 & 0.515 & -0.130 & 0.043 \\
\hline 3.0 & 1.08 & 1.068 & 0.531 & -0.173 & 0.046 \\
\hline 2.5 & 1.21 & 1.120 & 0.548 & -0.173 & 0.065 \\
\hline 2.0 & 1.51 & 1.170 & 0.566 & -0.153 & 0.085 \\
\hline
\end{tabular}

Table I: Summary of GRADR equilibrium space charge 1imit solutions for a $50 \mathrm{MeV}$ beam with $\mathrm{R} / \mathrm{a}_{0}=2.0$ as a function of external magnetic field strength $\omega_{c} / \omega_{b}^{0}$. The relativistic factor at the center of the beam $\gamma_{L}\left(\omega_{c} / \omega_{b}^{0}\right)$ and the corresponding limiting current $\left.\Delta_{L}(\omega)_{c} / \omega_{b}^{0}\right)$ are shown in columns 2 and 3 . At the space charge limit, the equilibrium beam radius $a\left(\omega_{c} / \omega_{b}^{0}\right)$, the maximum azimuthal velocity $\beta_{\theta}^{m}$, and its corresponding position $r^{\mathbb{m}}$ are given in columns 4 through 6 , respectively. Note that $\Delta_{L}\left(\omega_{c} / \omega_{b}^{0}\right)$ is given relative to $\Delta_{L}(\infty)$. 


\begin{tabular}{|c|c|c|c|c|c|c|}
\hline Run & $\Delta_{\mathrm{I}}^{\mathrm{sin}}$ & $\Delta_{\mathrm{T}}^{\text {sim }}$ & $-e \phi^{c}(r=0) / m c^{2}$ & $-e \phi^{\mathrm{T}}(r=0) / \mathrm{mc}^{2}$ & $\gamma_{e}$ & $\lambda / a_{0}$ \\
\hline Al & 0.5 & 0.5 & $1.1-1.2$ & $1.1-1.2$ & 4.21 & 4.67 \\
\hline $\mathrm{A} 2$ & 0.7 & 0.7 & $1.6-1.7$ & $1.6-1.7$ & 4.01 & 3.76 \\
\hline A3 & 0.9 & 0.9 & $2.1-2.3$ & $2.1-2.3$ & 3.72 & 3.07 \\
\hline A4 & 1.0 & 1.0 & $2.4-2.6$ & $2.4-2.6$ & 3.57 & 2.80 \\
\hline A5 & 1.1 & 1.10 & $2.7-2.9$ & $2.7-2.9$ & 3.41 & 2.55 \\
\hline $\mathrm{A} 6$ & 1.3 & 1.30 & $3.3-3.5$ & $3.0-3.2$ & 2.96 & 2.03 \\
\hline A7 & 1.5 & 1.35 & $3.5-4.3$ & $3.0-3.4$ & - & - \\
\hline A8 & 1.7 & 1.37 & $3.3-4.5$ & $3.0-3.6$ & - & - \\
\hline $\mathrm{A} 9$ & 2.0 & 1.39 & $3.3-4.5$ & $2.8-3.4$ & - & - \\
\hline A10 & 2.5 & 1.45 & $3.3-4.5$ & $3.0-3.5$ & - & - \\
\hline All & 3.0 & 1.40 & $3.3-4.5$ & $2.9-3.7$ & - & - \\
\hline
\end{tabular}

Table II: Summary of simulation results for fixed magnetic field strength $\omega_{c} / \omega_{b}^{0}=4.0$, with $\gamma_{0}=5.0$ and $R / a_{0}=2.0$. Columns 2 and 3 are the injected and transmitted currents. The potential on axis associated with the virtual cathode is given in column 4; and column 5 is the potential on axis of the transmitted beam. Columns 6 and 7 are the relativistic factor of electrons on the beam edge calculated by GRADR, and the wavelength of surface modulation given by (23). 


\begin{tabular}{|c|c|c|c|c|}
\hline Run & $\omega_{c} / \omega_{b}^{0}$ & $\Delta_{\mathrm{T}}$ & $-e \phi^{c}(r=0) / m c^{2}$ & $-e \phi^{\mathrm{T}}(r=0) / m c^{2}$ \\
\hline $\mathrm{B} 1$ & 5.0 & 0.292 & none & 1.58 \\
\hline $\mathrm{B} 2$ & 5.0 & 0.249 & 2.00 & 1.79 \\
\hline B3 & 5.0 & 0.248 & 2.04 & 1.77 \\
\hline B4 & 2.75 & 0.268 & 1.91 & 1.74 \\
\hline B5 & 2.00 & 0.283 & 1.83 & 1.76 \\
\hline $\mathrm{B} 6$ & 1.50 & 0.288 & 1.80 & 1.77 \\
\hline B7 & 1.00 & 0.290 & none & 1.74 \\
\hline B8 & 0.50 & $0.26-0.27$ & none & $1.30-1.70$ \\
\hline B9 & 0.25 & $0.19-0.20$ & none & hits wall \\
\hline B10 & 0.00 & 0.0 & none & hits wa11 \\
\hline
\end{tabular}

Table IIL: Summary of simulations for fixed injection current $\Delta_{I}=0.292$, and various values of magnetic field strength $\omega_{c} / \omega_{b}^{0}$ given in column 2 . Run $B 1$ is a test in which the injection plane is not grounded. The transmitted current is given in column 3, with the potential of the virtual cathode on axis in column 4. The potential on axis associated with the transmitted beam is given in column 5 . 


\begin{tabular}{|c|c|c|c|c|c|c|}
\hline$\omega_{c} / \omega_{b}^{0}$ & $\gamma_{0}$ & $R / a_{0}$ & $L / a_{0}$ & $\Delta_{I}$ & $\Delta_{L}(5.0,0.0)$ & $\Delta_{L}^{\text {sin }}$ \\
\hline 5.0 & 2.46 & 2.08 & 3.33 & 3.40 & 0.432 & 0.490 \\
\hline
\end{tabular}

Table IV: Summary of a simulation for which the injection current $\Delta_{I}$ great1y exceeds the theoretical space charge limiting current $\dot{\Delta}_{\mathrm{L}}(5.0,0.0)$. The basic parameters are given in columns 1 through 4 , where the beam radius $a_{0} \omega_{b}^{0} / c=3.69$. The annular transmitted current measured at $z=0.91 \mathrm{~L}$ Is given in column 7 . 


\begin{tabular}{|c|c|c|c|c|c|c|}
\hline Run & $\omega_{c} / \omega_{b}^{0}$ & $\underline{\theta_{s}}$ & $\phi^{c}(r=0) / m c^{2}$ & $z_{\phi} / \lambda_{E}$ & $\Delta_{\mathrm{T}}\left(\omega_{\mathrm{c}} / \omega_{\mathrm{b}}^{0}, \theta_{\mathrm{s}}\right)$ & $\mathcal{L}$ \\
\hline D1 & 5.0 & 0 & 2.04 & 3.20 & 0.248 & 4.42 \\
\hline D2 & 5.0 & 20 & 1.75 & 3.19 & 0.199 & 4.70 \\
\hline D3 & 5.0 & 30 & 1.43 & 3.60 & 0.165 & 5.38 \\
\hline D4 & 2.75 & 20 & 1.70 & 3.66 & 0.203 & 4.19 \\
\hline D5 & 2.00 & 20 & 1.74 & 5.01 & 0.210 & 4.00 \\
\hline D6 & 1.00 & 20 & 1.45 & 12.3 & 0.273 & 2.93 \\
\hline D7 & 0.50 & 20 & Hit Wall & Hit Nal1 & 0.270 & 1.11 \\
\hline $\mathrm{D} 8$ & 0.25 & 20 & Hit Wall & Hit Wa11 & 0.277 & 1.02 \\
\hline
\end{tabular}

Table V: Sumnary of simulations for a scattered beam. The basic parameters are $\gamma_{0}=3.0, \mathrm{R} / \mathrm{a}_{0}=7.39$, and $\mathrm{L} / \mathrm{a}_{0}=22.20$. Columns 2 and 3 are the magnetic field strength and scattering angle $\theta_{s}$. The maximum potential of the virtual cathode on axis and its axial position are given in columns 4 and 5 . For each run the transmitted current appears in column 6 . The injected current was $\Delta_{I}=0.275$. In units of $\mu_{0} / 4 \pi=10^{-7} \mathrm{H} / \mathrm{m}$, the inductance $\mathcal{L}$ measured at $z=11.13 \lambda_{E}$ is given in column 7 . 


\begin{tabular}{l|l|l}
$x(\mu \mathrm{m})$ & $F(\mathrm{Al})$ & $F(\mathrm{~T} i)$ \\
\hline 12.7 & 0.054 & 0.168 \\
25.4 & 0.132 & 0.395 \\
20.8 & 0.310 & 0.913 \\
76.2 & 0.501 & 1.47
\end{tabular}

Table VI: Foil scattering function $F$ for $A I$ and $T i$ foils of thickness $x(\mu m)$. 


\begin{tabular}{|c|c|c|c|c|c|c|}
\hline Run & $\theta_{\mathrm{S}}$ & $\mathrm{b} / \lambda_{\mathrm{E}}$ & $\Delta \bar{\Phi}_{\mathrm{sim}}^{\mathrm{b}}$ & $\Delta \tilde{\Phi}_{\mathrm{ca}}^{\mathrm{b}}$ & $\Delta \tilde{\Phi}_{\operatorname{sim}}^{\mathrm{a}}$ & $\mathrm{W}_{1}(\mathrm{eV} / \mathrm{cm})$ \\
\hline D1 & 0 & 1.53 & -0.107 & -0.067 & -0.100 & 0.71 \\
D2 & 20 & 1.53 & -0.224 & -0.150 & -0.211 & 1.60 \\
D3 & 30 & 2.97 & -0.257 & -0.208 & -0.290 & 2.06 \\
D3 & 30 & 5.58 & -0.163 & -0.130 & -0.294 & 2.09 \\
\hline
\end{tabular}

Table VII: Summary of diamagnetic loop measurements.

Columns 4 and 5 are the excluded flux measurements obtained from simulation $\Delta \tilde{\Phi}_{\text {sim }}^{\mathrm{b}}$ and from expression (42) $\Delta \tilde{\Phi}_{\text {cal }}^{\mathrm{b}}$, respectively. 


\section{REFERENCES}

1) M. L. Sloan and W. E. Jrummond, Phys. Rev. Lett. 31, 1234 (1973).

2) P. Sprangle, A. T. Drobot, and W. M. Manheimer, Phys. Rev. Lett. 36, 1180 (1976).

3) B. B. Godfrey, R. J. Faehl, B. S. Newberger, W. R. Shananan, and L. E. Thode, in the proceedings of the 2nd International Topical Conference on High Power Electron and Ion Beam Research and Technology, Oct. 1977, Cornell University, Ithaca, New York, paper E7.

4) V. L. Granatstein, M. Herndon, P. Sprangle, Y. Carmel, and J. A. Nation, Plasma Phys. 17, 23 (1975).

5) V. L. Granastein, P. Sprangle, M. Herndon, R. K. Parker, and S. P. Schlesinger, J. App1。 Phys. 46, 3800 (1975).

6) E. Ott and W. M. Manheimer, IEEE Trans. P1as, Sci, PS-3, J. (1975).

7) P. Sprangle, R. K. Parker, J. Pasour, M. Herndon, S. D. Schlesinger, and J. L. Seftor, Phys. Rev. A 14, 1194 (1976).

8) M. Friedman, Phys. Rev. Lett. 31, 1107 (1973).

9) I. E. Thode, "Preliminary Investigation of Anomalous Relativistic Electron Beam Deposition Into Dense $\left(10^{17}\right.$ to $\left.10^{20} \mathrm{~cm}^{-3}\right)$ P1asma," LA-UR-77-102 (1977).

10) L. E. Thode, "Effect of Electron-Ion Collisions on the Nonlinear State of the Relativistic Two-Stream Instability," LA-UR-77-1437 (1977).

11) A. I. Pavlovskii, V. S. Bosamykin, G. D. Kuleshov, A. I. Gerasimov, V. A. Tananakin, and A. P. Klement'ev, Dokl. Akad. Nauk SSSR 222, 817 (1975) [Sov. Phys. Dokl. 20, 441 (1975)]. 
12) V. I. Kazacha and I. V. Kozhukhov, Zh. Tech. Fiz. 46, 1477 (1976) [Sov. Phys. Tech. Phys. 21, 841 (1976)].

13) B. Ecker and S. Putnam, IEEE Trans. Nuc1. Sci. NS-20, 301 (1973).

14) G. W. Kuswa, L. P. Bradley, and G. Yonas, IEEE Trans. Nucl. Sci. NS-20, 305 (1973).

15) D. C. Straw and R. B. Miller, Appl. Phys. Lett. 25, 379 (1974).

16) C. L. Olson, Phys. Fluids 18, 585; 598 (1975).

17) C. A. Kapetanakos (Private communication).

18) R. C. Davidson, Theory of Nonneutral Plasmas (Benjamin, New York, 1974).

19) H. V. Wong, M. L. Sloan, J. R. Thompson, and A. T. Drobot, Phys. Fluids 16, 902 (1973).

20) L. S. Bogdankevich and A. A. Rukhadze, Usp. Fiz. Nauk. 103, 609 (i971) [Sov. Phys. - Usp. 14, 163 (1971)].

21) J. R. Thompson and M. L. Sloan, in the proceedings of the 2 nd International Topical Conference on High Power Electron and Ion Beam Research and Technology, Oct. 1977, Cornell University, Ithaca, New York.

22) T. Genoni and W. Proctor (Private communication).

23) C. L. 01son and J. W. Poukey, Phys. Rev. A9, 263 (1974).

24) C. K. Birdsall and W. B. B.ridges, J. Appl. Phys. 32, 2611 (1961); 34, 2946 (1963).

25) D. A. Dunn and I. T. Ho, AIAÁ J. 1, 2770 (1963).

26) P. C. deJagher, H. J. Hopmen, and B. Jurgens, Proc. 12th International Conference Phenomena in Ionized Gases, 1975, p. 342. 
27) V. S. Voronin, Yu. T. Zozulya, and A. N. Lebedev, Zh. Tech. Fiz. 42, 546 (1972) [Sov. Phys. Tech. Phys. 17, 432 (1972)].

28) J. W. Poukey and N. Rostoker, Plasma Phys. 13, 897 (1971).

29) M. Reiser, Phys. Fluids 20, 477 (1977).

30) G. P. Mkheidze, V. I. Pulin, M. D. Raizer, and L. E. Tscpp, Zh. Eksp. Teor. Fiz. 63, 104 (1972) [Sov. Phys - JETP 36, 54 (1973)].

31) M. Friedman, App1. Phys, Letters 24, 303 (1974).

32) J. A. Nation and M. Read, Appl. Phys, Lett. 23, 426 (1973).

33) M. E. Read and J. A. Nation, J. Plasma Phys. 13, 127 (1975).

34) Yu. I. Abrashitov, V. S. Koidan, V. V. Konyukhov, V. M. Lagunov, V. N. Luk'yanov, K. L. Mekler, and D. D. Ryutov, Zh. Eksp. Teor. Fiz. 66, 1324 (1974) [Sov. Phys. - JETP 39, 647 (1974)].

35) R. B. Miller and D. C. Straw, J. Appl. Phys. 48, 1061 (1977).

36) A. A. Kolomensky, E. G. Krastelev, A. N. Lebedev, V. S. Voronin, B.N. Yablokov, P. N. Lebedev, and B. N. Yablokov, in the proceelings of the 2nd International Topical Conference cn High Power Electron and Ion Beam Research and Technology, Oct. 1977, Cornell University, Ithaca, New York.

37) B. B. Godfrey, J. Comp. Phys. 19, 58 (1975).

38) B. B. Godfrey, J. Comp. Phys. 15, 504 (1974).

39) B. B. Godfrey, IEEE Plas. Sci., to be published, and references therein.

40) B. B. Godfrey, submitted to IEEE Plas. Sci.

41) P. J. Davis and I. Polonsky in Handbook of Mathematical Functions, edited by M. Abramowitz and I. A. Stegun, National Bureau of Standards Applied Mathenatics Series No. 55 (U. S. Government Printing office, Washington, D. C. 1964), P. 897. 
42) J. F. Traub, Iterative Methods for the Solution of Equations (Prentice-Ha11, Englewood Cliffs, 1964), Ch. 10.

43) R. C. Davidson and N. A. Kra11, Phys. Fluids 13, 1343 (1970).

44) D. Prono, B. Ecker, N. Bergstrom, and J. Benford, Pinys. Rev. Lett. 35, 438 (1975).

45) L. E. Thode, Phys. Fluids 19, 831 (1976).

46) H. A. Bethe, Phys. Rev. 89, 1256 (1953).

47) D. A. Hammer, Phys. Fluids 17, 1260 (1974). 


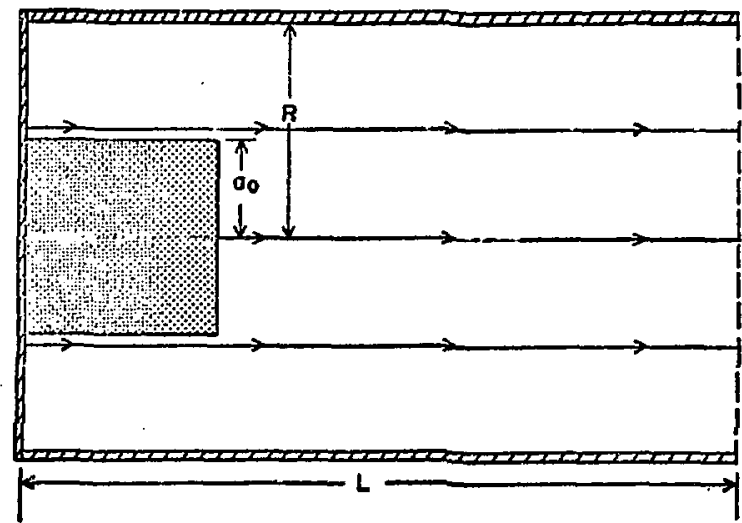

Ffg. 1. Basic spatial configuration for all CCUBE beam propagation sirulations.

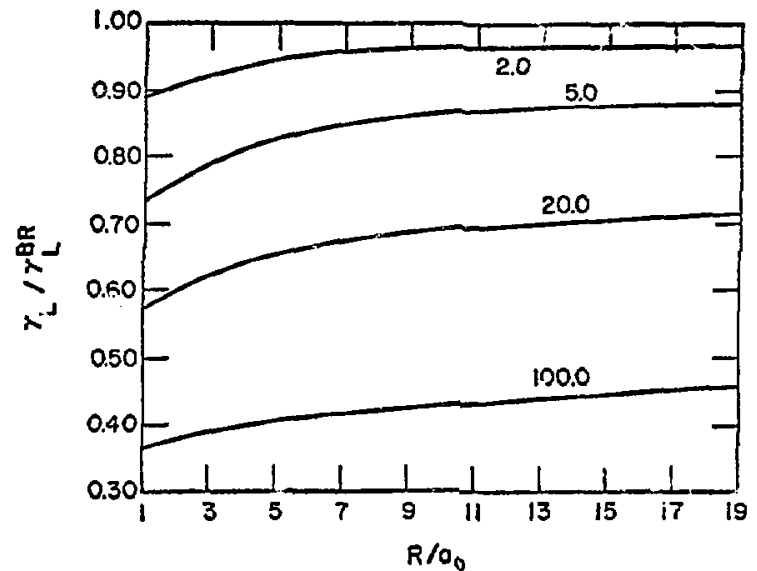

Fig. 3. Beam relativistic factor on axis at the space charge limit as a function of $\mathrm{R} / \mathrm{a}_{0}$ for various values of $\gamma_{0}$, as determined numerically by GRADR.
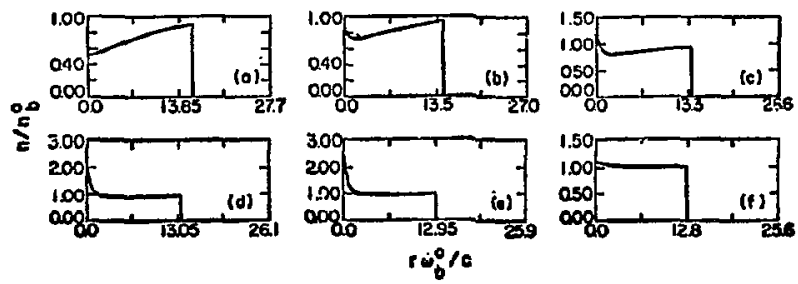

Fig. 5. Self-consistent radial density as a function of magnetic field strength: a) $\left.\left.\omega_{c} / \omega_{b}=2.0, b\right) \omega_{c} / \omega_{b}=2.5, c\right) \omega_{c} / \omega_{b}=3.0$, d) $\left.\omega_{c} / \omega_{b}^{0}=4.0, e\right) \omega_{c} / \omega_{b}^{0}=5.0$, and f) $\omega_{c} / \omega_{b}=14.4$. Solution is for a $50 \mathrm{MeV}$ beam with $R / a_{0}=2.0$. as calculated by GRADR.

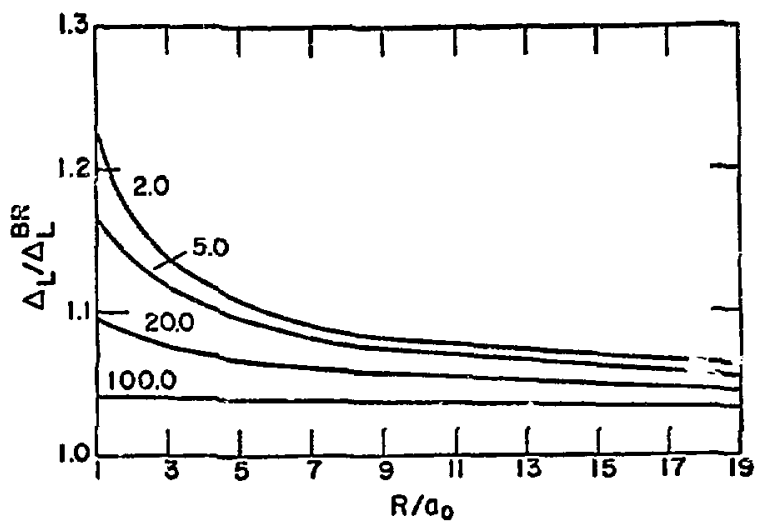

Fig. 2. Space charge limiting current $\Delta_{\mathrm{L}} / \Delta^{B R}$ as a function of $\mathrm{R} / \mathrm{a}_{0}$ for various values of $\Upsilon_{0}$, as determined numerically by GRADR.

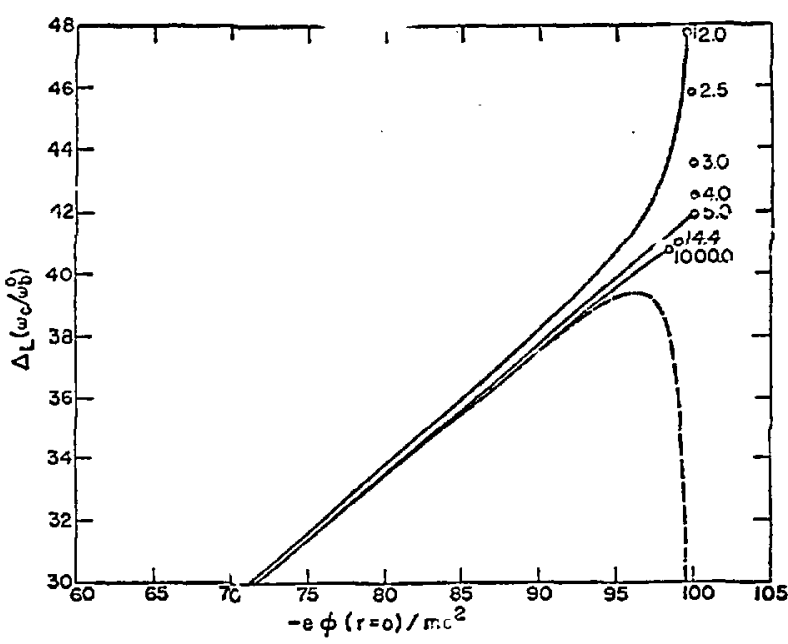

Fig. 4. Equilibrium current as a functicn of the radial potential on axis for a $50 \mathrm{MeV}$ beam with $R / a_{0}=2.0$. The dashed line is $\triangle^{\mathrm{BR}}$. Solid lines are solutions determined by GRADR. The space charge Iimiting current (•) as a function of $\omega_{c} / \omega_{b}^{0}$ is also
shown. 

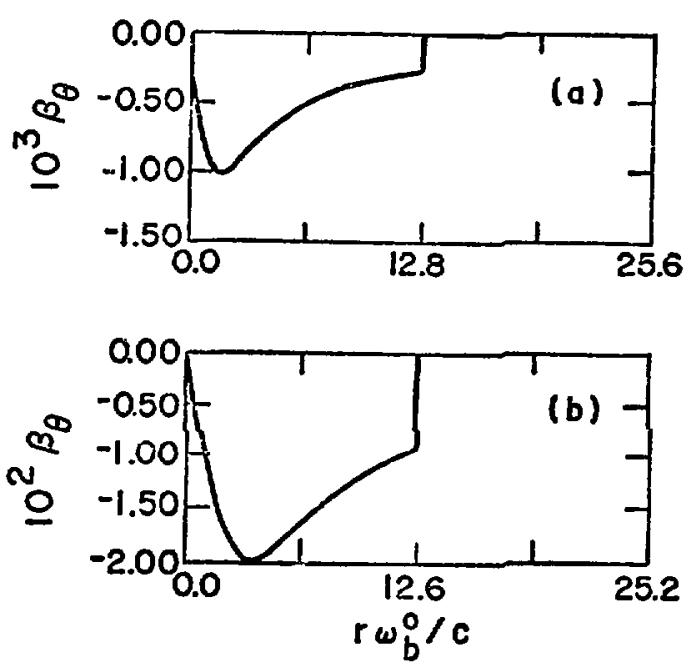

Fig. 6. Self-consistent azimuthal precessional velocity $B_{9}$ as a function of $r$ for a) $\Delta=0.95 \Delta_{\mathrm{L}}$ and b) $\Delta=\Delta_{\mathrm{L}}$. The magnetic field strength is $\omega_{c} / \omega_{f}=14.4$. Solution is for a $50 \mathrm{MeV}$ beam with $\mathrm{R} / \mathrm{a}_{0}=$ 2.0, as calculated by GRADR. Note that $\beta_{\theta}^{m}$ occurs at $r^{m} \ll a_{0}$ as indicated by (19) and $(20)$.

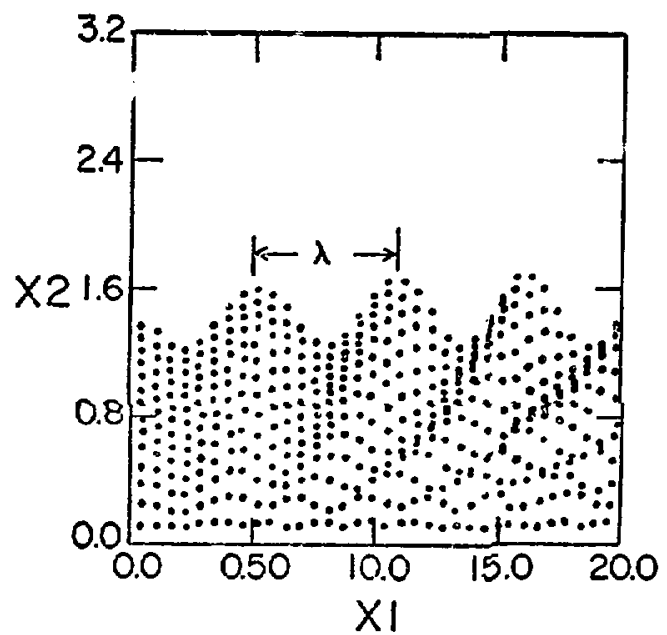

Fig. 8. Farticle configuration space for run $A 4$ at $\tau=76.09$. Wavelength of the stationary wave is given by (23) and modulation amplitude by (27). Note that the self-electric field is shorted near the anode foil.

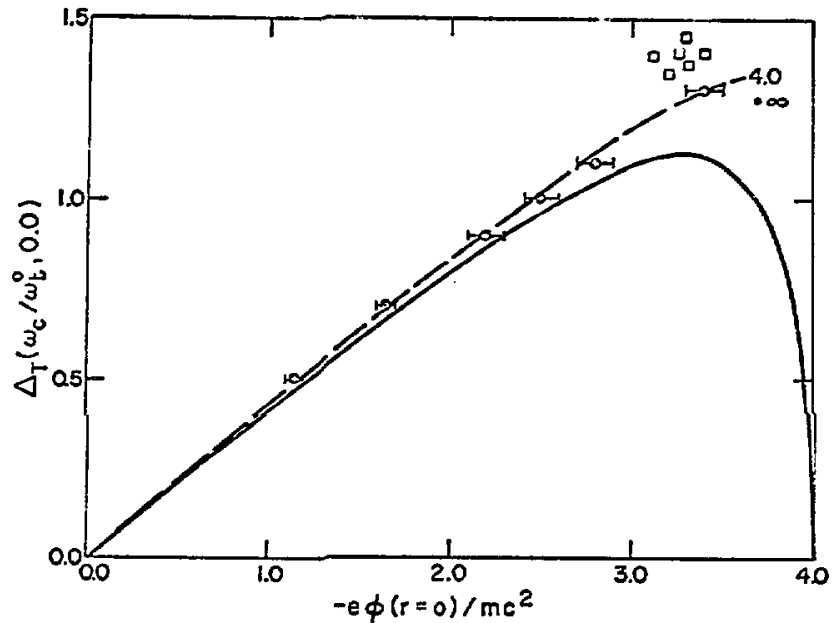

Fig. 7. Transmitted current $\Delta \mathbf{T}$ as a function of the radial potential on axis for $Y_{0}=5.0$ and $\mathrm{R} / \mathrm{a}_{0}=2.0$. The solid line is $\Delta^{\mathrm{BR}}$ given by (17). The GRADR solution for $\omega_{c} / \omega_{b}=4.0$ is the dashed line. Simulation results for $\Delta_{I}<\Delta_{L}\left(\omega_{c} / \omega_{b}\right)$ are denoted by the solid dots, whereas the results for $L_{I}>\Delta_{L}\left(\omega_{c} / \omega_{b}^{0}\right)$ are depicted by open squares.
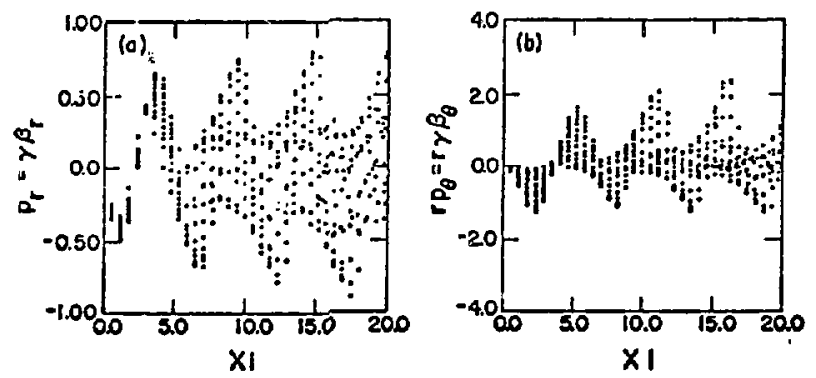

Fig. 9. a) Radial momentum $p_{r}=\gamma \beta_{r}$ as a function of XI for run A4 at $\tau=76.04$, b) Azimuthal momentum $r p_{\theta}=r \gamma B_{\theta}$ as a function of $x 1$ for run $A 4$ at $T=76.04$. Basic spatial dependence is given by (29). 


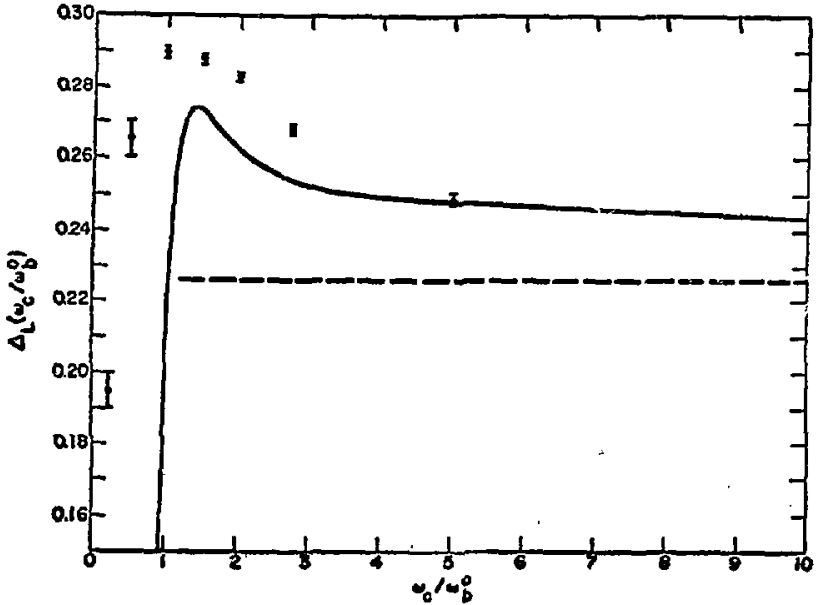

Fig. 10. Transmitted current $\Delta_{T}$ as a function of magnetic field strength $\omega_{c} / \omega f$, for $\gamma_{0}=3.0$ and $\mathrm{R} / \mathrm{a}_{0}=7.39$. The solid line is the GRADR solution. For comparison, $\triangle^{B R}$ is the dashed line. Simulation results, runs $\mathrm{Bl}$ through $\mathrm{B} 10$, are depicted by solid dots.

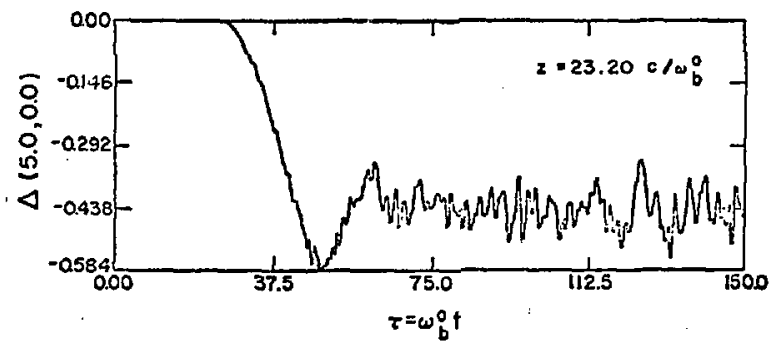

Fig. 12. Transmitted current as a function of time for $\Delta_{T}=3.40$. Basic parameters are $\gamma_{0}=2.46, R / a_{0}=2.08, L / a_{0}=3.33$, ard $\omega_{c} / \omega_{b}^{0}=5.0$.

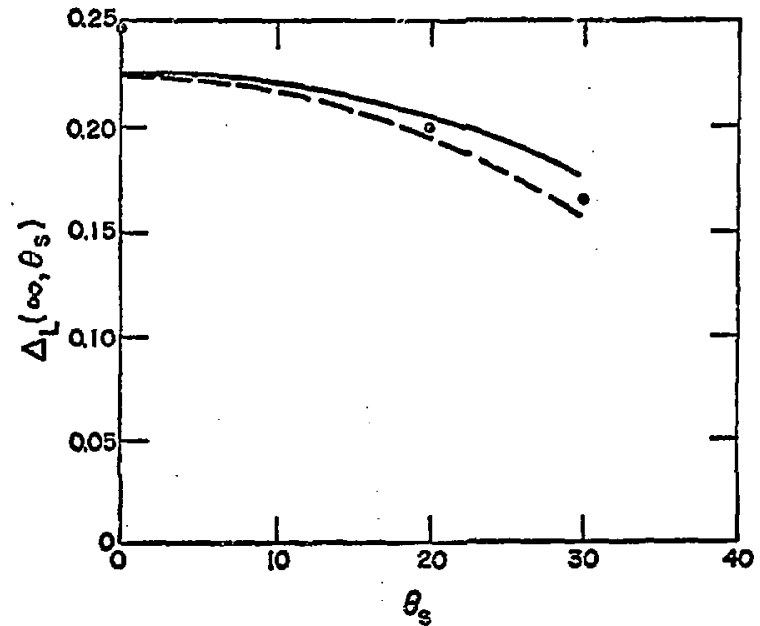

Fig. 14. Space charge limiting current as a function of scattering angle. Dashed line is $\Delta_{\mathrm{T}}^{M}\left(\infty, \theta_{s}\right)$ and solid line is $\Delta_{\mathrm{L}}\left(\infty, \theta_{s}\right)$. Simulation results, runs D1 through D3, are depicted by solid dots.
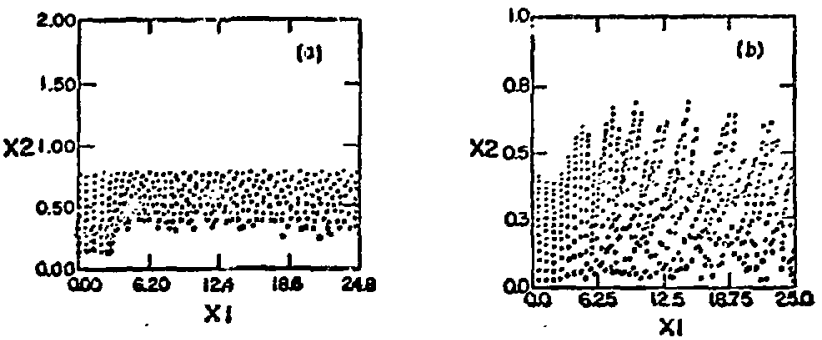

Fig. 11. a) Particle configuration space for run $B 3$ with $\omega_{c} \omega_{b}=5.0$. b) Particle configration space for run $\mathrm{BJ}$ with $\omega_{c} / \omega_{b}^{0}=2.0$.

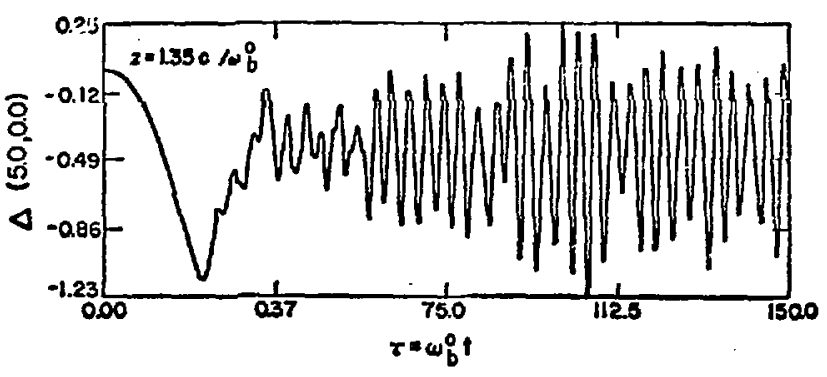

Fig. 13. Net current measured by a loop located between the anode foil and the virtual cathode as a function of time. Basic parameters are the same as in Fig. 12.

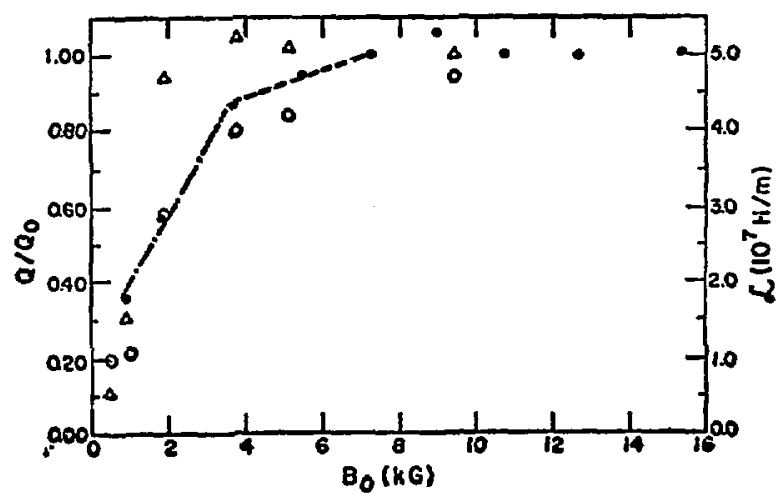

Fig. 15. Simulation ( $\triangle$ ) and experimental (') calorimeter results as a function of magnetic field strength. Beam inductance per unft length (o) via simulation is also shown. 


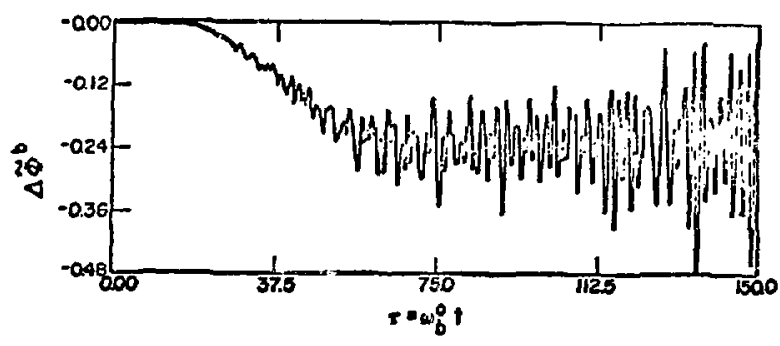

Fig. 16. Diamagnetic loop signal as a function of time, run $\mathrm{D} 2$.

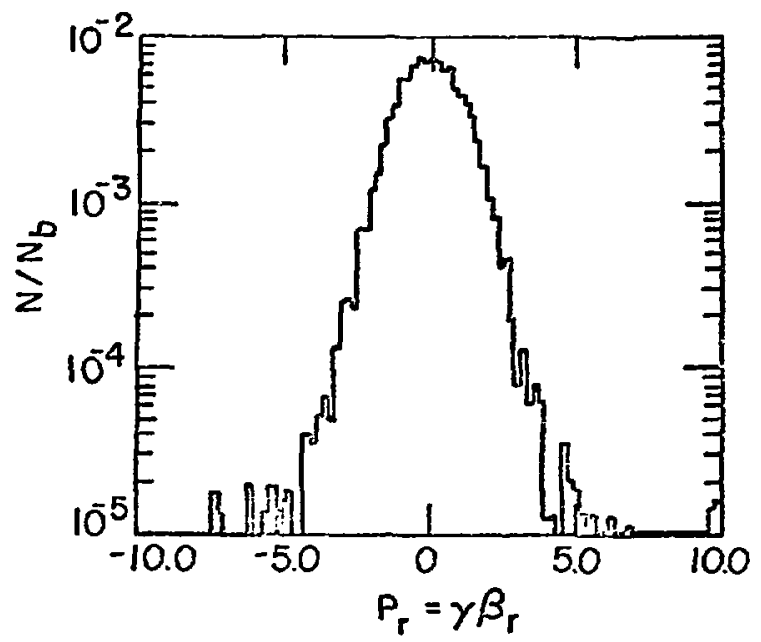

Fig. 17. Radial momentum $p_{r}=\gamma \beta_{r}$ distribution function for run D2 at $\tau=149.94$. 
[Reprinted from Physical Review A 16, 377-379 $(1977)$.

APPENDIX D

INSTABILITY OF A NONNEUTRAL ELECTRON BEAM PROPAGATING IN AN INHOMOGENEOUS MAGNETIC FIELD 


\title{
Instability of a nonneutral electron beam propagating in an inhomogeneous magnetic field
}

\author{
William R. Shanahan \\ Theoretical Division. Los Alamos Scientific Laboratory. Los Alamos. New Mexico 87545
}

(Recieived 16 December 1976)

\begin{abstract}
The stability of a nonneutral, rapidly rotating electron beam propagating in an axially inhornogeneous magnetic field is considered. It is shown that there exists an instability, driven by ar axial shear in the rotation velocity, which does not exist in the uniform limit. Selected numerical results are presented.
\end{abstract}

Numerous studies exist of the equilibria and stability of a nonneutral electron beam propagating in a uniform magnetic field.' No similar examination seems yet to have been made for the case of a inhomogeneous field. In this paper we demonstrate the important conclusion that the proper inclusion of the effects of such an inhomogeneity can reveal the existence of an instability. This instability has no analog in the limit of vanishing zero-order gradient.

The problem considered is that of a nonneutral rapidly rotating electron beam propagating in the direction of a decreasing magnetic field. The equilibrium is assumed to be such that the beam properties are at each point identical to those of a rigid-rotor corresponding to the local value of the applied magnetic field. The radial components of both the magnetic field and the zero-order beam velocity are neglected. From $\nabla \cdot \overrightarrow{\mathrm{B}}^{0}=0$, we find the est imate $\left|B_{r}^{0}\right| \cong(r / L) B_{\varepsilon}^{0}$, where $L$ is a gradient scale length. Since $v_{r}^{0} / v_{s}^{0} \sim B_{r}^{0} / B_{s}$, we see that our assumptions are justified, provided that the radial extent of the beam is small compared to $L$. From the imnediately preceding approximate equality, we may also derive that

$$
\frac{1}{n^{0}} \frac{d n^{0}}{d z}=\frac{1}{B^{0}} \frac{d B^{0}}{d z} \text {. }
$$

Consequently, the axial derivative of the beam density will be small compared to that of the magnetic field provided that the condition for beam equilibrium is well satisfied. Since this shall aiways be assumed to be the case, we shall neglect the density variation also.

The important point to be noted concerning the equilibrium describe $t$ above is that spatial variation of the magnetic eld induces an axial shear in the local frequency of rigid rotation,

$$
\omega_{R}(z)=\frac{\Omega(z)}{2}\left[1+\left(1-\frac{2 \omega^{2}}{\Omega(z)^{2}}\right)^{1 / 2}\right] \text {. }
$$

This shear can drive an instability. Note that this instability has no analog in the case of a uniform magnetic field. Consequently, its existenc: is not revealed through a treatment based on the insatz $k_{\varepsilon}-i d / d z$, but only through the analysis to be presented below.

The present problem was suggested by considerations relating to the stability of a device which has been proposed for the purpose of collectively accelerating ions. ${ }^{2}$ However, the electron beam equilibria which are teing considered for this particular application are assumed to be in the slow mode of rotation. In this mode the effects of rotation are of order $n^{0} / B^{\circ}$. By the above ordering, we have neglected the effects of such terms. Hence the considerations of this paper are not immediately relevant to this device. We are nevertheless presenting the following calculations since, on the one hand, schemes for collectively accelerating ions which do employ rapidly rotating electron beams propagating in an inhomogeneous magnetic field have already been proposed, ${ }^{3}$ and, on the other, it seems likely that applications of electron beams propagating in complex field geometries will be subjected to increasing study. It should also be emphasized that the evaluation of the effects of the instability considered here on particular devices will generally involve the inclusion of relativistic, electromagnetic, and surface effects. Although such effects are probably of importance for particular applications, they are clearly not crucial for the existence of the instability. Therefore, they are not included here. We concentrate instead on the effects of magnetic field inhomogeneity.

The basic equations to be employed are the fluid equations of motion, expressed in cylindrical coordinates, from which the time and aziumthal dependencies have been removed by Fourier analysis. These may be expressed as

$$
\begin{array}{r}
\left(-i \omega+i l \omega_{R}(z) \pm i\left[2 \omega_{R}(z)-\Omega(z)\right]+v_{\alpha}^{0} \frac{d}{d z}\right) v^{ \pm(1)} \\
=\frac{e}{m} \frac{d \phi^{(1)}}{d r} \mp \frac{e}{m} \frac{l \phi^{(1)}}{r} \mp \frac{i d \omega_{R}(z)}{d z} v_{k}^{(2)}, \\
\left(-i \omega+i l \omega_{R}(z)+v_{\alpha}^{0} \frac{d}{d z}\right) v_{R}^{(1)}=\frac{e}{m} \frac{d \phi^{(1)}}{d z},
\end{array}
$$

where $v^{ \pm(1)}=v_{r}^{(1)} \pm i \varepsilon_{\theta}^{(1)}$, and the other symbols em- 
ployed have their usual meanings. Equations (1) and (2) may be formally solved for $v^{t(1)}$ through the introduction of the integrating factors $\exp \left(\int^{x} \alpha_{z} d z^{\prime}\right)$, where

$$
\alpha^{*}(z)=\left\{-\omega+l \omega_{R}(z) \pm\left[2 \omega_{R}(z)-\Omega(z)\right]\right\} / v_{s}^{0} .
$$

We may then eliminate $v^{ \pm(1)}$ from the equation of continuity and Poisson's equation to yield a second equation in $v_{2}^{(1)}$ and $\phi^{(1)}$ to supplement Eq. (2).
It is at this point convenient to eliminate the radial dependence of all quantities through a Bessel transform, so that we have for all quantities $\psi$,

$$
\begin{aligned}
& \psi(r, \theta, z, \ell)=\sum_{i=0}^{\infty} \int_{0}^{\pi}\left(\omega, k_{1}, l, z\right) e^{-i \omega t+12 \theta} \\
& \times J_{8}\left(k_{1} r\right) k_{1} d k_{1} d \omega .
\end{aligned}
$$

Upon so doing, snd dropping the tilde on transforms, there results

$$
\begin{aligned}
& \left(-i \omega+i l \omega_{\Omega}(z)+v_{*}^{0} \frac{d}{d z}\right)\left(-k_{1}^{2}+\frac{d^{2}}{d z^{2}}\right) \phi^{(1)} \\
& =\frac{4 \pi e}{2 v_{\alpha}^{0}} \sum_{\sigma=z} \exp \left(-\int \alpha_{0} d z^{\prime}\right) \int^{4} \exp \left(\int^{z^{\prime}} \alpha_{0} d z\right)\left(\frac{e}{m} k_{1}^{2} \phi\left(z^{\prime}\right)-2 \sigma l v_{\varepsilon}^{(1)} \frac{d \omega_{R}\left(z^{\prime}\right)}{d z^{\prime}}\right) d z^{\prime}-4 \pi e \frac{d v_{c}^{(1)}}{d z} .
\end{aligned}
$$

Equations (2) and (3) are the desired equations, and are exact within the limits of our assumptions regarding the equilibrium. In the case of a uniform magnetic field these equations may of course be Fourier transformed in $z$ as well to yield the well-known dispersion relation for electrostatic waves propagating along a nonneutral, magnetized plasma column:

$$
k_{s}^{2}+k_{\perp}^{2}=\frac{\omega_{R}^{2} k_{R}^{2}}{\left(\omega-l \omega_{R}-k_{\alpha} v_{s}^{0}\right)^{2}}+\frac{\omega_{\rho}^{2} k_{1}^{2}}{\left(\omega-l \omega_{R}-k_{\varepsilon} v_{g}^{0}\right)^{2}-\left(\Omega-2 \omega_{R}\right)^{2}} .
$$

The solutions of (4) reflect the stability of a rigid-rotor equilibrium in a uniform magnetic field against electrostatic perturbations.

The effects of a weak axial inhomogeneity may be included by making for $\phi$ the usual WKB substitution

$$
\phi(z)=\bar{\phi}(z) \exp i \int^{z} k_{z}\left(z^{\prime}\right) d z^{\prime},
$$

where the second and higher-order derivatives of $\tilde{\phi}$ are to be neglected, and $k_{z}(z)$ is to be determined from the local version of (4). Making this substitution, expanding all quantities up to terms involving first derivatives, and neglecting terms involving products of two or more derivatives, we note that the integrals in (3) can be computed explicity. Eliminating the axial velocity perturbation, there results

$$
\begin{aligned}
& A \frac{d \dot{\phi}}{d z}=(B+C) \dot{\phi}
\end{aligned}
$$

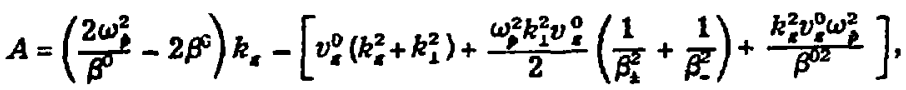

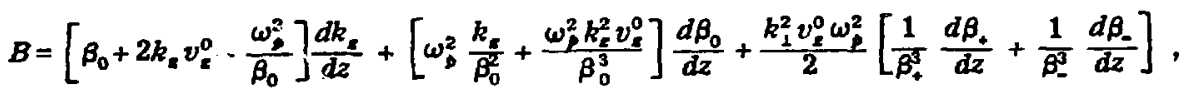

$$
\begin{aligned}
& C=\frac{l}{2} \frac{k_{s}}{\beta_{0}}\left[\frac{1}{\beta_{+}}+\frac{1}{\beta_{-}}\right] \frac{d \omega_{R}(z)}{d z}, \beta_{t}=v_{z}^{0}\left(\alpha^{*}+k_{\varepsilon}\right), \quad \beta_{0}=k_{s}(z) v_{s}^{0}+l \omega_{R}(z)-\omega .
\end{aligned}
$$

Equation (5) may be somewhat simplified by factoring $\bar{\phi}=\bar{\phi}_{v} \tilde{\phi}_{a}$, where $\bar{\phi}_{x}$ will contain the variation arising from the spatial growth induced by the instability, while $\phi_{\varepsilon}$ reflects the change in amplitude arising from the inhomogeneity. An equation for the latter may be found by noting that in the absence of a source of free energy, the amplitude must vary in such a manne $r$ that wave energy is conserved. The computation is very similar to those long employed in plasma theory, ${ }^{4}$ although it is here somewhat more involved, since the small signal theory for a rotating electron beam cannot be expressed in a strictly conservative form. ${ }^{5}$ The final equation for the quantity of interest $\bar{\phi}_{u}$ is

$$
\begin{aligned}
& \frac{d \bar{\phi}_{z}}{d z}=\frac{C}{A} \bar{\phi}_{u}=-l \omega_{p}^{2} \beta^{0^{2}} \beta^{*} \beta k_{z} \frac{d \omega_{R}(z)}{d z} \phi_{u}\left\{\left(\beta^{+} \beta\right)^{2}\left[v_{z}^{0}\left(\beta^{0^{2}} k_{\perp}^{2}+\beta^{0^{2}} k_{z}^{2}+\omega_{p}^{2} k_{q}^{2}\right)+2 k_{z} \beta^{09}-2 k_{z} \omega_{p}^{2} \beta^{\circ}\right]\right. \\
& \left.+v_{s}^{0} k_{\perp}^{2} \omega_{\beta}^{2} \beta^{0^{2}}\left(\beta^{\star 2}+\beta^{2}\right)\right\}^{-2} \text {, }
\end{aligned}
$$


showing clearly that the spatial gain is proportional to the axial shear of the rotational velocity.

An evaluation of the spatial growth rate generally involves numerical computation since one must know $k_{z}(z)$ as a function both of frequency and local magnetic field, a dependence which follows from (4), a sixth-order equation in $k_{a}(z)$. However, for $k_{1} / k_{s}<1$, the range of maximum growth of this instability, one may usefully approximate

$k_{z}(z)=\left[\omega-l \omega_{R}(z) \pm \bar{\omega}(z) \pm \delta(z)\right] / v_{\alpha}^{0}$

with

$$
\begin{aligned}
\delta(z)= & \left.\frac{\epsilon \omega_{\rho}^{2} \omega_{\theta \mathrm{V}}^{2}(z) k_{\perp}^{2}\left(v_{g}^{0}\right)^{2}}{\bar{\omega}(z)}\left[\omega-l \omega_{R}(z) \pm \bar{\omega}\right)^{2}+\left(v_{\leftrightarrow}^{0}\right)^{2} k_{\perp}^{2}\right]^{-1} \\
& \times\left[\omega_{\rho}^{2}-\omega_{o v}^{2}(z)\right]^{-1}
\end{aligned}
$$

$\omega_{\mathrm{av}}=2 \omega_{R}-\boldsymbol{\Omega}$,

where $\bar{\omega}=\omega,\left(\omega_{0 v}\right)$ and $\epsilon=+(-)$ for the Langmuir (cyclotron) modes and $+(-)$ refers to the slow (fast) modes of propagation. Equation (7) may be substituted into (6) to yield an analytic estimate for the local growth rate. The resulting expression is still rather lengthy, and we do not give it here. Rather, we present in the accompanying figure a comparison of a completely numerical evaluation of the growth rate $\gamma(z)$ with an evaluation based upon the approximation (7) for a field profile of the form $\Omega(z)=\Omega_{0} /(1+G z)$ with $\Omega_{0} / \omega$, $=3$ and $G=0.01$ in appropriately normalized units of length. The agreement is seen to be satisfactory.

A numerical study of (6) reveals the following general qualitative features of the instability: (1) Both the slow and fast modes may be driven unstable, depending upon the frequency regime considered: (2) the frequency regime for which the largest growth rates occur is such that $\omega / \omega_{p} \approx 1$ from

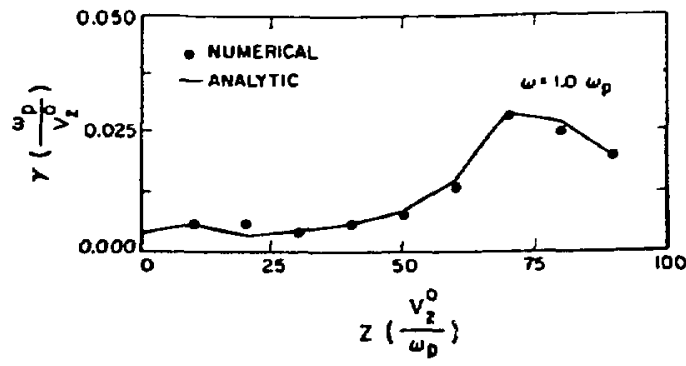

FIG. ?. Local growth rates for $k_{1}=0.1$ and $l=1$ mode.

which range the results presented in Fig. 1 are taken; (3) for this frequency it is the slow plasma mode which is most unstable; (4) the values of $k_{\perp}$ for which the maximum growth obtains are such that $k_{1} / k_{2}<1$; (5) these results may also be obtained from a purely analytic treatment based upon the estimate (7) for $k_{z}(z)$.

Our objective here has been to demonstrate that for a nonneutral electron beam propagating in an inhomogeneous magnetic field there is an instability whose existence may be revealed only through an analysis which explicitly includes the effects of such a gradient. No attempt has been made to evaluate the effects of this instability for particular electron beam devices. As applications of increasingly complex beam-field configurations are considered, it is perhaps well to remember that the stability of such configurations cannot always be completely evaluated by local versions of results derived for the case of a uniform magnetic field.

I wish to thank B. Godfrey and B. Newberger for numerous useful discussions throughout the course of this work. I am also grateful to D. DuBois, D. Forslund, J. Kindel, E. Lindman, and L. Thode for helpful comments.
${ }^{1}$ R. C. Davidson, Theory of Nomeutral Plasmas (Benjamin, Reading, Mass., 1974), and references therefn. ${ }^{2}$ M. I. Sloan and W. E. Drummond, Phys. Rev. Lett. 31, 1234 (1973).

${ }^{3}$ S. Yadavalli, Appl. Phys. Lett. 29, 272 (1976).
'T. Stix, Theory of Plasma Woves (McGraw-Hill, New York, 1962), p. 59.

${ }^{5}$ A. Bers and P. Penfield, Jr., IRE Trans. Electron. Devices $9,12(1962)$. 
[Submitted for publication.]

APPENDIX E

LINEAR THEORY OF RADIALLY INHOMOGENEOUS

UNNEUTRALIZED RELATIVISTIC ELECTRON BEAMS*

Brendan B. Godfrey

ABSTRACT

A set of four coupled first order differential equations describing the normal modes of a cylindrically symmetric, sold fluid, unneutralized relativistic electron beam of arbitrary radial profile is derived. Effects of beam rotation and equilibrium fields are treated exactly. The differential equations are found to have singular points for a radially inhomogeneous beam wherever the eigenfrequency equals the cyclotron or Doppler resonance frequencies. The resulting branch cuts in the dispersion function give rise to secularly decaying contributions to the initial value problem. The rate of decay and the character of the eigenmode near the singularity are determined from solution of the corresponding indicial equation. Discrete eigenmodes also exist, and are obtained by numerical solution of the differential equations and boundary conditions. For realistic solid beam equilibria, only one slow cyclotron mode exists for any given pair of axial and azimuthal wave numbers, and that mode is localized at the beam edge. Under identical conditions several slow spacecharge modes exist and are not so distorted. However, even at the spacecharge limit, the phase velocity of long wavelength $\mathrm{clow}$ spacecharge waves does not decrease to zero. These results are relevant to the Autoresonant and ConvergIng Guide collective ion acceleration proposals. 


\section{Introduction}

Collective ion acceleration is a highly speculative, yet potentially very significant, application of intense relativistic electron beams. Eventually, it may lead to compact and economical acceleration of substantial currents of light or heavy lons to hundreds of $\mathrm{MeV}$ per nucleon. Uses for such ion beams include controlled thermonuclear fusion, electronuclear breeding, basic nuciear physics, materials studies, and radiation therapy. Increased interest in collective ion acceleration is indicated by a growing number of experimental and theoretical sturies. 1

In the traveling wave class of collective acceleration mechanisms, slow Langmuir or cyclotron waves are exciced in the electron beam, ions are trapped In the electrostatic wells of these waves, and the waves plus ions are accelerated smoothly toward the beam velocity. The waves are grown in a reactive or resistive slow wave structure. Acceleration is achieved by spatial variation of external parameters affecting wave phase velocity, such as axial magnetic field strength ${ }^{2}$ or metallic drift tube radius. ${ }^{3}$ A thorough knowledge of the unneutralized relativistic beam linear eigenmodes is clearly important to each of these topics, as well as to the pure general issue of beam stability. Linear theory plays a vital role in the understanding of other intense relativistic electron beam applications, as well.

Previous theoretical investigations of unneutralized relativistic beam linear theory have, for the most part, ignored the intrinsic radial inhomogeneity of the beam equilibrium. ${ }^{4}$ As we shall see, radial inhomogeneity, partiaularly in particle kinetic energy, plays a decisive role in shaping the wave spectrum. In those instances in which the inhomogeneity was treated, other restrictive approximations were required in order to preserve analytical tr-atability. ${ }^{5-10}$ 
Here, we take the opposite approach, obtaining very general results, but at a cost. Although analytical insights are provided by our set of eigenmode equations, specific solutions are to be had only numerically. The advantage of this approach is that, once the radial eigenmode equations are implemented in a computer program, linear dispersion properties of electron beams in diverse parameter regimes can be determined with minimal additional effort. Precise Instability growth rates are particularly valuable in the planning of multidimensional computer simulations ${ }^{11-13}$ and $o .^{\cdot}$ experiments.

We begin in Sec. II by linearizing the relativistic cold fluid equations plus Maxwell's equations about an arbitrary cylindrically symetric beam equilibrium. (Cold fluid equations adequately describe most relativistic beam experiments, except when large scatter is deliberately introduced, as in certain microwa?e generation experiments. ${ }^{14}$ Finite Larmour radius modifications are discussed briefly in Sec. VI.) Arbitrary cylindrical boundary conditions exterior to the beam are permitted. Upon Fourier decomposing the Iinear equations in axial position, angle, and time, we are left with five first order differential equations in radius and five algebraic equations. A change of dependent variables, however, reduces these equations to a fourth order system. Examination of the coefficient of the fourth derivative shows it to vanish at radii corresponding to the Doppler and upper hybrid resonances.

These singular points of the coupled set of differential equations give rise to branch cuts in both the radial eigenmodes and the frequency spectrum. 15 The branch cuts contribute secularly decaying terms to the asymptotic solution of the Initial value probilem. ${ }^{16,17}$ Actual decay rates are determined by solution of the corresponding indicial equations. Physically, the branch cut contributions correspond to the stream lines in the beam each oscillating independently at a local resonance frequency, while the amplitudes gradually phase mix away. Details are in Sec. III. 
Sec. IV describes GPADR, a computer program for the solution of the eigenmode equations. From any given pair of constraints on the beam equilibrium, 18 beam and fleld radial profiles are computed iteratively. Based on these profiles, two independent solutions of the linear equations satisfying boundedness conditions on axis are integrated by a standard fourth order Runge-Kutta routine. $^{19}$ The determinant of the outer boundary conditions constitutes a dispersion function, the zeroes of which are the desired eigenfrequencies. A typlcal frequency-wavenumber diagram generated in this way is presented to show the dispersion of the various bean and waveguide modes and the branch cut intervals.

Two applications of this procedure to collective ion acceleration in solid beams are given in Sec. V. We find that, for any given pair of axial and azimuthal mode numbers, the upper hybrid branch cuts envelop all but the fundamental fast and slow cyclotron waves. Moreover, the slow cyclotron mode is strongly distorted into a surface wave. The Langmuir waves are not nearly so distorted or reduced in number by the Doppler branch cut. However, in contrast to the infinite guide field case, 20,21 the phase velocity of the Iong wavelength, fundamental slow Langmuir wave does not drop to zero even as the spacecharge limit is approached. Ramifications are discussed briefly. Ref. 11, 13 and 22 contain other applications of GRADR. 


\section{The Radiai Eigenmode Equations}

We begin from Maxwell's equations and the relatjvistic cold fluid equations, written in dimensionless form:

$$
\begin{aligned}
& \frac{\partial}{\partial t} B_{z}+\frac{1}{r} \frac{\partial}{\partial r} r E_{\Theta}-\frac{1}{r} \frac{\partial}{\partial \theta} E_{r}=0 \\
& \frac{\partial}{\partial t} B_{r}+\frac{1}{r} \frac{\partial}{\partial \Theta} E_{z}-\frac{\partial}{\partial z} E_{\Theta}=0 \\
& \frac{\partial}{\partial t} B_{\Theta}+\frac{\partial}{\partial z} E_{r}-\frac{\partial}{\partial r} E_{z}=0 \\
& \frac{\partial}{\partial t} E_{z}+\frac{1}{r} \frac{\partial}{\partial \theta} B_{r}-\frac{1}{r} \frac{\partial}{\partial r} r B_{\theta}+n u_{z}=0 \\
& \frac{\partial}{\partial t} E_{r}+\frac{\partial}{\partial z} B_{\Theta}-\frac{1}{r} \frac{\partial}{\partial \theta} B_{z}+n u_{r}=0 \\
& \frac{\partial}{\partial t} \mathbf{E}_{\Theta}+\frac{\partial}{\partial r} B_{z}-\frac{\partial}{\partial z} B_{r}+n u_{\theta}=0 \\
& \gamma \frac{\partial}{\partial t} u_{z}+u_{z} \frac{\partial}{\partial z} u_{z}+u_{r} \frac{\partial}{\partial r} u_{z}+u_{\Theta} \frac{1}{r} \frac{\partial}{\partial \theta} u_{z}=\gamma E_{z}+u_{r} B_{\theta}-u_{\Theta} B_{r} \\
& \gamma \frac{\partial}{\partial t} u_{r}+u_{z} \frac{\partial}{\partial t} u_{r}+u_{r} \frac{\partial}{\partial r} u_{r}+u_{\Theta} \frac{1}{r} \frac{\partial}{\partial \theta} u_{r}=\gamma E_{r}+u_{\Theta} B_{z}-u_{z} B_{\Theta}+\frac{1}{r} u_{\theta}^{2} \\
& \gamma \frac{\partial}{\partial t} u_{\Theta}+u_{z} \frac{\partial}{\partial z} u_{\Theta}+u_{r} \frac{\partial}{\partial r} u_{\Theta}+u_{\Theta} \frac{1}{r} \frac{\partial}{\partial \theta} u_{\Theta}=\gamma E_{\Theta}+u_{z} B_{r}-u_{r} B_{z} \\
& \frac{\partial}{\partial t} \gamma_{n}+\frac{\partial}{\partial z} u_{z} n+\frac{1}{r} \frac{\partial}{\partial r} r u_{r} n+\frac{1}{r} \frac{\partial}{\partial \theta} u_{\theta} n=0
\end{aligned}
$$

The relativistic particle energy $\gamma$ is defined as

$$
r^{2}=u_{z}^{2}+u_{r}^{2}+u_{\theta}^{2}+1
$$


In these equations $\mathrm{n}$ is the beam density as determined in a local rest frame and is related to the measurable density by $\mathrm{n}=\mathrm{n}_{\ell a b} / \gamma$. Similarly, $\mathrm{u}$ is the relativistic momentum, $u=\gamma v$. We have found that Eq. (1)-(3) lead to simpler linearized equations than do a number of alternate representations.

In cylindrical equilibrium, only $n^{\circ}, u_{z}{ }^{\circ}, u_{\Theta}{ }^{\circ}, B_{z}{ }^{\circ}, B_{\Theta}{ }^{\circ}$, and $E_{r}{ }^{\circ}$ are non-zero. They are related by Eq. (1) and (2).

$$
\begin{aligned}
& \frac{1}{r} \frac{d}{d r} r B_{\Theta}^{\circ}-n^{\circ} u_{z}^{0}=0 \\
& \frac{d}{d r} B_{z}^{\circ}-n^{\circ} u_{\Theta}{ }^{\circ}=0 \\
& \gamma E_{r}^{\circ}+u_{\Theta}{ }^{\circ} B_{z}^{\circ}-u_{z}{ }^{\circ} B_{\Theta}^{\circ}+\frac{1}{r} u_{\Theta}{ }^{2}=0 \\
& \frac{1}{r} \frac{d}{d r} r E_{r}^{\circ}-\gamma^{\circ} n^{\circ}=0
\end{aligned}
$$

Two of the six quantities can be specified arbitrarily. 18

Linearization of Eq. (1)-(3) followed by Fourier transformstion in z, $\theta$, and $t$ gives five first order differential equations in radius and five algebraic equations. Their structure is clearest in matrix format, $A \cdot x=0$. Table 1 lists the coefficients $A_{i j}$ and the dependent variables $X_{i}$ and defines several commonly occurring algebraic terms. Typically, $\eta_{z} \sim 0$. Frequency, axial wavenumber, and azimuthal mode number are $\mathrm{w}, \mathrm{k}$, and $\mathrm{m}$.

$$
\Omega=\omega \gamma^{0}-k u_{z}^{0}-\frac{m}{r} u_{\Theta}^{0}
$$

is the Doppler-shificed frequency.

Although the system of equations appears to be fifth order, the minor determinant conjugate to the five derivatives in Table 1 vanishes identically. 
The system is, therefore, no greater than fourth order, and, as it stands, cannot be integrated numerically. To reduce by one the number of derivative terms, we first eliminate $(i \cdot n)$ from the equations and then perform the variable chenges

$$
\begin{aligned}
& \left(i \cdot \bar{B}_{\theta}\right)=\left(i \cdot B_{\theta}\right)+\left(n^{0} u_{r}\right) u_{z}{ }^{\circ} / \Omega \\
& \left(i \cdot \bar{B}_{z}\right)=\left(i \cdot B_{z}\right)-\left(n^{0} u_{r}\right) u_{\theta} 0 / \Omega .
\end{aligned}
$$

Note that $u_{r} / \Omega$ is the radial displacement of stream lines, so the transformation (6) has the effect of subtracting from $B_{\Theta}$ and $B_{z}$ the perturbed surface current contribution. Hence, $\bar{B}_{\theta}$ and $\bar{B}_{z}$ are continuous across the beam edge. An analogous transformation on $E_{r}$ exists but is of no purpose here, since the system of equations does not involve derivatives of $E_{x}$.

From these manipulations and a minor rearrangement of terms, we obtain Table 2, with

$$
\Delta=\Omega^{2}-n^{0}-\xi_{z} n_{z}-\xi_{\theta} n_{\theta}
$$

This quantity vanishes, to lowest order, when

$$
\Omega \approx \pm\left[\left(\mathrm{B}_{z}{ }^{\circ}\right)^{2}-\mathrm{n}^{\circ}\right]^{1 / 2}
$$

defining the cyclotron, or upper hybrid, resonances. The difference in sign of $\mathrm{n}^{\circ}$ in Eq. (8), relative to the homogeneous plasma case, is due to beam rotation. It persists in beam nonlinear envelope equations. 12 The minor determinate conjugate to the four derivatives in Table 2 is equal to $\omega^{2} \Omega^{2} \Delta$, which is not identically zero unless $\omega=0$. The efgenmode equations constitute a fourth order system. Two boundary conditions must be imposed at $r=0$ and at $r=R$, some exterior surface. 
It can be shown formally that the factor ()$^{2}$ in the minor determinant also multiplies all other coefficients of the fourth order differential equation, and, hence, does not affect the order of the system. The factor simply cancels, even in the limit of zero frequency. 
III. Branch Cuts and Indicial Equations

For given $k$ and $m$, there are at any radius $x$ within the beam precisely one value of $\omega$ for which $\Omega$ vanishes and two values for which $\Delta$ vanishes. (Outside the beam, $n^{0}=0$, the quantities $\Omega$ and $\Delta$ are oi no consequence, because they multiply perturbed momenta, which are identically zero there.) The vanishing of $\Omega$ or $\Delta$ at some $r$ defines it as a singular point of the differential equations: At least one solution to the equations has a branch point there. 23,24 Because singularities play a singificant and, perhaps, startling role in the linear theory, we examine them in some detail.

The behavior of solutions to the differential equations near singularities are determined by the roots of the corresponding indicial determinants. 24 The calculations are straightforward, though tedious. It is more instructive First to obtain simplified, approximate sets of differential equations valid near the singular points. For $\Omega \approx 0$, we find

$$
\begin{aligned}
& {\left[\Delta \frac{d}{d r}-n^{0} \frac{\Sigma}{\Omega}\right] i \bar{B}_{-}+\left[n^{0}\left(\omega^{2}-k^{2}-\frac{m^{2}}{r^{2}}\right) \frac{\Delta}{\Omega^{2}}-n^{0}\left(k \eta_{z}+\frac{m}{r} \eta_{\Theta}\right) \frac{\Sigma}{\Omega^{2}}\right] E_{+}=0,} \\
& {\left[\Delta+n^{\circ}\right] i \bar{B}_{-}+\left[\Delta \frac{d}{d r}+n^{\circ}\left(k \eta_{z}+\frac{m}{r} \eta_{\Theta}\right) \frac{1}{\Omega}\right] E_{+}=0,}
\end{aligned}
$$

where

$$
\begin{aligned}
& \mathrm{E}_{ \pm}=\mathrm{E}_{z} / \zeta_{z} \pm \mathrm{E}_{\theta} / \zeta_{\theta}, \\
& \overline{\mathrm{B}}_{ \pm}=\overline{\mathrm{B}}_{z} / \mathrm{u}_{\theta}{ }^{0} \pm \overline{\mathrm{B}}_{\Theta} / \mathrm{u}_{\mathrm{z}}{ }^{0},
\end{aligned}
$$

and

$$
\Sigma=\frac{d}{d r} \Omega-\xi_{z} n_{z}-\xi_{\theta} n_{\theta}
$$


Thus, two of the four independent solutions of the differential equations are regular at $\Omega\left(\mathrm{r}_{\mathrm{o}}\right)=0$, while two go as

$$
E_{+} \sim\left(r-r_{0}\right)^{s}
$$

with s a solution of

$$
\begin{aligned}
& \Delta\left(s \frac{\mathrm{d}}{\mathrm{dr}} \Omega\right)^{2}+\left(s \frac{\mathrm{d}}{\mathrm{dr}} \Omega\right)\left[\mathrm{n}^{\circ}\left(\mathrm{k} \eta_{\mathrm{z}}+\frac{\mathrm{m}}{\mathrm{r}} \eta_{\Theta}\right)-\mathrm{n}^{\circ} \Sigma-\Delta \frac{\mathrm{d}}{\mathrm{dr}} \Omega\right] \\
& -\mathrm{n}^{\circ}\left[\left(\frac{\mathrm{d}}{\mathrm{dr}} \Omega-\Sigma\right)\left(\mathrm{k} \eta_{\mathrm{z}}+\frac{\mathrm{m}}{\mathrm{r}} \eta_{\Theta}\right)+\left(\mathrm{n}^{\circ}+\Delta\right)\left(\omega^{2}-\mathrm{k}^{2}-\frac{\mathrm{m}^{2}}{\mathrm{r}^{2}}\right)\right]=0 .
\end{aligned}
$$

The quantities $\overline{\mathrm{B}}_{-}, \mathrm{E}_{\mathrm{r}}, \mathrm{u}_{z}, \mathrm{u}_{\theta}$, and $\mathrm{n}$ vary as the $\mathrm{s}-1$ power of $\mathrm{r}-\mathrm{r}_{0}$; $\overline{\mathrm{B}}_{+}$, $B_{r}$, and $u_{r}$, along with $E_{+}$in $(12)$, vary as the $s$ power; and $E_{-}$varies as the $s+1$ power; as may be determined from Eq. (o; and Table 2 .

In contrast, we find for $\Delta \approx 0$ a single first order equation.

$$
\begin{aligned}
& \frac{d}{d r}\left(n^{0} u_{r}^{0} \Delta\right)=\frac{n^{0}}{\Delta}\left[\left(u_{z}^{0}-\frac{k}{\Omega}\right) \eta_{z}+\left(u_{\theta}^{0}-\frac{m}{r \Omega}\right) \eta_{\theta}+\right. \\
& \left.\frac{r^{0} \Omega}{\omega}\left(\zeta_{z} \frac{d}{d r} \frac{u_{z}^{0}}{\Omega}+\zeta_{\Theta} r \frac{d}{d r} \frac{u_{\Theta}^{0}}{r \Omega}\right)-\zeta_{z} \xi_{z}-\zeta_{\Theta} \xi_{\theta}\right]\left(n^{0} u_{r} \Delta\right)
\end{aligned}
$$

One of the four independent solutions of Table 2 varies as

$$
\mathbf{n}^{\circ} \mathbf{u}_{\mathbf{r}} \Delta \sim\left(\mathbf{r}-\mathbf{r}_{\mathbf{0}}\right)^{s}
$$

where $s$ is now the coefficient of (n $r \Delta$ ) in Eq. (14) divided by the logarithmic derivative of $\Delta$. Therefore, $n$ varies as the $s-2$ power of $r-r_{0} ; E_{r}$, $u_{z}, u_{\theta}$, and $u_{r}$ vary as the $s-1$ power; and $E_{z}, E_{\theta}, \vec{B}_{z}, \vec{B}_{\theta}$, and $B_{r}$ vary as the s power. The other three independent solutions are regular at $\Delta\left(r_{0}\right)=0$. 
Under fairly general conditions, Eq. (13) can be simplified to give

$$
\mathrm{s} \approx \frac{1}{2} \pm\left[\mathrm{n}^{\circ}\left(\mathrm{k}^{2}-\omega^{2}\right)\right] / \frac{\mathrm{d}}{\mathrm{dr}} \Omega
$$

if $m=0$. Since $\Omega \sim \mathbf{r}^{2}$ for realistic solid beam equilibria, $s$ is predominantlv imaginary and large for small $r$. We have been unable to find a simplified form for $s$ at the cyclotron resonance. However, it is purely real and, for the equilibria treated in Sec. IV, very small. Fig. 1 plots frequencies zequired for $\Delta\left(r_{0}\right)=0$ and $\Omega\left(r_{0}\right)=0$. Also plotted are $s$ for the cyclotron resonances and $|\mathrm{Im}[\mathrm{s}]|$ for the spice charge resonance. For the latter resonance, $\operatorname{Re}[\mathrm{s}] \doteq 0.5$. Our results for the cyclotron resonance are in good agreement with the analytic estimate of Ref. 10, which gives $s=0$ in the large $\gamma$ 1imit.

Barston has shown that singular solutions to sets of linear differential equations generally can be made to satisfy the required boundary conditions at each edge of the region of integration, because not all the field components need be continuous across the singular point. ${ }^{15}$ Thus, the beam exhibits three continua of singular eigenmodes with corresponding bands of eigenfrequencies, as in Fig. 1. The loci of continuum eigenfrequencies constitute branch cuts in the dispersion function $D(w, k, m)$. The perturbed momenta of these eigenfunctions are sharply peaked about their singular radil. The modes may be thought of as Individual stream lines each oscillating at the local value of the Doppler or cyclotron resonance frequency.

This physical picture is brought into sharper focus by the work of Sedlacek, who performed a Fourier time inversion on a continuum of eigenmodes having logarithmic singulartities. ${ }^{16}$ The resulting solution to the initial value problem contains terms of the form 


$$
\psi(x) \sim t^{-1} e^{i \omega(x) t}
$$

where $\omega(x)$ is the local resonance frequency. The secular decay with time results from phase mixing of adjacent stream lines. Repeating the calculation for the present problem gives this same behavior, but with envelopes decaying as $t^{-s-1} \cdot 25$ Excitation and subsequent secular decay of stream line oscillations in analogous spatial initial value problems have been observed in computer simulations of electron beams. ${ }^{12}$ Wave energy appeared to dissipate into electron thermal motion.

Because of their large bandwidth, continuum modes rather than discrete modes tend to be exciter by external perturbations of relativistic beam equilibria. As we shall see in Sec. IV and V, this effect Is enhanced by the fact that many eigenmodes present in homogeneous plasmas are supplanted in solid beam equilibria by the continuum modes. Those discrete modes which would otherwise lie in the continuum frequency bands instead pass through the branch cuts onto other sheets of the dispersion function, where they are heavily damped and of 1ittle consequence. 16 
IV. Numerical Methods and Solutions

A computer program, GRADR, has been written to solve numerically the equilibrium, linear, and indicial equations presented in the two preceeding sections. Although two of the six equilibrium quantities in Eq. (4), e.g., the energy and dersity radial profiles, are freely specifiable, we wish to choose values physically realizable and approriate to collective ion acceleration. The first constraint is obvious: Total particle energy, kinetic plus potential, must be constant across the beam. As a second constraint, we let the current density be constant. Though a bit arbitrary, the choice is realistic for solid beams in a strong magnetic guide field. (For a weak guide field bean expansion after leaving the diode can be treated approximately by conservation of canonical angular momentum. ${ }^{22}$ )

The system of Eq. (3), Eq. (4), and the two constraint equations is solved iteratively, starting from a rigid rotor equilibrium. 4 Convergence is rapid except for currents very near the spacecharge limit. In that case a few thousand iteracions and a small relaxation parameter are needed to achieve accuracy of one part in $10^{5}$. Of course, for currents above the spacecharge 1imit no equilibrium exists, and the iterative pracess diverges. Incidentally, such computations can provide an accurate estimate of the spacecharge limiting current for a beam of specified current profile, ${ }^{22}$ without the usual assumptions on the magnitude of the drift tube to beam radii ratio, $R / a,{ }^{26}$ or of the guide field strength, $B_{z} \circ 27,28$

Fig. 2 shows calculated equilibrium profiles for the $3 \mathrm{MeV}, 30 \mathrm{kA}$ electron beam to be used by Austin Research Associates, Inc. In an upcoming autoresonant acceleration experinent. 29 The drift tube radius 
shown here, twice the beam radius, is about $40 \%$ larger than that called for by present experimental plans. 11 Dashed lines in three of the plots represent particle quantities extrapolated into the vacuum region. Test particles in equilibrium outside the beam would have those parameters. The beam density, as measured in the lab frame, (not shown) is approximately unity. The branch cut results of Fig. 1 are based on this equilibrium.

With an equilibrium given numerically, GRADR obtains linear eigenmodes and eigenfrequencies as follows. The system of equations represented by Table II is integrated from the axis outward by a standard fourth order Runge-Kutta routine, ${ }^{19}$ because this approach is convenient and economical. For an integration of the eigenmode equations, four constants must be specified at a point. Boundedness at the axis,

$$
\frac{d}{d z} E_{z}, \frac{d}{d z} \bar{B}_{z} \sim r^{|m|+1},
$$

prescribes two of the constants. The other two must be selected, if posstble, so that boundary conditions at $r=R$ are satisfied. At a metal drift tube,

$$
\mathrm{E}_{\mathbf{z}}, \mathrm{E}_{\theta}=\mathbf{0}
$$

So, choose a triple $(6, k, m)$ and integrate two independent solutions of the differential equations based on two arbitrary but independent sets of constarts on axis. If $(\omega, k, m)$ correspond to an elgenmode, then a 
linear combination of the two solutions must satisfy (19). Typically, this does not occur, and the determinant of the boundary conditions at $r=R$ does not vanisk. Therefore, the problem at hand is to vary $\omega$ unti] the determinant, which is closely related to the dispersion function $D(\omega, k, m),{ }^{17}$ is sufficiently small. Use of Muller's method ${ }^{29}$ often achieves convergence of $\omega$ to a relative accuracy of $10^{-5}$ with but five evaluations of the determinant.

Incidentally, an equivalent procedure involves integrating from $r=0$ two solutions satisfying (18) and from $r=R$ two solutions satisfying (19). Vanishing of the Wronskian of the four solutions indicates that an eigenmode has been found. ${ }^{16}$ Although conceptually more satisfying, this second approach is computationally less convenient. We should romark that the numerical procedure, as described, is suitable only for discrete roots on the first Riemann sheet of the dispersion function. Finding discrete roots on other sheets, which are of little interest, would require contour integrations in the complex $r$ plane. It is not clear whether the so-called continuum spectra can be obtained by this means.

Fig. 3 is an $\omega-k$ diagram for $m=0$ computed for the equilibrium of Fig. 2. The three shaded bands are the regions of continuum eigenmodes, while lines indicate discrete normal modes. The two curves enter'.ng at the top (and bottom) are the lowest TE and TM cavity modes. The TM mode, after disappearing into the cyclotron resonance band, reappears on the far side. Irmediately below it is the node-free fast cyclotron mode. (Note, though, that the properties of the fundamental TM and cyclotron modes are interchanged in this region.) Additional 
fast cyclotron waves with progressively mode radial nodes, as occur in homogeneous plasmas, do not exist in solid beams with realistic $\gamma$ profiles due to the broad resonance region. Very near the spacecharge resonance band are the fast and slow Langmuir waves with no radial nodes. A few other langmuir waves, not shown, lie still closer to the resonance region. Their number is sma11, of order five on each side. The remaining mode, just above the lower cyclotron resonance and arbitrariiy close to it as $k \rightarrow \infty$, is the sole slow cyclotron wave. Since the s1ow Langmuir and cyclotron modes are central to traveling wave collective ion arceleration proposals, we discuss them further in the next section. 


\section{v. Appifcations to Collective Ion Acceleration}

The autoresonant accelerator proposal employs axi-symmetric slow cyclotron waves in an unneutralized intense relativistic electron beam. ${ }^{2}$ In a homogeneous plasma, the dispersion relation of this mode is approximately $y^{12}$

$$
\omega \gamma^{0}=k u_{z}^{0}-B_{z}^{0} \frac{k^{2}+k_{1}^{2}}{k^{2}+k_{1}^{2}+n^{0}},
$$

with $k_{1}$ the perpendicular (here, radial) wave number. In a beam the cyclotron wave basically is a traveling pinch, and a deep electrostatic well occurs where the beam is most constricted. Acceleration of waves and lons trapped in them is accomplished by having the waves propagate along the beam into regions of progressively weaker $\mathrm{B}_{\mathrm{z}}{ }^{\circ}$, causing $\mathrm{k}$ to decrease and the phase velocity to increase.

In radially inhomogeneous beams no:e of the equilibrium quantities in Eq. (20) is constant, and we should expect the dispersion properties of the slow cyclotron wave to be modified somewhat. Indeed, one change has already been noted in Sec. IV. In a homogeneous beam $k_{1}$ can assume a countably infinite set of values, corresponding to the number of radial nodes in $E_{z}$. Here, only one slow cyclotron mode exists, the rest presumably enveloped by the cyclotron branch cut. The closeness of the solitary cyclotron mode to the $r=a$ branch point in Fig. 3 suggests ar Improved approximate dispersion relation, simply

$$
\Delta(a)=0
$$

In other words, according to Eqs. (21) and (3), the wave frequency lies 


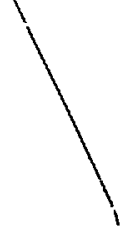

very near the upper hybred resonance, defined using the value of $\gamma^{\circ}$ at the beam edge.

The proximity of the cyclotron wave and the branch cut also gives rise to significant distortion of the eigenmode profile. See Fig. 4. In contrast, the profiles are Bessel functions ${ }^{30}$ in a homogeneous beam. The strong enhancement of the perturbed fluid quantities, especially $u_{r}$, at the beam surface is worrisome in that it may lead to stream line crossing and turbulence at comparatively low $\mathrm{E}_{z}$. We hope to perform two-dimensional computer simulations ${ }^{31}$ in the near future in order to investigate the nonlinear aspects of this behavior.

Examination of Eq. (7) indicates that for solid beams variation in $\Delta$ typically is due primarily to variation in $\gamma^{\circ}$. Because the change in $\gamma^{\circ}$ across the beam is of order $\nu$, Budker's dimensionless current parameter, ${ }^{32}$ one might hope to minimize the adverse inhomogeniety effects upon cyclotron waves by reducing $\nu / \gamma^{\circ}$. It does not work. In a homogeneous beam $k_{1}^{2}$ ranges from roughly $v^{-1}$ to infinity, giving, according to Eq. (20), a band of cyclotron waves of width $\cup \mathrm{B}_{\mathrm{z}}{ }^{\mathrm{o}} /\left(\gamma^{0}\right)^{2}$. Up to factors of order unity, this is also the width of the cyclotron branch cut. Therefore, the two scale together, and the branch cut always replaces all but the fundamental cyclotron mode in a real beam of relativistic energy. This crude argument has been verified by GRADR calculations with $\nu / Y^{\circ}$ as sma11 as 0.02 .

That the remaining mode is localized at the beam edge suggests that a gradual reduction of $\mathrm{n}^{\circ}$ to zero there, rather than the sharp discontinuity in Fig. 2, might cause that mode to vanish too. ${ }^{15}$ Using a trapezoidal profile, we have, in fact, found the fundamental mode to 
pass through the branch cut onto a second Riemann sheet when the density scale length exceeds about $1 / 3$ the beam radius. Keeping the gradient scale length small should not prove too difficult in practice. Incidentally, the effects described above for $\mathrm{m}=0$ slow cyclotron waves are enhanced with increasing $|\mathrm{m}|$.

The converging guide accelerator proposal, on the other hand, employs slow Langmuir waves. ${ }^{3,33}$ For ka small the phase velocity of the fundamental slow Langmuir mode is a strong function of R/a through $\gamma^{\circ}$. Axial variation of $R$ provides acceleration. The waves are predominately compressiunal. Insofar as inhomogeniety effects are concerned, slow Langmuir waves differ from slow cyclotron waves in two significant respects: The Doppler resonance band is relatively much narrower than the upper hybred resonance bands, and the slow Langmuir modes are located in Fig. 3 near the $r=0$ rather than the $r=a$ end of the resonance region. Consequently, several modes survive, and the slow waves are not strongly distorted. Details of the equilibrium density profile do not appear to affect these conclusions.

The linear eigenmode is plotted in Fig. 5 for $\mathrm{ka}=0.265$. Note that the axial accelerating field $E_{z}$ is comparatively sma11. Larger ka enhances the electric field but also raises the phase velocity. $\mathrm{ka} \approx 1$ is a reasonable compromise.

Analytic calculations demonstrate that, in the infinite guide field limit, the wave phase velocity drops to zero only as the spacecharge 1imit is reached. 20,21 It may be, therefore, that the very low phase velocities necessary to trap stationary ions cannot in practice be achieved. An ion injector would be required. GRADR computations show that with finite $\mathrm{B}_{z}{ }^{\circ}$ the situation is a bit worse. Fig. 6 gives the 
phase velocities of the fundamental fast and slow Langmuir waves as a function of $\mathrm{R} / \mathrm{a}$ for $\mathrm{B}_{\mathrm{z}}{ }^{\circ}=2$. (For a $2.65 \mathrm{~cm}$ radius beam, this corresponds to $3.4 \mathrm{~kg}$ ) ) Determination of minimum phase velocity for varying guide field strength would be: an interesting and useful exercise. 


\section{vI. Concluding Comments}

In this report we have described in broad terms the properties of linear eigenmodes of a cold fluid, solid, unneutralized, Intense, relativistic electron beam. The wave frequency spectrum is dominated by broad Doppler and upper hybred resonances. Due to their influence only a few beam modes with low radial node numbers exist, and some of these are transformed into surface waves. Distortion is particularly severe for the cyclotron wave to be used in autoresonant acceleration and conceivably can cause difficulties. Low Langmuir wave phase velocity desirable in the initial stage of converging guide acceleration is achievable only with a strong magnetic guide field, if at all.

If, in fact, the influence of energy inhomogeniety proves burdensome for nonlinear cyclotron waves, it may br. necessary to employ partially hollow beams. It is possible to live with a large minimum phase velocity of Langmuir waves, if required.

Throughout this investigation we have ignored the influence of beam scatter, non-zero particle Larmour radius. Mathematically, an effective transverse temperature converts our fourth order system of differential equations to a sixth order set. The coefficients of the additional two derivitives are proportional to the Larmour radius, $r_{L}$. Branch cuts, therefore, are replaced by a large number of discrete modes. ${ }^{34}$ For $r_{L} \ll a$, as is almost always true, the new modes should prove to be highly localized, with an effective radial width of order the Larmour radius. Frequency spacing would also be small. Thus our physical picture remains intact: Excitation of the resonance band causes each stream line to oscillate at its local characteristic frequency, while the envelope decreases due to phase mixing. (Recurrence of the initial 
state after a very long time is precluded by Landau damping, 34 Note, though, that scatter occasionally can drive instabilities. ${ }^{14}$ ) The fundamental slow cyclotron mode might be broadened a bit in radius, but not significantly in realistic circumstances. 


\section{Acknowledgments}

It is a pleasure to acknowledge the collaboration of W. A. Proctor and T. C. Genoni during an early portion of this research. Numerical methods were developed with the assistance of B. S. Newberger. These three together with R. J. Faehl, R. B. Miller, W. R. Shanahan, and L. E. Thode provided advice and encouragement throughout the course of the work. 


\section{Table Captions}

Table 1. Matrix representation of radial eigenmode equations in original form. Differential operators in $A_{i j}$ end in a centered dot. Nu11 elements are omitted.

Table 2. Matrix representation of radial eigenmode equations in a form suitable for numerical solution. The tenth equation, which determines ( $i \mathrm{n}$ ), has been decoupled. See Table 1 for definitions of special symbols. 


\section{Ref rences}

* Research supported jointly by the Air Force Office of Scientific Research and the Energy Research and Development Administration.

1 See, e.g., papers from the 1977 Particle Accelerator Conference (Chicago, 16-18 March 1977), IEEE Nuc. Sci. 24, 1625-1667 (1977).

2. M. L. Sloan and W. E. Drumnond, Phys. Rev. Lett. 31, 1234 (1973).

3. P. Sprangle, A. T. Drobot, and W. M. Manheimer, Phys. Rev. Lett. 36,1880 (1976).

4. See R. C. Davidson, Theory of Nonneutral Plasmas (Benjamin, Reading, 1974) and references therein.

5. R. H. Levy, Phys. Fluids 11, 920 (1968).

6. R. J. Briggs and S. F. Paik, Int. J. Electronics 23, 163 (1968).

7. R. J. Briggs, J. P. Daugherty, and R. H. Levy, Phys. Fluids 13, 421 (1970).

8. J. A. Rome and R. J. Briggs, Phys. Fluids 15, 796 (1972).

9. T. M. Antonsen and E. Ott, Phys. Fluids 18, 1197 (1975).

10. W. E. Drutamond, G. I. Bourianoff, E. P. Cornet, D. E. Hasti, W. W. Renstra, M. L. Sloan, H. V. Wong, J. R. Thompson, and J. R. UgJ.am, "A Theoretical Investigation of the Principal of Auto-Resonant Acceleration," AFWL-TR-75296 (Air Force Weapons Laboratory, Albuquerque, 1976), App. B.

11. R. J. Faeh1, B. B. Godfrey, B. S. Newberger, W. R. Shanahan, and L. E. Thode, IEEE Nuc. Sci. 24,1637 (1977).

12. B. B. Godfrey, IEEE Plas. Sci., to be published.

13. B. B. Godfrey, R. J. Faehl, B. S. Newberger, W. R. Shanahan, and L. E. Thode, Proc. Second Int. Conf. Electron and Ion Beam Research and Technology (Cornel1 University, Ithaca, 1977), to be published.

14. P. Sprangle and A. T. Drobot, IEEE Microwave Theo. Tech. 25, 528 (1977).

15. E. M. Barston, Ann. Phys. 29, 282 (1.964).

16. Z. Sedlacek, J. Plas. Phys. 5,239 (1971); $\underline{6}, 187$ (1971).

17. Z. Sedlacek, Czech. J. Phys. 22B, 67 (1972); 22B, 439 (1972); 23B, 892 (1973).

18. Ref. 4, Sec. 2.1, pp. i7-21.

19. M. Abramowitz and I. A. Stegun, Handbook of Mathematical Functions, AMS 55 (U. S. Government Printing Office, Washington, 1965), Sec. 25.5, p. 896. 
20. R. J. Briggs, Phys, Fluids, 19, 1257 (1976).

21. B. B. Godfrey, IEEE Plas. Sci., to be published.

22. I. E. Thode, B. B. Godfrey, and W. R. Shanahan, in preparation.

23. P. M. Morse and H. Feshbach, Methods of Theoretical Physics (McGraw-Hill, New York, 1953), Vol. 1, Ch. 5.2.

24. E. L. Ince, Ordinary Differential Equations (Dover, New York, 1956), Ch. 15.

25. M. J. Lighthill, Fourier Analysis and Generalized Functions (Cambridge University Press, London, 1964), p. 43 .

26. L. S. Bogdankerich and A. A. Rukhadze, Sov. Phys. Uspekhi 14, 163 (1971).

27. J. R. Thompson and M. L. Sloan, Proc. Second Int. Conf. Electron Ion Beam Research and Technology (Cornell Uriversity, Ithaca, NY, 1977), to be published.

28. W. A. Proctor and T. C. Genoni, in preparation.

29. J. F. Traub, Iterative Methods for the Solution of Equations (PrenticeHa11, Englewood Cliffs, 1964), Ch. J.0.

30. Ref. 19, Ch. 9 .

31. B. B. Godfrey and W. R. Shanahan, in N. G. Cooper (ed.), "Theoretical Division Annual Repo t, July 1975-September 1976," LA-6816-PR (Los Alamos Scientific Laboratory, 1977), p. 147.

32. G. I. Budker, Atom. Energ. 1, 9 (1956).

33. R. Adler, G. Gammel, J. A. Nation, M. E. Read, R. Williams, P. Sprangle, and A. Drobot, Proc. Second Int. Conf. Electron and Ion Beam Research and Technology (Cornoll University, Ithaca, NY, 1977), to be published.

34. J. H. Brownell, J. Plas. Phys. 9, 275 (1973); Phys. Fluids 16, 1167 (1973i。 


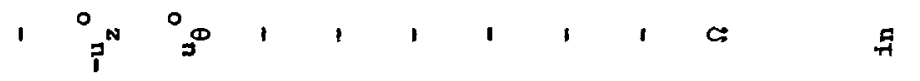

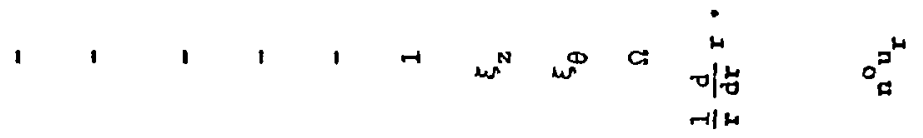

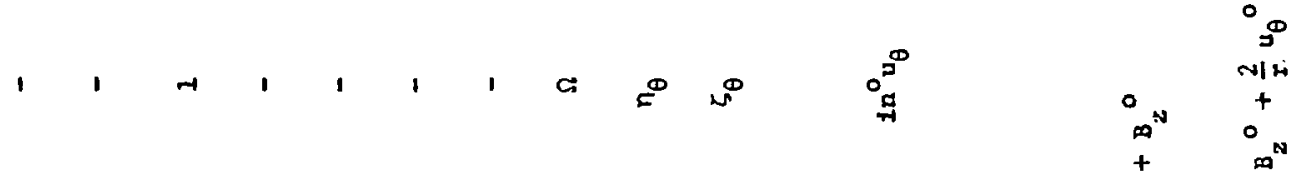

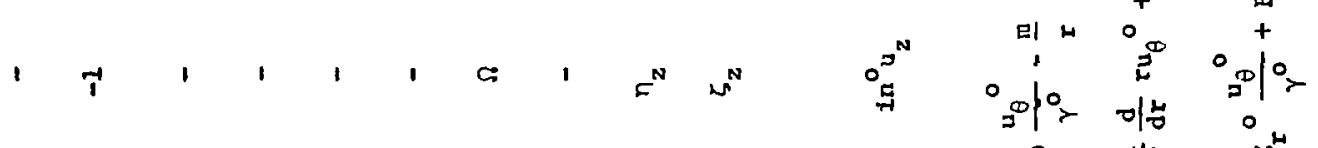

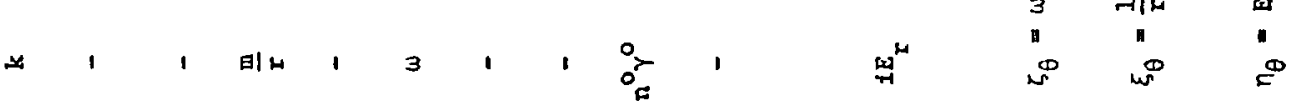

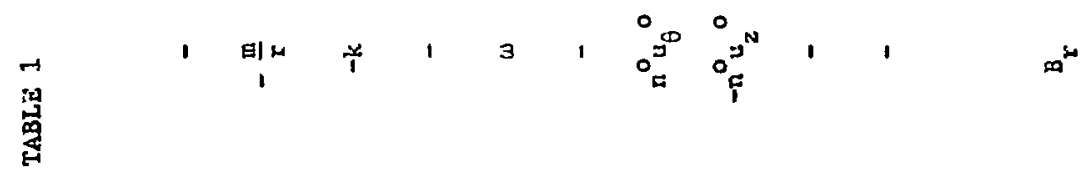

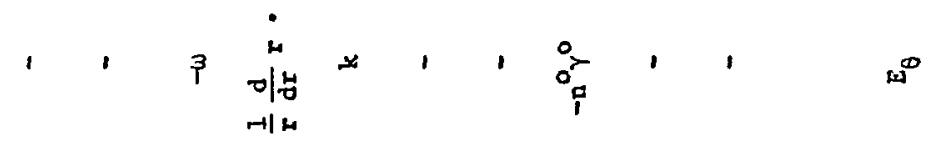

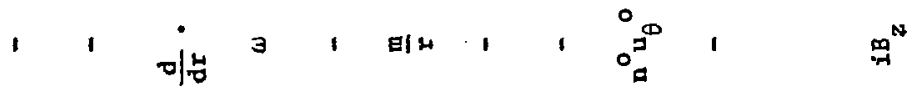

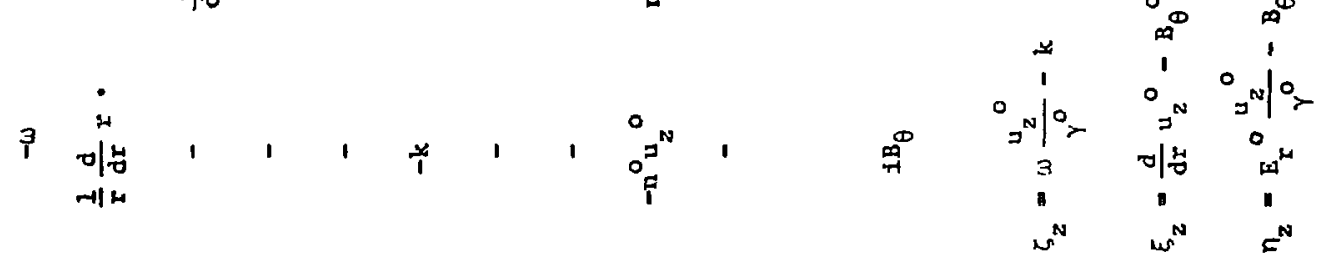

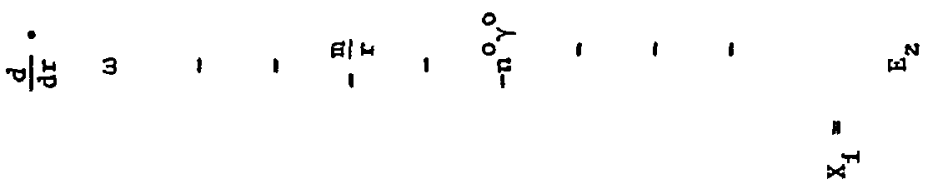




$$
\begin{aligned}
& \begin{array}{llllllllll}
1 & 1 & 1 & 1 & 1 & 1 & 1 & 1 & 1 & 0
\end{array}
\end{aligned}
$$

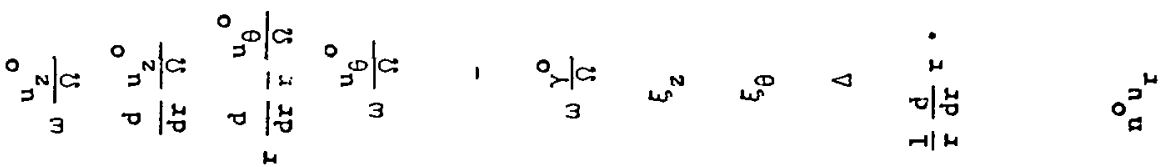

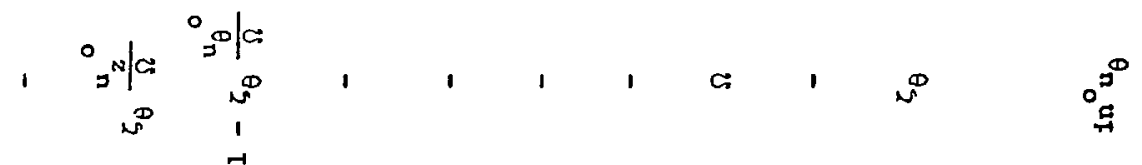

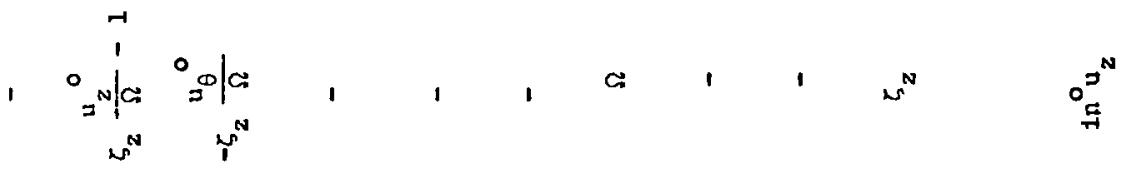

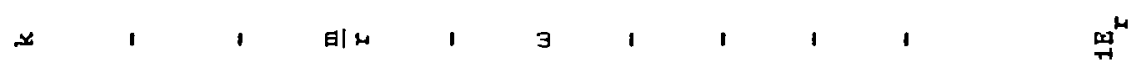

$$
\begin{aligned}
& \text { 要 } 1
\end{aligned}
$$

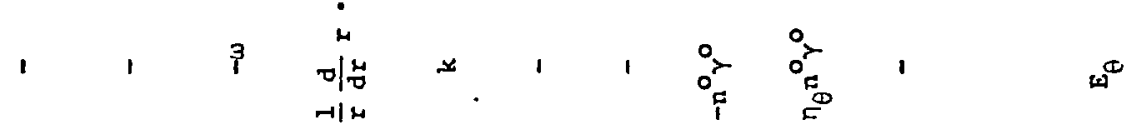

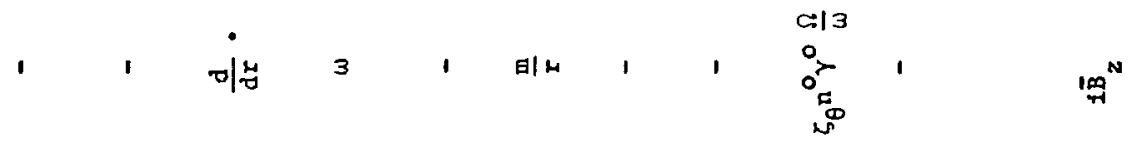

$$
\begin{aligned}
& \text { F }
\end{aligned}
$$

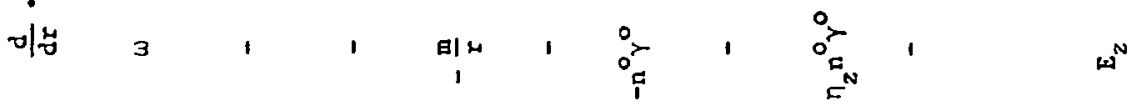



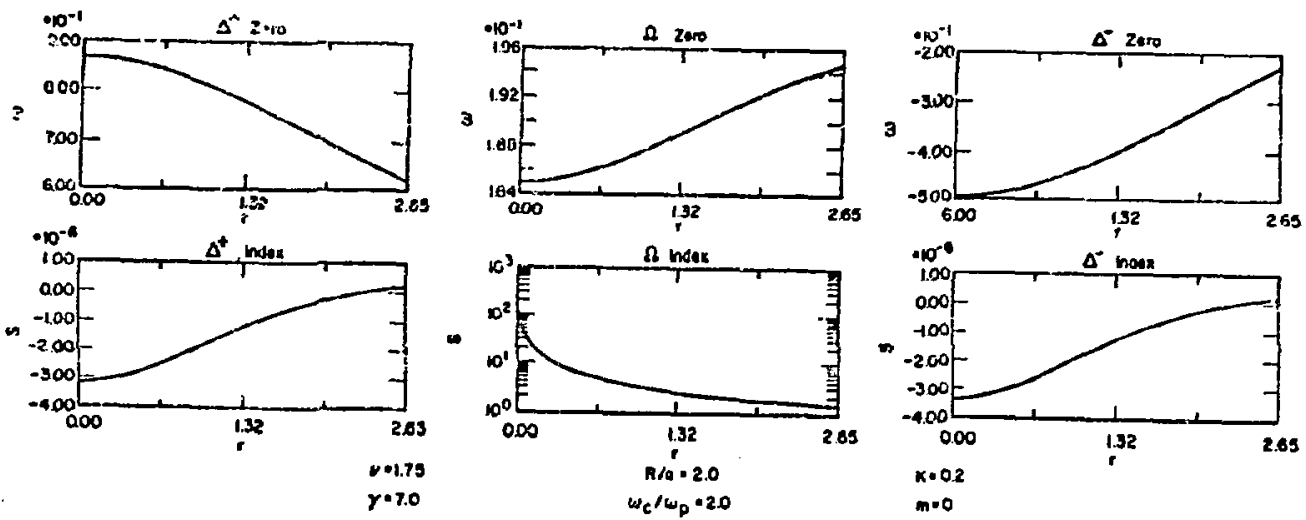

Fig. 1. For the solid beam equilibrium of Fig. 2, top row shows frequenctes corresponding to singular points of a given radius. Bottom row gives solutions to the corresponding indiclal equations. Note that the imaginary part of the $\Omega$ index is given; the real part approximates $1 / 2$. The two $\Delta$ indices are purely real.

BEAM EQUILIBRIUM DATA
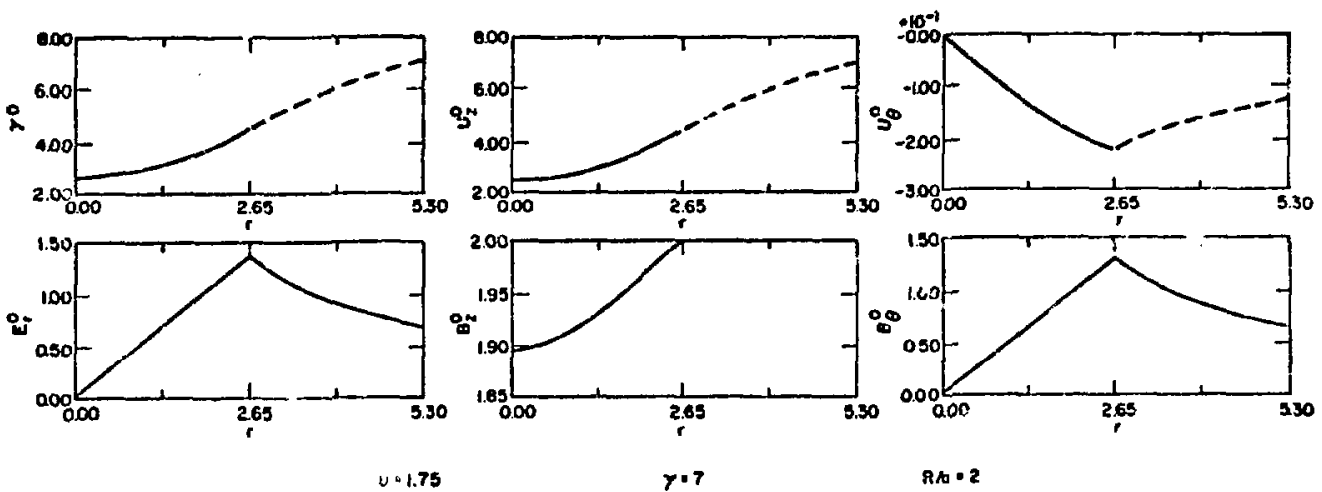

Fig. 2. Plots of various equilibrium radial profiles for a $3 \mathrm{MeV}, 30 \mathrm{kA}$ electron beam. Beam and waveguide radii are 2.65 and 5.30 , respectively. Length and time scales are $c / \omega_{p}$ and $1 / \omega_{p}$, with $\omega_{p}$ the bean plasma frequency. 


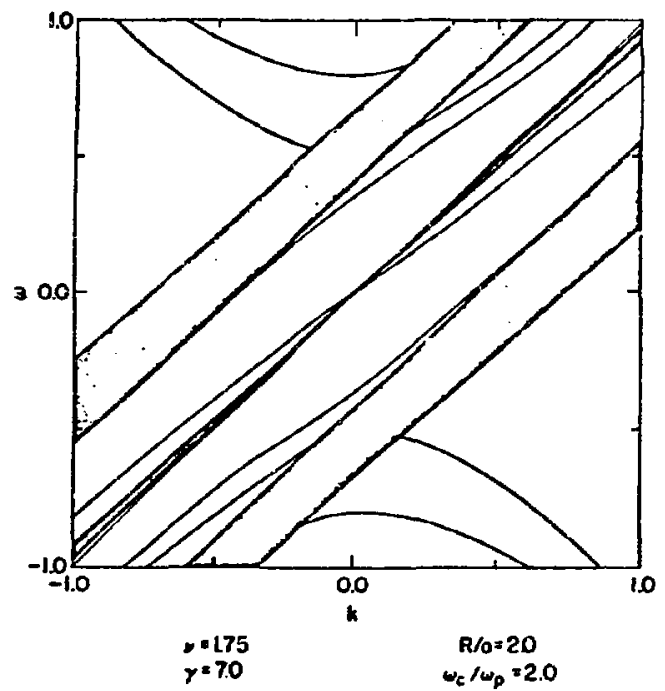

Fig. 3. Frequency versus wavenumber for axisymmetric modes in the beam equilibrium of Fig. 2, Curves represent discrete modes, while shaded areas represent resonance bands.

SLOW CrCLOTRON WAVE
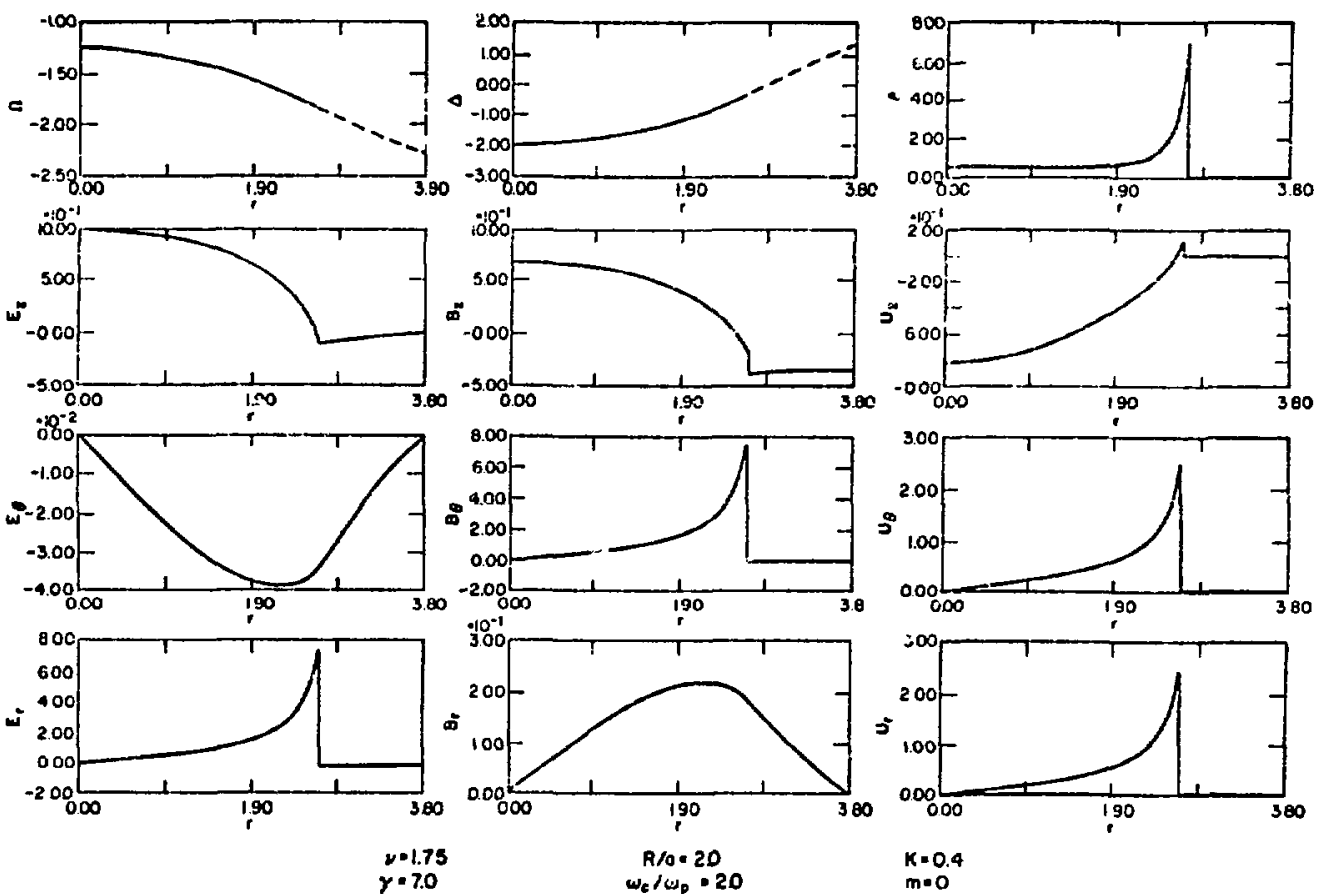

Fig. 4. Plots of perturbed particle and field quantities and of the spacecharge and cyclotron resonance functions for an $\mathrm{m}=0, \mathrm{ka}=1.06$ slow cyclotron wave. Beam equilibrium is similar to that of $\mathrm{Flg} .2$, but with $\mathrm{R} / \mathrm{a}=1.43$. 
SLOW SPACECHARGE WAVE
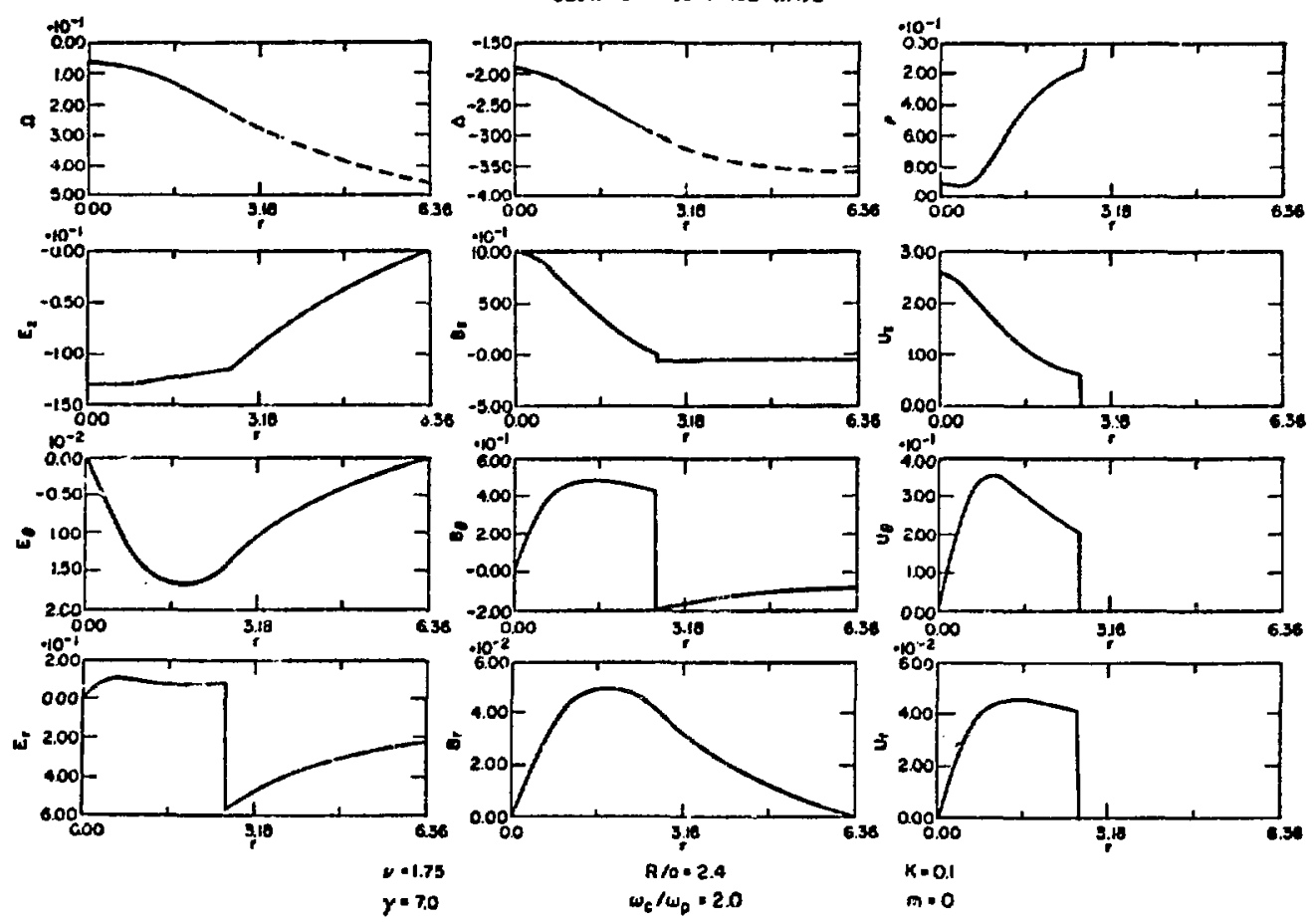

Fig. 5. Plots of perturbed particle and field quantities and of the spacecharge and cyclotron resonance functions for an $m=0, k a=0.26$ fundamental slow Langmuir wave. Beam equilibrfum is similar to that of Fig. 2, but with $\mathrm{R} / \mathrm{a}=2.40$, very near the spacecharge 1 imit.

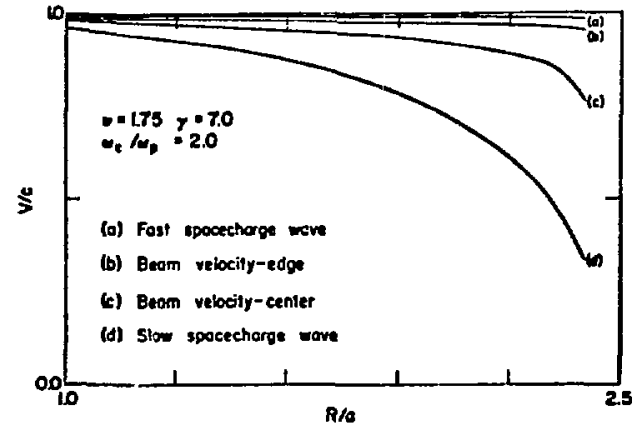

Fig. 6. Phase velocities of the long wavelength fundamental fast and slow Langmuir waves and particle velocities at the beam edge and center as functions of drift tube radius. The bean, with equilibrium similas to that of Fig. 2, becomes spacecharge limited at $\mathrm{R} / \mathrm{a}=2.4002$. 
[Reprinted from IEEE Transactions on Nuclear Science 24, 1637-1639 (1977).]

\author{
APPENDIX F \\ COMPUTER STMULATION OF COLLECTIVE ION ACCELERATION BY \\ DISCRETE CYCLOTRON MODES
}


IEEE Transactions on Nuclear Science, Vol. N'S-24, No.3, June 1977

COMPUTER SUMULATION OF COLLECTIVE ION ACCELERATION BY

\author{
DISCRETE CYCLOTRON MODES \\ R. J. Faehl, B. B. Godfrey, B. S. Newberger, W. R. Shanahan, \\ and $L$. E. Thode \\ Theoretical Diviston \\ University of Californla \\ Los Alamos Scientific Laboratory \\ Los Alamos, N. is. 87545
}

\section{Absatract}

Extensive analytical studies suggest that significant currents of high energy,ions can be obtained by collcctive acceleration $v$ ia large amplitude cyclotron waves in a non-neutral intense relativistic electron beam. We have alreidy demonstrated this acceleration mechanism in fully self-consistent two-dimensional cowputer sinulations for low lon current and energy. However, the siaulations exployed a packet of cyclotron waves created ad hoc upstreas of the acceleration region. ficceleration was limited by phase-mixing and damping of the packet. Here, we shall present results of our ongoing effort to simulate, first, realistic growth of large amplitude, single frejiency cyclotron waves in the relativistic electrcn ber.s and, second, acceieration of various lon curtents with those waves.

\section{Introduction}

Lt Il ization of intense relativistic electron beams to accelerate significant current of fons to high energy has been proposed by a number of researchers recent $1 y^{1-5}$ one of the most prosising of these schemes, advanced by Sloan and Drumbond, 5 is the Autoresonant Accelerator (ARA), involving self-consistent Browth of discrete cyclotron waves in intense relativistic electron teams with subseguent trapping of lons in their potential walls. Net acceleration occurs then by incleasing the phase velocity of the wave. There are several advantages of such.a concept over convertional ion accelezators. First, relativistic electron beam energy in the range 1-10 MeV is readily available. The envisioned accelerating fields from such beams are of the order $10^{5}-10^{6} \mathrm{v} / \mathrm{cm}$. The cyclotron wave responsible for che ficlds is, moreover, a normal mode of the bnan, it th well understood propagation characteristics. Ions tzapped in the wave potential well are assured of phase synchronism with the flelds for long perlods of time. Finelly, the linear dispersion for the dopplershifted slow cyclotron mode

$$
w=k v_{0}-\frac{\Omega_{0}}{r_{0}} \frac{\left(k v_{0}\right)^{2}}{\left(k v_{0}\right)^{2}+\omega_{p}^{2}}
$$

where $k$ is the wavenutiber, $v_{0}$ the beem velugity, $\Omega_{0}=$ $\frac{e_{a}}{m_{e}}$ the cyclotion frequency, $\omega_{p}^{2}=\left(\frac{4 c_{0} e^{2}}{m^{-1 / 2}}\right)^{1 / 2}$ the local plasma freriency, and $Y_{0}=\left(1-\left(\frac{V_{0}}{c}\right)^{2}\right)^{-1 / 2}$ indicates that it is negative energy, and so is susceptible to exponan.. tial amplification under certisin contitions. On the other fiand, the properties of large anplitude cyclotron waves, IncIuding propagation, stability, and sensitivity to nonideal bear states, are relarively unknown. Snalytic study of these questions has been undertaken, but the validtey of such models is mproven. To halp resolve questions about the nonlinear wave properties, we have conducted a combined analytic and numerical investigation of the self-consistent electron beam/cyclotron wave systers.
Numerical studies have been conducted prinarily on a fuliy relativistic, electromagnetic, 2-dinensiond, variable geonetry particle simulation code, CCube? This code is unique in its generality and ilexibility. As such, it is well sulted for study of not only the ARA scheme, but aiso such proposals as the lccalized pinch model (LPM) ${ }^{2}$ and the traveling $V$ irtual cathode accelerator. 4 These studies will be reported elsewhere

\section{Linear Dispersion in Radially Inhorogeneous Beams}

The usual procedure for finding a dispersion relation in an eluctron beam is to linearize the cold fluids equations 7,5

$$
\begin{aligned}
& \frac{\partial n}{\partial t}=-\nabla \cdot n \vec{V} \\
& \frac{\partial \gamma V}{\partial t}=-\bar{v} \cdot \nabla_{\gamma} \bar{V}+\frac{q}{m}\left(\bar{E}+\frac{\bar{v}}{c} \times \bar{B}\right)
\end{aligned}
$$

and Maxwell's equations

$$
\begin{aligned}
& \nabla \cdot \bar{E}=-4 \pi e n \\
& \nabla \cdot \bar{B}=0 \\
& \nabla \times \bar{E}=-\frac{1}{C} \frac{\partial \bar{B}}{\partial \bar{C}} \\
& \nabla \times \vec{B}=\frac{4 \pi}{C} \bar{J}+\frac{1}{c} \frac{\partial \bar{E}}{\partial t}
\end{aligned}
$$

around an electron bean equilibriur. Tho of the ccamonly employed equilibriums are the so-called "rig'drotor $^{10,8}$ with $\omega_{e}=\frac{v_{0}}{r}=$ constant and the radiaily uniform density model. Such ideaiized models are very useful for deriving analytic expressions. With uniform density, for instance, the radial cependerice of the perturbed quanities is expretsed in ter:.s of situle Bessel functions. Further, the set of discreie nodes is infinite and complete.

\section{Simulation of Io. icre]eration}

Computer simulation of lectron beam propagation in wavegtides has revealed that, although the simpie medels for equilibriua mentiened above describe the numerical results fairly well, the discrepancies can be significant. Shear in the bean kinetic energy, for instance, is an inevitable consequence of space charge eifects, but is ustally neglected when constructing analytic models. To examine such phenonena in more detail, we devised a numerical dispersion solver which inclujed radially self-consisteni jean equilibria. The results were somewhat sutprising. Kadial inlicsogeneity in the background conditions led to branch cuts in the dispersion relution, wich converts rost discrete iyclotron modes i: to contioutm, secularly decaying perturbations. To be surk, at least ore discrete mode vias always observed, bat it is not always clear a priori 
Where that node would 11e. Flgure 1 shows the 8 normal mojes of a cold, homogereous bean as solid lines. Figu:e 2 shows that the actual radial structure of a typical cyclotron mode can have far frow simple Bessel function dispendence.

To test $c$ ? e stabllicy of the wave during trapping itself, a sphericil ( $\rho-\theta)$, 2-ditenstona: particle sinulation was conducted. The relativistlc electron beam, $Y_{0}=4.5$, was infected fron the anude boundary

$(\stackrel{\omega}{c} p, \rho=100)$ and allowed to propagate to $\frac{\omega}{c} p=200$.

The beam was artificially modulated as if sas injected to give a cyclotron wave with $\frac{Q}{w_{p}}=023, \frac{\mathrm{kc}_{\mathrm{p}}}{\omega_{\mathrm{p}}}=0.46$.

The amplitude of this launched wave was large but fixed, wich a peak potential of el $\approx 1$ liev. No self-consistent growth of the wave was purported. The calculation was intended only to study the stability of a nonlinear cyclotrun wave, loaded with a nominal number of lons $\left(n_{4} / n \leq 10^{-3}\right)$, and propagating in an inhonozoneous magneric field. As figure 3, a schematic depiction of the sinulation geometry, shows, the transvecse dimension of the waveguide, and so the magnet ic field, ildred in this cunical sirulation as a function of distance fron the anode. The divergence half-angle, $\theta=.016 \mathrm{rat}$, was chosen so that this transverse dimension would double over the length of the tube. The macnetic fleld strength thus decreased by a factor of 4. Examination of the slow cyclotron dispersion relation, Eq (1), silows that the phase velocity $v_{p h}=\frac{\omega}{k}$, continually increases as the wave prupatales uuwa tite waveguide. For these pacateters, the phase velocity should have increased from $v_{\text {a }}=.05 \mathrm{c}$ to $v=.16 \mathrm{c}$, a 10-fold increase in the trapped ion energy if they remaia trâpped.

The simulation results were that once the ions were loaded irlto the potential wells, they remained stably trapped. The rave itself showed no destructive iistability as it was accelerated, altitoublit it secised to phase mix near tie end of waveguide. Careful zalysis of the simulation and comparison with numerical dispersiun solutions indicates that the exclced wave may have been a continuum, not discrete, wave, and so would be expected to decay secularly. It was otherwise similar enough to the discrete wave, that this latter's stabilicy may also be presuned. The measured ion energy was found to be amplified by a factor of almost 10 . These $\hat{i}$ igures are nodest, but they indicate the large amplitude cyclotron waves containing trapped ions can be accelerated without violent disruption of the waves. Some of the numerical results are shown in Figure 4, which has been replotted onto a rectangular brid fo: convenience of illustration.

\section{Shoath Helix Growth Hechanism}

Experimentally, it will not be as easy to cxcite a wave with predetermined characteristics as in the artificial technique described above. The wave must be self-consistently grown, extracting energy dircetly fron the team. The datails of the wave growth mechanism sust therefore be understood quantitatively. One of the wost attractive techniqueg being constutered at present involves a reactive sheath linur and a resonance instabilicy between slow electrona netic waveguide moles and the slow cyclotron waves. The use of a holical liner is the basis for the well understood craveling wave tube ${ }^{9}$, us -4 in broad- ilc: amplification with electron beams. Iir those applic, iuns, though, the beam wave is a longitudinal Langmar wave. Interaction with the cyclotron wave dors not seem to have recelved any prior attention. As a sist step, then, we have employed our cadially Inlomogeneous root solyer, with a sheath belix, to insest fyite this instability.

Typical configuration parameters for rouctively greding the cyclotron vave are triat radlus $\frac{w_{p}}{c} \mathrm{P}_{\mathrm{b}} 2.65$, cheath radius $\rightarrow p R_{0}=3.2$, and waveguide radilis of $\stackrel{p}{c} R_{0}=4.8$. The dispersion of the gufded electromagnetic waves is related to the helical pitch angle, $\psi$, approximately as

$$
\omega= \pm k \nabla \sin \psi
$$

When the frequency curves, Eqs (1) and (4), Incersect, instability occurs, leading to simultaneous growth of the posit Ive energy electromagnet ic wave and the negative energy cyclotion wave. Figs, 5 and 6 whow the frequency and associated growth race curves for the above parameter with $\psi=0.26 \mathrm{rad}, Y_{0}=7, \nu=1.75$, as a function of wavenumber, kc/w." According to these simple arguments we expect the instabilicy to be peaked in the vicinity of

$$
k=\frac{\Omega_{0}}{\gamma_{Q}\left(\beta_{0}-\sin \psi\right)}
$$

As Figure 6 indicates the numerical growth peak is at. $k c / d=.323$. Full numerical simulation of the wave grbwth process to test the validity of analyelc grouth expressions and to observe saturation mechanisms have only recently commenced. Results of these calculations should be useful in decermining the viability of the ARA concept as a practical ion acielerator, and will. be presented as a later dats.

In sumnary, we have studied the Iinear and poninear properties of the slow cyclotron wave in a relativistic electron bean with various numerical tools. We have found that modest acceleration of a small number of ions did not lead to any deleterious results. Mare exact modeling of the 1 inear cyclotron wave has showed branch cuts in the disperston relation. These lead to secularly decaying waves where discrete modes had beet expected. Since excitation of such a mode would be unsuitable for accelerator applications, it is clearly limportant to understand which modes are discrete under given conditions. Numerical studies of self-consistent growth and saturation of the slow cyclotron wave are beginning and will be reported in the Euture.

\section{References}

I. C. L. Olson, Phys. Fluids 18, 585, 598 (1975).

2. S. Putnam, Phys. Rev. Lett. 25, 1129 (1970).

3. P. Sprangle, A. T. Drobor, and W. M. Ninnheimer, Phys. Rev. Lett. 35, 1180 (1976).

4. R. B. Killer, AFWL-DYS-TN-7S-115 (Air. Force keagon Laboratory, Albuquerque, 1975).

5. M. L. Sloan and W. E. Drummond, Phys. Rev. Lett. 31, 1234 (1973).

6. B. B. Godfrey, J, Comp. Phys. 19, 58 (1975).

7. R. C. Davidson, Thenry of Nonneutral Plasmas (Benjamin, Reading, Mass.)

8. A. J. Thefss, R. A. Mahaffey, and A.k. Trivelpfece, Phys. Rev. lete. 35, 1436, 1975.

9. J. R. Plerce, Traveling Wave Tubes (D. Van kostrand, Princeton, 1950). 


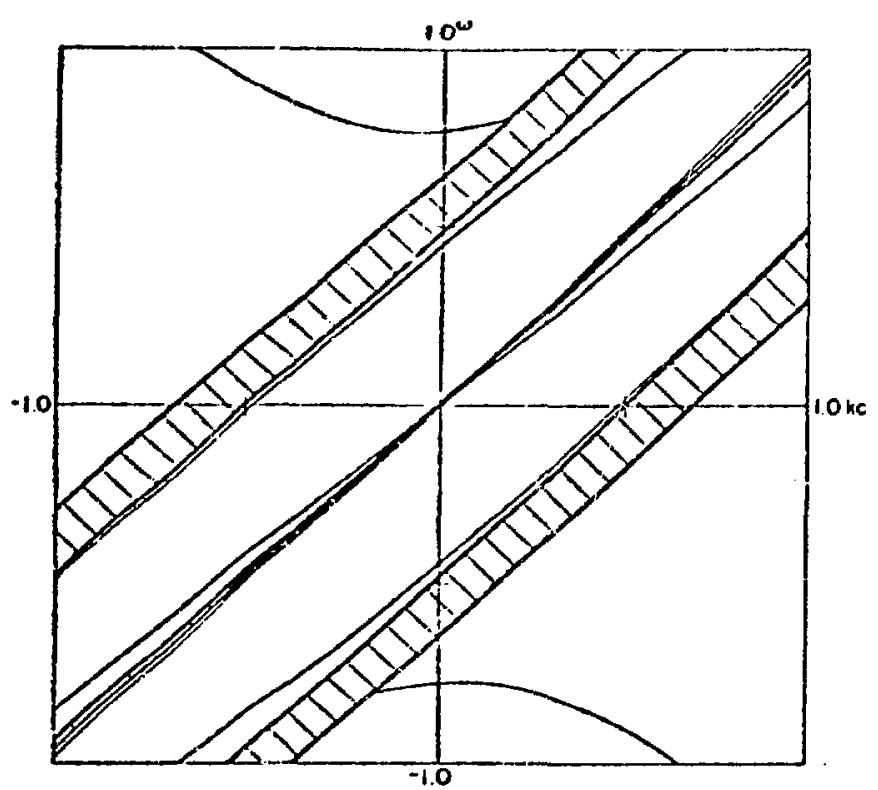

Fig. 1. Dispersica rclation, $\omega / \omega$ versus $k c / \omega$ for waves in a cj-lindsical olcctron bcam, $P_{\nu}=1 . Y_{0}=5,{ }^{P} w_{c} / \omega_{3}=2.0$, $\omega_{p} R_{b} / c=2.0$. $\omega_{0} / c=2.4$, waveguide wall grounded. I3rarch cits are denoted by cross-hatched regions (c. f. Fig. 2 of Ref. 5).

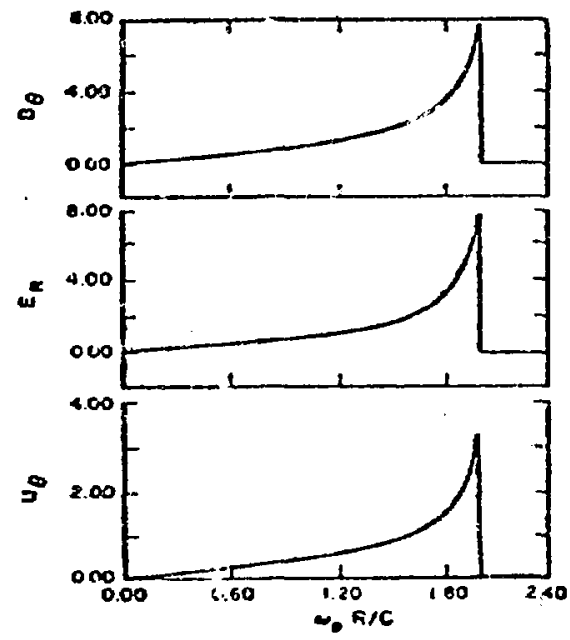

Fig. 2. Radis? structure of perturbed

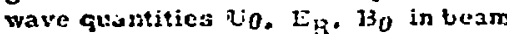
with ${ }_{\nu}=1 . \gamma_{0}=5 . \omega_{c} / \omega_{p}=2.0, \omega_{p} R$ $\omega_{0} l_{b} / c=2.0 . \omega_{p} R_{0} f_{c}=2.4, k v / w_{p}$ $=0.5 . \mathrm{m}=0$. (R nit.s are arbitrary for these perturbations.
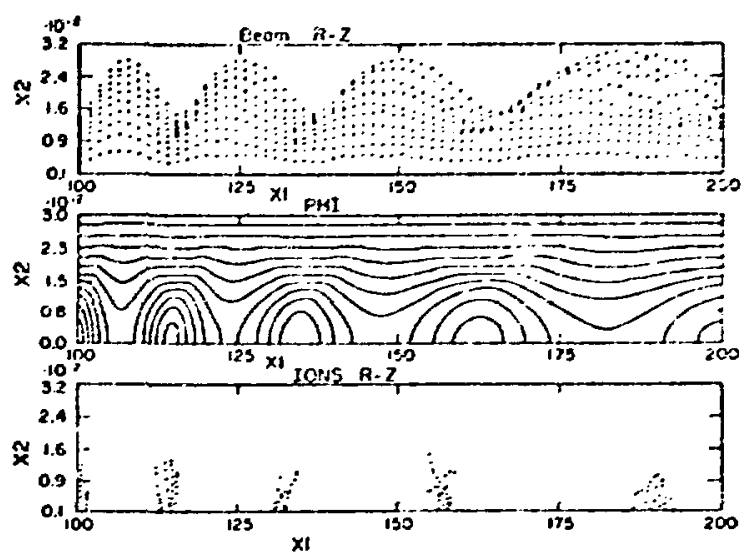

Fig. 4. Data of simulation of $v=1, y_{0}=5, w_{c} / w_{p}=2$ modulated electron beam with ions proparatine da a conical wayeguide with open ends. plotz sliow (a) electron configuration space $(X 1=z, X 2=R):(L)$ electrostatic fleld cquipotentials; (c) in corfiguration space $(\times 1-\times 2 ;$.
Fig. 3. Nominal experimental configuration correspording to the simulations shown in Fig. 4. Arrows show diverging magnetic field lires. The electron beam, occupying the shaced area, propagates from left to right. Dimensions are in $\mathrm{cm}$.
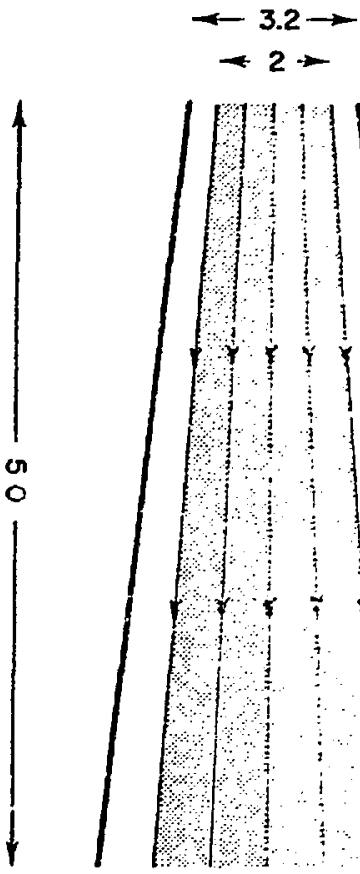

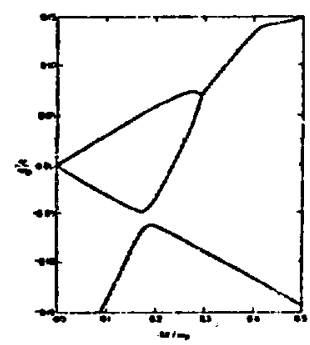

Fug. 5. Dispersion curves strowing the interscction of the doppler-shifted slow cyclotron wave with the positive frequency wavecuide mode, in a sheath helix line:. Although the dispersion curves scparate at $\mathrm{kc} / \mathrm{c}$, $=0.44$. close proximity to the cyclotron branch cut has inhibited resolution of that curve: $\nu=1.75 . \gamma_{a}=7.0 . \omega_{c} / \omega_{p}=1.65, \omega_{p}{ }^{I} b / c$

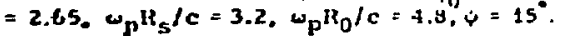

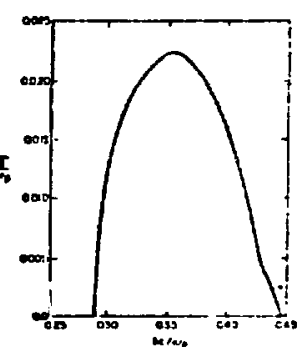

Fig. 6. Growth rate cirve for sime paraincelers as in $\mathrm{Fin}_{\mathrm{i}} .5$. 
[To be published in the Praceeaings of the Second International Topical Conference on High Power Electro: and Ion Beam Research and Technology, Cornell University, 1977.]

APPENDIX G

RELATIVISTIC ELECTRON BEAM CYCLOTRON WAVE GROWTH IN HELICAL SLOW WAVE STRUCTURES 
RELATIVISTIC ELECTRON BEAM CYCLOTRON WAVE GROITH IN HELICAL SISOW WAVE STRUCTURES*

B. B. Godfrey, R. J. Faeh1, B. S. Newberger, W. R. Shanahan, and L. E. Thode

One of the more promising and thoroughly studied proposals for high energy collective ion acceleration is Autoresonant Acceleration: Ions trapped in the electrostatic wells of large amplitude slow cyclotron waves in an unneutralized intense relativistic electron beam are accelerated by the increase of wave phase velocity as the bean propagates along a magnetic guide field of decreasing strength. A critical component of this scheme is growth of coherent, large amplitude waves. Here, we consider wave growth by interaction of the bean with a helical slow wave structure. Specific topics include (1) equilibrium charge and current distributions on the helix, (2) linear wave growth spectra including the effects of radial inhomogeneity, (3) wave growth and saturation, and (4) extraction of cyclotron waves from the amplifier cavity. Movies of two-dimensional computer simulations are presented. We find that waves of amplitude adequate for planned feasibility experiments can indeed be obtained.

\section{INTRODUCTION}

Collective ion acceleration is a highly speculative, yet potentially very significant, application for intense relativistic electron beams. Eventually, it may permit the compact and economical acceleration of substantial currents of light or heavy ions to hundreds of $\mathrm{MeV}$ per nucleon. All collective 
acceleration schemes presently envisioned involve three key stages. Slowly moving large amplitude electrostatic potential wells are established in an intense relativistic electron beam, an adequate number of ions are trapped in those we11s, and the wells with trapped ions are smoothly accelerated to velocities approaching that of the beam. Obvious sources for such potential wells are the large charge gradient at the head of an electron beam and large amplitude Langmuir or cyclotron waves in the body of the beam. 1

One of the more promising and thoroughly studied high energy collective ion acceleration schemes is the Autoresonant Acceleration proposal by Sloan and Drummond. ${ }^{2}$ It employs the slow cyclotron wave in an unneutralized intense relativistic electron beam propagating in vacuum along a strong axial magnetic field. Control of the wave phase velocity is achieved through spatial variation of the guide magnetic field. Austin Research Associates; Inc. is soon to begin an experimental investigation of this concept. 3 A $30 \mathrm{kA}, 3 \mathrm{MeV}, 200 \mathrm{~ns}$ electron beam will be used. Present plans call for the cyclotron waves to be grown in a slow wave structure at $3.4 \mathrm{~kg}$. The beam is then adiabatically compressed in a field increasing to $25 \mathrm{~kg}$, and ions 1oaded. Subsequent decrease of the axial field to $2.5 \mathrm{~kg}$ in the acceleration section should yield $30 \mathrm{MeV}$ ions.

For a successful experiment, the wave growth section must produce reasonably monochromatic, large amplitude, axially symmetric slow cyclotron waves while avoiding competing, disruptive instabilities. We at Los Alamos Scientific Laboratory are studying both analytically and computationally wave 
amplification in a sheath helix slow wave structure, one of two active candidates for the planned experiment. Our results, although not yet complete, are encouraging.

Section II describes the problem of establishing equilibrium between the beam and the helix. This is important, because the helix, being both capacitive and inductive, can support large transients lasting throughout the beam pulse. We show, however, that a proper combination of resistive termination of the helix and shaping of the beam pulse reduces transients to an innocuous level. Return currents in the helix, which disrupt the externally applied magnetic guide field, probably can be treated in the same way.

Because the slow cyclotron wave is of negative energy, while the helix-supported wave is of positive energy, they interact to produce a moderately rapidly growing convective instability which is to be responsible for cyclotron wave amplification. Section III gives the linear theory of this instability, obtained numerically from GRADR, a dispersion relation solver for radially inhomogeneous, cylindrically symmetric, cold fluid beam. equilibria. ${ }^{4}$ Analytic approximations are also provided.

Based on the linear theory, two-dimension computer simulations in cylindrical geometry have been performed with CCUBE. 5 Simulations show that, for a $2.65 \mathrm{~cm}$ radius beam in a $15^{\circ}$ pitch helix at $3.8 \mathrm{~cm}$ both enclosed in a metal waveguide at $5.7 \mathrm{~cm}$, cyclotron waves grow from a fractional modulation of $1 / 2 \%$ to about $40 \%$ is just more than a meter distance. Growth stops only when the waves leave the helix or electrons actual1y strike the helix. Wave growth obeys linear theory well, 
with little sign of nonlineai effects even at large amplitude. A11 this appears in Section IV.

Section $V$ discusses extraction of the large amplitude cyclotron waves from the helical amplifier. To date, our investigation has been limited to comparison of the linearized eigenmodes in the helix and in a waveguide of equal radius. Although frequency and wavenumber are essentially unchanged, the radial profiles of the wave components are significantly modified. The impact of this mismatch remains to be seen. II . BEAM-HELIX EQUILIBRIUM

Fig. 1 shows the relativistic electron beam just entering the helix. The smali plus signs at the helix and outer conducting waveguide represent image charges induced by the beam. Placement of these plus signs is meant to illustrate a key feature of the early time beam-helix interaction. Image charges on the helix move at a velocity

$$
\begin{aligned}
v_{H} & =\frac{c \sin \psi}{\left[1-\left(1+\frac{1-\alpha}{\ell n \alpha}\right) \cos ^{2} \psi\right]^{1 / 2}}, \\
\alpha & \equiv\left(R_{H} / R_{W}\right)^{2}<1,
\end{aligned}
$$

which for our purposes is somewhat less than the beam velocity, $v_{B} \approx c$. For example, with the parameters cited in the Introduction, $v_{H} \approx 0.3 \mathrm{c}$. Therefore, even though both ends of the helix may be grounded, the head of the beam soon outruns image charges on the helix and sees the outer conductor as the ground plane. For the experimental beam parameters, space charge effects 1 imit $R_{W}$ to no greater than $2.4 R_{B}$ for a fast beam risetime. The $80 \mathrm{~ns}$ risetime contemplated in the Austin Research Associates, Inc. experiment will, of course, substantially weaken this constraint. 
of greater importance is the fact that, because the beam reaches the far end of the helix before helix image charges do, additional images charges begin flowing onto the helix. The two streams of image charge from each end do not interact when they meet near the center but simply flow on to the helix ends, where they reflect, and so continue to stream back and forth indefinitely. Our simulations have shown this behavior to disrupt seriously the electron beam, as might be expected.

Image charge flow can be described reasonably well by the wave equation

$$
\frac{\partial^{2} \rho}{\partial t^{2}}-v_{H}^{2} \frac{\partial^{2} \rho}{\partial z^{2}}=0,
$$

with the boundary conditions $\rho+\rho_{0}=0$ at either end, $Z=0$ and $\mathrm{L}$. Here, $p$ and $\rho_{0}$ are the charge per unit length on the holix and in the beam. Eq. (2) can be solved to give a recurrence relation for, e.g., charge flowing to the right at the right boundary.

$$
\rho_{+}(t, L)=\rho_{+}\left(t-2 L / v_{H}, L\right)-\rho_{0}\left(t-L / v_{H}\right)-\rho_{0}\left(t-2 L / v_{H}-L / v_{B}\right) .
$$

Beam charge is parameterized by the time it entered the helix at the left.

The asymptotic solution to Eq. (3) is

$$
\begin{aligned}
& \rho_{+}(\omega=0)=-\rho_{0}\left(1+v_{H} / v_{B}\right) / 2, \\
& \rho_{+}\left(\omega=\pi v_{H} / n L\right)=-\rho_{0}(2 / n \pi) \omega \tau /\left(1+\omega^{2} \tau^{2}\right),
\end{aligned}
$$

for $\omega \tau \gg 1$, where $\tau$ is the characteristic rise time of the beam. Our goal, clearly, is to minimize the oscillatory part of $\rho_{+}$. For the example cited above, a helix length of one meter, and $\tau=80 \mathrm{~ns}$, the oscillatory part is of order $5 \%$. Additional reduction of transients can be achieved by placing 
resistive materials at various points in the system. With a graded resistance between the helix and the outer conductor near the downstream end of the helix, we have reduced transients in simulations to about $1 \%$ with $\omega_{1} \tau \sim 6$.

Even with oscillations in the helix image charges eliminated, a return current

$$
I_{H} / I_{B}=-\left(v_{H} / v_{B}\right)^{2}
$$

persists. This current perturbs the magnetic guide field by

$$
\Delta B_{z}=2 I_{H}(1-\alpha) /\left(R_{H} c \tan \psi\right) \text {, }
$$

or about $300 \mathrm{~g}$ for our example. The return current can be eliminated from the helix by terminating it to ground through a resistive load. Alternatively, $\Delta B_{z}$ can be externally compensated.

\section{III . LINEAR THEORY}

The helix supports a wave, $\omega=k c \sin \psi$, which interacts with the slow cyclotron wave of the beam, $\omega=\mathrm{kv}_{\mathrm{B}}-\omega_{\mathrm{C}} / \mathrm{r}$ to produce a convective instability with group velocity approximately $\mathrm{c} / 2$. The point of intersection of the two waves in $\omega$ - $k$ space is

$$
k=\omega_{c} / \gamma\left(v_{B}-c \sin \psi\right) .
$$

Austin Research Associates, Inc. has obtained an approximate analytic growth rate for a radially homogeneous beam, $\gamma \gg 1$, $k R_{B} \gg 1, R_{H}=R_{B}$, and $R_{H}=\infty .6$. The latter two constraints can be relaxed to give

$$
\Gamma=\frac{\omega_{p}}{2 \gamma}\left(\frac{\Omega_{c} R_{B}}{c} \sin 2 \psi\right)^{1 / 2}\left(e^{-2 k\left(R_{H}-R_{B}\right)}-e^{-2 k\left(R_{W}-R_{B}\right)}\right) .
$$

To improve upon these estimates, we employ GRADR, a computer code which solves Maxwell's equations together with the 
relativistic cold fiuid equations linearized about any given cylindrical beam equilibrium. ${ }^{4}$ In particular, it can treat the effects of beam energy radial inhomogenjety, $r(r)$, caused by the self-fields of the beam. Fjg. 2 presents results from this code for the parameters used throughout this article. The growth rate peaks at $\Gamma=6.8 \cdot 10^{8} \mathrm{sec}^{-1}$, corresponding to $k=0.46 \mathrm{~cm}^{-1}$ and $\omega=3.7 \cdot 10^{9} \mathrm{sec}^{-1}$. With a computed group velocity of $0.6 \mathrm{c}$, the instability growth length is $27 \mathrm{~cm}$, or about two wavelengths. Incidental1y, Eq. (7) predicts the numerically determined wavenumber precisely, if we use for $\gamma$ its value at the beam edge, 5,75. With the same choice, Eq. (8) gives a growth rate too large by nearly a factor of two. In view of the many approximations required in obtaining Eq. (8), agreement is good.

IV. WATE GROWTH AND SATURATION

In order to corroborate linear theory and, more important$1 y$, to determine the saturation level and mechanism for the beam-helix cyclotron instability, we carried out a series of two-dimensional, relativistic, electromagnecic computer simulations with CCUBE $^{5}$ configured for cylindrical coordinates. Simulations employed the beam and guide field parameters previously described but varies lengths and radii for the helix and outer conductor. Numerically well matched end conditions were used for the helix, rather than the physical terminations shown in Fig. 1, because we were interested primarily in wave growth and saturation under ideal conditions. Cyclotron waves were injected at an amplitude of $1 / 2 \%$ of the beam radius at the upstream end of the simulation grid and allowed to grow as they propagated downstream. 
For values of $R_{H}$ in the range $3.2 \mathrm{~cm}$ to $4.4 \mathrm{~cm}$, cyclotron waves grew spatially (as measured by modulation of the beam radius and by field strengths at the helix) with a growth length approximating that predicted by GRADR until either beam particles struck the helix or waves left the downstream end of the grid. Well depth increased approximately logarithmically with modulation amplitude. ${ }^{7}$ we expect that saturation by particle trapping would occur for sufficiently large helix radius; however, cost and time constraints prevent pursuing this issue.

These simulations suggest that helix and conductor radii of $3.8 \mathrm{~cm}$ and $5.7 \mathrm{~cm}$ will prove suitable for the pianned experinent, and so we have used those values throughout the report in numerical examples. With these radii the beam wave strikes the helix after about $4.5 \mathrm{e}$-foldings, or $120 \mathrm{~cm}$. The helix should, therefore, be terminated at $100 \mathrm{~cm}$, giving a potential well depth of $0.5 \mathrm{MeV}$. Maximum acceleration field strength on axis is $120 \mathrm{keV} / \mathrm{cm}$, which is more than adequate. Corresponding electric field strength at the helix is about $1 / 3$ that amount and should not cause breakdown problems. Fig. 3 shows wave growth and saturation in a $160 \mathrm{~cm}$ system. V. WAVE EXTRACTION

Fig. 4 contrasts the radial structure of characteristic components of the linearized cyclotron eigenmode, determined by GRADR, in the helix and in a metal cylinder of equal radius. Frequency and wavenumber correspond to the maximum growth rate from Fig. 2, and, with frequency fixed, wavenumber is essentially identical for the two geometries. If the disparity in radial structure persists in the nonlinear regime, 
as is likely, a real danger exists that wave coherence may be lost when the cyclotron waves exit the helix amplifier. In a few months, we hope to begin simulation studies of adiabatic transitions designed to minimize any such ill effects.

VI. ACKNOWLEDGMENT

We are endebted to E. P. Cornet for his valuable comments at various stages of this research.

\section{REFERENCES}

*Research supported jointly by the Air Force Office of Scientific Research and the Energy Research and Development Administration.

1. See, e.g., papers from the 1977 Particle Accelerator Conference (Chicago, 16-18 Mar. 77), IEEE Nuc. Sci. 24, $1625-1667$ (1977).

2. M. L. Sloan and W. E. Drummond, Phys. Rev. Lett. $\underline{31}, 1234$ (1973) .

3. W. E. Trummond, et al., unpublished.

4. B. B. Godfrey, in preparation.

5. B. B. Godfrey and W. R. Shanahan, in N. G. Cooper (ed.), "Theoretical Division Annual Report, July 1975-Sept. 1976," LA-6816-PR (Los Alamos Scientific Laboratory, 1977), p. 147 .

6. W. E. Drummond, et al., AFWL-TR-75-296 (Air Force Weapons Laboratory, Albuquerque, 1976), App. 0 .

7. B. B. Godfrey, IEEE Plas. Sci., to be published. 
HELIX UPSTREAM TERHINATIOH

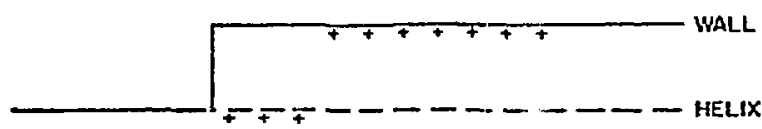

BEAM

AXIS

Fig. 1. Conceptualized transition from cylindrical wavejulde to helical amplifier cavity. Kadial dimensions are scaled to $R_{B}=2.65 \mathrm{~cm}$, $\mathrm{R}_{\mathrm{H}}=3.8 \mathrm{~cm}$, and $\mathrm{R}_{\mathrm{W}}=5.7 \mathrm{~cm}$. Small plus signs represent image charges.
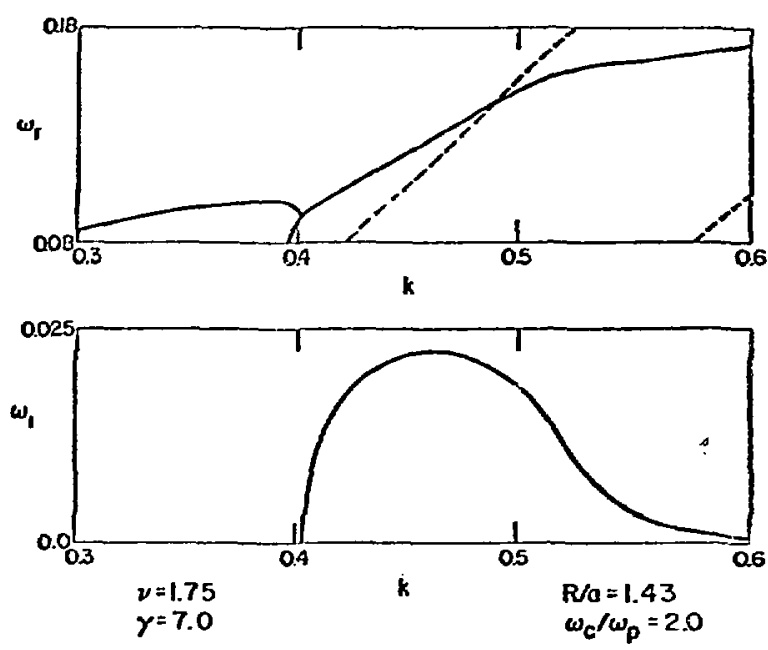

Fig. 2. Frequency and growth rate of the beamhelix cyclotron instability for a $15^{\circ}$ helix pitch as a function of wavenumber. Units are $\omega_{p}$ and $\omega_{p} / c$, respectively.
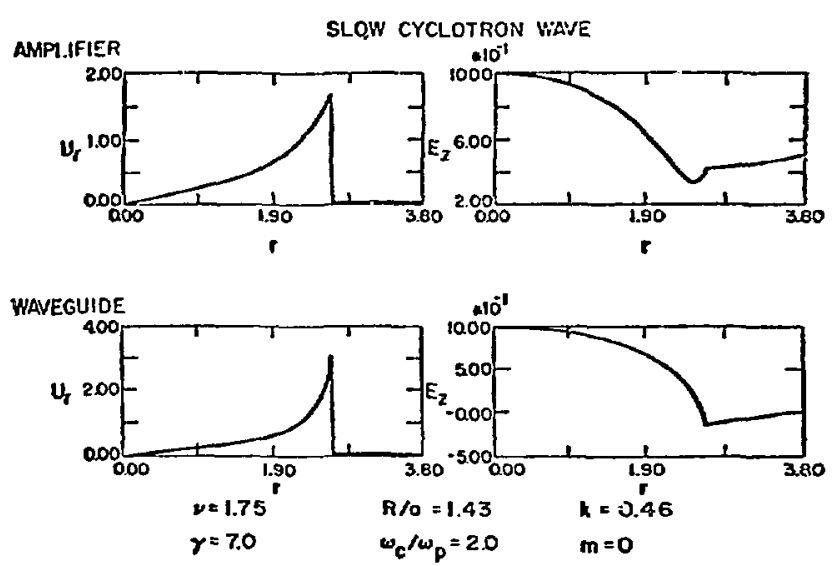

Fig. 4. Plots of perturbed radial momentum and axial electric ifeld of a slow cyclotron wave in a $15^{\circ}$ helix or in a waveguide each of radius $3.8 \mathrm{c} / \mathrm{ul}_{\mathrm{p}}$. 
[To be submitted for publication in revised form.]

APPENDIX H

SIMULATION OF CYCLOTRON WAVE GROWTH IN A HELICAL. SLOW WAVE STRUCTURE

by

R. J. Faehl, B. S. Newberger, and B. B. Godfrey 


\section{INTRODUCTION}

Large amplitude plasma waves in relativistic electron beams are an essential component of promising new collective traveling wave accelerators. 1,2 Such waves must possess both coherence and stability over long spatial and temporal scales. This is in sharp contrast to the evolution of large plasma waves in most configurations, where a rich variety of wave-wave and wave-particle interactions tends to promote damping, turbulence, solitary waves, or other nonlinear behavior. Even careful experiments with BGK modes show only short time coherence, i.e., several bounce periods. ${ }^{3-7}$ For collective ion acceleration, nevertheless, waves containing trapped ions must actually be accelerated without breaking up. In this work, we concentrate on one of the most promising electron beam modes, the slow doppler-shifted cyclotron wave, ${ }^{1}$ and study its self-consistent growth in a helical slow-wave structure.

A slow wave structure is any periodically modulated wave guide which serves to decrease the propagation speed of electromagnetic waves. The use of such guides is the basis for both conventional traveling wave accelerators $^{8}$ and traveling wave microwave amplifiers. 9 In the former, a positive energy electromagnetic wave is driven externally along a structure so designed that the fields are synchronous with the accelerating particles. The wave is stable, without other wave interactions. On the other hand, an unstable interaction of the electromagnetic wave with a negative energy electron beam mode is the key to a wide class of successful microwave amplifiers. The electromagnetic phase velocity is reduced unil it is resonant with the beam mode. Then, since the wave energies possess opposite signs, the resonance induces exponential growth in both waves. In practice, an electromagnetic wave of Iow amplitude is launched at one end of the 
waveguide, is amplified down the length of the drift tube, and extracted with another antenna at the downstream end. The quality of the associated plasma wave is of no concern, and in fact usually ends up as a broad, turbulent wave spectrum. For collective ion acceleration purposes, the electromagnetic branch is the one of no concern. It is the plasma wave we are interested in amplifying.

This study of cyclotron wave amplification combines analytic and numerical calculations to gain a better understanding of not only the wave magnitude, but also its radial structure. In this fashion, we have obtained insight into the physical mechanisms responsible for the growth and for possible limitations to growth. In Section II, we briefly review the analytic theory of the two types of waves and their unstable coupling. The deviation closely follows the more general analyses, including nonaximuthal modes, $m \neq 0$, performed by Drunmond, et a1. ${ }^{10}$ Certain simplifying assumptions are required to obtain the analytic results. In Section III, we circumvent the restrictions by numerically solving the dispersion relations on realistic beam equilibrium. One of the key results of this section is that radial inhomogeneities effect the cyclotron singularity, transforming apparent eigenmodes into secularly decaying modes. The analytic eigenmodes are found to be significantly different from the exact numerical ones. Numerical simulations were performed both to confirm the linear theory of Section III and to observe the nonlinear convective behavior of the instability. Simulations in which end effects were omitted are discussed in Section IV. In Section V, simulations including the effect of finite waveguide and heliw trminatiws are $: 1-$ cussed. Finally, the conclusions of this study are summarized and discussed in Section VI. Extrapolations to possible physical configurations are included and potential problem areas are delineated. 
II. REVIEW OF LINEAR THEORY

A periodically loaded waveguide can reduce the phase velocity of electromagnetic waves. The helix waveguide is one of the most commonly used slow wave structures. In this type of guide, a highly conducting wire is wound at a pitch angle, $\psi$, such that the direction of the wire is $\hat{t}=\cos \psi \hat{e}_{\theta}+$ $\sin \psi \hat{\mathrm{e}}_{\mathrm{z}}$. As a model for this, consider the so-called sheath helix, a cylindrical shell of infintesimal thickness, with infinite conductivity in the $\hat{\mathbf{t}}$ direction and $\sigma=0$ in any other direction. The propagation properties of this structure can be quickly sketched.

In the Lorentz gauge, the vector potentidl obeys the followirg equation In a vacuum waveguide

$$
\nabla^{2} \bar{A}-\frac{1}{c^{2}} \frac{\partial^{2} \bar{A}}{\partial t^{2}}=0
$$

If we consider a fourier component of the azimuthaliy symetric $(m=0)$ potential $\bar{A}_{k, \omega}=\bar{A}(r) e^{i k x-i \omega t}$, the $r$ and $\theta$ component equations are

$$
\frac{1}{r} \frac{\partial}{\partial r} r \frac{\partial}{\partial r} A_{j}-\frac{A_{j}}{r^{2}}+\left[\left(\frac{w}{c}\right)^{2}-k^{2} A_{j}=0\right.
$$

$\mathbf{j}=\mathbf{r}, \theta$, which has modified Bessel function solutions. For a helix at $r=R_{H}$, the requirement that the solutions are well behaved at $\mathbf{r}=0$ and $\mathbf{r}=\infty$ are

$$
A_{j} \propto \begin{array}{ll}
K_{i}(q r) & r>R_{H} \\
I_{1}(q r) & r<R_{H}
\end{array}
$$

where $q=\left(k^{2}-\left(\frac{\omega}{c}\right)^{2}\right)^{1 / 2}$. The $z$-component is derived from the Lorentz condition, $\frac{1}{c} \frac{\partial \phi}{\partial t}+\nabla \cdot \bar{A}=0, \phi \cong A_{z} \cdot$ The fields themselves are easily caiculated from the potentials. 
Thus when $r>R_{H}$

$$
\begin{aligned}
& A_{r}=\alpha K_{1}(q r) \\
& A_{\theta}=B K_{1}(q r) \\
& A_{z}=i \frac{q \alpha K_{0}(q r)}{\left(\frac{\omega}{c}-k\right)}
\end{aligned}
$$

so that

$$
\begin{aligned}
& B_{r}=-i k \beta K_{1} \\
& B_{\theta}=-i \frac{\omega}{c} \alpha K_{1} \\
& B_{z}=-q \beta K_{0} \\
& E_{r}=i\left(2 \frac{\omega}{c}+k\right) \alpha K_{1} \\
& E_{\theta}=i \frac{\omega}{c} \beta K_{1} \\
& E_{z}=-q \alpha K_{0} .
\end{aligned}
$$

Simflarly when $r<R_{A}$

$$
\begin{aligned}
& A_{r}=\gamma I_{1}(q r) \\
& A_{\theta}=\delta I_{1}(q r) \\
& A_{z}=-i \frac{q \gamma I_{0}}{\left(\frac{\omega}{c}-k\right)}
\end{aligned}
$$

and 


$$
\begin{aligned}
& B_{r}=-i k \delta I_{1} \\
& B_{\theta}=-i\left(\frac{\omega}{c}\right) \gamma I, \\
& B_{z}=q \delta I_{0} \\
& E_{r}=-i k \gamma I_{1} \\
& E_{\theta}=\frac{i \omega}{c} \delta I_{1} \\
& E_{z}=+q \gamma I_{0}
\end{aligned}
$$

The appropriate boundary conditions at $r=R_{H}$ are that

$$
\begin{aligned}
\overline{\mathrm{E}} \cdot \hat{\mathrm{t}} & =0 \\
\left.E_{\theta}\right|_{<} & =\left.E_{\theta}\right|_{>} \\
\left.E_{z}\right|_{<} & =\left.E_{z}\right|_{>} \\
\left.\bar{B} \cdot \hat{\mathrm{t}}\right|_{<} & =\left.\bar{B} \cdot \hat{\mathrm{t}}\right|_{>}
\end{aligned}
$$

where $<(>)$ signifies $r<R_{H}\left(>R_{H}\right)$. The first boundary condition simply expresses the fact that the helix is perfectly conducting, the second and third, the continuity of the tangential components of the E-field, and the fourth condition is equivalent to a statement that current can only flow along the helix at $r=R_{H}$. Application of these boundary conditions yields four equations in four unknowns, $\alpha, \beta, \gamma$, and $\delta$. Simultaneous solution of these results in the dispersion relation

$$
\omega^{2}=q^{2} c^{2} \frac{I_{o}\left(q R_{H}\right) K_{o}\left(q R_{H}\right)}{I_{1}\left(q R_{H}\right) K_{1}\left(q R_{H}\right)} \tan ^{2} \psi
$$

When a relativistic electron beam of radius $R_{B}<R_{H}$ is present, the slow waveguide mode may be able to interact resonantly with a waveguide mode. 
Formally the beam will support current, which will result in nonzero source terms, i.e., $\frac{4 \pi}{c} \bar{J}$, on the RHS of (1). To calculate these currents consider the $\mathbf{r}$ and $\theta$ components of the cold fluid plasma equations

$$
\frac{d \bar{p}_{i}}{d t}=-\frac{e}{m_{c}}\left(E_{i}+\left[\frac{\bar{v}}{c} \times \bar{B}\right]_{i}\right)
$$

where $i=r, \theta, p_{1}=\gamma_{0} v_{i}, \gamma_{0}=\left(1-\left(\frac{v}{c}\right)^{2}\right)^{-1 / 2}, B_{z}=B_{0}$. There are equilibrium motions associated with the vacuum, unneutraliced propagation of an electron beam along the $\mathrm{B}_{2}$ guide field. For $\gamma_{0} \gg 1$, however, we can show that $\frac{v_{\theta}}{r} \ll \frac{\Omega_{0}}{\gamma_{0}}, \Omega_{0}=\frac{e_{0}}{m_{e} c}$. Since the beam is in equilibrium, we take $v_{R}=0$, although a more general condition may be $\left\langle\mathrm{V}_{\mathrm{R}}\right\rangle_{\mathrm{z}}=0$, with the brackets denoting averaging in the $\mathrm{z}$-direction. Then for $\mathrm{m}=0$, neglecting diamagnetic effects and rotation, the equations are

$$
\begin{aligned}
& \frac{d p_{r}}{d t}+\frac{\Omega_{o}}{\gamma_{0}} p_{\theta}=-\frac{e}{m}\left(E_{r}-\frac{v_{o}}{c} B_{\theta}\right) \\
& \frac{d p_{\theta}}{d t}-\frac{\Omega_{o}}{\gamma_{o}} p_{r}=-\frac{e}{m}\left(E_{\theta}+\frac{v_{o}}{c} B_{r}\right)
\end{aligned}
$$

where $v_{0} \equiv v_{z}$. When $\gamma_{0} \gg 1$, we find $A_{z} \simeq \phi, \phi$ the electrostatic potential. Consequently,

$$
\begin{aligned}
& \left(E_{r}-\frac{v_{o}}{c} B_{\theta}\right)=\frac{i}{c}\left(\omega-k v_{o}\right) A_{r}+o\left(1-B_{o}\right) \\
& \left(E_{\theta}+\frac{v_{0}}{c} B_{r}\right)=\frac{1}{c}\left(\omega-k v_{o}\right) A_{\theta} .
\end{aligned}
$$

Both Eqs. (10a) and (10b) are much simplified by the change of variables 


$$
\begin{aligned}
& A^{+}=A_{r}+i A_{\theta} \\
& A^{-}=A_{r}-i A_{\theta} \\
& p^{+}=p_{r}+i p_{\theta} \\
& :^{-}=p_{r}-i p_{\theta}
\end{aligned}
$$

Equations (10a) and (10b) then become

$$
\begin{aligned}
& \frac{d^{+}}{d t}+i \frac{\Omega_{0}}{\gamma_{0}} p^{+}=\frac{e}{m c}\left(\omega-k v_{0}\right) A^{-} \\
& \frac{d p^{-}}{d t}-i \frac{\Omega_{0}}{\gamma_{0}} p^{-}=-\frac{e}{m c}\left(\omega-k v_{0}\right) A^{+}
\end{aligned}
$$

while Eq. (2) in component format is

$$
\begin{aligned}
& \left(\nabla^{2}-\frac{1}{r^{2}}+q^{2}\right) A^{+}=+\frac{4 \pi e n(r) i}{\gamma_{0} c} p^{-} \\
& \left(\nabla^{2}-\frac{1}{r^{2}}+q^{2}\right) A^{-}=-\frac{4 \pi e n(r) i}{\gamma_{0} c} p^{+} .
\end{aligned}
$$

If we let $p^{ \pm}$o $e^{i(k x-u \cdot t)}$, then the solution for the special case $n(r)=n_{0}$ is simply obtained

$$
A^{ \pm} \propto J_{1}\left(k^{ \pm} r\right)
$$

where

$$
k^{ \pm}=\left[\left(\begin{array}{l}
\omega \\
c
\end{array}\right)^{2}-k^{2}-\frac{\omega_{p}^{2}}{\gamma_{0} c^{2}}\left(\frac{\omega-k_{0}}{\omega-k_{0} \pm \frac{\Omega_{0}}{\gamma_{0}}}\right)\right]^{1 / 2}
$$


when $w \ll\left(k^{2}+\left(k^{ \pm}\right)^{2}\right)^{1 / 2} c$, this yields the dispersion relation

$$
\omega=k \nu_{0} \pm \frac{\Omega_{0}}{\gamma_{0}} \frac{\left(k^{2}+\left(k^{ \pm}\right)^{2}\right) c^{2}}{\left(k^{2}+\left(k^{ \pm}\right)^{2}\right) c^{2}+\frac{\omega_{p}^{2}}{\gamma_{0}}}
$$

We are interested in the slow-doppler-shifted cyclotron wave here, the minus root. Then note that when $\gamma \gg 1, \omega \simeq \mathrm{kv}_{0}-\frac{\Omega_{0}}{\gamma_{0}}$. Resonance of this mode with the slow electromagnetic waveguide mode occurs at approxinately

$$
k=\frac{\Omega_{0}}{\gamma_{0}} c /\left(B_{0}-\sin \psi\right)
$$

where $\beta_{0}=\frac{v_{0}}{c}$.

Application of buundary conditions at $r=R_{b}$ and linearization of (13), (14), and (7a)-(7d) can be used to find the growth rate for this instability. This analysis closely follows that of Wong, et a1. ${ }^{11}$ who have explicitly considered an arbitrary axial mode, i.e., $m \neq 0$. The expression for the growth rate is finally

$$
\Gamma \cong\left[\frac{\pi \omega_{o} \Omega_{o} q^{2}}{k^{2} c^{2}} e^{-2 q R_{B}} \int_{0}^{R_{B}} \omega_{p}^{2}(r) \operatorname{rdrI} I_{I}^{2}(r)\right]^{1 / 2}
$$

It remains to be seen whether this expression, though, is accurate in the regimes envisioned for growth of the cyclotron waves. To address that question, we have solved the dispersion relation numerically. 
III. NUMERICAL COMPUTATION OF EIGENFUNCTIONS AND GROWTH RATES

Analytic expressions were obtained in the previous section for eigenfunctions and dispersion relations of the slow electromagnetic waves and the slow cyclotron waves, Eqs. (4), (6), and (8), and (15), (16) respective1y. These expressions were derivable because we neglected equilibrium beam rotation, radial energy variations, and self-fields. The state about which we linearized was, therefore, artificial and somewhat idealized. As a result, these simple analytic forms may show significant discrepancies from the realistic functions. In particular, radial inhomogeneities can lead to a situation where different radii oscillate at different local frequencies. If the inhomogeneity is severe enough, the contributions to the total eigermode may be sufficient?y out-of-phase that phase mixing of the wave occurs. Mathematically, this is analyzed by examining the branch points of the dispersion relation. This has been done elsewhere, ${ }^{12}$ with the conclusion that the branch cut can "swallow" modes, transforming them into secularly decaying envelopes. A proof due to sioan, et al. ${ }^{10}$ shows that at least one slow cyclotron wave will persist in a cold beam, but there is no guarantee that it will possess the desired structure or propagation characteristics. We have, therefore, solved the cold-fluid, electromagnetic dispersion relation on self-consistent radial beam profils.s.

The code GRADR was used to numerically determine the eigenfunctions and dispersion relations. As it has been described in detail elsewhere, 12 we will merely sketch its methodology here. If all quantities vary as $e^{i k z+i m \theta-i \omega t}$, the cold fluid equations plus Maxwe11's equation can be written

$$
\begin{aligned}
& -i\left(\omega-\mathrm{m} \frac{v_{\theta}}{r}-k v_{z}\right) n=-n\left(\frac{1}{r} \frac{\partial}{\partial r} r v_{r}\right) \\
& -1\left(\omega-m \frac{v_{\theta}}{r}-k v_{z}\right) \bar{p}=-\frac{e}{m_{e}}\left(\bar{E}_{z}+\frac{1}{c \gamma} \bar{p} \times \bar{B}\right)
\end{aligned}
$$




$$
\begin{aligned}
1 k E_{z}+\frac{i m}{r} E_{\theta}+\frac{1}{r} \frac{\partial}{\partial r} r E_{r} & =-4 \pi e n \\
i k B_{z}+\frac{1 m}{r} B_{\theta}+\frac{1}{z} \frac{\partial}{\partial r} r B_{r} & =0 \\
-i \frac{\omega}{c} \bar{B}+\nabla \times \bar{E} & =0 \\
& +i \frac{\omega}{c} \bar{E}+\nabla \times \bar{B}=-\frac{4 \pi e}{c \gamma} n \bar{p}
\end{aligned}
$$

where $\bar{p}=\gamma \bar{v}, \gamma=\left(1-\left(\frac{v}{c}\right)^{2}\right)^{1 / 2}, v^{2}=v_{r}^{2}+v_{\theta}^{2}+v_{z}^{2}$. Linearization of (19) such that

$$
\begin{aligned}
& n=n_{0}(r)+\tilde{n} \\
& v_{r}=\tilde{v}_{r} \\
& v_{\theta}=v_{\theta}(r)+\tilde{v}_{\theta} \\
& v_{z}=v_{z}(r)+\tilde{v}_{z} \\
& \bar{E}=\tilde{E}_{0}(r)+\tilde{E} \\
& \bar{B}=\bar{B}_{0}(r)+\tilde{B}
\end{aligned}
$$

gives 10 equations, 5 algebraic and 5 differential equations, although analysis of the differential system reveals that no more than 4 of these are independent. Equilibrium is found by iterating an assumed initial radial distribution of the zero order quantities until the field, momentum, and energy profiles are seif-consistent. This equilibrium is then used in the algebraic-differential set of coupled first order equations. A fourth order Runge-Kutta scheme is used to solve the differential subset.

For these problems, we assumed that a monoenergetic, solid beam of uniform density was injested into a vacuum drift tube. Space charge effects arise in the guide tube. For the other equilibrium condition, we take 
$Y_{0}-1=\gamma-1-\frac{e \phi}{m^{2}}$ to arrive at the density and energy $(\gamma)$ profiles shown in Fig. 1, a cilculation with $\gamma_{0}=7(3 \mathrm{MeV}), R_{B}=2.65 \mathrm{c} / \omega_{\mathrm{p}}, \mathrm{R}_{\mathrm{H}}=3.2 \mathrm{c} / \omega_{\mathrm{p}}$, and $\Omega_{0}=1.65 \omega_{p}$, where $\Omega_{0}=e^{B}{ }_{z 0} / m_{e} c, R_{B}=$ radius of the electron beam, $R_{H}=$ radius of the helix and $\omega_{p}^{2}=4 \pi \bar{n} e^{2} / m_{e}$. The radius is related to Budker's current parameter,$\nu=\left(R_{B} \omega_{p} / 2 c\right)^{2}$. The helix is grounded, so it is the effective outer wall for electrostatic effects. With these parameters, the density exhibits little radial inhomogeneity. Radial energy variations are considerable, however. Figure 2 shows the equilibrium $E_{r}, B_{\theta}$, and $B_{z}$ components along with the equilibrium rotational frequency $\omega_{\theta} \equiv \frac{V_{\theta}}{r}$. I'he relativistic gyrofrequency, $\frac{\Omega_{0}}{\gamma_{0}}$, for this case is $0.24 \omega_{p}$. Sirce this is only 4 times greater than $\omega_{\theta}$, neglect of rotation is not warranted. The rotation furthermore possesses significant shear. With increasing beam voltage, the rotational Erequency, shear, and diamagnetic effects all decrease. Approximate dispersion relations vere derived in the previous section. By comparing these with numerical dispersion relations, we can estimate the error induced by the idealized equilibrium conditions.

Eq. (8) is the dispersion relation for electromagnetic helically guided waves. We omitted an external conducting wall in deriving (8). This is tantamount to assuming a boundary at $r=\infty$. Since the fitelds fall off as either $K_{0}(q r)$ or $K_{\perp}(q r)$, the presence of the wall at a finite distance will tend to decrease the magnitude of the fields. Its effect on the dispersion is less obvious. Eq. (8) also assumed that the space interior to the helix was empty. We are only interested in cases in which an unneutralized electron beam is present, however. Although the beam is not likely to alter the helix dispersion, it may change the field structure, which in turn could modify the unstable coupling. The numerical dispersion soluticns for $R_{B}=2.65$, $R_{W} / R_{H}=1.5$ and $\psi=15^{\circ}$ are indicated by the solid line in Fig. 3 . The 
dashed line by comparison represents Eq. (8) for these conditions. The analytic expression consistently overestimates the frequency, and so also, the phase velocity of the wave. The group velocity, $\frac{\partial \omega}{\partial k}$, however, is in better agreement. Interestingly, the large argument limit of (8), $\omega=k c \sin \psi$, agrees with the numerical result considerably better than ( 8 ) Itself for these parameters, even though $q R_{\mathcal{H}} \approx 1$ here.

The slow deppler-shifted cyclotron wave dispersion reiation is given by Eq. (16). For $R_{B}=2.65 \mathrm{c} / \omega_{p}, R_{w}=3.2 \mathrm{c} / \omega_{p}, \Omega_{0} / \omega_{p}=2$ and $\gamma_{0}=7$, the numerical solution is shown in Fig. 4, with the solid line. In this case we compared it with the low density, high voltage dispersion relation, $\omega=k v_{0}-\frac{\Omega_{0}}{\gamma_{0}}$ Again the analytic expression predicts too large a frequency. (The more accurate expression, (16), is always larger than the dashed line.) The effects of radial inhomogeneity are first noted here by the occurrence of a "branch cut" region just below the eigenfrequency for these conditions The close proximity of this region, shown as a shaded area in Fig. 4, is particularly interesting because the strong coupling of the waveguide and beam modes will induce a frequency shift into that region.

So far, a sample comparison of analytic with numerical results has shown quantitative but not qualitative differences. The radial structure of che fields, however, is quite dissimilar. Figure $5(a)$ shows the field structure of a helix mode with $k=0.36 \omega_{p} / c, \omega=0.1043 \omega_{p}$ and other parameters the same as in Fig. 1. As a comparison Eq. (6) is plotted along with the $E_{2}$ component of the numerical eigenfunction, normalized to the sane value at the helix in Fig. 5(b). The analytic expression is clearly in good agreement outside the beam, but quite different inside. Figure 6 shows the cyclotron eigenfields, without a helical guiding structure for parameters similar to cases in the above figures at $k=0.42 \omega_{p} / c$ and $\omega=0.116 \omega_{p}$. Comparison with analytic expressions has shown some qualitative similarities, but significant quantitative disparities. 
Finally, the stability analysis of the coupling between cyclotron and waveguide modes yielded several results which can be checked against the numerical solutions. One of the most obvious is the effect on the respective dispersion relations. The dispersion of the stable modes was shown in Figs. 3 and 4. Where those curves intersect, however, the modes couple unstably and merge into a single mode over a finite region of $k$-space. The resulting dispersion curves are illustrated in Fig. 7 , for $\Omega_{0}=1.65 \omega_{p}$, $\gamma_{0}=7, \nu=1.75, \psi=15^{\circ}$, and $R_{B}=2.65 \mathrm{c} / \omega_{p}, R_{H}=3.2 \mathrm{c} / \omega_{p}$, and $R_{W}=4.8 \mathrm{c} / \omega_{p}$. The frequency and group velocity of the unstable waves are both roughly midway between the stable waveguide and cyclotron modes, that is

$$
\omega \cong \frac{k\left(v_{0}+c \sin \psi\right)-\frac{\Omega_{0}}{\gamma_{0}}}{2}
$$

The unstable modes have therefore been shifted toward the cyclotron branch cut region. This does not affect the unstable waves as badly as might be feared, since the waveguide mode, which possesses no singularities in this region, apparently gives it a certain robustness. However, on the short wavelength side of the instability it is very difficult to find the cyclotron branch again. Thus, while the wavrguide branch is clearly distinguishable at high $k$, the branch cut has apparently destroyed any stable cyclotron waves there. This is potential: $y$ a very serious problem with regard to collective ion acceleration. The utility of a helical amplifier for cyclotron waves is greatly diminished if the wave secularly decays as soon as it leaves the amplifier. This problem is being pursued analytically and numerically at the present time, with the results to be presented in a future articie. 
The approximate analytic growth rate derived in the last section is Eq. (18), where $\omega_{0}=k v_{0}-\frac{\Omega_{0}}{\gamma_{0}} \simeq q c \tan \psi$ was assumed. For the parameters in Fig. 7, this expression is in fair agreement with the peak numericai growth rate, $\Gamma_{\max }=0.022 \omega_{\mathrm{p}}$. If the analytic expression is simple-mindedly adjusted for finite beam-helix separation and finite outer wall, one finds $\Gamma_{\text {theory }}=0.016 \omega_{p}$. More important than the magnitude though is the scaling with beam or helix parameters. Let us first consider the dependence on the guide $B_{o}$ field.

Eq. (18) apparently predicts peak growth rate scaling $\Gamma \propto \mathrm{B}_{0}^{1 / 2}$. In fact since the peak growth occurs near $k=\frac{\Omega_{0}}{\gamma_{0}} /\left(v_{0}-c \sin \psi\right)$, the term $\omega_{0}$ is approximately

$$
\omega_{0} \simeq\left(\frac{c \sin \psi}{v_{0}-c \sin \psi}\right) \frac{\Omega_{0}}{\gamma_{0}}
$$

We may therefore infer that $\Gamma_{\max } \propto \mathrm{B}_{\mathrm{O}}$ will be a more accurate scaling. (Eq. (21) is only approximate. Though a comparison with numerical values of $k$ at peak growth in Fig. 8 shows a quantitative discrepancy, $k_{\max } s t i 11$ varies 1inearly with $\mathrm{B}_{\mathrm{O}^{\circ}}$ )

Figure 9 shows the maximum growth rate as a function of $B_{0}$ for $\gamma_{0}=7$ (solid line) and $\gamma_{0}=20$ (dashed line), with $R_{B}=2.65 \mathrm{c} / \omega_{p}, R_{H}=3.2 \mathrm{c} / \omega_{p}$, $R_{W}=4.8 \mathrm{c} / \omega_{\mathrm{P}}$, and $\psi=15^{\circ}$. At both voltages, the functional dependence is more complicated than simple linearity. When the relativistic gyrofrequency is low enough, i.e., $\frac{\Omega_{0}}{\gamma_{0}} \tilde{<} 0.4 \omega_{p}$, both curves exhibit the expected innear dependence on the field. The reason for the decrease in growth when $\frac{\Omega_{0}}{\gamma_{0}} ; 0.6 \omega_{p}$ Is not clear, but since the wavelength is falrly small. $k>0.9 \omega_{p} / c$, th is possible tilat the cyclotron branch cut exerts a particularly deleterious effect on the coupling. This is also suggested by the increasingly sniked field structure. 
By the reasoning that led us to expect a linear field scaling from Eq. (18), we may suppose that the peak growth varies as $\gamma_{0}^{-3 / 2}$. Figure 10 shows curves for the maximum growth rate as a function of $\gamma_{0}$, for $\Omega_{0}=2.0 \omega_{p}$ (solid) and $\Omega_{0}=10 \omega_{p}$ (dashed line). Again when $\frac{\Omega_{0}}{\gamma_{0}}<0.4 \omega_{p}$, we find $\Gamma_{\max } \propto \gamma_{0}^{-3 / 2}$, but significant discrepancies are seen when $\frac{\Omega_{0}}{\gamma_{0}} \check{>} 0.6-0.8 \omega_{\mathrm{p}}$. (Calculations out to $\gamma_{0}=50$ confirm the $\gamma_{0}^{-3 / 2}$ scaling in the $\Omega_{0}=10 \omega_{p}$ case, but are not depicted for reasons of plotting compactness.) Furthermore, in the parameter regime in which the $\gamma_{0}^{-3 / 2}$ scaling is valid, we also find $\Gamma_{\max } \propto B_{0} . \quad$ Reference to Fig. 8 again confirms that the growth in the linear region varies as $\gamma_{0}^{-3 / 2}$.

Finally, Eq. (18) does not explicitly show any dependence on the pitch angle, $\psi$. Since $(18)$ is valid when $\omega_{0} \cong q c \tan \psi$, however, we infer that $\Gamma_{\max } \propto(\tan \psi)^{1 / 2}$. Figure 11 exhibits $\Gamma_{\max }$ as a function of $\psi$, when $\Omega_{0}=2.0 \omega_{p}, \gamma_{0}=7, R_{B}=2.65 \mathrm{c} / \omega_{p}, R_{H}=3.2 \mathrm{c} / \omega_{p}$, and $R_{T x}=4.3 \mathrm{c} / \omega_{p}$. The dashed line merely shows a $(\operatorname{tar} \psi)^{1 / 2}$ variation for comparison. The agreement is clearly excellent up to $\psi \cong 30^{\circ}$. Again it should be noted that the growth turns over at about $40^{\circ}$, where $k \sim \omega_{p} / c$. For this case, however, maximized growth at $\psi \simeq 45^{\circ}$ is not unexpected. A bett $i$ scaling when $\psi \sim 30^{\circ}$ might be $(\sin 2 \psi)^{1 / 2}$.

There is one additional piece of information of interest about the growth which we can obtain numerically, the effect of finite outer wall radius and finite beam-helix separation. An example of the former is exhibited in Fig. 12, in which parameters similar to Fig. 11 were used, except $\psi=15^{\circ}$. Since the amplitude of the cyclotron wave is roughly proportional to the beam modulation magnitude, we desire a significant separation of helix and beam to avold having the wave "hit" the helix. As we will see in the next section, beam contact with the helix effectively destroys the 
wave. However, as Fig. 11 shows, the instability growth rate decreases roughly as $P_{H} / R_{B}$. Thus, a "safe" separation distance may entafl an unacceptably long cyclotrun amplification length.

The oiner effect is finite outer wall radfus. This tends to decrease the growth rate, as the example in Fig. 13 shows. The solid 1 ine indicates the exact numerical result, the dashed line a curve varying as $\Gamma_{\text {max }}=\Gamma_{\mathrm{o}}\left(1-\mathrm{e}^{2 \mathrm{q}\left(\mathrm{R}_{\mathrm{H}}-\mathrm{R}_{\mathrm{W}}\right)}\right)$, and the dotted 1.ne, the growth scaling

$$
\Gamma_{\max }=\Gamma_{0}\left(1-\frac{K_{0}\left(q R_{W}\right) K_{1}\left(q R_{W}\right)}{K_{0}\left(q R_{H}\right) K_{1}\left(q R_{H}\right)}\right) \text {. }
$$

As expected decreajing the helix-outer wall septration weakens the instability.

Summarizing these numerical results, we find that

$$
\Gamma \propto\left(\frac{\Omega_{0}^{2}}{\gamma_{0}^{3}} \tan \psi\right)^{1 / 2} \omega_{p}
$$

as long as $\mathrm{kc} / \mathrm{w}_{\mathrm{p}} \tilde{<}$. Thus, Eq. (18) gives reasonably accurate scalings over a parameter space which is of interest to the Autoresonant Accelerator. ${ }^{1}$ Th reason for qualitative differences in the growth rate when $k c / \omega_{p} \tilde{>} 1$ has not been uncimbiguously identifled, but there are indications that it may be associated with inhomogeneity in the cyclotron singularity. 
IV. NUMERICAL SIMLATIO: OF CYCLOTRON WAVE GROWTH VIA COUPLING WITH A HELICAL LINER

In the previous sections, we considered the coupling of waveguide and cyclotron waves leading to temporal growth of both waves. Since both waves possess positive group vilocities, i.e., $\left(\mathrm{v}_{\mathrm{gr}}\right)_{\Omega_{0}}\left(\mathrm{v}_{\mathrm{gr}}\right)_{\text {llelix }}>0$, however, ve expect the instability to be convective rather than absolute. Instead of a growth rate, $\Gamma$, the growth is characterized by a gain factor, $\mathrm{K}=\Gamma / \mathrm{v}_{\mathrm{gr}}$. To study the physical evolution of the instability, we have employed two dimensional particle simulations.

The particle simulation code CCUBE is fully electromagnetic and relativistic in cwo orthogonal curvilinear ccordinates and three corresponding velocity coordinates. It has been described elsewhere. ${ }^{13}$ By using a canonical momentum particle algorithm, very relativistic electron beams can be simulated, avoiding virulent numerical Cerenkov instabilities with only nominal smoothing. The geometry flexibility implicit in using general curvilinear coordinates is, furthermore, a great advancage in simulating complex beam configurations.

The beam/helix configuration used in the wave growth simulations is depicted schematically in Fig. 14. The helix was modeiej through the conditions (7a; and (7d), applied at a predetermined radius. (The continuity conditions (7b) and (7c) are satisfied automatically in the sield solving algorithm.) Since practical considerations suggest that the helix should be electrically tied to the outer conducting wall, we precharged the helix so that when the beam was present $\phi=0$ when $r>R_{H}$. Beam particles were injected at $z=$ with momentum and energy adjusted to account for equilibrium rotation and space charge effects. For these cases, there was no transverse thermal spread. The beam could be modulated at injection to launch cyclotron waves of any desired frequency and amplitude. With transmitting boundary conditions on 
fields and particles, this model simulated an infinite beam/helix system, albeit with wave launching occurring only at the $z=0$.

Figure 7 showed the 1 inear instability when $\Omega_{0}=1.65 \omega_{\mathrm{p}}, \gamma_{0}=7.0$, $v=1.75, \mathrm{P}_{\mathrm{H}}=3.2 \mathrm{c} / \mathrm{c}_{\mathrm{p}}, \mathrm{K}_{\mathrm{H}}=4.8 \mathrm{c} / \mathrm{\omega j}_{\mathrm{p}}$ and $\psi=15^{\circ}$. Feak temporal growth $\Gamma_{\max }=0.022 \omega_{p}$, was predicted at $k=0.35 \omega_{p} / c$ and $\omega=0.104 \omega_{p}$. When these parameters were simulated in a system of length $L=100 \mathrm{c} / \mathrm{w}_{\mathrm{p}}$, with a launched wave at an amplitude of $11 \mathrm{kV}$, the wave exhibited the expected spatial amplification. Figure 15 shows phase plots of $\omega_{p} r / c, \gamma \beta_{r}, r y \beta_{\theta}$, and $\gamma_{0}-1$ versus $\omega_{p} z / c$. In addition, probes placed at $z=12.5,37.6$, 63.7, and $88.9 \mathrm{c} / \omega_{D}$ were used to analyze the spatial behavior in more detail. The $E_{\theta}$ probe at the helix radius was especially useful as there were no equilibrium $E_{\theta}$ fields to mask the signal. Figure 16 shows a typical $E_{\theta}$ time history at $z=88.9 \mathrm{c} / \omega_{p}, r=3.2 \mathrm{c} / \omega_{p}$. The magnitude of the amplified wave shown in Fig. 16 is constant ro within $9 \%$ over nearly $\omega_{p} t=400$.

The magnitude of the wave is roughly 40 times that of the launched signal. Figure 16 shows the spectral analysis of the probe signal. The large amplitude wave has a frequency of $w=.11 \omega_{\mathrm{p}}$. Finally, Fig. 17 shows the magnitude of the unstable wave at the four probe positions. Although there vas a 15\% uncertainty in determining the steady signal level from the plots, an amplification rate of $K=0.041 \pm 0.003 \omega_{p} / c$ was inferred. Since the group velocity of the unstable mode was $v_{\mathrm{gr}} \cong 0.6 \mathrm{c}$, this implied a corresponding growth rate of $\Gamma=0.025 \omega_{p}$. The agreement with theory was felt to be excellent.

The theoretical growth curve is fairly flat near peak growch. To check this we performed two additional simulations with the same parameters as above, except the launched waves were at $r=0.31 \omega_{p} / c$ and $k=0.40 \omega_{p} / c$. These had theoretical growth rates of $\Gamma=0.0156 \omega_{p}$ and $\Gamma=0.0161 \omega_{p}$, 
respectively. Growth at the expected rates was again observed, although some broadband amplification was also seen. No nonlinear saturation mechanism has been observed in any of these simulations.

The above simulations, and others, did not show strong saturation of the growth, but merely convective limitation due to the finite amplification length. Nevertheless, growth in cyclotron wave amplitude is accompanied by increasing modulation of the beam radjus, as Fig. 17 shows. Since there was only a limited beam/helix separation, the beam edge must ultimately intersect the helix. Beam contact would certainly destroy the anisotropic conduction properijes of the helix. This effect would not be seen in the simulations. The presence of charge and current densities exterior to the helix, however, do strongly distort the field distributions. Any resemblance between this configuration and the one analyzed in the earlier section would be extremely unlikely, so we conclude that stable amplification would cease at that point. Ultimately though, the effect of unchecked wave amplification is best studied by simulating an amplifier with a longer growth region.

A simulation was performed with similar parameters to the one shown in Fig. 15 except $R_{H}=3.8, \Omega_{0}=2.0 \omega_{p}$, and $\mathrm{L}=160 \mathrm{c} / \omega_{\mathrm{p}}$. Wave growth was unabated until the beam hit the wall. Since the cyclotron wave potential is related to the fractional beam modulation ${ }^{13}$

$$
\frac{\Delta \phi}{\gamma_{0}}=\frac{v}{\gamma_{0}} \frac{\Delta\left(R_{B}^{2}\right)}{R_{B}^{2}}\left(1+\frac{1}{4} k^{2} R_{B}^{2}\right)^{-1} \text {, }
$$

the simulation beam/helix separation of $1.15 \mathrm{c} / \omega_{\mathrm{p}}$ corresponded to 
a maximum wave potential of $0.9 \mathrm{MeV}$. Inclusion of the equillbrium space charge potential, however, shows that the beam energy is not large enough to sustain such fields. With $\gamma_{0}=7$, the maximum observed potential was $1.9 \mathrm{MeV}$, but the bean disruption was so great that all coherence was rapidly lost downstream. The naximum wave potential on the order of $1 \mathrm{MeV}$ gave fields of $0.3-0.5 \mathrm{MeV} / \mathrm{cm}$. Other simulations with greater beam/helix separations showed slightly larger potentials. Increasing the helix radius, however, also decreases the space charge limiting current,

$$
I_{L}=\left(\gamma_{0}^{2 / 3}-1\right)^{3 / 2}\left(\frac{m c^{3}}{e}\right)\left[1+2 \ln \left(R_{H} / R_{B}\right)\right]^{-1}
$$

At the minimum of the radial modulatica, the limiting current can be locally exceeded by the wave, before it hits the helix. Thus when the total potential exceeds a certain 1imit, $-\mathrm{e}\left(\phi_{\mathrm{c}}+\phi_{\text {wave }}\right)>\mathrm{nc}^{2}\left(\gamma_{0}-\gamma_{0}^{1 / 3}\right)$, beam propagation will be inhibited. Although this is somewhat higher than the peak observed potentials, it could conceivably be responsible for the virtual cathode formation observed in simulations with the larger separations. While the instability, thus, did not saturate at a low level, its nonlinear state after hitting the helix was not suitable for collective ion acceleration purposes either. Successful demonstration of wave growth without losing coherence requires simulations with realistic waveguide terminations. 
V. CYCLOTRON WAVE CROWTH IN A EINITE WAVEGUIDE

In the previous section, we ignored the fact that in any real system, the wave growth section will. be finite in length and moreover, will be mechanically coupled to successive components of an experimental device. The finite length and terminations of the wave growth section can significantly effect both the growth of the cyclotr $n$ wave and its extraction from the wave growth section.

The unstable cyclotron wave in the helical guide possesses; a different radial eigenmode structure than does the mode in the presence of a single drift tube. It is the latter in winch it is hoped to be able to accelerate ions. Closely related to this problem is that of decoupling the electromagnetic components of the normal mode of the beam helix system from the beam cyclotron mode, which is principally clectrostatic, at the point of extraction. Failure to do this could lead to reflections and possibly potentially dangerous feedback. These questions relating to the extraction problem have not been addressed here and are the subject of ongoing research.

Finally, the equilibrium on which the cyclotron waves are grown is Influenced by end effects. In the simulations previously discussed, the helix was artificially precharged to model a system of infinite length. However, in a finite system, with the helix grounded to the outer conduction wall, the beam will induce a transient charging wave on the helix. This wave is first launched off the end of the system through which the beam is introduced, and later from the exit end of the system when the head of the beam leaves the wave growth section. In a lossless system, these transients will persist and can lead to several difficulties when trying to grow the 
cyclotron wave. It is this problem of the self-consistent charging of the finite beam-helix systern that we consider here.

To understand the charging problem better, it is helpful to consider the schematic in F1g. 18. With no beam present, the outer conductor, two flanges at the ends and the helix are all at the same potential. The flanges and outer wall always remain at this potential, but, once the beam propagates beyond the near flange, the space charge in the beam establishes a different potential at the helix. In order to bring the potential at the helix back to that of the outer conductor, currents flow in the helix. of course, this charging wave must be a stiperposition of normal modes of the helical waveguide. A similar wave travelling back up the guide is induced when the head of the beam reaches the far flange, since the beam head moves faster than the charging wave. These currents will continue to be reflected from the ends of the wave growth section unless some mechanism is employed to damp them. Since these currents can generate a time dependent $B_{z}$ field which is an appreciable fraction of the guide field, it is important to eliminate them.

One can examine the problem from the point of view of transmission line theory. We calculate an inductance/unit length and capacitance/unit length simply by computing the number of flux linkages/total current and the total charge/voltage difference between conductors. ${ }^{14}$ The expressions for the inductance/unit length and capacitance/unit length are then

$$
\begin{aligned}
& \mathcal{L}=\frac{1}{c}\left[\cot ^{2}\left(1-\frac{R_{H}^{2}}{R_{W}^{2}}\right)+\ln \left(\frac{R_{W}^{2}}{R_{H}^{2}}\right)\right] \\
& c=\frac{1}{c} \ln \left(\frac{R_{W}^{2}}{R_{H}^{2}}\right) .
\end{aligned}
$$


From transmission 1 ine theory one then defines a characteristic impedance wy $z_{0}=(\mathcal{L} / \mathcal{C})^{1 / 2}$. We can further define the phase velocity $v_{p h}$ by $v_{\mathrm{ph}} / \mathrm{c}=(\mathcal{L C})^{-1 / 2}$. Using our expressions Eqs. (25a) and (25b) above, we have

$$
z_{0}=\left[\frac{\cot ^{2} \psi\left(1-R_{H}^{2} / R_{W}^{2}\right)}{\ln \left(R_{W}^{2} / R_{H}^{2}\right)}+1\right]^{1 / 2}
$$

and

$$
v_{p h} / c=\left[\frac{\ln \left(R_{W}^{2} / R_{H}^{2}\right) \tan ^{2} \psi}{\left(1-R_{H}^{2} / R_{W}^{2}\right)+2 n\left(R_{W}^{2} / R_{H}^{2}\right) \tan ^{2} \psi}\right]
$$

We can put the transmission line results on firmer ground. If one solves Maxwell's equations in the coaxial guide with helical center conductor that forms the wave growth section, the following dispersion relation is obtained :

$$
\frac{-q^{2}}{k^{2} \cot ^{2} \psi}\left(1+\frac{k_{z} n}{q^{2} a} \cot \theta\right)^{2}=\frac{I_{n}^{\prime}\left(q R_{H}\right) K_{n}^{\prime}\left(q R_{H}\right)}{I_{n}\left(q R_{H}\right) K_{n}\left(q R_{H}\right)}\left[\begin{array}{c}
1-\frac{K_{n}^{\prime}\left(q R_{W}\right) I_{n}^{\prime}\left(q R_{H}\right)}{I_{n}^{\prime}\left(q R_{W}\right) K_{n}^{\prime}\left(q R_{H}\right)} \\
1-\frac{K_{n}\left(q R_{W}\right) I_{n}\left(q R_{H}\right)}{I_{n}\left(q R_{W}\right) K_{n}\left(q R_{H}\right)}
\end{array}\right],
$$

where $q$ is the perpendicular wavenumber, $k$ the free space wavenumber $\left(\mathrm{k}^{2}=\omega^{2} / \mathrm{c}^{2}\right)$. $I_{\mathrm{n}}$ and $\mathrm{K}_{\mathrm{n}}$ are the modified Bessel functions of order $\mathrm{n}$ and primes denote differentiation with respect to argument. In addition, $q^{2}=k_{z}^{2}-k^{2}$ or $\left(c / v_{p h}\right)^{2}=1+q^{2} / k^{2}$. Note that for the axisymmetric modes in which we are interested, $n=0$. In the iong wavelength limit, for $n=0$, the dispersion relation above becomes

$$
q^{2}=k^{2} \cot ^{2} \psi\left(\frac{1-R_{H}^{2} / R_{W}^{2}}{\ln \left(R_{W}^{2} / R_{H}^{2}\right)}\right)
$$


and thus

$$
c^{2} / v_{p h}^{2}=1+\frac{1-R_{H}^{2} / R_{W}^{2}+\ln \left(R_{W}^{2} / R_{H}^{2}\right) \cot ^{2} \psi}{\ln \left(R_{W}^{2} / R_{H}^{2}\right)},
$$

which is precisely Eq. (26b). Thus we see that the transmission line result is, in fact, the long wavelength (smail $k R_{W}, q R_{W}, g_{W} / R_{H}$ finite) limit. We might expect then, if we terminate the wavegrowth section, at both ends in the characteristic impedance, $Z_{0}$, the long wavelength components in the transients would be absorbed at the ends of the guide.

We have run simulations to test this termination. A schematic is, shown in Fig. 18. The system length is $100 \mathrm{c} / \omega_{\mathrm{p}}$ and $\psi=15^{\circ}$. The guide field is such that $\Omega_{\mathrm{O}}=2 \omega_{\mathrm{p}}$. The guide has $R_{\mathrm{W}}=5.7 \mathrm{c} / \omega_{\mathrm{p}}$ and $R_{\mathrm{W}} / R_{\mathrm{H}}=1.5$. The end terminations were simulated by including a resistive region between the ends of the helix and the flanges as indicated in Fig. 18. The con. ductivity is determined by requiring that a cylindrical annulus one radial cell thick and $\mathrm{N}$ cells long have a value of resistance equal to $\mathrm{Z}_{0} \cdot$ We have run simulations with $\mathrm{N}$ equal to both twenty-five and five. The latter gave slightly better results for reasons which are not clear to us; but, in any case, the difference is negligible in a practical sense.

The beam had an energy of $\gamma_{0}=7$ and radius of $2.65 \mathrm{c} / \mathrm{\omega}_{\mathrm{p}}$. For the bulk of the runs, a so-called "slug", where the bean electrons do not respond to the fields, was used. By introducing a finite risetime on the beam, the strength of the short wavelength components in the transients are reduced relative to the long wavalength modes. We have used a gaussian current rise $I \propto \exp \left(-t^{2} / 2 \tau^{2}\right)$. Even without resistive terminations, the dispersion in the helical guide can smooth the charge transients on the 
helix after a sufficiently long time. With a risetime of $\omega_{p} \tau=500$, $R_{H}=3.2 \mathrm{c} / \omega_{p}$ and $R_{W} / R_{H}=1.5$, the relative charge inhomogeneity is less than $2.5 \%$ at $\omega_{p} t=2000$. In terms of a realistic charge density, this corresponds to a time of $35-70 \mathrm{nsec}$. This is a little longer than one would like but might be acceptable. A finite current will persist without dissipation, however, and this will lead to undesirable kinks in the $B_{z}$ guide field which can excite unwanted beam modes, in particular, the zero frequency cyclotron mode. This will be discussed in more detail shortly. Fig. 19 shows the charge density, $\theta$-component of the current density, and z-component of the magnetic field and current density for such a case, at $\omega_{p} t=2000$, With the helix terminated, the situation can be improved significantly, Slug simulations with a beam risetime of $\omega_{p} \tau=25$, are suimarized in four illustrations. The parameters are as indicated above.

Fig. 20 displays the $z$ and $\theta$ components of the current densicy, $z$ component of the magnetic field and charge density as a function of $z$ at $\omega_{p} t=450$ for an unterminated guide. $J_{z}, J_{\theta}$ and $\rho$ are plotted, parametrically, for several radii, particularly those of the beam and helix. Between the flanges, $J_{\theta}$ flows only on the helix. The charging current transients and severe distrotion in $\mathrm{B}_{z}$ are clear. Figure 21 shows the same quantities as Fig. 20, at the same time, in a guide terminated in the characteristic Impedance $Z_{0}$. The result is dranatically different. Indeed, with a risetime as short as $\omega_{p} \tau=25$, the results are better than we should have expected, given the nature of the approximation $\left(k R_{W} \approx k R_{H} \ll 1\right)$ used to arrive at the result. A numerical study of the characteristic impedance derived by a field theoretic approach in the coaxial guide with helical center conductor $^{13}$ shows that, as $\mathrm{R}_{\mathrm{W}} / \mathrm{R}_{\mathrm{H}} \rightarrow 1$, the characteristic impedance approaches 
Its long wavelength value even for $q R_{H}\left(\approx q R_{W}\right)$ of order unity. Thus, in our system with $R_{W} / R_{H}=1.5$, even waves of moderate frequency are damped by the terminating resistors.

We have also tested a method of termination which employs an absorber made by filling part of the region between the helix and the outer wall with a resistive medium. In particular, we increased the conductivity from zero linearly to a value $\sigma$, over a length in $z$ of $12 \mathrm{c} / \omega_{p}$ and then held the conductivity constant over an additional distance in $\mathrm{z}$ of $12 \mathrm{c} / \mathrm{\omega}_{\mathrm{p}}$. This absorber ended at either the far flange or the far termination resistor if one was used. The simulations to be described had $\sigma_{0}=0.15 \omega_{p}$ and is such that $\sigma_{0}<\omega$ for the dominant high frequency guide mode which has $\omega \simeq 0.6 \omega_{p}$. Furthermore we choose the gradient length, $\ell>2 \pi / k$ where $k$ is the free space wave number. The condition on $\sigma_{0}$ implies that $2 \pi / k>\delta$ where $\delta$ is the skin depth.

Figure 22, which is a simulation with only the absorber shows that the absorber does help reduce the transients but is not nearly as effective in reducing them as is termination in $\mathrm{Z}_{\mathrm{o}}$. Furthermore, in a lossless helix, a $\mathrm{J}_{\theta}$ will persist with only an absorber, and thus the kinks in $\mathrm{B}_{2}$ will remain. Figure 23 is a simulation with absorber included in a terminated guide, and by comparing this figure with Fig. 21, we see that the absorbing material is effective in reducing the long-1ived but relatively small high-frequency components for wich the expression for the characteristic impedance is no longer valld. Such components are probably not serious for the wave growth experiments, but in any case, we do know how to get a handle on them. We remark that we have made 1ittle effort to optimize the absorber at this point and thus one could possibly improve upon its performance with additional study both theoretically and by simulation should the need arise. 
Two particle simulations to test these results with a beam which could respond to the helix fields were carried out. The parameters for the first were $\gamma_{0}=7, \Omega_{0}=2.0 \omega_{p}, R_{B}=2.65 \mathrm{c} / \omega_{p}, R_{W}=5.7 \mathrm{c} / \omega_{p}, R_{H}=3.8 \mathrm{c} / \omega_{p}$, and $L=160 \mathrm{c} / \omega_{p}$. An absorber was included and extended from $z=135 \mathrm{c} / \omega_{p}$ to the right flange. There was no termination and thus a current would persist on the helix. The beam had a risetime of $\omega_{p} \tau=300$. At $\omega_{p} t=1000$, a test wave was Iaunched. By this time, steady beam conditions had been established.

As noted above, the $\mathrm{B}_{2}$ field kink near the helix-flange interface did excite the zero frequency cyclotron wave. Theory gives for the wavelength $\lambda_{z f}$ of this mode a value $\lambda_{z f}=2 \pi v_{0} \gamma_{0} / \Omega_{0}$ which in our case is $\lambda_{z f}=22 \mathrm{c} / \omega_{0}$. The observed wavelength was $\lambda=21.5 \mathrm{c} / \omega_{\mathrm{p}}$. At $\omega_{\mathrm{p}} \mathrm{t}=1000$ a test signal with $\omega=0.131 \omega_{p}, k=0.49 \mathrm{c} / \omega_{p}$ and amplitude $22 \mathrm{keV}$ was introduced. Cyclotron wave growth occurred thereafter, much as in the precharged, open-ended simulations. The fact that the instability persisted in the presence of the zero-frequency wave was very encouraging, though beating of the two finite amplitude waves may have contributed to the eventual beam disruption. Since the more quiescent configuration in which terminations damp the transients and "DC" helix current was more suitable, we did not pursue this wave growth system further.

The second simulation was performed with virtually the same parameters as the first. The exceptions were the inclusion of terminations, removal of the absorber, and a shortening of the risetime to $\omega_{p} \tau=200$. Recall that the shortening of the risetime is a more stringent test of the system. While we had demonstrated the efficiency of the terminations in slug simulations with risetimes as short as $\omega_{p} t=25$, prelimina:y full particle simulations indicated that a somewhat longer risetime might be needed as a sharp 
front edge on the bean proved to be an uncomfortably efficient exciter of slow cyclotron waves. These waves were created at a high enough level that only a small amount of growth was required to bring them to the helix. The ensuing beam disruption was eventually carried out of the system by the helix charging pulse. Residual turbulence, however, prevented the beam froil ever calming down to the point that stable amplification was possible.

With a risetime of $\omega_{p} \tau=200$, the matched terminations performed as anticipated. Initial current transients on the helix led to field kinks and the zero-frequency mode. As the current decayed, the fields straightened and without an exciter, the zero-frequency mode was convected cut of the system. At $\omega_{p} t=900$, the test wave was launched after which successful growth was observed. After approximately $\omega_{p} t=1250$, the waves were of sufficient amplitude to cause the beam to strike the helix, but the waves persisted to $w_{p} t=1450$, which was the end of the run. Figure $24 a$ and $b$ shows two frames from the unterminated and terminated simulations, respectively. The frame from the unterminated run is at $\omega_{p} t=1248$ and for the terminated run $\omega_{\mathrm{p}} \mathrm{t}=1155$. This gives the same time interval from initiation of the tickler in both cases. Even with the longer risetime, the zero-frequency mode (longer of the two modulatıon wavelengths) is clearly present f.n the unterminated case, and some beating of this mode with the slow cyclotron mode is evident. In clear contrast, the terminated run is free of the zero-frequency mode even with its shorter rsetime. Recall, too, that this frame is $93 \omega_{p} t$ earlier than the one from the unterminated simulation. We are confident that this aspect of the finite helical wave growth section has been resolved. We are now addressing the problems involved with the extraction of the wave from the helix amplifier. 
VI. CONCLUSIONS AND DISCUSSION

The results of this study can now be summarized. We reviewed the linear theory of cyclotron beam modes, helical waveguide modes, and the coupling between them. The results of this analysis, although potentiaily useful, were of uncertain value, since we could not estimate the errors induced through our assumed, simple equilibrinm. Neglect of radial variations in beam energy and rotation, in particular, were suspect. To remedy this oversight, we obtained exact numerical dispersion relations in realistic, inhomogeneous beam profiles. These demonstrated regions of validity for che analytic expressions, as well as behavior of the stable and unstable waves outside the analytic regimes. In particular, long wavelength waves, $k c / \omega_{p} \ll I$, were well described by the analytic theory, as was their unstable coupling. The numerical study, however, also exhibited a region in which inhomogeneity of the cyclotron resonance led to disruption of stable eigenmodes. Perturbations within this region of $\omega-k$ space would be susceptible to secular decay, due to phase mixing. This was not an immediate concern in the present study, for tiie stable cyclotron modes were found to exist just outside the branch cut region. Although the instability could shift the mode toward that region, there was little evidence of the highly spiked field signature in the unstable waves, so long as $\mathrm{kc} / \mathrm{\omega}_{\mathrm{p}} \tilde{<} 2$. The primary unanswered question concerns the behavior of the large amplitude cyclotron wave after it physically leaves the unstable amplifying section. This is being studied at present.

The analytic and numerical studies indicated the viability of cyclotron wave growth, via coupling with a helical waveguide mode. These studies 
were all within the context of linear theory, however, and moreover did not consider the convective character of the process. The nonlinear state of the instability was completely unknown. We addressed these questions with electromagnetic, relativistic particle simulations in cylindrical geometry. Two separate simulation configurations were employed. Int the first, open-ended boundary conditions modeled the propagation of an electron beam in an infinite helical waveguide. Since the simulation length was finite, large waves could leave after only a finite amplificatIon length, but in all other respects, such as end effects, initial translents, reflections, and so forth, the simulations were open ended. Calculations performed on this configuration werc found to agree with linear theory very adequately. Predicted gain coefficients, shape of the gain curve, and unstable frequencies were within $15 \%$ of the observed simulation values. Furthermore, no nonlinear mechanism was observed to inhibit the wave growth, at least up to wave magnitudes sufficient to strike the helix. In the second configuration, finite terminations were included in the simulations, corresponding to perfectly conducting flanges. Since the helix was electrically tied to the conducting outer wall structure, initial transients associated with establishment of a beam/helix equilibrium could occur. Unless the helix/wall interface was properly matched, these transients were found to be very long-lived, and capable of inducing sufficient axial inhomogeneity to preclude successful wave amplification. Elimination of high frequency components required not only the usual series, low frequency impedance, but shunt volume resistance as well. When these were included, establishment of homogeneous equilibrium in reasonable experimental time swales was found to be feasible. Neglect of these elements, 
how'ever, would almost certainly be disastrous for amplifier performance. Once the self-consistent equilibrium was set up, introduction of a sma11 signal wave resulted in the same, stable amplifier characteristics seen in the open ended simulations.

Overa11, the studies have many ramifications for a physical cyclotron wave amplifier. They have been generally encouraging, but problem areas requiring further study have been identified. On the positive side, we have shown that the helix conditions can be established on acceptable time scales with reasonable beam and helix parameters. The helix induced cyclotron wave amplification was at the expected rate, narrowband, and nonturbu1ent. Wave and beam breakup, on the other hand, were observed if the wave became too large, i.e., its peaks intersected the helix. Although the resulting state was unsatisfactcry as calculated, the fact that accompanying materfals problems were not included leads us to conclude that beam contact with the helix would almost certainly terminate the amplifier. Contact is not inevitable, of course, since the length of the system can be appropriately tailored, but it should be considered because it is a deleterious effect.

In light of the negative aspects of wave/helix contact, it should be noted that nonaxisymmetric cyclotron modes, $m \neq 0$, have been deliberately omitted from this study. These "kink" modes bend the entire beam closer to the helix, thereby agravating the helix contact problem. Our omission of this important area is based on three factors. First, the $m=0$ wave, the one envistoned for collective ion acceleration, had not been studied under realistic conditions. Until the viability of growth of that wave had been 
demonstrated, there seemed little value in considering competition with undesirable modes. Second, Bourianoff, Cornet, et al, had analyzed the linear theory of growth of general nonaxisymmetric, $m \neq 0$, waves in considerable detail. They have also simulated growth of the $m=1$ wave in cartesian geometry, albe:t driven by a periodic loop structure, not a helix. Finally, closely related to the second factor, realistic cylindrical simulations of helix induced growth of the $m=1$ wave are intrinsically three dimensional. Although a three-dimensional linearized simulation code is currently being developed by us, the prospect for fully nonlinear three-dimensional simulations in the near future is dim, computer hardware being the key limitation.

There are several interesting questions which arise when less than ideal electron beams are employed. Injection of the beam through an anode foil can generate zero-frequency bouncing as well as scatter. We have already seen that the mere presence of bouncing does not significantly degrade the amplifier, providing the wavelength of the bounce is separated from that of the instability. The effect when the wavelengths are nearly coincident, however, is moot. Also, scatter is the same as transverse thermal spread, so Landau damping could conceivably modify the linear and nonlinear state of the beam modes. Except for very low phase velocities, this should not be significant. Finally, long time voltage rise or droop of the beam may shift the beam mode out of resonance. The effect of voltage changes on amplifier performance is difficult to judge, though. Numerical studies of that problem are feasible and will be pursued in the future.

In conclusion, the helical slow wave structure seems to be very satisfactory as a slow cyclotron beam mode amplifier. There are still problem 
areas to be investigated, but we have not encountered any fundamental difficulties yet which would preclude construction of a successful device.

\section{ACKNOWLEDGEMENTS}

We would like to thank Drs. Edward Cornet, George Bourianoff, M. Lee Sloan, Vernon Wong, and W. Richard Shanahan for many stimulating and illuminating discussions on this subject. Research performed under the auspices of the Energy Research and Development Administration. 


\section{BIBLIOGRAPHY}

1. M. L. Sloan and W. E. Drummond, Phys. Rev. Letters 31, 1234 (1973).

2. P. Sprangle, A. T. Drobot, and W. M. Manheimer, Phys. Rev. Letters $\underline{36}$, $1180(1976)$

3. J. H. Malmberg and C. B. Wharton, Phys. Fluids 12, 2600 (1969).

4. K. Mizuno and S. Tanaka, Phys. Rev. Letters 29, 45 (1972).

5. J. H. Wakeren and H. J. Hopman, F'nys. Rev. Letters 28, 295 (1972).

6. K. W. Gentle and J. Lohr, Phys. Fluids 16, 1464 (1973).

7. M. Seid1, W. Carr, D. Boyd, and R. Jones, Phys. Fluids 19, 78 (1976).

8. M. S. Livingston and J. P. Blewett, Particle Accelerators (McGrawHill, New York, 1962).

9. J. R. Pierce, Traveling Wave Tubes (Van Nostrand, New York, 1950).

10. W. E. Drummond, G. I. Bourianoff, E. P. Cornet, D. E. Hasti, W. W. Rienstra, M. L. Sloan, H. V. Wong, J. R. Thompson, and J. R. Uglum, AFWL-TR-75-296 (1976).

11. H. V. Wong (Private Communication, c.f., Appendix J, I-ARA-77-U-47 (ARA-273), June 1977).

12. B. B. Godfrey, Subratted to IEEE Trans. on Plasma Science.

13. B. B. Godfrey, IEEE Trans, on Plasma Science, to be published.

14. W. Sichak, Proc, I.R.E., 42, 1315 (1954). 


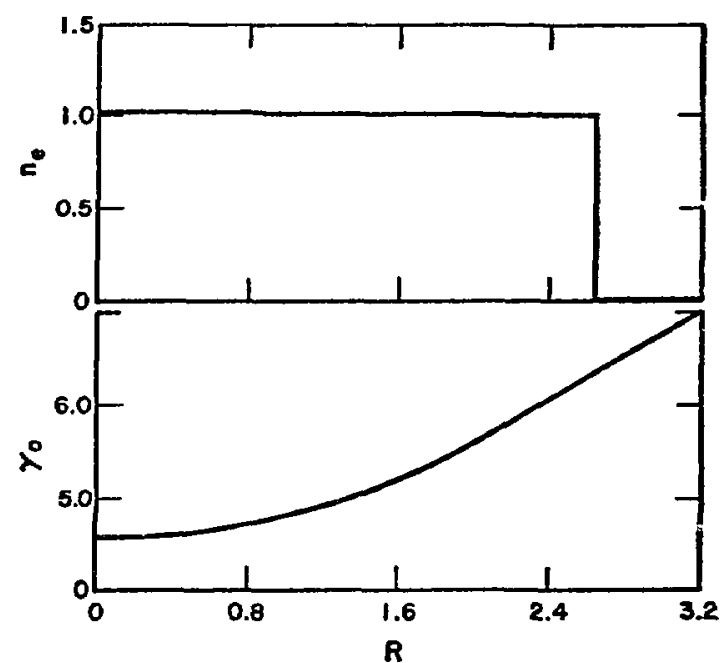

Fig. 1. Equilibrium electron density and energy $\left(\gamma_{0}\right)$ radial profiles, when $\gamma_{0}=7.0$, $\mathrm{R}_{\mathrm{B}}=2.65 \mathrm{c} / \omega_{\mathrm{p}}, \mathrm{R}_{\mathrm{H}}=3.2 \mathrm{c} / \omega_{\mathrm{p}}$, and $\Omega_{\mathrm{o}}=$ $1.65 \omega_{\mathrm{p}}$. The electron density is normalized to the mean beam density, $\vec{n}=\int_{0}^{R_{B}} r n_{e} d r / R_{B} \cdot$ Electron energy, $\gamma_{o}$, has no meaning when $r>R_{B}$, but is depicted to illustrate the equilibrium condition, $\gamma=\gamma_{0}+e \phi / \mathrm{mc}^{2}$. Dimensions are in units of $c / w_{p}$.

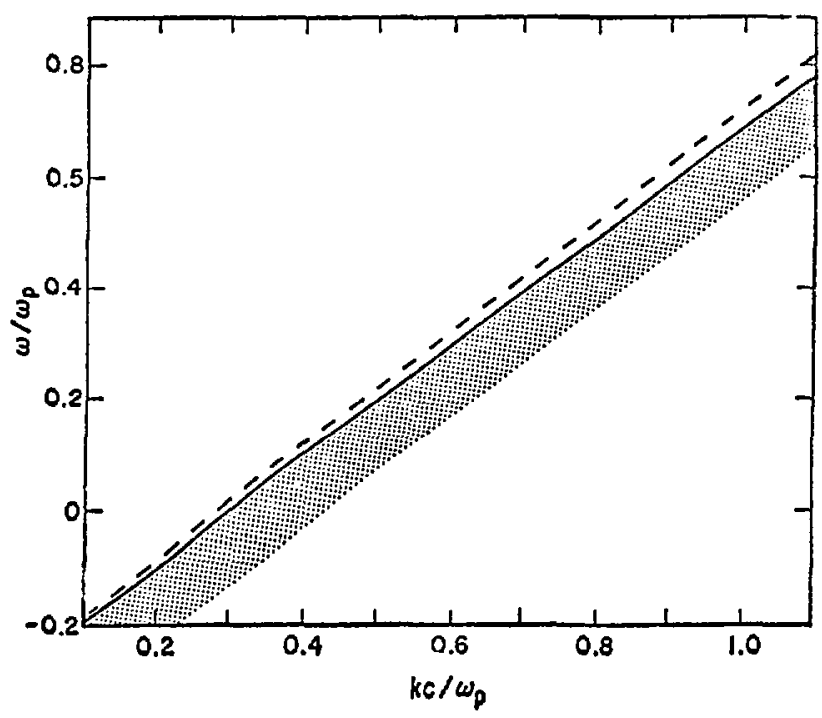

F1g. 4. Slow doppler-shifted cyclotron dispersion relation with some parameters as in Fig. 3, plus $\Omega_{o}=2.0 \omega_{p}$ and $\gamma_{o}=7.0$. Dashed line is approximate expression, $\omega=\mathrm{kv}_{0}-\Omega_{0} / \gamma_{0}$; the shaded region indicates a branch cut region.
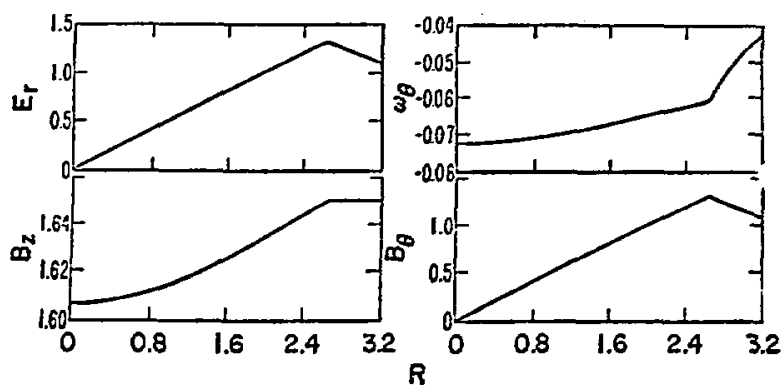

Fig. 2. Equilibrium self-fields for same parameters as in Fig. 1.

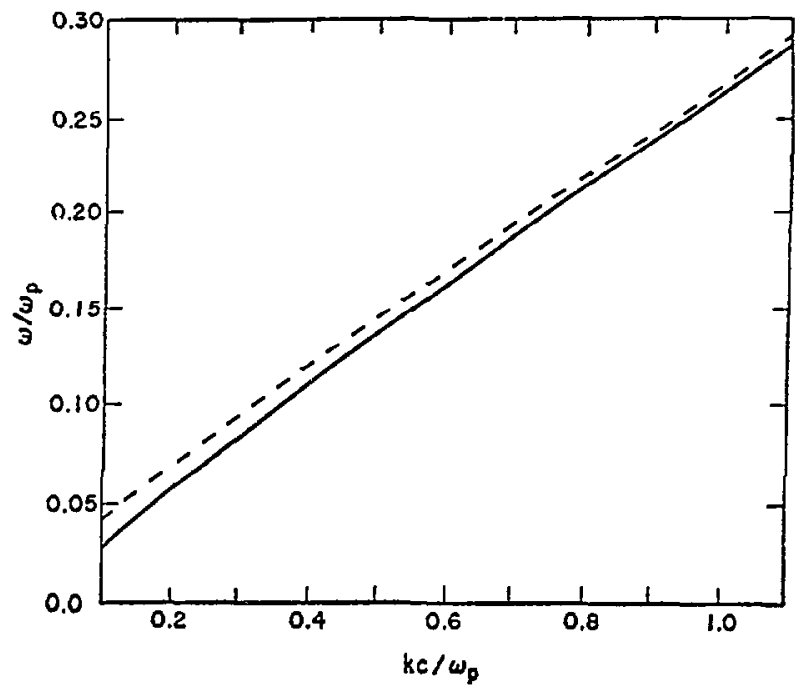

Fig. 3. Electromagnetic helix dispersion relation when $R_{B}=2.65 \mathrm{c} / \omega_{p}, R_{W} / R_{B}=1.5$, and $\psi=15^{8}$. Dashed Iine is Eq. (8). 


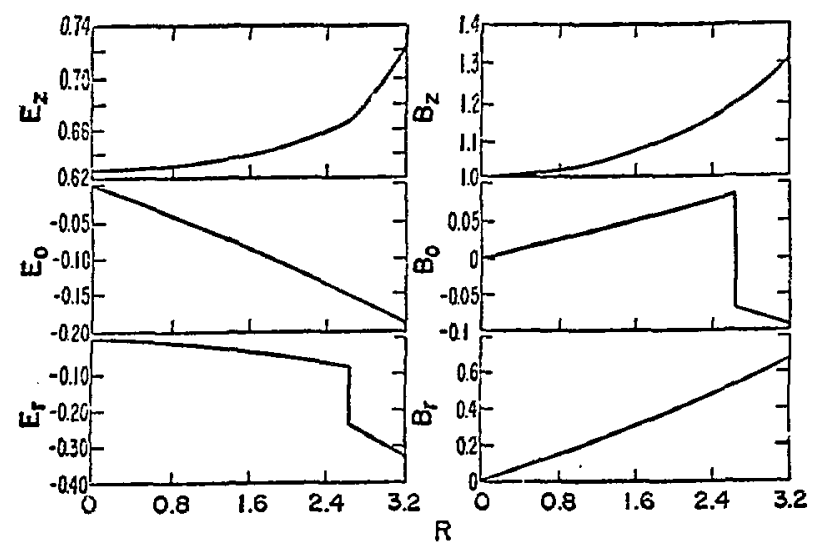

$5(a)$

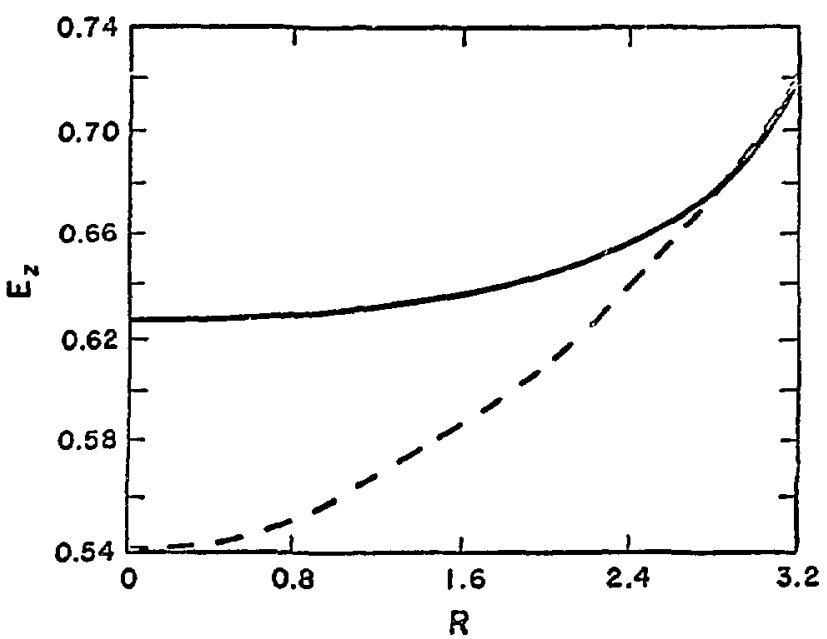

5 (b)

Fig. 5. Radial field structure of electromagnetic helix elgenrade, $R=0.36 \omega_{p} / c, \omega=0.1043 \omega_{p}$, other parameters as in FIg. 3. (a) Field components; (b) Detailed comparison between numerical (solid IIne) and analytic (dashed line) dependence of $\mathrm{E}_{z}$.

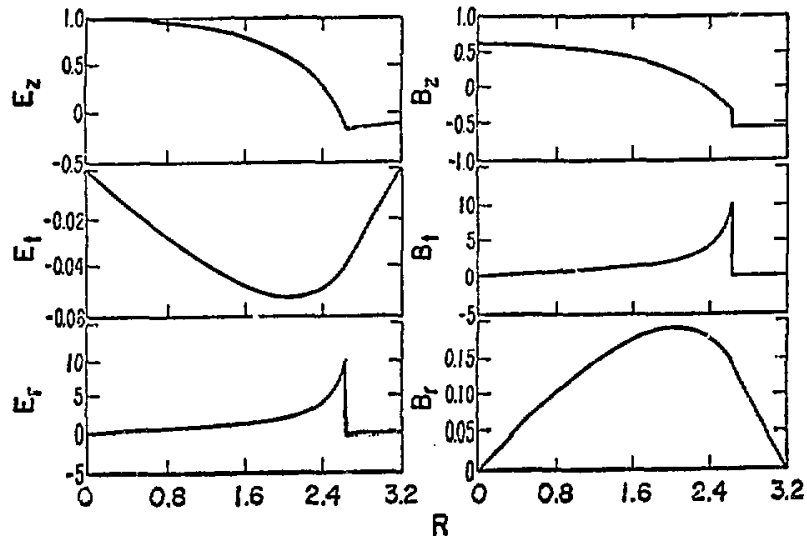

Fig. 6. Radial cyclotror elgenfields, $k=0.42 \omega_{p} / c$,

$\omega=0.116 \omega_{p}$, other parameters a3 $i_{11}$
Fig. 4 .
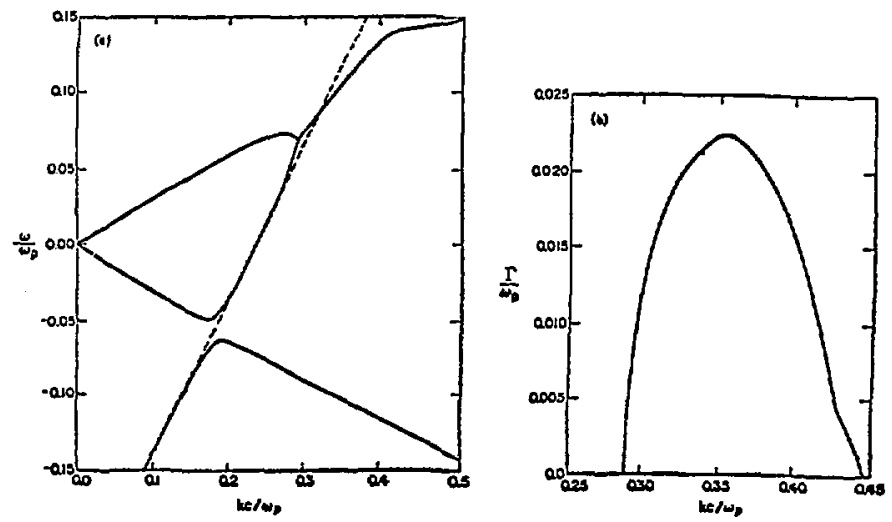

Fig. 7. Dispersion relation showing unstable coupling of a positive energy waveguide mode and a slow cyclotron beail wave, when $\Omega_{\mathrm{o}}=1.65 \omega_{\mathrm{p}}, \gamma_{\mathrm{o}}=7, v=1.75, \psi=15^{\circ}$, other parameters as in Fig. 3.

(a) Frequency as a function of $\mathrm{kc} / \omega_{\mathrm{p}}$. Dashed line indicates the stable cyclotron dispersion. (b) Growth rate as a function of $\mathrm{kc} / \omega_{\mathrm{p}}$. 


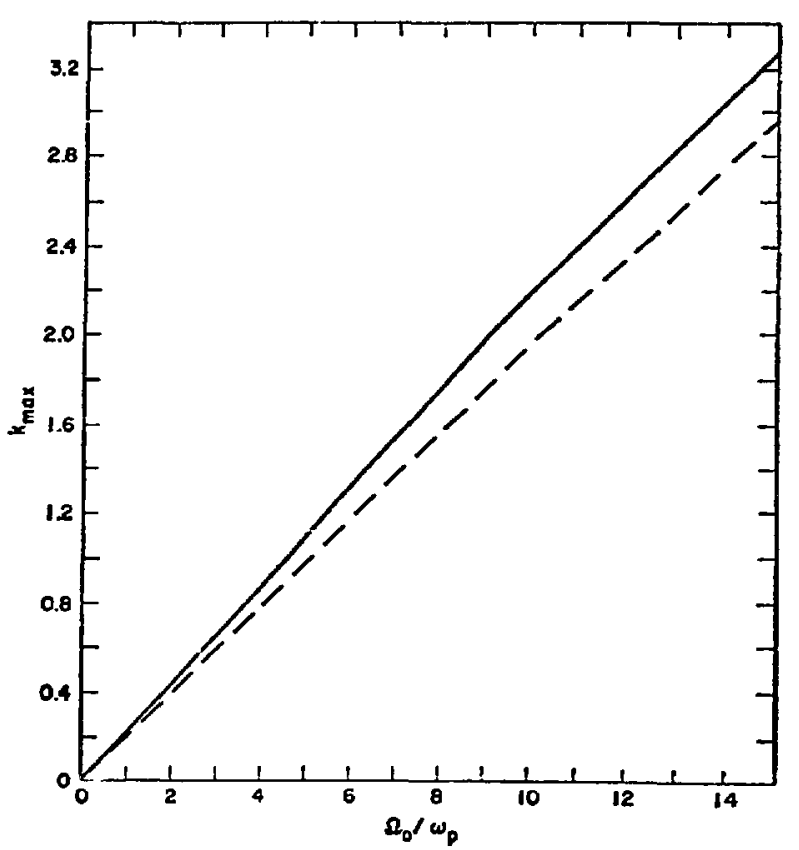

Fig. 8. Wavenumber at which peak growth occurs, $k_{\text {max }}$, as a function of magnetic field, $\Omega_{0} / \omega_{p}$, parameters as in Fig. 4. The dashed line is Fq. (21); the solid, the numerical values.

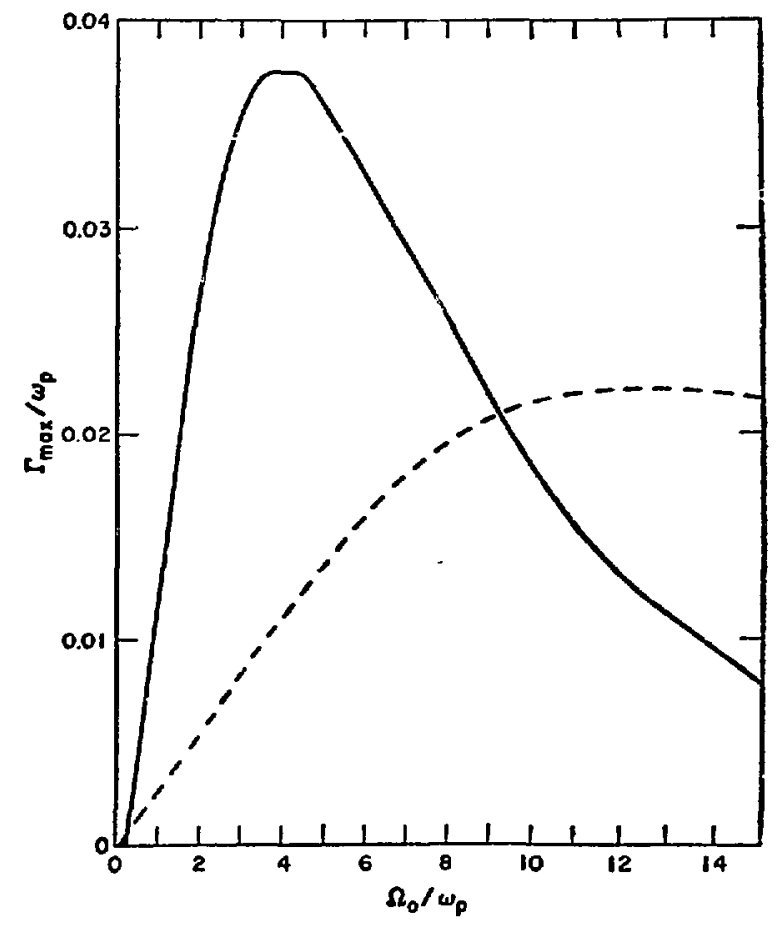

Fig. 9. Peak growth rate, $\Gamma_{\max }$, as a function of normalized magnetic field, same parameters as Fig. 3. Solid line is for $\gamma_{0}=7.0$, dashed for $\gamma_{0}=20$.

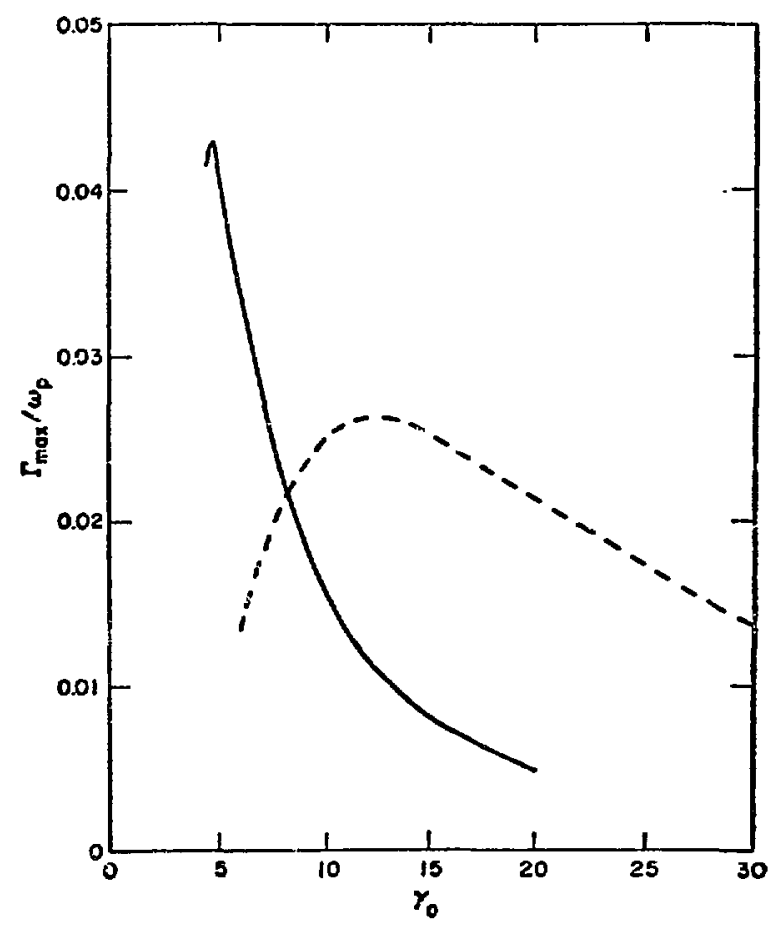

Fig. 10. Peak growth rate as a functiun of $\gamma_{0}$, parameters simflar to Fig. 9. Solid

line is for $\Omega_{0}=2.0 \omega_{\mathrm{p}}$, dashed for

$\Omega_{\mathrm{o}}=10 \mathrm{\omega}_{\mathrm{p}}$. 


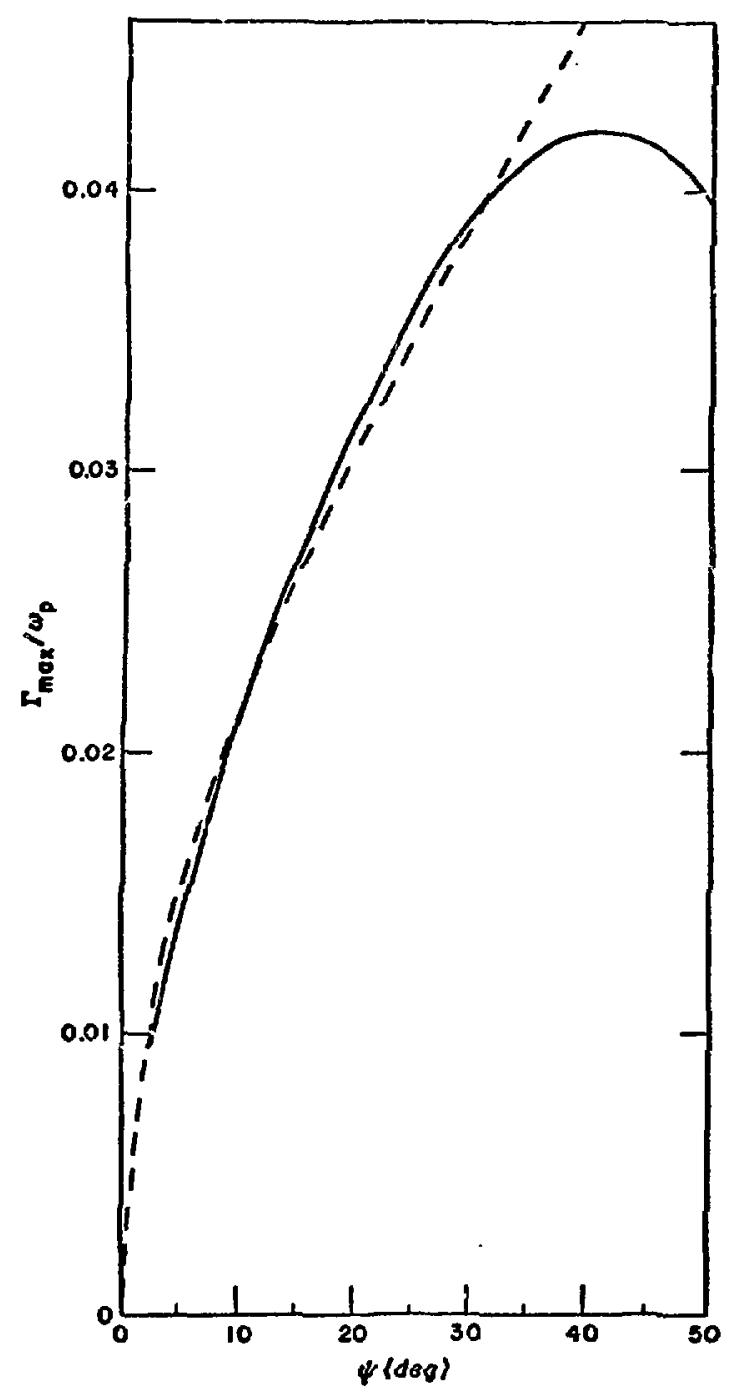

Fig. 11. Peak growth rate as a function of pitch angle $\psi$, same as Fig. $9, \Omega_{0}=2.0 \omega_{p}$, $\gamma_{0}=7.0$. Dashed line shows dependence of $(\tan \psi)^{1 / 2}$ curve.

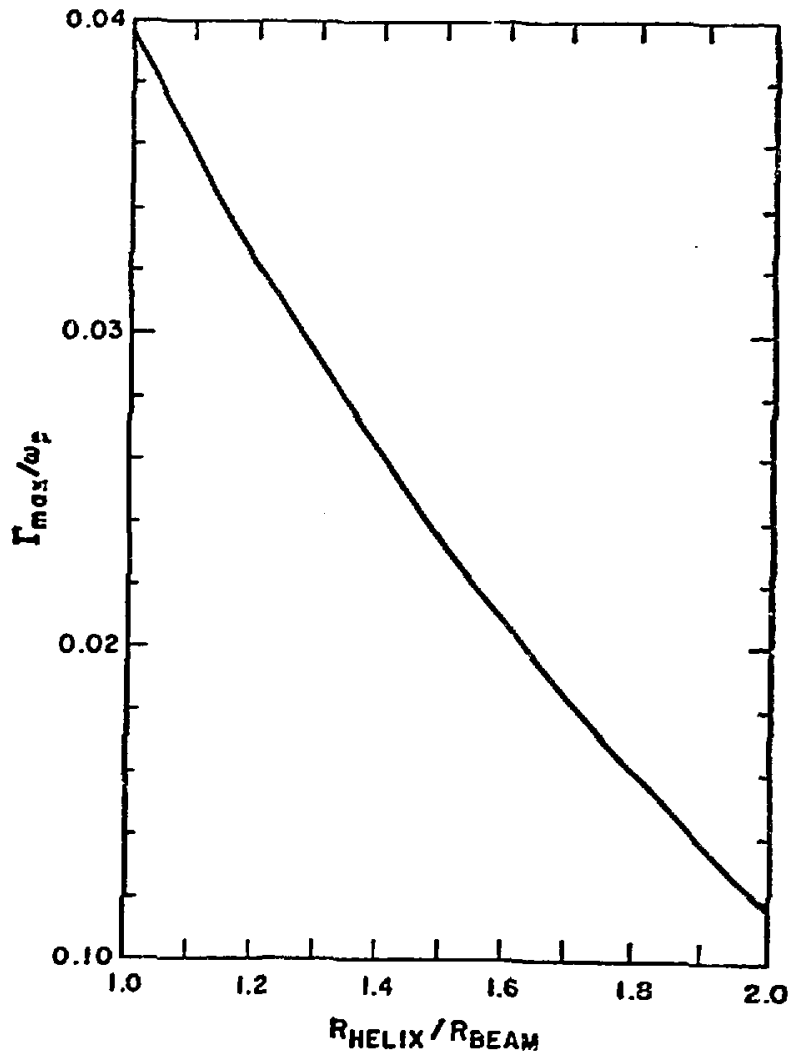

Fig. 12. Dependence of peak growth rate as a function of beam/helix separation, parameters as in Fig. 11, $\psi=15^{\circ}$. 


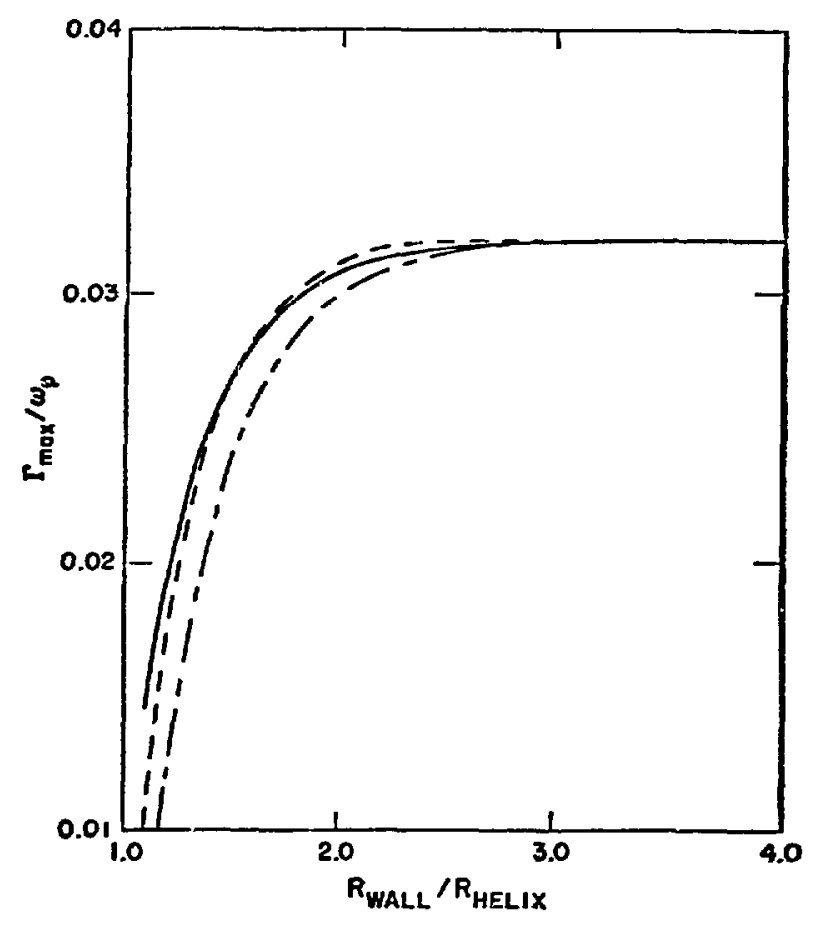

Fig. 13. Dependence of peak growth rate as a function of wall/helix separation, parameters as in Fig. 1?, $\psi=15^{2}$. Numerical result Is the solid line; $(---)$ : $\Gamma_{\max } / \Gamma_{0}=1-\mathrm{a}^{2 \mathrm{q}\left(\mathrm{R}_{\mathrm{H}}-\mathrm{R}_{\mathrm{W}}\right)} ;(---)$ : $I_{\max }^{\max } / \Gamma_{0}^{\circ}=1-k_{o}\left(q R_{W}\right) k_{\perp}\left(q R_{W}\right) / k_{o}\left(q R_{H}\right) k_{\perp}\left(q R_{l}\right)$.

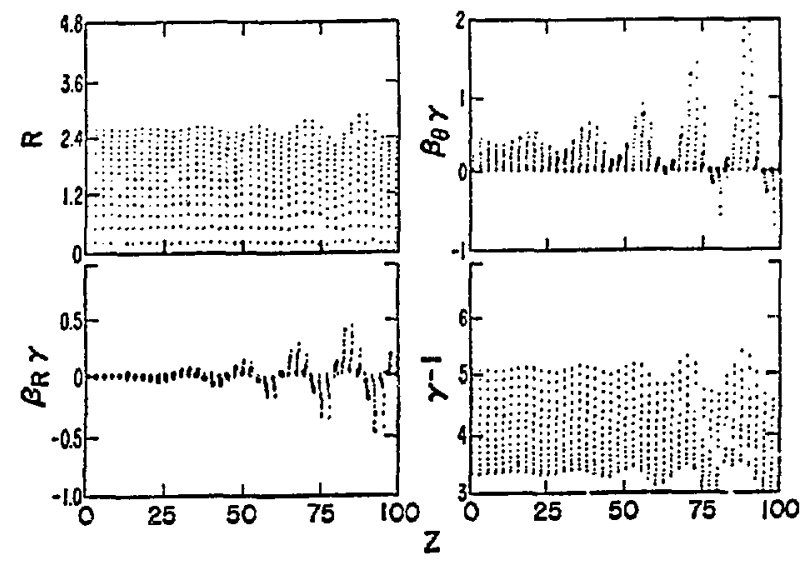

Fig. 15. Phase space plots from a typical simulation showing spatial eyclotron wave amplification, $\Omega_{\mathrm{O}}=1.65 \omega_{\mathrm{p}}, \gamma_{\mathrm{o}}=7.0, \nu=1.75$, $L=100 \mathrm{c} / \omega_{\mathrm{p}}, \mathrm{R}_{\mathrm{H}}=3.2 \mathrm{c} / \omega_{\mathrm{p}}, \mathrm{R}_{\mathrm{W}}=4.8 \mathrm{c} / \mathrm{\omega}_{\mathrm{p}}$ and $\psi=15^{\circ}$. Starting in upper left and going clockwise, the plots show (a) $r-z$ configuration space, (b) angular momentum vs. $\mathrm{z}$, (c.) radial momentum vs. $\mathrm{z}$, and (d) kinetic energy vs. $z$.

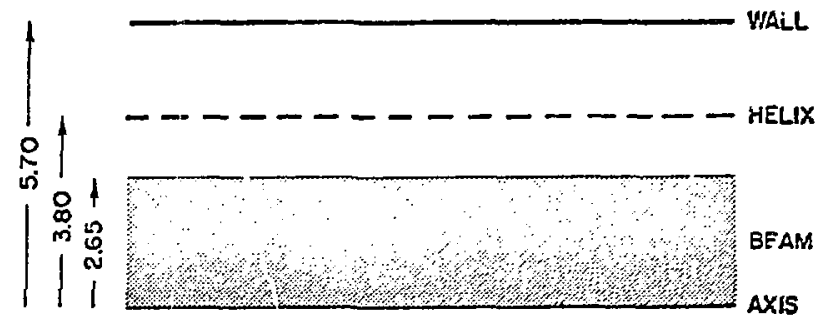

Fig. 14. Schematic of open ended helix simulations. Radial dimensions are in units of $\mathrm{c} / \omega_{\mathrm{p}}$. For a beam density corresponding to $v=1.75, c / \omega_{p}=1.00 \mathrm{~cm}$.
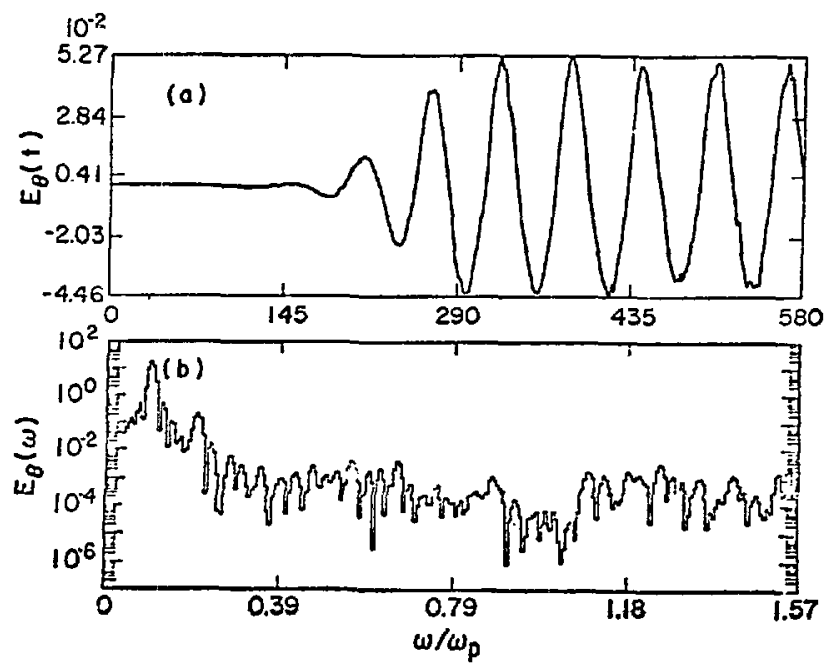

Fig. 16. Time evolution of $E_{\theta}$ field at a fixed position, $r=R_{H}, z=89 \mathrm{c} / \omega_{p}$, same simulation as in Fig. 15. (a) Time history, (b) Fourier analysis of signal, central frequency is $\omega=0.11 \pm 0.01 \omega_{p}$. 


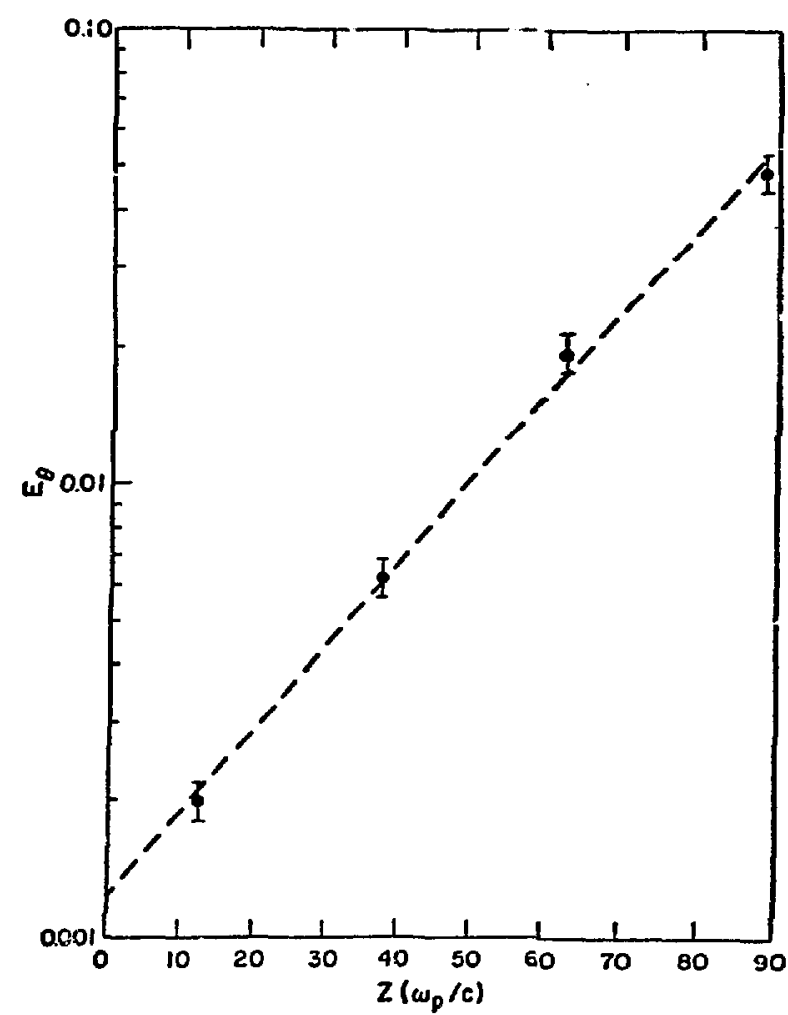

Fig. 17. Mean amplitude of $\mathrm{E}_{\theta}$ fleld as a function of distance, same simulation as in Fig. 15. Dashed line indicates a gain coefflcient of $K=0.041 \pm 0.003 \omega_{p} / c$.
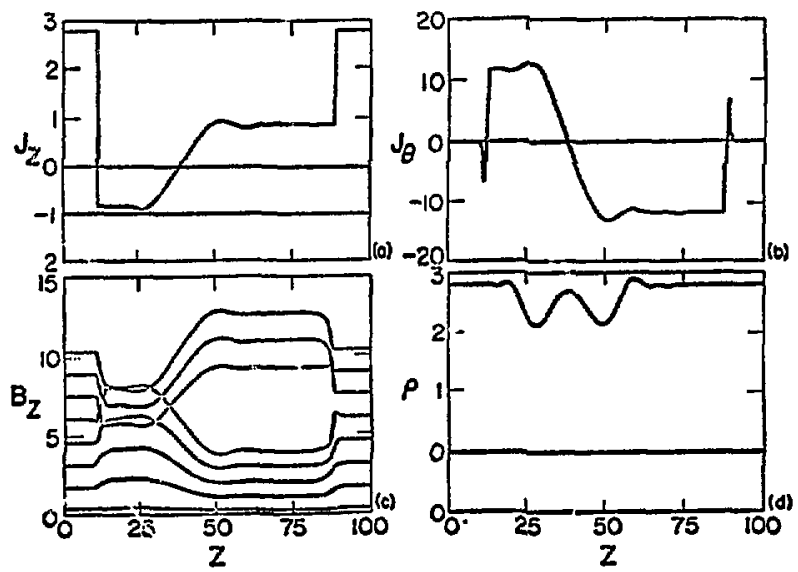

Fig. 20. Uniterminated slug simulation with a risetime of $\tau=25 \omega_{\mathrm{p}}^{-1}$ at $t=450 \omega_{\mathrm{p}}^{-1}$; (a) through (d) as in FIg. 19.

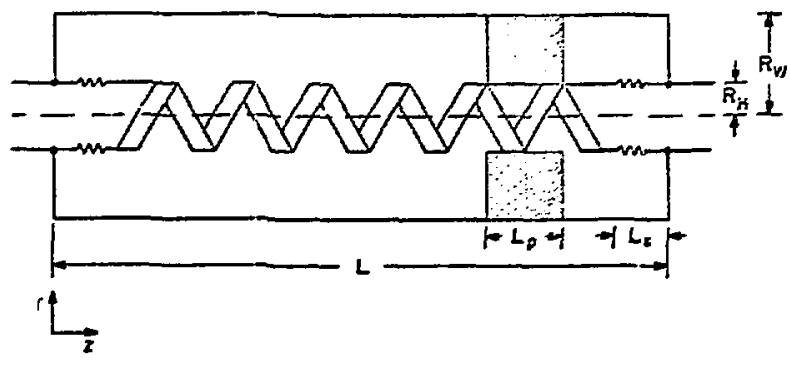

Fig. 18. Schematic of finite, terminated simulations. Shaded areas contain graded shunt rezistance.
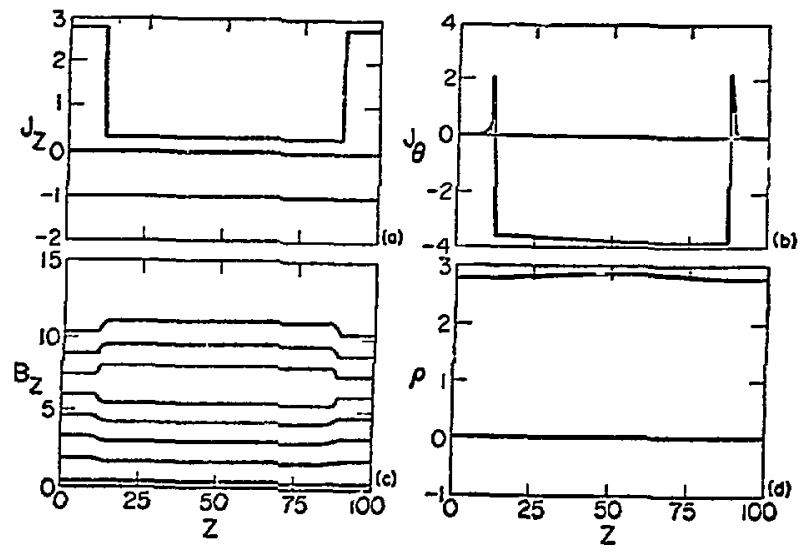

Fig. 19. Unterminated slug simulation with a risetime of $\tau=500 \omega_{\mathrm{p}}^{-1}$ at $t=2000 \omega_{\mathrm{p}}^{-1}$;

(a) $z$-component current density, (b) $\theta-$ component current density, (c) 2-component magnetic fleld, (d) charge density, all versus axial position.
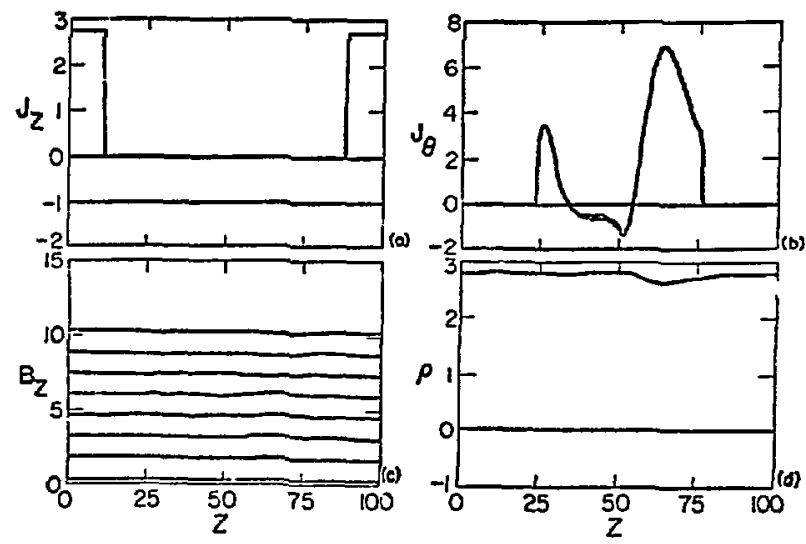

Fig. 21. Slug simulation terminated by $z_{0}$ at $t=450 \omega_{p}^{-1}$ and $\tau=25 \omega_{p}^{-1}$, (a) through (d) as in Fig. 19. 

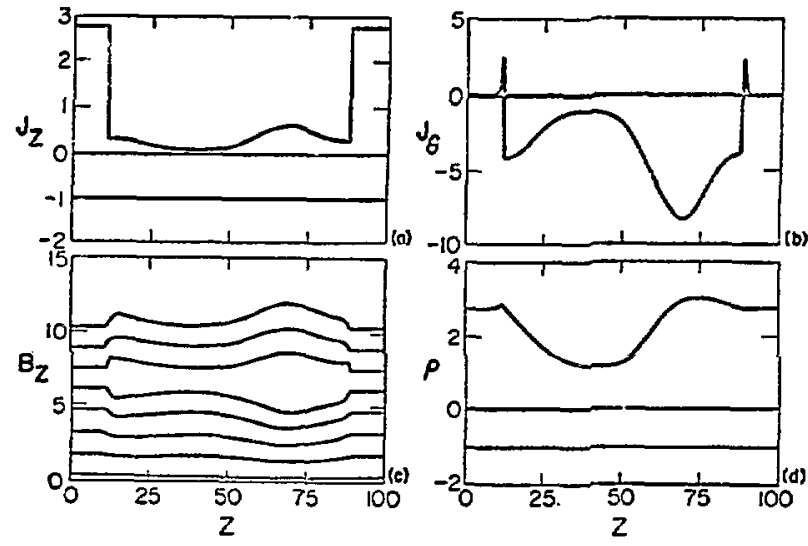

Fig. 22. Slug simulation with an absorbing material between the helix and outer wall, $t=450 \omega_{\mathrm{p}}^{-1}$ and $\tau=25 \omega_{\mathrm{p}}^{-1}$, (a) through
(d) as $1 \mathrm{n}$ Fig. 19 .
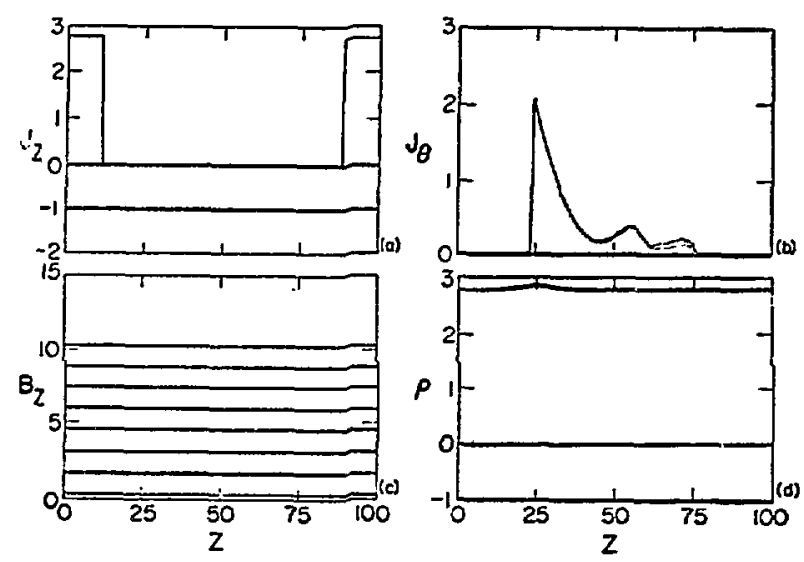

Fig. 23. Slug simulation terminated by $z_{o}$ and including the absorber, $t=450 \omega_{\mathrm{p}}^{-1}$ and $\tau=25 \omega_{\mathrm{p}}^{-1}$, (a) through (d) as in Fig. 19.
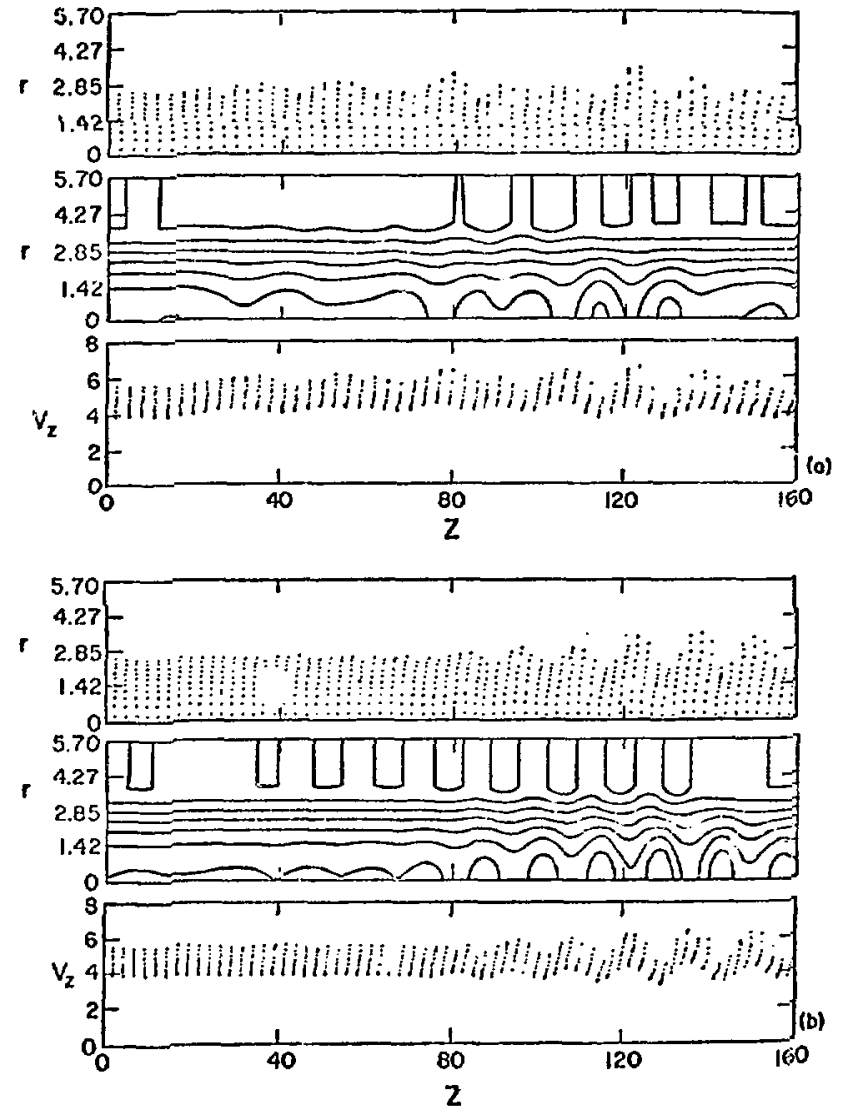

F1g. 24. Fuli particie simulations. The upper graph in both (a) and (b) is a plot of particle positions in the $r-z$ plane. The middle graph is a contour plot of the potential $\phi$ in the $r-z$ plane and lower graph in each is a phase space plot of the z-component of particle velocity versus axial position. Frame a) is an unterminated simulation at $t=1248 \omega^{-1}$ and $\tau=300$. Prame b) is a simulation terminated in $\mathrm{Z}_{\mathrm{o}}$ at $t=1155 \omega_{\mathrm{p}}^{-1}$ and $\tau=200 \omega_{\mathrm{p}}^{-1}$. In the contour plots for $\phi$, the innermost contour (those nearest $r=0$ ) have the value $\phi=-3.214$ and the contour interval is +0.57 . 
[To be submitted for publication in revised form.]

\section{APPENDIX I}

MULTIDIMENSIONAL THEORY OF THE INHOMOGENEOUS

BEAM-PLASMA INSTABILITY

Wi1liam R. Shanahan

ABSTRAC'

A combined analytic and numerical study of the effect of a plasma density gradient on the beam-plasma instability is presented. After a thorough discission of the qualitative aspects of this problem, emphasis is placed on the developinent of a theory which can reveal the interplay between the gradjent and the multidimensional aspects of the instatility. Using fluid equations, a differential equation is derived for the electrostatic potential. An integral representation for the solution of this equation is aerived and its asymptotic evaluation presented. Explicit comparison is made between this asymptotic result and a direct numerical integration of the basic equations. The predictions of the theory are presented for the two-dimensional case, both for the case in which the beam propagates along the direction of the gradient and for that in which the beam is propagating at an angle to this direction. Some brief remarks are made concerning the fully three-dimensional case. 


\section{INTRODUCTION}

The availability of intense relativistic electron beams has led to both linear ${ }^{1,2}$ and nonlinear ${ }^{3,4}$ studies of the instability which results when such a beam passes through a background plasma. Virtually all of these studies have been restricted to the idealized case in which the background plasma is considered to be infinite and uniform. However, in practical situations, such as those encountered in experiments designed to examine the possibility of employing relativistic electron beams to provide supplemental plasma heating, one is usually faced with the existence of various sorts of gradients. It therefore becomes imperative to evaluate the effects of such gradients on the collective beam-plasma interactions. The purpose of this paper is to initiate such an evaluation for the linear theory. A complete understanding of the linear theory is a necessary prerequisite to a study of the more practically relevant nonlinear thoery.

We consider in this paper what is in many respects the simplest nontrivial problem of this type, namely, that of the effect of a background plasma density gradient on the we11-known beam plasma instability. A fluid description is employed, and we restrict the discussion to the electrostatic approximation. However, a fully relativistic fluid equation of motion is used for the beam. The limitation imposed by the electrostatic approximation is not expected to be a serious one, for previous work on the uniform theory has demonstrated that the electrostatic portion of the interaction plays a ciuminant role even for relativistic beams. The geometry considered is sufficiently general to Include the possibility of both beam and wave propagation in directions other than that of the density gradient. When the beam is propagating at an angle to the gradient, the problem is fully three dimensional, since waves can now propagate either in the plane formed by the direction of bram propagation and 
that of the gradient or at an angle to that plane. Although we shall have little to say explicitly concerning this most general situation, interesting effects also arfse in more restricted cases which are easier to analyze both n. derically and analytically. Indeed, the interplay between the effects of the relativistic mass variation and those of the gradient form a principal theme of this paper.

A one-dimensional theory of an electron beam interacting with an inhomogeneous medium has previously been developed by Schmidt. ${ }^{5}$ We believe that the present work offers a number of extensions and advantages. Firstly, and most obviously, the present work includes multidimensional effects. A relativistic theory yields other than trivial differences from the nonrelativistic theory only when such effects are included. Since the most interesting practical applications involve relativistic beams, it is clearly important to have available a multidimensional theory. Secondly, Schmidt's theory rests upon an expansion in both configuration and wave number spaces of the dielectric function of the background plasma, which is taken from the uniform theory. It is often difficult to assess the validity of such expansions, and inasmuch as the expansions are effected in both real and wave-number space, the conditions of validity may even be contradictory. Further, sucii expansions require one to choose a point about which to expand, and the choice may be somewhat arbitrary. In the present work, by contrast, no such expansions are effected, and no results of the uniform theory are built into the foundations of theory. Rather, the uniform theory is recovered as a trivial specialization of the general case. Finally, we present a somewhat more careful asymptotic evaluation of the integral representation of the solution to the equation for the electrostatic potential. Against these advantages, one must set the fact that 
Schmidt's formalism is able to accomodate gradients of various sorts, whereas we are restricted here to density gradients. However, the advantages enumerated above coupled with our ability to make detailed comparisons between analytic and numerical computations seem to provide sufficient compensation for this restriction.

In addition to the electrostatic approximation, there are a number of other approximations which we make, the removal of which must await further study. It is well to state these at the outset. Firstly, we ignore complete1y the dynamics of the ion component of the background. For the relatively high frequency instabilities which are of principal importance for relativIstic beam-plasma interactions, this appears to be a good approximation. Although the inclusion of the ion dynamics does lead to the existence of modes which can be driven unstable, particularly by nonrelativistic electron beams, the growth rates of such unstable modes is usually negligible compared to these considered here. Secondly, we neglect any velocity spread in the beam. For relativistic electron beams, this appears to be a most reasonable assumption, while for nonrelativistic beams it may or may not be justifiable, depending on the circumstances. This is perhaps an appropriate point at which to remark that throughout the entire discussion we retain a finite temperature for the background plasma. For the relativistic case, where beam energies are typically much greater than plasma thermal energies, this may seem like a needlass complication, and the cold plasma approximation has been employed in discussions of the uniform linear theory. ${ }^{1}$ The reason for retainIng a finfte temperature here may be seen readily by recalling that a cold, untform plasma free of external fields is completely dispersionless, all wavelengths exhibiting the plasma frequency determined by the density. If a density gradient is introduced, each portion of the plasma vibrates 
independently at the plasma freclency determined by the local value of the density. The simple normal modes of the uniform system are thereby replaced by a continuum of modes. Efforts to interpret this singular situation have been extensively discussed in the literature, 6,7 but these difficulties are Irrelevant to our purposes. They may be simply eliminated by the physically motivated use of a finite temperature.

A further limitation in our work is that we consider only that portion of the collective beam-plasma interaction which is concerned entirely with Instability, and neglect completely that aspect which is concerned with the establishment of a return current. It is well known, however, that such a return current can play a significant role, particularly in plasma heating. In discussions of the uniform theory, the return current is also usually Ignored, the reason being that for realistic beam-to-plasma density ratios the velocity of the return current is negligible compared to that of the beam. For the inhomogeneous case, however, some care must be required. It is possible that in low density regions the return current could qualitatively affect the nature of the instability. In particular, we will assume throughout that the instability under consideration is convective. It is conceivable that in some portions of the density gradient the return current renders the interaction more akin to the two-stream instability, which is absolute in its space-time behavior. ${ }^{8}$ This is particularly possible for nonrelativistic beams. However, a full resolution of this question requires a treatment which retains the effects of the self-magnetic field of the beam, and which considers self-consistently the effects of current neutralization and instability. The combination of self-field and inhomogeneity seem to make this problem most suitable for numerical analysis, and we do not examine it further here. It Is assumed throughout the work, however, that the beam is both electrostatically and magnetically neutralized. 
Perhaps the most serious limitation of the present analysis from the point of view of its applicability to experiments is the omission of the effects of an external magnetic field. The presence of a magnetic field not only modifies the behavior of the modes considered here but also introduces new modes. Based upon analogy with the uniform theory, we anticipate that there will be regions of parameter space in which these new modes will be driven more strongly unstable than those examined below. It seems a straightforward, albelt tedious, task to extend the analysis presented here to the case of an inhomogeneous plasma immersed in a uniform magnetic field. This will be dealt with elsewhere. The problem that one would really like to address, however, is that in which both the density and the magnetic field possess gradients. This is by far the most common situation that one finds in practice. For example, if an electron beam is injected into a plasma confined in a magnetic mirror, one is faced with a situation in which the plasma density increases as the magnetic field decreases. Such problems seem not to be amenable to a straightforward generalization of the procedure employed here. The reason is that the tendency of beam electrons to follow field lines introduces a two-dimensional inhomogeneity into their velocity. This problem is probably best addressed through numerical methods, or, perhaps, through analysis based upon a local expansion. Hopefully, the work presented here vill provide a steppingstone to the treatment of such more realistic problems.

The paper is organized as follows. In Section II we display the equations upon which this work is based and derive from them a sixth order differential equation for the electrostatic potential which is the principal object of the analytic portion of the study. These equations have been Fourier analyzed in time, and we are therefore concerned here with the spatial structure of the 
normal modes of the system. No attention is paid to the equally important "temporal" problem which seeks to determine the fate of a localized pulse propagating through the system. In Section III, an integral representation is derived for the solution of this equation, and the results of an asymptotic evaluation presented. In this section we also discuss some features of the numerical methods employed here. The methods themselves are quite standard and do not require special discussion, but a delicacy arises in their implementation for the problem discussed here. Section IV is devoted to a presentation of results for the two-dimensional theory. An explicit comparison between the analytic expression derived in the previous section and the results of a direct numerical solution is given. It will be evident that the analytic results provide a quite adequate description of the principal effects of a density gradient on the beam-plasma instability. In Section $V$, we make a few brief remarks concerning the fully three-dimensional problem. Finally, in Section VI, we summarize our findings. 


\section{BASIC EQUATIONS}

In this section we derive an equation for the electrostatic putential which will form the basis for the analytic portion of our work. The tundamental fluid equations to be employed are the equations of continuity for plasma and beam species, respectively:

$$
\begin{aligned}
& \frac{\partial n_{p}}{\partial t}+\nabla \cdot\left(n_{p} v_{p}\right)-u \\
& \frac{\partial n_{b}}{\partial t}+\nabla \cdot\left(n_{b} \vec{v}_{b}\right)=0
\end{aligned}
$$

the nonrelativistic equation of motion for the background plasma electsuns:

$$
\left(\frac{\partial}{\partial t}+\vec{v}_{b} \cdot \nabla\right) \vec{v}_{p}=\frac{e}{m} \nabla \phi-\frac{\theta^{2}}{m} \frac{\nabla n_{p}}{n_{p}}
$$

the relativistic version of this equation for the beam piscicles:

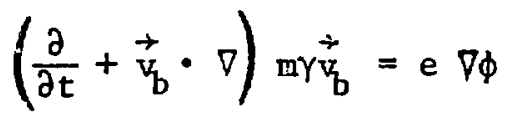

and Poisson's equation:

$$
\nabla^{2} \phi=4 \pi e\left(n_{p}+n_{b}\right)
$$

We have here chosen $e>0$, and $\theta$ is the thermal velocity of the background plasma. Slnce nonlinear effects are not examined here, we next proceed to IInearize these equations. Explicit time dependence will be removed through Fourler analysis, as will the spatlal dependence in the directions perpendicular to that of the gradient. Thus, for all quantities $\psi(x, y, z, t)$, one may write

$$
\psi(x, y, z, t)=\int d k_{y} d k_{z} d \omega \hat{\psi}\left(x, k_{y}, k_{z}, \omega\right) e^{-i \omega t+1 k_{y} y+i k_{z} z}
$$


where $k_{y}$ is the component of the perpendicular wave-vector $k_{1}$ which 1 ies in the plane formed by the directions of the gradient and that of beam propagation (the "plane of incidence") while $k_{2}$ is the component normal to this plane. Dropping the caret on transformed quantities, the resulting linearized equations are:

$$
\begin{aligned}
& -1 \omega n_{p}^{(1)}+\frac{d}{d x}\left(n_{p}^{(0)}(x) v_{p x}^{(1)}\right)+i n_{p}^{(0)}(x) \vec{k}_{1} \cdot \vec{v}_{p l}^{(1)}=0 \\
& D n_{b}^{(1)}+n_{b}^{(0)} \frac{d v_{b x}^{(1)}}{d x}+i \vec{k}_{1} \cdot \vec{v}_{f 1}^{(1)}=0 \\
& -i \omega v_{p x}^{(1)}=\frac{e d \phi}{m} \frac{d x}{d x}-\frac{\theta^{2}}{n_{p}^{(0)}} \frac{d n_{p}^{(1)}}{d x}+\theta^{2} \hat{L} \frac{n_{p}^{(1)}}{n_{p}^{(0)}} \\
& -\omega \vec{v}_{p 1}^{(1)}=\frac{e}{m} \vec{k}_{1} \phi-\theta^{2} \frac{\vec{k}_{1} n_{p}^{(1)}}{n_{p}^{(0)}} \\
& D\left[\left(m \gamma+m \gamma^{3} \frac{v_{x}^{(0) 2}}{c}\right) v_{b x}^{(1)}+m \frac{\gamma^{3} v_{b x}^{(0)} v_{b y}^{(0)}}{c^{2}} v_{b y}^{(1)}\right]=e \frac{d \phi}{d x} \\
& D\left[\frac{m \gamma^{3} v_{b x}^{(0)} v_{b y}^{(0)} v_{b x}^{(1)}}{c^{2}}+\left(m \gamma+\frac{m \gamma^{3} v_{b y}^{(0) 2}}{c^{2}}\right) v_{b y}^{(1)}\right]=i e k_{y} \phi \\
& D\left(m \gamma v_{b z}^{(1)}\right)=\text { iek }_{z} \phi \\
& \frac{\mathrm{d}^{2} \phi}{d \mathrm{x}^{2}}-\mathrm{k}_{1}^{2} \phi=4 \pi e\left(\mathrm{n}_{\mathrm{b}}^{(1)}+\mathrm{n}_{\mathrm{p}}^{(1)}\right)
\end{aligned}
$$

Equations (7)-(14) have been arranged to correspond to Equations (1)-(5). We have Introduced the notation 


$$
\hat{\mathrm{L}}=\frac{1}{n_{p}^{(0)}(x)} \frac{d n_{p}^{(0)}(x)}{d x} \text {, }
$$

the logarithmic derivative of the zero-order density profile. The term in (9) containing this quantity arises from the last term on the right-hand side of (3) through the following sequence of linearizations:

$$
\begin{aligned}
\left(\frac{1}{n_{p}} \frac{d n_{p}}{d x}\right)^{(1)} & =\left[\frac{1}{n_{p}^{(0)}+n_{p}^{(1)}} \frac{d}{d x}\left(n_{p}^{(0)}+n_{p}^{(1)}\right)\right] \\
& =\left[\frac{1}{n_{p}^{(0)}}\left(1-\frac{n_{p}^{(1)}}{n_{p}^{(0)}}\right) \frac{d}{d x}\left(n_{p}^{(0)}+n_{p}^{(1)}\right)\right] \\
& =\frac{1}{n_{p}^{(0)}} \frac{d n_{p}^{(1)}}{d x}-\frac{n_{p}^{(1)}}{n_{p}^{(0)}} \hat{L} .
\end{aligned}
$$

Advantage has been taken of the symmetry of the background plasma with respect to the directions perpendicular to the gradient to write Eq. (10). However, for the beam equation of motion the differences among the various directions are sufficiently strong to justify writing its components explicitly, Eqs. (11)-(13). In these equations we have also introduced the convenient notation:

$$
D=-i \omega+1 k_{y} v_{b y}^{(0)}+v_{b x}^{(0)} \frac{d}{d x},
$$

the convective derivative.

Equations (11) and (12) may be algebratcally manipulated to yield a more useful pair of differential equations involving $v_{b x}^{(1)}$ and $v_{b y}^{(1)}$ separately. The 
Intervening elementary steps yield:

$$
\begin{aligned}
& D v_{b x}^{(1)}=\frac{e}{m \gamma}\left[\frac{1}{\gamma^{2}}+\left(\frac{v_{b y}^{(0)}}{c}\right)^{2}\right] \frac{d \phi}{d x}-i \frac{e}{m \gamma} \frac{v_{b x}^{(0)} v_{b y}^{(0)}}{c^{2}} k_{y} \phi \\
& \text { (1) } \\
& D v_{b y}^{(1)}=i \frac{e}{m \gamma}\left[\frac{1}{\gamma^{2}}+\left(\frac{v_{b x}^{(0)}}{c}\right)^{2}\right]_{y} \phi{ }^{(1)}-\frac{e}{m \gamma} \frac{v_{b x}^{(0)} v_{b y}^{(0)}}{c^{2}} \frac{d \phi}{d x} .
\end{aligned}
$$

Our next step in the derivation of a differential equation for $\phi$ involves the elimination of the perturbed beam and plasma velocities from the above equations. For the plasma velocity perturbations, this is a trivial matter, since these are given explicitly by Eqs. (9) and (10). Substituting into (7), we obtain an equation involving $\phi$ and $n_{p}^{(1)}$

$$
\begin{gathered}
\theta^{2} \frac{d^{2} n_{p}^{(1)}}{d x^{2}}-\theta^{2} \hat{L} \frac{d n_{p}^{(1)}}{d x}+\left(\omega^{2}-k_{1}^{2} \theta^{2}\right) n_{p}^{(1)} \\
-\frac{e}{m} n_{p}^{(0)} \frac{d^{2} \phi}{d x^{2}}+n_{p}^{(0)} k_{1}^{2} \phi=0
\end{gathered}
$$

Examination of En. (8) together with Eqs. (17) and (18) reveals that the corresponding elimination of $v_{b}^{(1)}$ may be effected by operating on (14) with the operator D. After suitable expansions and regrouping of terms, this process ylelds: 


$$
\begin{aligned}
v_{x}^{(0)}= & \frac{d^{2} n_{b}^{(1)}}{d x^{2}}-2 i\left(\omega-k_{y} v_{b y}^{(0)}\right) v_{x}^{(0)} \frac{d n_{b}^{(1)}}{d x}-\left(\omega-k_{y} v_{b y}^{(0)}\right)^{2} n_{b}^{(1)} \\
& +n_{b}^{(0)} \frac{e}{m \gamma^{3}}\left[\left(1+\frac{v_{b y}^{(0) 2} \gamma^{2}}{c^{2}}\right) \frac{d^{2} \phi}{d x^{2}}-2 i \gamma^{2} \frac{v_{b x}^{(0) 2} v_{b y}^{(0)}}{c^{2}} k_{y} \frac{d \phi}{d x}\right. \\
& -k_{y}^{2}\left(1+\frac{v_{k x}^{(0) 2}}{c^{2}} \gamma^{2}\right) \phi-\gamma^{2} k_{z}^{2} \phi=0
\end{aligned}
$$

Equations (19) and (20), together with Poisson's Equation (14), constitute a system of three, coupled linear differential equations. This system of equations is, in fact, the most convenient form of our basic equations for numerical analysis. However, for purely analytic purposes, It is more useful to proceed to eliminate $n_{p}^{(1)}$ and $n_{b}^{(1)}$ and to obtain an equation for $\phi$ alone. Equations (19) and (20) indicate that this may be accomplished by operating on both sides of (14) with the product operator

$$
\left(\theta^{2} \frac{d^{2}}{d x^{2}}-\hat{L} \theta^{2} \frac{d}{d x}+\omega^{2}-k_{1}^{2} \theta^{2}\right) D^{2} \text {. }
$$

Introducing the standard notation $\omega_{p}^{(0) 2}=4 \pi n_{p}^{(0)} \frac{e^{2}}{m}$, the square of the local value of the plasma frequency, we have:

$$
\begin{aligned}
\left.\left(\theta^{2} \frac{d^{2}}{d x^{2}}-\hat{L} \theta^{2} \frac{d}{d x}+\omega^{2}-\theta^{2} k_{1}^{2}\right)\right) D^{2}\left(\frac{d^{2}}{d x^{2}}-k_{1}^{2}\right) \phi+\frac{\omega_{b}^{(0) 2}}{r^{3}} \\
\quad \times\left[\frac{d^{2} \phi}{d x^{2}}-2 y \gamma^{3} \frac{v_{b x}^{(0)} v_{b y}^{(0)}}{c^{2}} k_{y} \frac{d \phi}{d x}-k_{y}^{2}\left(1+\frac{v_{b x}^{(0) 2}}{c^{2}} \gamma^{2}\right) \phi-k_{z}^{2} \gamma^{2} \phi\right) \\
-D^{2} \omega_{p}^{(0) 2}(x)\left(\frac{d^{2} \phi}{d x^{2}}-k_{1}^{2} \phi\right)=0
\end{aligned}
$$


Throughout this paper we shall be concerned only with density profiles of the simple linear variety:

$$
\omega_{p}^{(0) 2}(x)=\omega_{p}^{2}\left(1+\frac{x}{h}\right)
$$

Further, it is assumed that terms proportional to $(1 / \mathrm{h})^{2}$ may be ignored. Under these restrictions, the operators appearing in (21) may be expanded to give the desired equation for $\phi$ :

$$
\begin{gathered}
A \frac{d^{6} \phi}{d x^{6}}+B \frac{d^{5} \phi}{d x^{5}}+(C+D x) \frac{d^{4} \phi}{d x^{4}}+(E+F x) \frac{d^{3} \phi}{d x^{3}}+(G+H x) \frac{d^{2} \phi}{d x^{2}} \\
+(a+b x) \frac{d \phi}{d x}+(d+e x) \phi=0
\end{gathered}
$$

where the coefficients A, B, - , are given by the expressions:

$$
\begin{aligned}
& A=-v_{b x}^{(0) 2} \\
& B=-v_{b x}^{(0) 2}\left(2 i k_{0}+\hat{L}\right) \\
& C=-\left[v_{b x}^{(0) 2}\left(k_{0}^{2}+2 k_{1}^{2}-2 i L k_{0}\right)+\frac{\omega_{b}^{(0) 2}}{\gamma^{3}}\left(1+\frac{v_{b y}^{(0) 2}}{c^{2}} \gamma^{2}\right)\right] \\
& D=-\frac{v_{b x}^{(0) 2}}{\theta^{2}} \frac{\omega^{2}}{h}
\end{aligned}
$$




$$
\begin{aligned}
& E=v_{x}^{(0) 2}\left[4 i k_{0} k_{\perp}^{2}-\frac{2 \omega^{2}}{h \theta^{2}}+\hat{L}\left(k_{0}^{2}+k_{\perp}^{2}\right)\right]+\frac{\omega_{b}^{(0) 2}}{\gamma^{3}}\left(1+\frac{v_{b y}^{(0) 2}}{c^{2}} \gamma^{2}\right) \\
& \times\left(2 \frac{v_{b x}^{(0)} v_{2 y}^{(0)}}{c^{2}} k_{y}-\hat{L}\right) \\
& F=\frac{21 k_{0} v_{b x}^{(0) 2} \omega^{2}}{h \theta^{2}} \\
& G=v_{b x}^{(0) 2}\left(k_{\perp}^{4}+2 k_{0}^{2} k_{\perp}^{2}-2 i \hat{i} k_{0} k_{\perp}^{2}+\frac{2 k_{0} \omega^{2}}{\theta^{2} h}\right)+\frac{w_{b}^{(0) 2}}{\gamma^{3}} \\
& \times\left[2 i \hat{L} k_{0} \frac{v_{x}^{(0)} v_{y}^{(0)}}{c^{2}}-\left(k_{\perp}^{2}-\frac{\omega^{2}}{H^{2}}\right)\left(1+\frac{v_{y}^{(0) 2} \gamma^{2}}{c^{2}}\right)-k_{z}^{2} \gamma^{2}+k_{y}^{2}\left(1+\frac{v_{x}^{(0) 2}}{c^{2}} \gamma^{2}\right)\right] \\
& \mathrm{H}=\frac{\omega^{2}}{h \theta^{2}} \quad \mathrm{v}_{\mathrm{bx}}^{(0) 2}\left(\mathrm{k}_{0}^{2}+\mathrm{k}_{1}^{2}\right) \\
& a=i v_{x}^{(0) 2}\left(-2 k_{1}^{4} k_{0}+\frac{2 \omega^{2}}{h \theta^{2}} k_{1}^{2}-\hat{L} k_{1}^{2} k_{0}^{2}\right)+\frac{i \omega_{b}^{(0) 2}}{\gamma^{3}} \\
& \times\left[\frac{2 v_{b x}^{(0)} v_{b y}^{(0)}}{c^{2}} \gamma^{2}\left(k_{1}^{2}-\frac{\omega^{2}}{\theta^{2}}\right) k y+\hat{L} k_{z}^{2} \gamma^{2}+k_{y}^{2} \hat{L}\left(1+\frac{v_{b x}^{(0)}}{c^{2}} \gamma^{2}\right)\right] \\
& b=-2 i k_{0}^{2} v_{b x}^{(0) 2} \frac{\omega^{2}}{h \theta^{2}} \\
& a=-v_{b x}^{(0) 2} k_{\perp}^{2}\left(\frac{\omega^{2} k_{0}^{2}}{\theta^{2}}+\frac{2 i \omega^{2}}{\theta^{2}} \frac{k_{0}}{h}\right)+\frac{w_{b}^{(0) 2}}{\gamma^{3}}\left[k_{z}^{2} \gamma^{2}+k_{y}^{2}\left(1+\frac{v_{b x}^{(0) 2}}{c^{2}} \gamma^{2}\right)\right] \\
& e=-k_{1}^{2} \frac{k_{0}^{2} v b y w^{2}}{h \theta^{2}} \\
& k_{0}=\frac{w-k_{y} v_{b x}^{(0)}}{v_{b x}^{(0)}}
\end{aligned}
$$


In writing these expressions we have taken advantage of sur freedom to choose the origin of the coordinate system to put $\omega_{p}^{(0) 2}=\omega^{2}$. This choice simpiifies the formulas slightly and possesses a certain physical cogency, Inasmuch that the uniform theory would indicate that the spatial gain is greatest in this region. In the limit of vanishing gradient, one may Fourier transform in the $\mathrm{x}$-direction as well. In this case the problem is at most two-dimensional, and we are at liberty to set $v_{y}=0$. Upon so doing, and suitably rearranging terms, Eq. (23) easily yields the well-known dispersion relation for waves propagating in a beam-plasma system:

$$
k_{x}^{2}+k_{1}^{2}-\frac{\omega_{p}^{2}\left(k_{x}^{2}+k_{1}^{2}\right)}{\omega^{2}-\theta^{2}\left(k_{x}^{2}+k_{\perp}^{2}\right)}-\frac{\omega_{b}^{(0) 2} k_{x}^{2}}{\gamma^{3}\left(\omega-k_{x} v_{x}^{0}\right)^{2}}-\frac{\omega_{b}^{(0) 2_{k}{ }^{2}}}{\gamma\left(\omega-k_{x} v_{x}^{0}\right)^{2}}=u
$$

In this section we have for completeness retained terms proportional to $\hat{\mathrm{L}}$, the logarithmic derivative of the zero-order density profile. However, numerical investigation of the above equations shows that, at least for the magnitude of gradients considered here, such terms are of little quantitative consequence. Inasmuch that their.inclusion considerabiy complicates the formulae to be presented below, we shall henceforth no longer display such terms. 
III. INTEGRAL REPRESEN'TATION AND NUMERTCAL METHODS

Equation (24) is obviously not one of the standard equations of mathematical physics. Conceivably, it could be reiuced to such an equatiou by some suitable approximation procedure, such as that embodied in the W.K.B. method. However, part of the motivation of the present paper arises from a desire to avoid such approximations, at least in the initial stages of our formulation. We are, therefore, faced with the task of solving (24). One could possibly look for series solution of this equation, but the high order involved makes it likely that the recursion relation would be rather complicated and the resultant series not very illuminating. Further, a series solution only yields local information, whereas we are primariiy interested in the overall effect of the gradient on the instability. This last remark suggests that it might be more fruitful to look for an integral representation of the solution given in (24).

We therefore look for solutions to (24) of the form:

$$
\phi(x)=\int_{C} \hat{f}(k) e^{i k x} d k
$$

where the form of $\hat{\mathfrak{f}}$ is determined by the requirement that the integrand vanish when (27) is acted upon by the differential operator appearing in (24), while the contours of integration are dictated by the requirement that $f$ itself vanish at the end points. In order to carry through this determination, one zeeds the simple identities: 


$$
\begin{aligned}
& \frac{d^{n} \phi}{d x^{n}}=i^{n} \int_{C} f(k) k^{n} e^{i k x} d k \\
& x \frac{d^{n} \phi}{d x^{n}}=1^{n+1} \int_{C} \frac{d}{d k}\left[k^{n} \hat{F}(k)\right] e^{i k x} d k
\end{aligned}
$$

Substituting (27) into (24) and applying (28), we may easily obtain a simple first order differential equation for $\hat{\mathfrak{f}}$ :

$$
\begin{gathered}
\left(1 D k^{4}+F k^{3}-1 H k^{2}-b k+e\right) \frac{d \hat{f}}{d k}=\left[A k^{6}-1 B k^{5}-C k^{4}+i(E-4 D) k^{3}\right. \\
\left.+(G-3 E) k^{2}-i(A-2 H) k+b-d\right] \hat{\tilde{I}}
\end{gathered}
$$

With the aid of a partial fraction decomposition, one may straightforwardly, if tedlously, perform the integration implied in (27). The result is:

$$
\begin{aligned}
\hat{\mathbf{f}} & =c \exp \hat{c}\left[c_{3} k^{3}+c_{1} k-\frac{c_{-1}}{k-k_{0}}+c_{p} \ln \left(k^{2}+k_{1}^{2}\right)+c_{q} \ln \frac{k-1 k_{1}}{k+i k_{1}}\right. \\
& \left.+c_{r} \ln \left(k-k_{0}\right)\right]
\end{aligned}
$$

where the coefficients are given by:

$$
\begin{aligned}
\hat{c} & =\frac{\theta^{2} h}{v_{b x}^{(0) 2} \omega^{2}} \\
3 & =\frac{v_{b x}^{(0) 2}}{3}
\end{aligned}
$$




$$
\begin{aligned}
c_{1} & =3 v_{b x}^{(0) 2}\left(k_{1} k_{0}-k_{0}^{2}\right)+k_{1}^{2} v_{b x}^{(0) 2}-\frac{\omega_{b}^{(0) 2}}{\gamma^{3}}\left(1+\frac{v_{b x}^{(0) 2}}{c^{2}}\right) \\
c_{-1} & =\left.\frac{2 \omega_{b}^{(0) 2}}{\gamma^{3}\left(k_{0}^{2}+k_{1}^{2}\right)}\right|_{1} ^{2}\left[\frac{2 v_{b x}^{(0)} v_{b y}^{(0)}}{c^{2}} k_{y} k_{0}-k_{0}^{2}\left(1+\frac{v_{b y}^{(0) 2}}{c^{2}}\right)-k_{z}^{2} \gamma^{2}\right. \\
& \left.-k_{y}^{2}\left(1+\frac{v_{b x}^{(0) 2}}{c^{2}} \gamma^{2}\right)\right]+\left(1+\frac{v_{b y}^{(0) 2}}{c^{2}} \gamma^{2}\right) \\
& \times\left(\frac{\omega^{2}}{\theta^{2}} k_{0}^{2}+\frac{2 v_{b x}^{(0)} v_{b y}^{(0)}}{c^{2}} k_{0}^{3} k_{y}-k_{0}^{4}\right)+\left[k_{z}^{2} \gamma^{2}+k_{y}^{2}\left(1+\frac{v_{b x}^{(0) 2}}{c^{2}} \gamma^{2}\right)\right] \\
& \left.\times\left(\frac{\omega^{2}}{\theta^{2}}-k_{0}^{2}\right)+2 \frac{\omega^{2}}{\theta^{2}} k_{y} k_{0} \frac{v_{b x}^{(0)} v_{b y}^{(0)}}{c^{2}}\right) .
\end{aligned}
$$

and where $c_{p}, c_{q}$, and $c_{r}$ are complicated functions of the various parameters Involved, but which do not play a significant roll in the asymptotic result to be displayed below. Therefore, they are not displayed here. 
Two possible contours of integration are shom in Fig. 1. The required additional contours may be simply generated by successively rotating that figure through $\pi / 3$, except of course that those contours of type B must approach the pole from the appropriate half of the complex plane.

It is apparent that it is no easier to integrate exactly the integral generated by substituting (30) into (27) than it is to solve the original differential equation (30) exactly. However, our form of the solution (27) permits us to make meaningful approximations. In particular, as noted at the beginning of this section, we are really primarily interested in the overall effect of the gradient on the instability, rather than on its detailed local evolution. This effect may be studied if the form of $\phi$ at large $x$ is ascertained. The form of the solution (27) is ideal for the derivation of such an asymtotic expression.

At this point, it is tempting to follow the example of those who have investigated the collective interaction of an intense laser beam with a plasma by obtaining the asymptotic form of (27) through a straightforward application of saddle-point techniques. ${ }^{9}$ A crucial difference must, however, be noted between the present problem and that of parametric instabilities in an inhomogeneous medium. The exponent appearing in the integral representation (27), given explicitly by (30) and (31), contains a pole in the variable of integration, while the corresponding exponent in the case of parametric instabilities contains no such pole. The appearance of such a pole In the present problem should not be too surprising, since such "resonant denominators" are a characteristic feature of beam-plasma instability theory. However, its appearahce does indicate that one must exercise care in obtaining an asymptotic form of $\phi$. The reason is that the integral, 
as it now stands, is not in the standard form for the application of saddle point methods. A purely pedestrian approach would yield expressions for the saddle points which depend on the variable $x$. Although this need not in itself be disastrous, one murc nevertheless insure that as $x$ approaches the asymptotic regime, the saddle points neither coalesce, nor merge with the pole. Otherwise, the expansions in which one must indulge in applying the saddle-point method become invalid. Unfortumately, in the present case, application of the usual procedure yields two saddle points which merge through the pole as $x \rightarrow \infty$. A similar situation has been noted in the initial value problem for a relativistic plasma. ${ }^{10}$ As in that case, the integral representation must be subject to several manipulations before an asymptotic result may be extracted. Here we only give the final result:

$$
\phi(x)_{x \rightarrow \infty} \exp \Gamma \sqrt{x}
$$

with $\quad \Gamma=2 \sqrt{\left|c_{-1}\right|}$

In the following sections the foregoing formula for $\Gamma$ will be analyzed to extract information regarding the effect of a background density gradient on the multidimensional properties of the beam-plasma instability. Naturally, it is desirable to gain some confidence in our analytic result. In order to gain such confidence, the set of three, coupled second order differential equations consisting of Eqs. (14), (19), and (20) were solved numerically to obtain the spatial structure of the normal modes of the system. At first sight, this would seem to be a perfectly straightforward problem. One would merely provide arbitrary values for the appropriate quantities and their 
derivatives at a given point and proceed to integrate away from that point. Unfortunately, in the present case such a procedure would lead to nonsense. The reason is not difficult to find and is unrelated to the presence of a density gradient. Note that the Erequency regime in which instability exists lies below the value of the local plasma frequency. Consequently, the beam mode of principal incerest to us coexists with two plasma modes which are but slightly affected by the presence of the beam. In the uniform limit, these modes possess imaginary wave vectors which are considerably larger than those belonging to the beam modes. Although we know physically that these modes are highly damped, to a single-point boundary value scheme such as that described above, one of these modes appears to be strongly amplified spatially. The apparent amplification is so strong that the beam mode is completely obscured. Therefore, one is faced with the problem of eliminating such unwanted modes.

Such problems have been encountered previous $\perp$ in other areas of plasma physics. Forslund ${ }^{11}$ has developed a two-point boundary-value numerica] code with which he and his collaborators ${ }^{12}$ nave employed to address various questions related to the propagation of an intense electromagnetic wave chrough an inhomogeneous plasma. This code permits one to eliminate any undesired mode by setting it to zerc at that end of the computational box at which, if not so constrained, it would have achieved its largest amplitude. The boundary conditions are established with aid of the dispersion relation provided by the uniform theory. The systems considered by Forslund et al. were sufficiently simple that many of the intervening steps could be performed analytically. In the present case, the large number of modes make it 
necessary to numerically automate the entire procedure. Accordingly, a program was written which supplied appropriate input to Forslund's code. It is the output of this code with which our analytic results are compared in the next section. 


\section{TWO-DIRENSIONAL THEORY}

The complexity of the foregoing formulae make it evident that 1ittle insight is to be gained by continuing to consider the most general case. Accordingly, in this section we turn to a consideration of particular cases. We here deal with phenomena which are strictly two-dimensional. Firstly. we discuss the case in which the electron beam is propagating along the density gradient. We refer to this case as "normal" propagation. Attention is then directed toward the situation in which the beam is propagating at an angle with the gradient. This will be referred to as "oblique" propagation. Only waves propagating in the plane formed by the directions of the gradient and that of beam propagation are examined in this section.

Most of the results to be reported here are based upon evaluation of our formula (32) for the asymptotic spatial gain. To insure that these results are not fictitious, we have made a direct comparison between the predictions of Eq. (32) and the results of the direct numerical integration of the basic equations. This comparison is shown in Fig. 2 for two different values of the gradient parameter $h$. This graph shows that the analytic expression provides a quite adequate description in the second half of the box, which may evidently be considered as being in the asymptotic regime. The frequency of this mode was chosen to be consistent with our conventions. The initial ratio of beam to plasma density was $1 / 3$. The density change from one end of the box to the other was, for the gentler gradient, slightly greater than a factor of two, while for the steeper case, it was approximately a factor of four. The slight distortion near the beginning of the box is an indication of the fact that we found it necessary to use a nonIInear profile in this region to reduce unwanted reflections. The 
unrealistically large value of the beam to plasma density ratio and the restricted range of density variation were imposed by the twin constraints of achieving sufficient accuracy and of not exhausting available computer storage. Nevertheless, this comparison serves its principal function of strengthening our confidence in the analytic results. The small finite temperature used in generating this comparison corresponds to a background thermal energy of approximately $100 \mathrm{eV}$.

Since Fig. 2 was generated with particular values of the relevant parameters, little information can be extracted from it. However, there is one conclusion that one may properly draw from it. This is that the dependence of the asymptotic growth rate on the gradient parameter $h$ is correctly prescribed by our formula for $\Gamma$. It is interesting to note, however, that our analytic result fails to accurately describe the normal mode structure, as revealed by numerical computation when values of $h$ much larger than those shown in Fig. 2 are used. This is to be expected. The reason is tha' the asymptotic variable is in reality $x / h$ rather than $x$ alone. Hence, for larger values of $h$, one must proceed to larger values of $x$ to enter the asymptotic regime. Presumably, agreement between analytic and numerical resul.ts could be regained by extending the length of the computational box. For the length considered here, the spatial gain is more nearly linear in $h$, rather than having a square root dependence as indicated by (31). These smeil gradients are of little practical interest, however.

Some general trends derived from our formula for $\Gamma$ will now be given. For comparison, we give in Fig. 3 the result of the uniform theory for the spatial gain as a function of $k_{1}$. Throughout this section we shall use $k_{1}$ 
as the independent variable, rather than the more intuitively appealing angle of propagation, for it is $k_{1}$ which is constant in an inhomogeneous medium and enters directly into the analysis. Fig. 3 mirrors the well-known fact that the nonrelativistic beam-plasma instability is largely a onedimensional affair, in the sense that the maximum growth rate is in the direction of propagation. For the relativistic case, however, this is certainly not true. As Fig. 3 clearly demonstrates, the growth rate first increases rapidly with $k_{1}$ then decreases slowly from this maximum. This is simply a consequence of the fact that it is easier to change the direction of the velocity of a relativistic particle than it is to change its magiitude.

We expect that the gradient will lead to an enhancement of the twodimensional character of the instability. This is verified in Fig. 4. Here we see that the growth rate for the nonrelativistic case is a somewhat gentler function of $k_{1}$ than in the uniform case. The dynamical tendency of the instability to grow along the direction of the beam propagation is compensated for partly by the presence of the gradient. For the relativistic beam, the effect is to enhance the two-dimensional character already apparent in the uniform case.

A somewhat more interesting case arises when the beam is permitted to propagate at angle to the density gradient. The nonrelativistic results are little modified, but the relativistic case shows a striking behavior. The growth rate, plotted again as a function of $k_{1}$ in Fig. 5, first increases as a function of $k_{1}$, then shows a sharp decrease, and then again rises. This behavior is easy to understand. The initial rise is due simply to the effect 
of not propagating directly along the gradient. The temporary decrease is an indication that the normal mode is sampling the effects of the longitudinal mass. The subsequent rise is due to the combined effect of the cause of the initial rise and of the transverse mass. 
v. THREE-DIMENSTONAL RESULTS

As noted in the introduction, the case of oblique beam propagation is in reality a three-dimensional problem, since a wave can prop?gate either in the "plane of incidence" or perpendicularly to that plane. It is clear that the growth rate must ultimately increase, since waves propagating normally to this plane feel no eftect of the gradient and little of that of the transverse mass. The only question remaining is whether this increase is monoto lic. We have made only preliminary investigations of this question. A sample calculation is shown in Fig. 6. The character of this function may be rationalized in a manner analogous to that given in the previous saction. 
VI. CONCLUSIONS

We have presented an analytic and numerical investigation of the effect of a density gradient on the beam-plasma instability. Emphasis has been placed on the derivation of a theory which can accomodate multidimensional effects. An analytic result for the asymptotic spatial gain was derived and compared with direct numerical integration of the basic equation. Based upon the agreement found in this comparison, we proceeded to numerically evaluate the spatial gain formula for various special cases. The principal effect of a gradient is to enhance the multidimensional character of the instability. Further parameter regimes could be investigated, but these should presimably await the call of particular applications.

\section{ACKNOWLEDGEMENTS}

I wish to thank B. Newberger and L. Thode for numerous helpful discussions throughout the course of this work. I am very grateful to D. Forslund for lending me a computer code with which to perform the numerical integrations, and for numerous helpful comments concerning its implementation. B. Godfrey and $E$. Lindman are also to be thanked for their assistance with the numerical portions of this work. Helpful comments by D. Dubois and J. KIndel are gratefully acknowledged.

This work was supported by the U. S. Energy Research and Development Administration. 


\section{REFERENCES}

1. B. Godfrey, W. Shanahan, and L. Thode, Phys. Fluids 18, 345 (1975).

2. R. L. Ferch and R. N. Sudan, Plasma Phys. 17, 905 (1975).

3. L. E. Thode and R. N. Sudan, Phys. Fluids 18, 1552 (1975).

4. L. E. Thode, Phys. Fluids 19, 831 (1976).

5. G. Schmidt, Phys. Rev. Lett. 33, 287 (1974).

6. E. M. Barston, Ann. Phys. 29, 282 (1964).

7. Z. Sedlacek, J. Plas. Phys. 5, 239 (3971), J. Plas. Phys. 6, 187 (1971).

8. R. J. Briggs, Electron Stream Interactions with Plasmas (MIT Press, Cambridge, MASS., 1964), Chapter 2.

9. M. N. Rosenbluth, C. S. Liu, and R. B. White, Phys. Rev. Lett. 31, 697 (1973).

io. B. Godfrey, B. Newberger, K. Taggart, IEEE Trans. Plas. Sci. PS-3, 185 (1975).

11. D. Forslund, Lectures presented at Summer School in Plasma Physics, Adarache France, 1974.

12. D. Forslund, J. Kindel, K. Lee, E. Lindman, and R. Morae, Phys. Rev. A11, 679 (1975). 


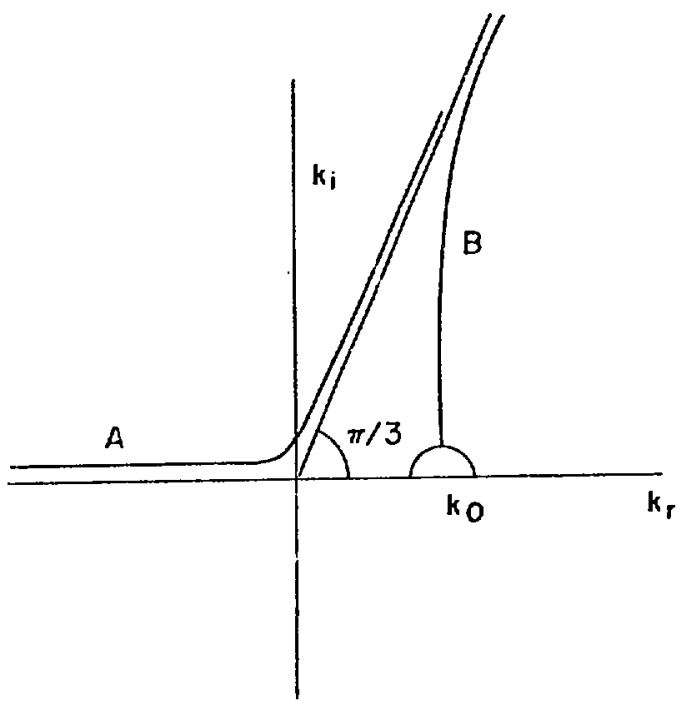

Fig. 1. Sample contours of integration.

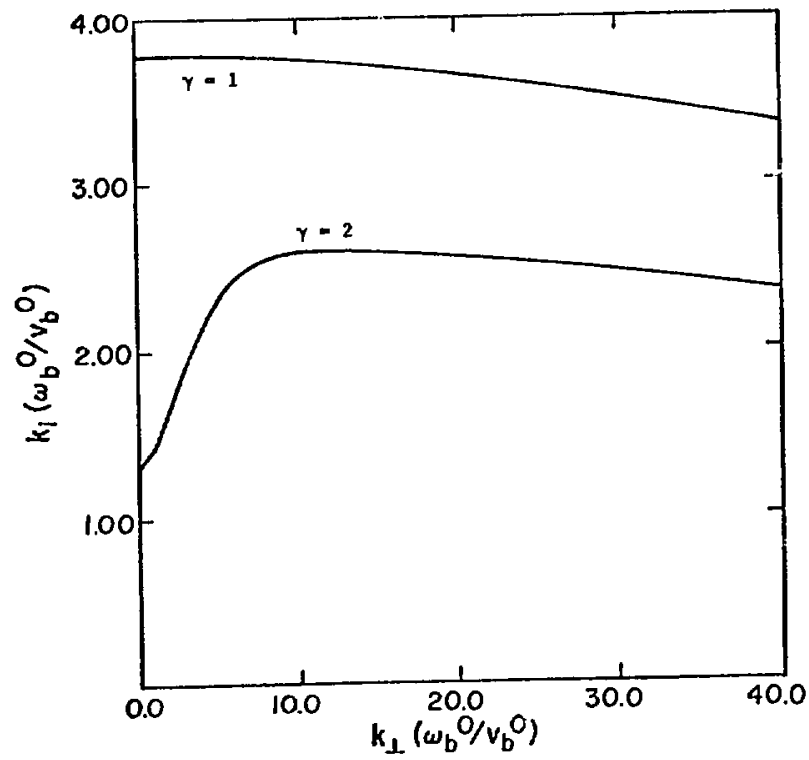

Fig. 3. Spatial gain as a function of $k_{\perp}$ for the uniform theory.

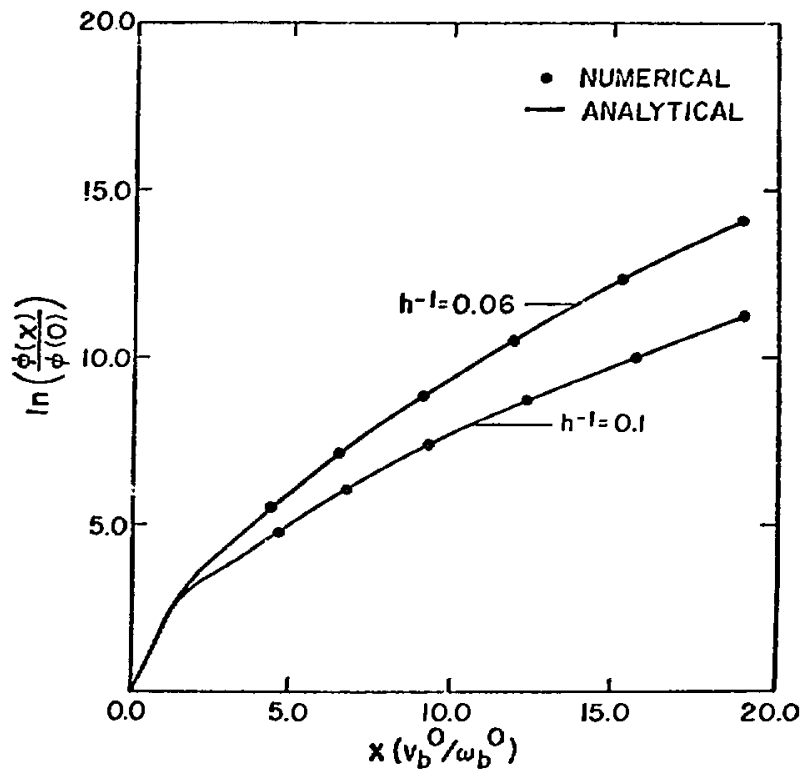

Fig. 2. Comparison of analytic and numeriral results for $k_{1}=3.1$ and $\gamma=2$.

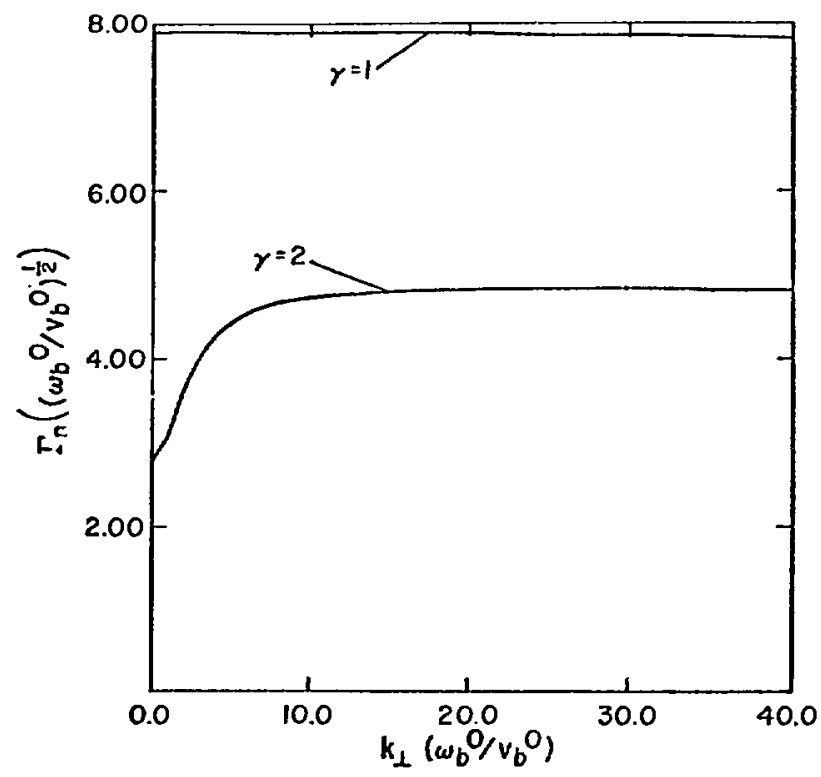

Fig. 4. Asymptotic spatial gain as a function of $k_{1}$ for $h^{-1}=0.06$. 


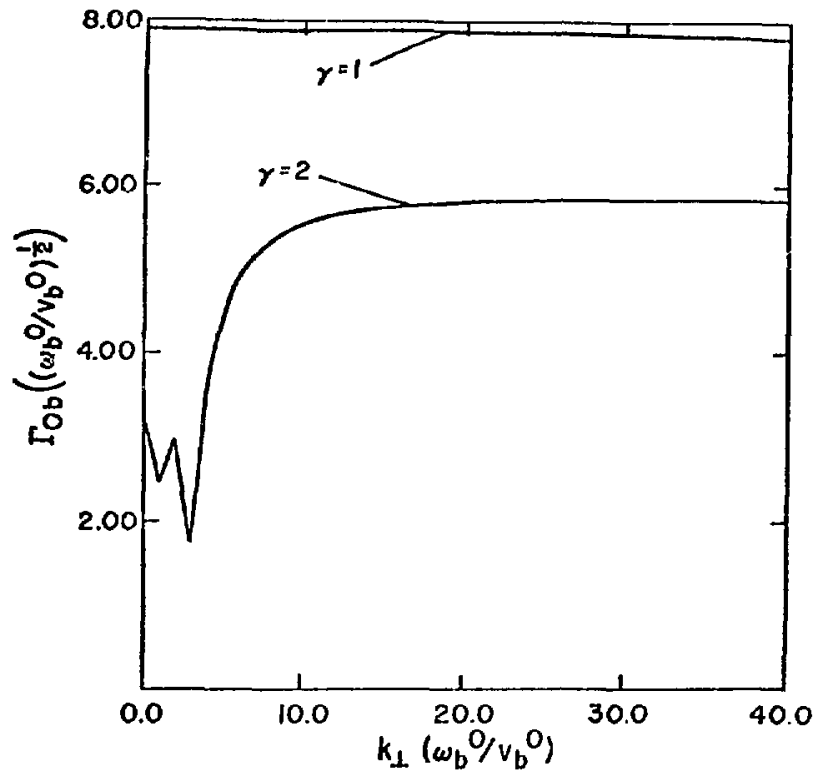

F1g. 5. Asymptotic spatial gain for an oblj.quely propagating beam.

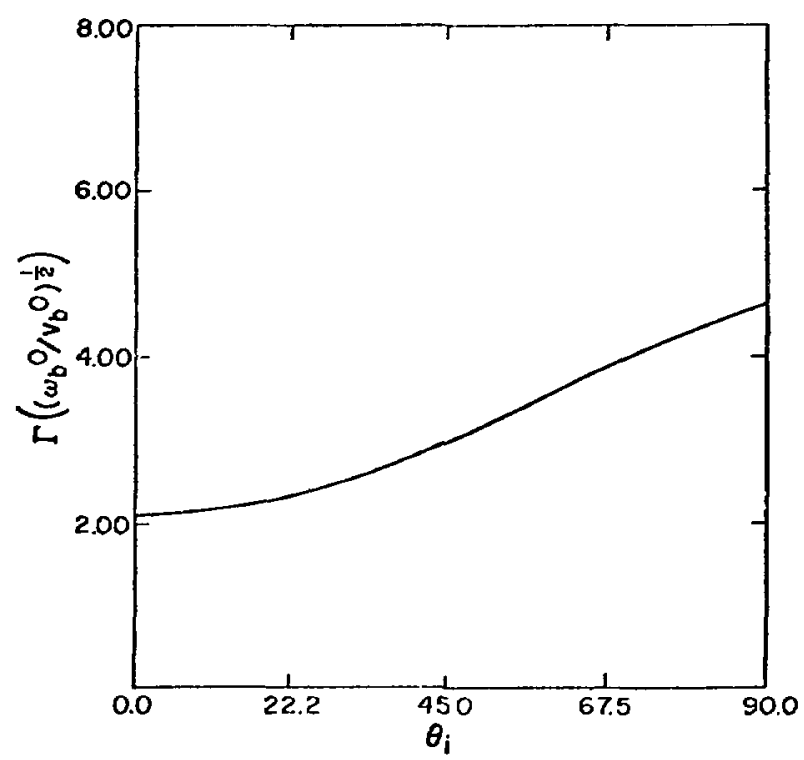

Fig. 6. Asymptotic spatial gatn for three-dimenstonal case. $\theta$ is the angle of inclination which the mode makes with the plane of incidence. 
[T: be published in IEEE Transactions on Plasma Science.]

\author{
APPENDIX J \\ LANGMUIR WAVE PHASE VELOCITY IN UNNEUTRALIZED BEAMS * \\ by
}

Brendan B. Godfrey

\begin{abstract}
We investigate the dispersion relation for long wavelength slow Langmuir waves in a relativistic particle beam propagating along a strong magnetic guide field in an evacuated metallic waveguide. For a large class of beam radial profiles, wave phase velocity drops to zero with infinite slope as beam current approaches the space-charge limit. This result is of importance to certain collective ion acceleration proposals.
\end{abstract}




\section{Introduction}

One of the more promising collective ion acceleration proposals 1 is the so-called Converging Guide Accelerator. ${ }^{2,3,4}$ Ions are first trapped in large amplitude Langmuir waves excited in an unneutralized intense relativistic electron beam streaming along a strong magnetic guide field and then accelerated by increasing the phase velocity of those waves. The phase velocity is controlled by propagating the beam In a waveguide of spatially varying radius. Physically, the change of wave velocity with waveguide radius arises from both the change in effective radial wavenumber ${ }^{5}$ and the change in beam kinetic energy ${ }^{6}$ due to the beam self-fields. 7,8

An important question confronting the Converging Guide Accelerator is whether sufficiently low phase velocities can be achieved for the trapping of stationary ions..$^{9}$ otherwise, some sort of fon pre-acceleration will be required. ${ }^{10}$ Recently, Briggs showed for a thin annular beam that the long wavelength slow Langmuir wave phase velocity is small only for beams currents near the space-charge limit, varying as $v_{W} \sim\left(I_{L}-I\right)^{1 / 2}$, where $I_{L}$ is the space-charge limiting current. ${ }^{11}$ In this situation, the phase velocity drops to zero with infinite slope, and we should expect that small wave velocities would be difficult to achieve in practice. It is, however, unlikely that acceleration experiments will emplay a thin hollow beam. So we wish to determine if this behavior of the phase velocity persists for more general beam current profiles.

Interestingly, Breizman and Ryutov independently obtained the hollow beam result, but with a different application in mind. ${ }^{8}$ They asked why the 
longitudinally constant equilibrium of a beam at the space-charge limit breaks up to form a virtual cathode ${ }^{12}$ when the current is increased infinitesimally, even though the beam particles retain some residual kinetic energy. Their speculation was that the vanishing Langmuir wave velocity gives rise to an ever increasing fluctuation level until spontaneous transition to a longitudinally varying equilibrium occurs. (Such an enhanced noise level has been observed in computer simulations of a thick annular beam. ${ }^{13}$ ) This explanation can be correct only if the phase velocity falls to zero at the space charge limit for all current profiles which permit virtual cathode formation.

In this note, we prove that $v_{w} \sim\left(I_{L}-I\right)^{1 / 2}$ near the space-charge limit for any beam in which the particle kinetic energy remains non-zero. It appears that most beam profiles, including all those explicitly studied analytically ${ }^{8,14}$ or numerically, ${ }^{13,15}$ fall into this catagory. In Sec. II, we define the space charge limit for beam propagation and obtain the behavior of the beam energy near that limit. We connect the variation of Langmuir wave phase velocity with the variation of beam energy in Sec. III, which leads to the general result just cited. Finally, we draw a few linited conclusions in Sec. IV. 
II. Space-Charge Flow

Consider a cylindrically symetric beam of arbitrary radial profile confined by a strong magnetic guide field in a metalic waveguide. The equilibrium electrostatic potential, normalized to the electron rest energy, is given far from the waveguide ends by

$$
\frac{1}{r} \frac{d}{d r} r \frac{d}{d r} \phi+\omega_{b}^{2}=0
$$

where $\omega_{b}^{2}$ is the local, radially dependent beam plasma frequency. (The speed of light has been set to unity.) In the strong guide field limit, current density is constant along cylinders of constant radius. Thus, we can relate $a_{b}^{2}$ in the waveguide to its value at the grounded injection plane (i.e., anode foil),

$$
\omega_{b}^{2}\left(1-\gamma^{-2}\right)^{1 / 2}=\omega_{b o}^{2}\left(1-\gamma_{0}^{-2}\right)^{1 / 2} .
$$

Here $\gamma$ is normalized particle energy. The subscript zero designates quantities at the injection point. Only $\gamma_{0}$ is necessarily independent of radius. Finally, conservation of particle energy gives

$$
r+\phi=r_{0}
$$

This closes the set of equations, defining the equilibrium in terms of the initial beam energy, current profile, and waveguide radius. 
This derivation is well known for, e.g., the case of a thin annular beam. 8,11 Proceeding with that calculation, one would solve Eq. (1) - (3) to obtain a nonlinear algebraic equation relating $\phi$ on axis to the total current. Setting $d I / d \phi$ to zero then defines the maximum, or space-charge limiting, current winch can propagate through the waveguide for the given beam and waveguide radii and beam injection energy. Note that $\gamma_{L}=$ $\gamma_{0}^{1 / 3}>1$ at the space-charge limit, indicating that the particle kinetic energy is non-zero.

It seems natural to employ this procedure for any fixed current profile, equating the derivative of the total current with respect to the potential on axis to zero in order to find the space charge limit. However, we do not in general know that $d I / d \phi=0$ even has a solution for physically meaningful values of $\phi$ and $I$. If it doesn't, then $\phi$ on axis presumably simply increases smoothly with I until it reaches the value $\gamma_{0}-1$. (From Eq. (2) and (3), $\omega_{b}^{2}$ then becomes singular at the center of the bean. Yet, if the singularity is integrable, it is not necessarily unacceptable.) The derivation that follows requires that $d I / d \phi=0$ have a physically realizable solution, and so we shall make that assumption. As noted in the Introduction, the assumption excludes no known space-charge flow equilibria. For beams profiles satisfying the assumption, particles retain a finite kinetic energy at the space-charge limit. Virtual cathode furmation must, therefore, be an abrupt and essentially discontinuou transition, in contrast to the excluded cases. 
It is straightforward to prove (see below) that

$$
\mathrm{d} \phi / \mathrm{dR} \rightarrow \infty \quad
$$

where $R$ is the waveguide radius, is an equivalent definition of the spacecharge limit. This asymptotic dependence will occur simultaneously for all radial values of $\phi(r)$. Intuitively, Eq. (4) better describes the Converging Guide Accelerator, in which beam current is fixed but waveguide radius changes adiabatically with position. Eq. (4) is, in any case, more convenient to work with here than is $d I / d \phi=0$, with $\phi \equiv \phi(0)$.

We begin by casting Eq. (1) into integral form so as explicitly to include the boundary conditions on $\phi$.

$$
\begin{array}{r}
\phi(r)=\int_{0}^{R} \omega_{b}^{2} K\left(r, r^{\prime}\right) r^{\prime} d r^{\prime} \\
K\left(r, r^{\prime}\right)= \begin{cases}\ln R / r, & r^{\prime}<r \\
\ln R / r^{\prime}, & r^{\prime}>r\end{cases}
\end{array}
$$

The integral in Eq. (5) depends implicitly on $\phi$ through Eq. (2) and (3). Using these two equations, we now differentiate Eq. (5), obtaining

$$
\begin{aligned}
& \frac{d \phi}{d R}=\int_{0}^{R} \frac{\omega_{b}^{2}}{r^{3}-r} \frac{d \phi}{d R} K\left(r, r^{\prime}\right) r^{\prime} d r^{\prime} \\
& +\frac{1}{R} \int_{0}^{R} \omega_{b}^{2} r^{\prime} d r^{\prime} .
\end{aligned}
$$


Taking $\omega_{b}$ and $\gamma$ as given for the moment, we can formally solve Eq. (7) by the standard techniques applicable to Fredholm equations. ${ }^{16}$

$$
\begin{gathered}
\frac{d \phi}{d K}=\left[1+\sum_{i} \int_{0}^{R} \frac{\omega_{b}^{2}}{\gamma^{3}-\gamma} \psi_{1}\left(r^{\prime}\right) r^{\prime} d r^{\prime} \psi_{i}(r)\left(\lambda_{i}-1\right)^{-1}\right] \\
\cdot \frac{1}{R} \int_{0}^{R} \omega_{b}^{2} r^{\prime} d r^{\prime},
\end{gathered}
$$

where $\lambda_{i}$ and $\psi_{i}$ are solutions to the Sturm-Liouville problem,

$$
\psi_{i}=\lambda_{i} \int_{0}^{R} \frac{\omega_{b}^{2}}{\gamma^{3}-\gamma} \psi_{i}\left(r^{\prime}\right) k\left(r, r^{\prime}\right) r^{\prime} d r^{\prime}
$$

Because by our earlier assumption $\gamma>1$ even at the space-charge limit, both $\lambda_{i}$ and $\psi_{i}$ must be well behaved functions of $R$ there. It follows that Eq. (4) can be satisfied only by $\lambda_{i} \rightarrow 1$ in Eq. (8) for some $i$ (probably, $i=1$ ). Now, from the basic theorems of Sturm-Liouville theory, ${ }^{17}$ we can show that

$$
\lambda_{1}-1 \sim\left(R_{L}-R\right) d \phi / d R
$$


as $R \rightarrow R_{L}$, the waveguide radius at which the space-charge limit is reached. Inserting Eq. (10) into Eq. (8), we immediately obtain the first of two important results,

$$
d \phi / d R \sim\left(R_{L}-R\right)^{-1 / 2}
$$

The variation of particle energy with waveguide radius follows immediately from Eq. (3).

Incidentally, had we proceeded directly from the definition $d I / d \phi=0$, $\phi \equiv \phi(0)$, we would have eventially reached $E q$. (9) with $\lambda_{i}=1$, showing the equivalency of that definition and Eq. (4). In addition, we can see from Eq. (8) that Eq. (4) must be satisfied for all $r$ at once. We note for use below that, for $R \approx R_{L}$, the second integral in Eq. (7) is bounded and can be dropped, giving Eq. (9) with $\psi_{i}=d \phi / d R$ and $\lambda_{i}=1$. 


\section{Langmuir Wave Phase Velocity}

The radial eigenfunction equation for linear perturbations on a

cylindrical cold fluid beam equilibrium in a strong guide field is 8

$$
\frac{1}{r} \frac{d}{d r} r \frac{d}{d r} E_{z}=\left(k^{2}-\omega^{2}\right)\left[1-\omega_{b}^{2} / \gamma^{3}(\omega-k v)^{2}\right] E_{z}
$$

Here, $\omega$ and $k$ are the frequency and longitudinal wavenumber of the normal mode. In the long wavelength $(k R \ll 1)$, low phase velocity $\left(\omega / k=v_{w} \ll 1\right)$ limit, Eq. (12) simplifies to

$$
\frac{1}{r} \frac{d}{d r} r \frac{d}{d r} E_{z}=-\omega_{b}^{2} / \gamma^{3}\left(v-v_{w}\right)^{2} E_{z}
$$

Now transforn this equation into integral form.

$$
E_{z}=\int_{0}^{R} \frac{\omega_{b}^{2}}{\gamma^{3}\left(v-v_{w}\right)^{2}} E_{z} K\left(r, r^{\prime}\right) r^{\prime} d r^{\prime}
$$

The similarity between Eq. (7) and (14) is striking. In fact, if we take $R \rightarrow R_{L}$ in Eq. (7) and $v_{w}=0$ it: Eq. (14), the two become identical. This proves that $v_{w}=0$ for some Langmuir wave in a beam at the space-charge limit. That $E_{z} \approx\left(R_{L}-R\right)^{1 / 2} \mathrm{~d} \phi / d R$ shows it is the fundamental (node free) Langmuir mode, which is of interest for the Converging Guide Accelerator. To obtain the dependence of $v_{w}$ on $R$ near $R_{L}$, we again invoke SturmLiouville theory, ${ }^{17}$ together with the observation that $\mathrm{dE}_{\mathrm{z}} / \mathrm{dR}$ is bounded by $d \phi / d R$.

$$
d v_{w} / d R \sim-d \phi / d R
$$


Combining Eq. (11) and (15) and iutegrating gives the second of our two main results,

$$
v_{P} \sim\left(R_{L}-R\right)^{1 / 2}
$$

as $R \rightarrow R_{L}$. 
IV. Conclusions

We have proven for a wide class of beain current profiles that the phase velocity of slow, long wavelength Langmuir waves drops to zero with infinite slope as the space-charge limit is appraoched. Although the preceding calculations dealt explicitly only with variations in waveguide radius, it is clear that similar results hold for variations in beam injected energy or total current. Therefore, it should prove very difficult in practice to achieve wave velocities sufficiently slow for convenient trapping of ions (without some injection accelerator) in the proposed Converging Guide Accelerator, ${ }^{2,3}$ at least for waves of linear amplitude. However, it is quite possible that non-linear effects in finite amplitude waves will be larger near the space-charge limit, precisely because smali changes in parameters can change linear wave velocities (and, indeed, the equilibrium) so dramatically. Preliminary experimental work tends to support this speculation. 4

It is worth noting that numerical solutions now underway of nonneutral beam linear theory in the cold fluid limit but with the infinite guide field constraint relaxed also show the strong variation of $v_{w}$ with $R_{L}-R_{0}$ 
* Work supported in part by the Energy Research and Development Administration and the fir Force office of Scientific Research.

1. For a recent review, see A. A. Kolomensky, Proc. IX Int. Conf. High inergy Accelerators (SLAC, Stanford, 1974), p. 254.

2. P. Sprangle, A. T. Drobot, and W. M. Manheimer, Prys. Rev. Lett. 36, 1180 (1976).

3. S. V. Yadavaddi, App1. Phys. Lett. 29, 272 (1976).

4. G. Gammel, J. A Nation, and M. E. Read, Bull. Am. Phys. Soc. 21, 1184 (1976).

5. A. W. Trivelpiece and R. W. Gould, J. Appl. Phys. 30, 1784 (1959).

6. B. B. Godfrey, W. R. Shanahan, and L. E. Thode, Phys. Fluids 18, 346 (1975).

7. L. S. Bogdankevich and A. A. Rukhadze, Sov. Phys. Uspekhi 14, 163 (1971).

8. B. N. Breizman and D. D. Ryutov, Nuc. Fus. 14, 873 (1974).

9. J. R. Thompson, unpublished.

10. J. A. Nation, J. Plas. Phys. 13, 361 (1975).

11. R. J. Briggs, Phys. Fluids 19, 1257 (1976)。

12. V. S. Voronin, Y. T. Zozulya, and A. N. Lebedev, Sov. Phys. Tech. Phys. 17, $432(1972)$.

13. R. J. Faeh1, R. B. Miller, and B. B. Godfrey, Bul1. Am. Phys. Soc. 21, 1165 (1976).

14. J. R. Thompson and M. L. Sloan, private communication.

15. B. B. Godfrey and L. E. Thode, Bul1. Am. Phys. Soc. 20, 1271 (1975).

16. F. B. Hildebrand, Methods of Applied Mathematics (Prentice-Hall, 1965), Ch. 3.

17. H. F. Weinberger, Partial Differential Equations (Blaisdell, 1965), Ch. 7 . 
[To be published in IEFE Transactions on Plasma Science.]

\author{
APPENDIX K \\ THE LOCALIZED PINCH MODEL AS A HIGH ENERGY ION \\ COTIECIIVE ACCELERATION MECHANISM* \\ by
}

Brendan B. Godfrey

\begin{abstract}
We present analytic and numerical calculations of the localized pinch model for ion collective acceleration by intense relativistic electron beams. These studies demonstrate the model's postulated synchronous acceleration of the ion clump and beam pinch. However, the configuration is found to be phase unstable, terminating the acceleration process before significant ion energy is achieved. Therefore, although the localized pinch model may be an appropriate explanation for some previous experimental observations of collectively accelerated Mev ions, it does not appear to be a suitable mechanism for high energy ion acceleration.
\end{abstract}




\section{INTRODUTION}

One of the first explanations proposed for the ion acceleration observed in many drifting intense relativistic electron beam experiments ${ }^{1}$ is the localized pinch model. ${ }^{2-5}$ According to this model, the electron beam propagates in Budker's for a neutral equilibrium, ${ }^{6}$ in which selfmagnetic field-induced pinching is exactly balanced by electrostatic field-induced radial expansion. For a low divergence beam, a background Ion density $n_{i}=\gamma^{-2} n_{e}$ is sufficient for force balance. No external guide magnetic field is required. The beam does not rotate. (Here, $\gamma$ is the electron energy in units of its rest mass, as conventionally defined relativistically.)

If, now, a clump of excess Ions arises as a result of, for instance, Ionization irregularities ${ }^{4}$ or instabilities, $\%, 8$ a localized pinch should form and the resulting electric fields give rise to ion acceleration. Moreover, because the pinch tends to form dormstream of the ion clump whereas the ions move toward the pinch, both pinch and clump might be expected to accelerate in synchronism, yielding high final lon energies. Figure 1 shows schematically the fon clump, beam pinch, and resulting electrostatic potential well.

There are, of course, several alternative explanations of experimentally observed collective ion acceleration, $1,9,10$ and a certain amount of controversy exists over which is to be preferred. It is important to emphasize, therefore, that the point of this paper is not to investigate the validity of the localized pinch model as an explanation of these 
experimental observations. Rather, it is directed at assessing whether the localized pinch mechanism can be employed in a controlled manner to achieve ion acceleration to hundreds of $\mathrm{MeV}$ per nucleon. Applications for such beams of light or heavy ions include controlled thermonuclear fusion, electro-nuclear breeding, basic nuclear physics, materials studies, and radiation therapy.

Three primary issues need to be resolved in determining whether the localized pinch model is useful to high energy ion acceleration: Can the force neutral equilibrium, which is quite delicate, be achieved in practice over adequate distances? Can one or more compact ion clumps be reliably produced? Can the ciump and resulting pinch accelerate together in a stable fashion? This article addresses only the last question. We find that the ion clump does not occupy a phase stable position in the local electrostatic well of the pinch. Rapid longitudinal spreading of the ion clump results, and acceleration terminates when the clump length somewhat exceeds the electron bedm diameter. Final ion energies are but a few times the electron initial kinetic energy. In Sec. II we derive from an envelope equation for the force neutral electron beam the pinch length, amplitude, and well depth. as functions of the electron beam parameters and the total excess charge of the ion clump. By balancing the electrostatic field of the pinch against the self-fleld of the clump, we find that $\nu / \gamma>1$ is necessary for clump equilibrium. (Here, $v$ is the normalized electron beam current, ${ }^{6}$ and $v / \gamma$ is evaluated within the metal guide tube after beam particles have given up energy to form self fields. The injection value could be somewhat smaller.) 
Sec. III contains results of self-consistent, two-dimensional (aperiodic cylindrical), relativistic, electromagnetic computer simulations of the localized pinch model. The first set of simulations tests the response of the electron beam to an ion clump of fixed position and dimenstons, thereby successfully corroborating analytic results obtained in Sec. IT. Ions in the clump are allowed to move in the seccnd set of simulations. It is here that we obtain our principal result: The clump is phase unstable, spreads rapidly, and dissipates before high ion energies can be achieved.

A discussion of the results and of possibilities for further research is given in Sec. IV. 


\section{ANALYTIC ESTIMATES}

The envelope equation for a low divergence, partially charge neutralized, relativistic electron bearn is 5

$$
a \frac{\mathrm{d}^{2} \mathrm{a}}{\mathrm{d} \mathrm{z}^{2}}=\frac{2}{\beta^{2}} \frac{\mathrm{v}}{\gamma}\left(\gamma^{-2}-\mathrm{f}\right)
$$

with $a$ the beam radius, $B$ the beam particle velocity, $v$ the beam current, and $\gamma$ the beam particle energy, all in dimensionless representation. The fractional charge neutralization, given in terms of the background ion and bean electron densities, is

$$
\mathbf{f} \equiv \mathbf{n}_{i} / \mathbf{n}_{\mathrm{e}}
$$

As noted in the Introduction, a partial neutralization $f=\gamma^{-2}$ gives force neutral equilibrium. ${ }^{6}$ If 6 locally exceeds this value, the beam pinches downstream, creating a potential well. Figure 1 illustrates this behavior, the heart of the localized pinch model.

Numerical integration of Eq. (1) for strong pinching seems impractical, because $\nu, \beta, \gamma$, and 6 depend on $a$ in a complicated fashion. Moreover, strong pinching often leads to violation of the laminar flow assumption implicit in the envelope equation. Some useful estimates of the potential well parameters may be obtained, however, by assuming the Ion background fixed (in which case $6 \propto a^{2}$ ) and linearizing Eq. (1). This leads immediately to a characteristic wavenumber for the pinch,

$$
k a=\frac{2}{\beta \gamma}\left(\frac{v}{\gamma}\right)^{1 / 2}
$$


The beam modulation amplitude can be estimated from Eq. (3) and the Coulomb scattering formula,

$$
\mathrm{k} \Delta a=-\frac{2}{\beta^{2}} \frac{\nu}{\gamma} \frac{Q_{1}}{Q_{e}},
$$

where $Q_{i}$ is the total excess charge in the ion bunch and $Q_{e}$ is the electron charge ili a volume $\pi a^{3}$. A more rigorous derivation of Eq. (4) appears iil Ref. 5.

For $\mathrm{ka}<1$, the potential well depth (measured in units of the electron rest energy) is satisfactorily approximated using space charge flow formulas, 11

$$
\phi_{\text {pinch }}=\frac{2 \gamma^{2}}{B}\left(\frac{\nu}{\gamma}\right)^{3 / 2} \frac{Q_{i}}{Q_{e}}
$$

If we take the radius of the ion clump to be of order $a / 2$, the optimal size based on simulation studies, the self-potential of the clump is roughly

$$
\phi_{\text {bunch }}=2 v \frac{Q_{1}}{Q_{e}} .
$$

Eqs. (5) and (6) together give

$$
\frac{\mathrm{ka}}{2} \phi_{\text {pinch }}=\frac{1}{\beta^{2}} \frac{\mathrm{v}}{\gamma} \phi_{\text {ion }} \text {. }
$$


In order that the ion bunch have a chance of holding together, the pinch field must dominate the ion stif field. Crudely, this demands $v / \gamma>1$ from Eq. (7). Note that the value of $v / Y$ at injection from the diode can be substantial?y lower, depending upon the amount of energy required to set up the beam equilibrium fields. 


\section{NUMERICAL SIMULATIONS}

The preceding analysis assumes a weak, laminar pinch and says little about the optimum shape or total sharge of the ion clump. Therefore, we performed a series of two-dimensional numerical simulations using CCUBE $^{12}$ configured in cylindrical coordinates to verify and expand upon the analytic estimates. In each of these simulations, $\gamma=3$ while $v / Y=1 / 2,1,2$. Both the ion background, density $n_{i}=n_{e} / 9$, and the Ion clump were held fixed in position. However, the rlump charge was increased adlabatically throughout each.simulation to investigate economically the variation of well depth with $Q_{i}$. From simulation to simulation different clump shapes and dimensions were employed.

The simulations show first of all that the ion clump should have characteristic dimensions (e.g. radius) comparable to about half the beam radius. A much smaller clump gives rise to a turbulent, irregular pinch and a reduced potential well depth. In addition, the self-potentiai of the clump is very large. On the other hand, the various parts of a much larger clump do not contribute coherently to pinch formation, again resulting in reduction of the electrostatic well depth.

For clumps of the optimal dimensions, simulation results are fairly accurately characterized by the analytic estimates of Sec. II, even for strong, somewhat non-laminar pinches. Figure 2 presents we11 depth as a function of excess ion charge for one such simulation with $v / \gamma=2$. Superimposed is the prediction of Eq. (5). Agreement is good until the we11 energy nears that of the unperturbed electron beam.

Using the best parameters from the static ion clump studies, we next performed a few simulations with mobile ions in the clump but with 
the background still fixed. As illustrated in Fig. 3, a strong pinch promptly forms, and the ions begin accelerating. Enfortunately, the process is found to be phase unstable: Ions at the front of the clump accelerate faster than those at the rear. The clump, therefore, elongates, the weli depth decreases, and acceleration eventually ceases. Figure 4 pictures this late time behavior. The corresponding distribution of hydrogen ion longitudinal velocities given in Fig. 5 indicates a maximum ion energy not greatly exceeding the electron energy. The fundamental problem here is that the beam begins pinching only as it passes the ion clump. Hence, it appears that the ions must always lie in the rearmost, phase unstable region of the pinch field. There is no obvious solution to this difficulty within the context of the localized pinch model. 
IV. CONCLUSIONS

The results presented demonstrate that, in agreement with the localized pinch model, a compact clump of excess ions within an intense relativistic electron beam in force neutral equilibrium gives rise to a strong pinch and that the pinch and ions accelerate synchronously. However, this configuration is unstable and, therefore, unsuitable for high energy ion acceleration.

One can think of various modifications which might improve upon the situation. Upstream modulation of the beam by external means, for instance, possibly could shift the relative phase of clump and pinch so as to produce phase stability. (Appropriately sweeping the modulation frequency as acceleration progresses is apt to be technically quite difficult.) Addition of a guide magnetic field opens a realm of novel possibilities. Varying this field longitudinally yields an arrangement reminescent of autoresonant ion acceieration. ${ }^{13}$ Bending the guide field and beam into a torus, on the other hand, may allow constructive interference of waves launched by the pinch with the pinch itself. ${ }^{14}$

That our simulations involve an immobile ion background precludes any beam-ion instability. ${ }^{8}$ Such instabilities are likely sources of ion clumps in the first place and would have provided an initial ion velocity. 7 Some backgiound ions might also have been picked up by the accelerating clump to compensate for ions lost from the rear. These two effects could boost the final. energy of ions in the clump to three or four times the electron energy, enough for consistancy with MeV ion acceleration observations but insufficient to change the primary conclusion of this report. 


\section{REFERENCES}

1. G. Yonas, Part. Accel. 5, 81 (1973), and references therein.

2. S. Putnam, Phys. Rev. Lett. 25, 1129 (1970).

3. S. Putnam, IEEE Trans. Nuc. Sci. NS-18(3), 496 (1971).

4. S. Putnam, "Theoretical Studies of Intense Relativistic Electron Beam-Plasma Interactions," PIFR-72-105 (Physics International Company, San Leandro, 1972), Ch. 4.

5. A. A. Irani and N. Rostoker, "Theory of the Synchronized Pinch," UCI-TR-76-16 (University of California, Irvine, 1976).

6. G. I. Budker, Atom. Ener. $1, y$ (1956) [Sov. J. Atom. Ene:. 1, 673 (1956)] .

7. B. B. Godfrey and L. E. Thode, IEEE Plas. Sci. 3, 201 (1975).

8. K. V. Khodataev and V. N. Tsytovich, Fiz. P1azmy 2, 301 (1976) [Sov. J. Plas. Phys. 2, 164 (1976)].

9. C. L. Olson, Particle Acce1. 6, 107 (1975), and references therein.

10. C. L. Olson, Phys. Fluids 18, 585 and 598 (1975).

11. B. B. Godfrey, IEEE Plas. Sci., to be published.

12. B. B. Godfrey and W. R. Shanahan, in N. C. Cooper (ed.), "Theoretical Division Annual Report, July 1975-Sept. 1976," LA-6816-PR (Los Alamos Scientific Laboratory, Los Alamos, 1977), p. 147.

13. M. L. Sloan and W. E. Drummond, Phys. Rev. Lert. 25, 1129 (1970).

14. A. A. Irani and N. Rostoker, "Collective Acceieration of Ions by Inverse Cherenkov Effect in a Torus," UCI-TR-75-56 (University of California, Irvine, 1975). 
V. ACKNOWLEDGMENTS

V. Bailey and S. Putnam suggested this research and assisted in an early stage of it. R. J. Faehl, R. B. Miller, W. R. Shanahan, and L. E. Thode provided useful suggestions. We are indebted to thata.

This research was performed under the auspices of the U. S. Energy Research and Development Administration. Prior code development was supported in part by the Air Force Weapons Laboratory, Albuquerque. 


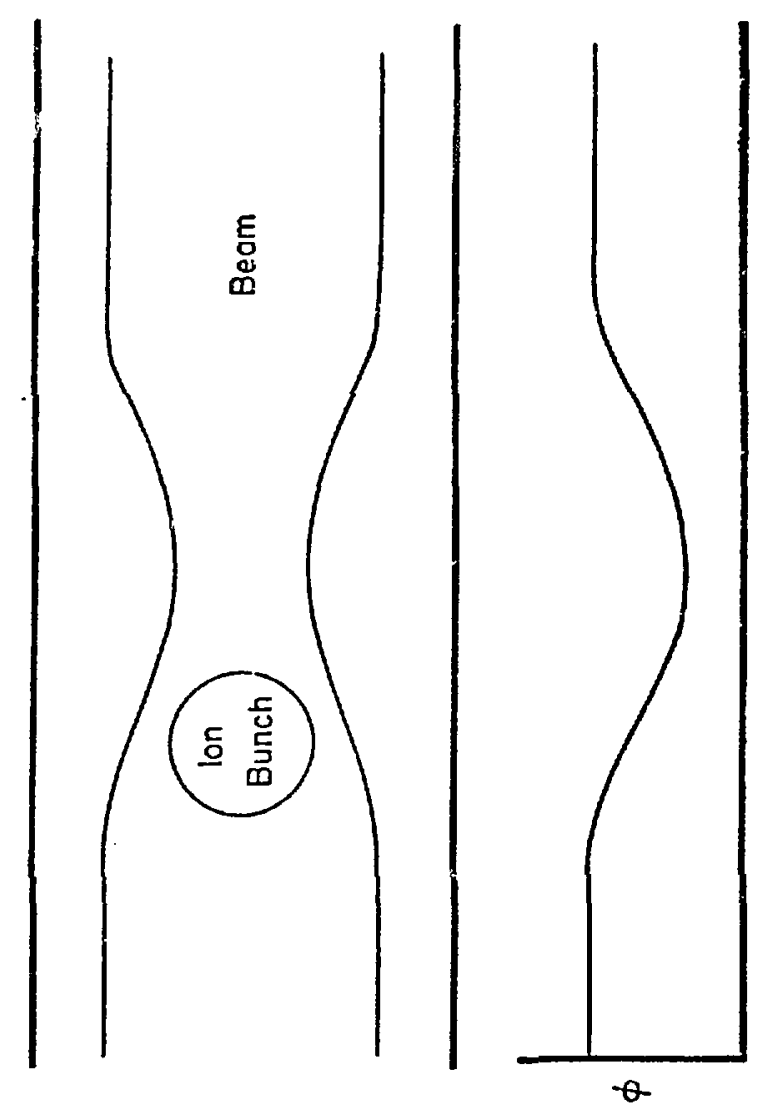

Fig. 1. Schematic of localized pinch model, showing beam (propagating to the right), ion bunch, pinch, and potential well.
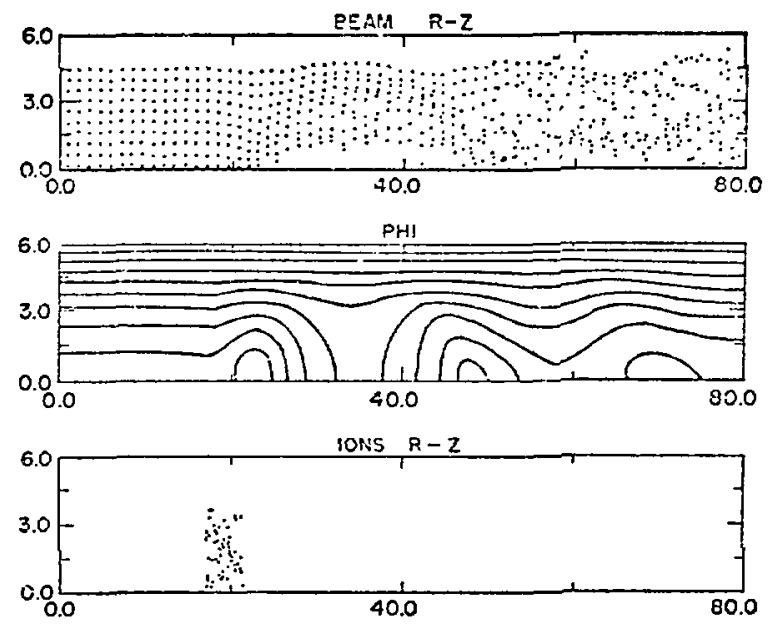

Fig. 3. Early tine behavior of $\gamma^{2} Q_{i} / Q_{e}=0.5$ ion bunch accelerating in a $V / Y=2$ electron beam. Frames, top to bottom, show positions of beam electrons, contours of the electrostatic potential, and positions of clump ions within the cylindrical guide tube.

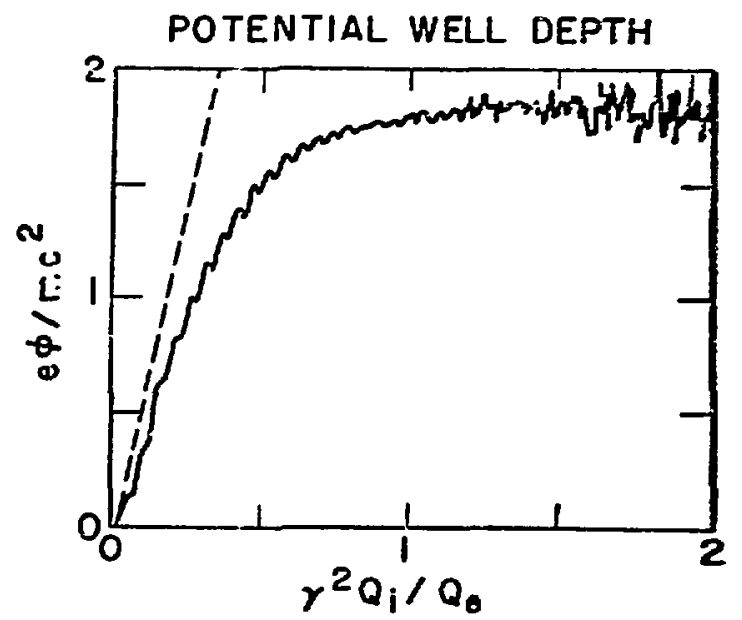

Fig. 2. Electrostatic well depth as a function of excess charge in the lon bunch for a $v=3$. $v / \gamma=2$, cold force neutral electron beam.
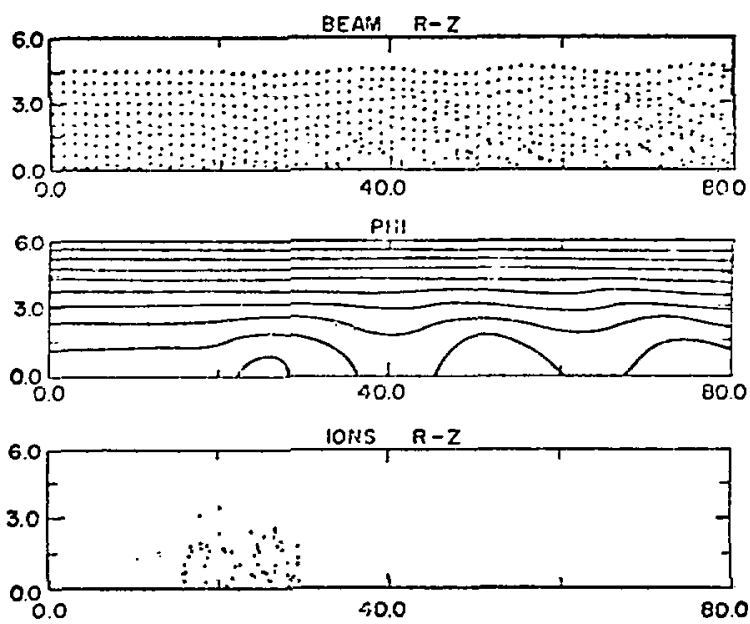

Fig. 4. Late time development of the simulation described in Fig. 3.

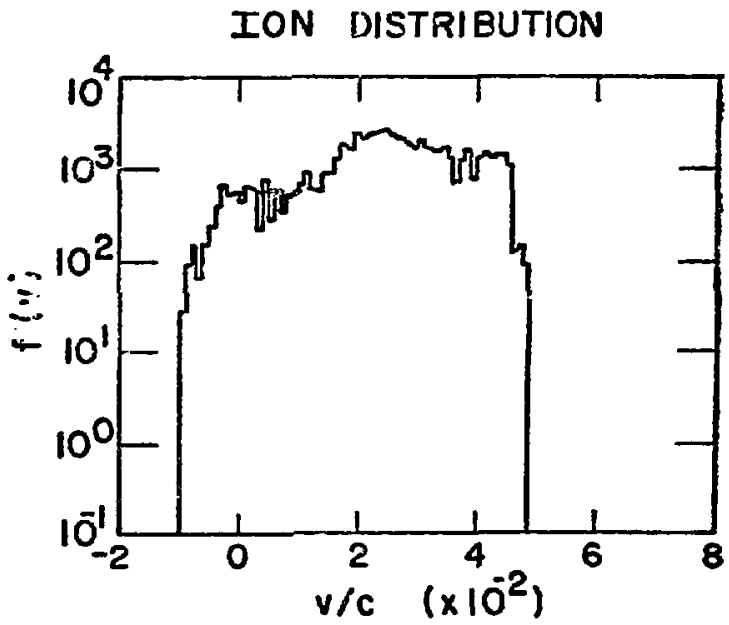

Fig. 5. Hydrogen ion longitudinal velocity distribution function for Fig. 4 . 
[Reprinted from IEEE Transactions or Nuclear Science 24, 1648-3650 (1977).]

APPENDIX L

COLLECTIVE ION ACCELERATION IN A TRAVELING VIRTUAL CATHODE 


\section{COLLECTIVF, ION ACCEIERATIOS IN A TRAVELI:G VIRTUAL CATHONEA}

R.B. Miller, R.J. Fachl, T.C. Genoni, and k'A. Proctor Air Force Weapons Laboratory, Kirtland AFB, New Mexico 87117

\section{Summary}

This paper describes a general class of coll cctive acceleration schemes wich rely on the controlled motion of a virtual cathode created in an intense relativistic electron beam. Preliminary numerical simulation results are presented for a specific scheme which does not depend on neutralization of the electron beam space charge. In particular, the position of virtual cathode formation appears to be adequately described by relatively simple equations. Also, electron beam turbulence due to a two-strean instability is shown to be substantially decreased by using a hollow beam and decreasing the external magnetic fiels strength.

\section{Incroduction}

The results of experiments at the Air Force lieapois Laboratory ${ }^{1, ?}$ performed with the low-pressure neutral gas ion acceleration configuration have indicated that the primary acceleration mechanism is the collapse of the deep potential well associated with the virtual cathode due to neutralization of the elertron space charge through ionization of the background gas. Since net acceleration occurs when the lons become untrapped, the process is largely uncontrolled and does not appear capable of generating large numbers of high energy ions.

A more desirable situation is that in which ions become trapped in a deep potential well and the motion of the well is then controlled to permit ion acceleration over substantial distances. In recent years, several acceleration schemes have been proposed to produce the desired goal. These schemes can generally be classified as ejther wave methods or net electron space-sharge methods. Examples of the first class include the autoresonant accelerator concept ${ }^{3}$ and the plasma wave scheme using a converging wave guide; an example of the second class is the controlled ionization front scheme of olson. ${ }^{5}$ More recently, a class E net space charge acceleration schemes has been proposed. ${ }^{8}$ " The basic concept which involves creating a single deep potential well (a virtual cathode) and controlling its motion, arose from a number of observations concerning virtual cathode formation in unneutralized beam propagation experiments. ${ }^{8}$ To summarize the significant features of these results: (1) the position of virtual cathode formation depends upon the ratio of the injected current to the space charge limiting current; and (2) this parameter varies over the electron beam pulse duration. It is therefore to be expected that the position of the virtual cathode should vary during the pulse, and it is speculated that by properly designing the drift tube geometry it should be posstble to use the time-dependent generator-diode behavior (essentially diode impedance) to create the conditions for the -ontrolled motion of a virtual cathode.

For a solid electron beam of radius $r_{b}$ and kinetic energy $\left(\gamma_{0}-1\right) \mathrm{mc}^{2}$ with fractional charge neutralization $f$ infucted into a long drift tube of radius $R$, the condition for virtual cathode formation is that the beam current exceed the space charge limiting current, i.e.,

$$
I(z, t) \geq I_{f}(z, t)=\frac{\left|\gamma_{o}^{\nu / 3}(z, t)-1\right|^{3 / 2} m c^{3} / e}{1+2 \ln \left[z(z, t) /\left.r_{b}(z, t)\right|^{\mid}\right.}\left|1-f_{\epsilon}(z, t)\right|^{-2} .
$$

It has been assumed that slow adiabatic variations in the quantities are allowed so that the argurent $(z, t)$ denotes the values of the quantities at the axial position $z$ and at $t$ ime $t$.

An examination of this equation indicates five parameters which can be varied either singly or in combination to prodice a virtual cathode whose position should vary with time.

(It should be roted that virtual cathode motion produced by variations in $f(z, t)$ is the essence of a particular embodiuent of olson's ionization front accelerator. ${ }^{5}$ In addition, it is believed that this mechanism (changing $f(z, t)$ ) is responsible for the ion acceleration observed in experiments performed by Luce, 9 and Greenwald, et al. ${ }^{10}$ )

As an example of changing combinations of variables to produce the desired virtual cathode motion, consider the case in which a beam of constant voltage and radius, but variable current, is injected into an evacuated drift tube whose radius varies as a function of axial position. In particular, for the situation in which the curreat rise is linear, i.e.,

$$
I(z, t)=\left\{\begin{array}{cl}
I_{0}\left(t-z / v_{e}\right) / t_{r} & ; 0 \leq\left(t-z / v_{e}\right) \leq t_{r} \\
0 & ; \text { otherwise }
\end{array}\right.
$$

where $I$ is the peak injected current, t is the current $r$ isetime, and is the electron velocity (assumed constant in $z$ and $t$. For the purpose of this example). it can be shown that the axial varlation in drift tube radius required for uniform acceleration of the virtual cathode toward the anode is given by ${ }^{6}$

$h(z)=h\left(z_{0}\right)+\left(\alpha v_{e} c^{\prime}\right)^{-1}\left\{\left[2 v_{e}^{2}\left(z_{0}-z\right) / a\right]^{1 / z}+\left(z_{0}-z\right)\right\}$

where

$$
\begin{aligned}
h(z) & =\left\{1+2 \ln \left[R(z) / x_{b}\right]\right\}^{-1} \\
\alpha & =\left(\gamma_{0}^{2 / 3}-1\right)^{3 / 2} m c^{3} / e 1_{0}
\end{aligned}
$$

$z$ and $t$ (the initial position of virtual cathode formation) àre related according to

$$
z_{0}=v_{e} t_{0}-\alpha v_{e} r^{h\left(z_{0}\right)}
$$

with a being the acceleration of the vircual cathode. An illustration of this cuncept is presented in Figure 1.

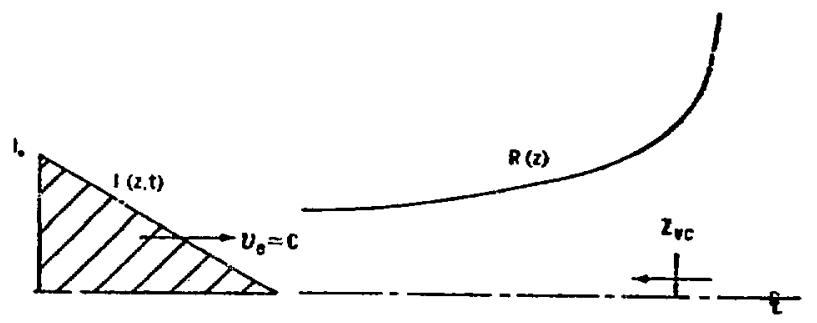

$$
z_{\mathrm{re}}=z_{1}-z_{\mathrm{a}}\left(\mathrm{t}-\mathrm{t}_{\mathrm{t}}\right)^{2}
$$

Figure 1. Schematic diagram of a moving virtual cathode resulting from time-dependent diode behavior and a flaring waveguide. 
Huch further analysis is ing usly requlred for this particular : cheve, in addition to several other sche-ies apparent from Eq. (1), but not considered here. of particular concern are: (1) the shape of the potent1al well; (2) the effects of varfations in electron beall velocity as the bean nears the virtual cathode region; (3) the effects of primary electrons reflected from the virtual cathode; and (4) the time dependent oscillations in well depth and postion around the quasi-static values. An examination of these questions as pertaining to this concept hos begun, supported in large part by nitmertcal simulations using the CCUBE particle code developed by Godfreg.' ' While the calculations are still in an early stage the prelininary results are of some interest.

\section{Numer 1cal Simulation Results}

The first problem to be addressed was the injection of a solid, constant current beai into a conical diverging waveguide for the purpose of examining the sultability of Eq. (1) for describing the position of virtual cathode formation. The configuration and the parameters used in the simulation are described in Figure 2 .

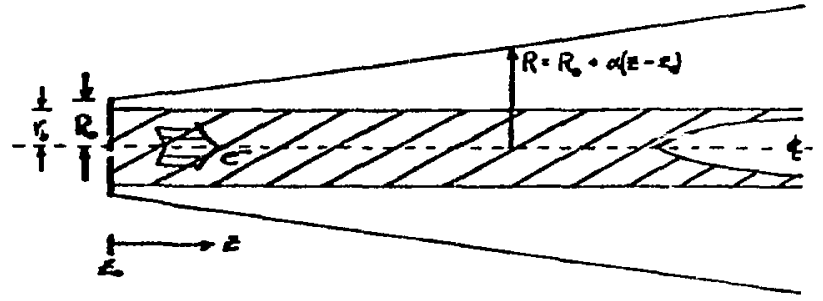

Figure 2

Schematic Geometry of the Numerical Simulations.

According to Eq. (1) for the parameter values used in the simulations, the virtual cathode should form at an axial position of approximately $\omega_{p} z / c=127$. In Fig. 3 the kinetic energy $(\gamma-1) m c^{2}$ of the beam particles is presented as a function of axial position in the drift tube. The virtual cathode $(\gamma-1=0)$ forms at an axial position of $\omega_{p} z / c=155$. Hence, it eppears that che interpolation expression, Eq. (1), underest imates the space charge limiting current in this instance by approximately $14 \%$.

The work of Voronin, et. al. ${ }^{12}$ has been recently extended to the case of a cylindrical drift cavity of length $L$ for annular beams with arbitrary radial dimensions. In the limit of a solid bean with $(R / L) \rightarrow$ 0 , a rigorous upper bound for the space charge limiting current is obtained as

$$
I_{l} \leqslant \frac{m c^{3}}{4 e} r_{b}^{2} \lambda\left(r_{0}^{2 / 3}-1\right)^{3 / 2}
$$

where $\lambda$ is the smallest eigenvalue that satisf les the boundary condition

$$
J_{0}\left(\lambda r_{b}\right)+\ln \left(r_{b} / R\right)\left(\lambda r_{b}\right) J_{1}\left(\lambda r_{b}\right)=0
$$

where $J_{0}$ and $J_{1}$ are the zero and first order Bessel functions of the first kind. Taking into account the slow axial variation in the drift tube radius, Eqs. (6) and (7) estimate that the maximum axial position at which the vircual cathode can initially form is $\omega_{\mathrm{p}} z / c=161$, in excellent agreenent with the simulation resuit. $t$
Good agreoment is also noted between the simulacton results and the prediction of the simple electrostatic waveguide theory whlch glves the potential depression (far from endplates or the virtual cathoce position) as

$$
t(r, z)=\frac{I}{B_{C}}\left[1-\left(r / r_{b}\right)^{2}+2 \ln \left(k(z) / r_{b}\right)\right]
$$

(The solid ines of Flp. 3 represent the extreme cases of $r=0$, and $r=r_{p}$ ) In addition, eridplate effects and the sha:p potentjal step on going from $I=I$, to $I>I_{i}$ (of order $\left(\gamma_{0} / 3_{-1}\right)$ are noted.

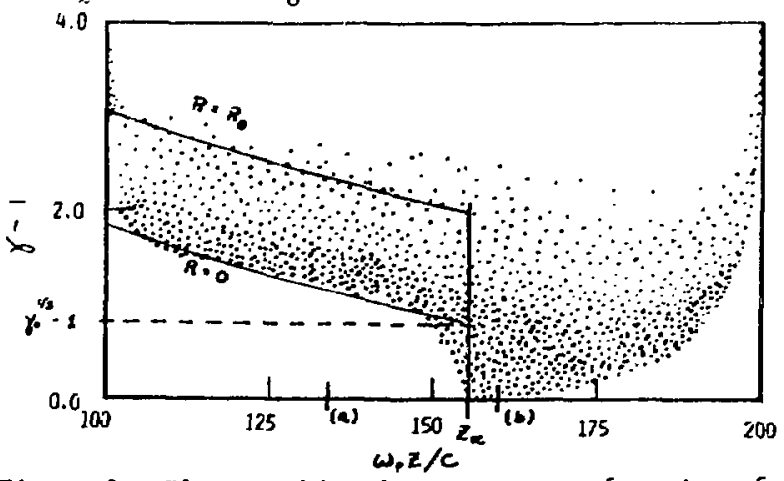

Figure 3. Electron kinetic energy as a function of axial position in the diverging conical waveguide. (a) estimated virtual cathode position as given by Eq. (1); (b) estimated upper bound for virtual cathode position as given by Eqs. (6) and (7).

Information concerning the evolution of the system of Fig. 2 can be obtained from the phase space diagram presented in Fig. 4 which exhibits longicudinal bean particle velocity as a function of longitudinal position in the waveguide.
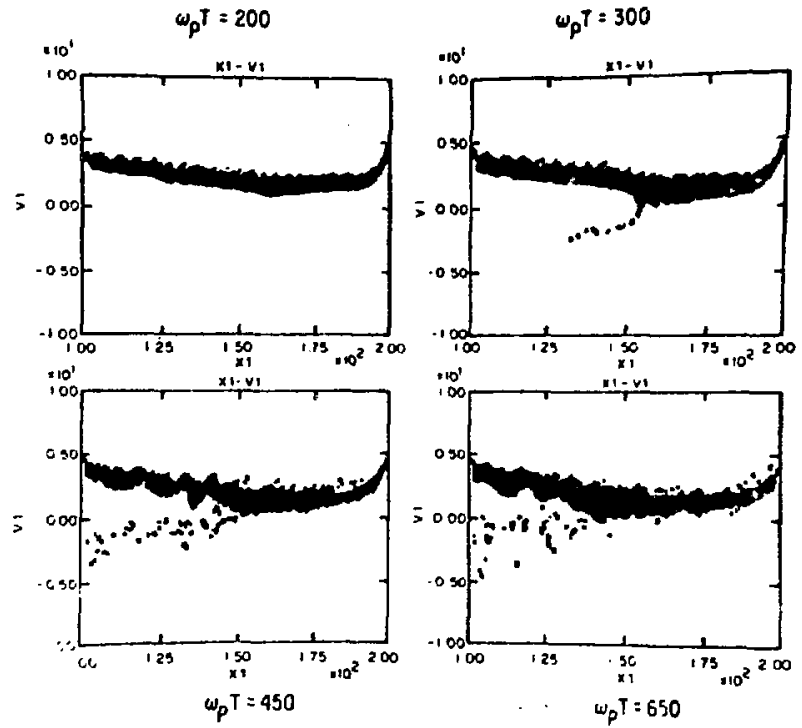

Figure 4. Evalution of longitudinal phase space.

$$
\left(z=x_{1}, v_{2}=v_{1}\right) \text {. }
$$

Early in the simulation $\left(\omega_{\mathrm{p}} \mathrm{T}=200\right)$ the magnitude of the electrostatic potential is increasing but virtual cathode formation $(v=0)$ has not occurred. By the time $\omega_{p} T=300$, the virtual cathode has formed, and reflected particles $\left(v_{1}<0\right)$ in the center of the beam are apparent. The free energy available as a

tApproximate analytical expressions recently derived by $J$. R. Thompson and M. L. Sloan also appear to give excellent agreement with the simulations. 
result of the streaming motion betwern the injerted and reflected particles results in the growth of a twostream instabllity $\left(\omega_{\mathrm{p}} \mathrm{T}=4.50\right)$. At late lines $\left(\omega_{\mathrm{p}} \mathrm{T}=\right.$ 650) the instability has vlolently distupted the heam and has essent lally destroyed the virtual cathode structure. These remarks are indicated more clearly in the late-time contour plots of potential and longitudinal electric field strength presented in Fig. 5. The iarge magnitude potential contours are very choppy and the electric fields are small.
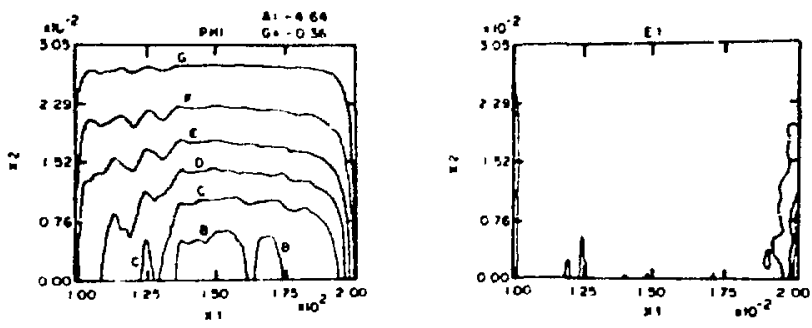

Figure 5. Solid beam potential and longitudinal electric field contours. $\omega_{p} T=625, \Omega_{c} / \omega_{p}=4.0$.

The problem of two-stream instability growth could be eliminated by having the reflected particles quickly pass to the wall of the drift tube. For the case of a solid beam, although the reflected particles did acquire a substantial radial component of velocity, the reflections occurred in the center of the beam. Consequently, the time over which the reflected particles could escape from the beam region was large, thereby giving rise to development of the catastrophic insta- bility. It was sperwlated that by using a thin hollow beam and decreaslag the external nagnet ic field strength this situation could be grtatly Improved. That this was indeed the case is shown in Fig. 6. For the hollow bean sinulatior, the reflected particles esrape from the bear $x$ egion across the decrlased magnetic field much more quickly. As a result, the virtual cathode structure (and the large accelerating fields) exists for wuch longer tires ( $\omega_{p} T=977$ for $\mathrm{F}[\mathrm{g}, 6)$.

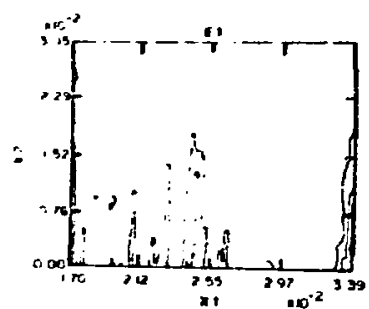

Figure 6. Hollow beam potential and longitudinal electric field contours. $\omega_{p} T=977, \Omega_{c} / \omega_{p}=2.0$.

Further simulation work will investigate the motion of the virtual cathode induced by a programed increase In the injected current as a function of tiwe. In addition, it should be noted that, as presented, the scheme indicated in Fig. I is more sultable for accelerat ing negative ions (or possibly electrons). The feasibility of accelerating positfve lons will be further investigated. Finally, experimental results pertalning to this scheme should soon be forthcoming.

\section{References}

1. R. B. Mller and D. C. Straw, J, Appl, Phys, 47, 1897 (1976).

2. D. C. Straw and R. B. Mfller, J. Appl. Phys, 47, $4681(1976)$.

3. M. L. Slcan and W. $\Sigma$. Drummond, Phys. Rev. Lett. 31 , 1234 (1973).

4. P. Sprangle, A. T. Drobot, and W. M. Mannheimer, Phys. Rev. Lett. 36, 1180 (1976).

5. C. L. 01son, Proc. IX Int' 1 Conf. High Energy Accelerators, SLAC (1974).

6. R. B. Miller, Air Force Weapons Laboratory Tech. Rept. No. AFWL-DTS-TN-75-115, (Unpublished).

7. R. B. Miller, IExE Proc. Int'1 Conf. Plasma Science, 130, Mar 24-26, 1976.

8. R. B. Miller and D. C. Straw, J. Appl. Phys., 48 (March 1977).

9. J. S. Luce, Ann. R. Y. Acad. Sc1., Vol 251, 217 (1975).

10. A. Greenwald, R. Lowell and R. Litele, Bull. Am. Phys. Soc. 21, 1147 (1976).

11. B, B. Godfrey, J, Comp. Phys. 19, 58 (1975).

12. V. S. Voronin, Yu. T. Zozulya and A. N. Lebedev, Sov. Phys. Tech. Phys. 17, 432 (1972).

*This research was supported in part by the Air Force Office of Sclentific Research 
[Submitted for publication.]

\author{
APPENDIX M \\ COLLECTIVE ION ACCELERATION THROUGH TEMPORAL MODULATION OF \\ RELATIVISTIC ELECTRON BEAM ENERGY \\ R. J. Faehl and B. B. Godfrey
}

\begin{abstract}
A new method is proposed for collectively accelerating bunches of ions with an intense relativistic electron beam. By varying the beam energy in time, acceleration of a slow doppler-shifted cyclotron wave can be accomplished, without accompanying degradation of the beam equilibrium. Test particle calculations employing this acceleration scheme indicate performance which is highly competitive with other collective ion acceleration mechanisms.
\end{abstract}


The possibility of using relativistic electron beams to collectively accelurate ions has sparked considerable interest recently. ${ }^{1-7}$ Among the most promising concepts is that of a traveling wave accelerator employing the self fields of the beam itself. ${ }^{1,2}$ In previous proposals, wave acceleration was induced by a weak spatial inhomogeneity in either the external guide fields or the geometry, with time independent beam and field conditions. In contrast we propose to accelerate a slow dopplershifted cyclotron wave by temporally varying the beam energy in an axially homogeneous vacuum drift tube. The cyclotron wave is deemed most appropriate of the eight beam eigenmodes because its phase velocity can be varied from near zero to almost the speed of light and because of its moderately strong dependence on beam energy. Among the advantages for this type of acceleration are that it leads to less attenuation of the wave fields during the acceleration process, the beam equilibrium is altered less by energy change than by either field or geometry variations, and only simple cylindrical geometry is required.

All accelerator configurations must possess tro general characteristics, large fields and the ability to move the fields so that synchronism with the particles is maintained. Conventional accelerators are limited to fields on the order $4-5 \times 10^{4} \mathrm{~V} / \mathrm{cm}$. Fields of $10^{6} \mathrm{v} / \mathrm{cm}$ or better, however, appear accessible with present relativistic electron beams. Development of higher energy beams, moreover, will facilitate generation of even stronger fields. limitations at these very high field strengths may come from field-induced breakdown of chamber materials rather than plasma phenomena. The central problem in using intense relativistic beams, therefore, is in devising controllable methods for moving the field. 
Eigenmodes of the electron beam are particularly attractive for collective ion acceleration because of their known propagation characteristics, stability, and periodic field structure. The last of these tends to enforce synchronism of ions. Schemes which use zero-order fields, such as virtual cathodes $^{3,4,7}$ or localized pinches, ${ }^{5}$ produce strong fields but are difficul. to control. Previous traveling wave collective acceleration schemes ${ }^{1,2}$ do employ eigenmodes, but have relied on weak spatial inhomogeneities to increase the phase velocity of the waves. Weak inhomogeneity is required so that the homngeneous dispersion relation is valid locally. The machematical description of the waves is therefore derived from a WKE analysis. Since the conditions are all steady, the rrequency is invariant. Wavelength variations are thus responsible for the increase in phase velocity, $\mathrm{V}_{\mathrm{ph}}=\frac{\omega}{\mathrm{k}}$ The wave fields must remain large enough to trap the ions, however, and this field varies as $E_{z} \sim\left(k-\frac{\omega}{c}\right) \phi$. Hence, the trapping field is decreased during the acceleration. To avoid losing ions from the potential we11, the wave acceleration must be decreased and the length of the accelerator increased. Since the main economics from collective ion acceleration come from reductions in accelerator length, this is not desirable.

A conceptually different means of inducing wave acceleration arises when the beam energy is temporally modulated. If the modulation is caused at the diode, the simnlest way, the perturbations propagate with the beam at $v_{0}=c \frac{\left(\gamma^{2}-1\right)^{1 / 2}}{\gamma}$ The wave phase velocity is usually much less than $v_{0}$, however, so the wave "sees" an almost uniform change in the nedium. For example, a plane wave phase function is $\psi_{0}=k_{0} z-\omega_{0} t$. An infinitesimal variation of the wavenumber, $k=k_{0}-\delta k$, can give an arbitrarily large phase change as $z \rightarrow \infty$. The entire wavetrain has simply dilated. 
Near the front edge of the modulation, the phase velocity, which is the trajectory of a point of constant phase, can then approach aibitrarily close to $v_{0}$, as $z \rightarrow \infty$. The rate at which this wave velocity increases is governed by the rate of change of the beam energy, $\varepsilon=m_{0} c^{2}(\gamma-1)$, and not by the magnitude of the change. Thus, in a sufficiently long system, one can envision ion acceleration :o very high energies with only modest variations in $y$.

The goal of collective ion acceleration is to reduce accelerator length, of course, not to make it arbitrarily long. To quantify the above concepts, we must first derive an appropriate phase function. The main characteristics of the beam cyclotron wave can be derived by considering the $\mathrm{r}$ and $\theta$ components of the cold fluid equations. The properties of cyclotron vave propagation are easily calculated from the phase function.

Consider a cold cylindrical electron beam propagating along the z-axis. For an axisymmetric wave $(m=0)$, neglect of diamagnetic effects leads to

$$
\begin{aligned}
& \frac{\partial p_{r}}{\partial t}+v_{o} \frac{\partial p_{r}}{\partial z}-\frac{v_{\theta} p_{\theta}}{r}=-\frac{e}{m} E_{r}-\frac{e}{m c \gamma} p_{\theta} B_{z}+\frac{e}{m c} v_{o} B_{\theta}, \\
& \frac{\partial p_{\theta}}{\partial t}+v_{0} \frac{\partial p_{\theta}}{\partial z}+\frac{v_{\theta} p_{r}}{r}=\frac{e}{m c \gamma} p_{r}{ }^{B}
\end{aligned}
$$

where $p_{i}=\gamma v_{i}$ and $\gamma \doteq\left[1-\left(\frac{v_{o}}{c}\right)^{2}\right]^{-i / 2}$. Since we expect an equilibrium rotation, let $v_{\theta}=v_{\theta}^{R}+\widetilde{v}_{\theta}$. The average rotational velocity is

$$
v_{e}^{R} \doteq-\frac{\Omega_{o}}{2 \gamma}\left[1 \pm\left(1-2 \frac{\bar{\omega}_{p}^{2}}{\gamma \Omega_{o}^{2}}\right)^{1 / 2}\right] r
$$

where $\Omega_{o}=\frac{e z_{z}}{m c}, \overline{u s}_{p}^{2}=\frac{4 \pi \bar{n}_{e} e^{2}}{m}$, and $\bar{n}_{e}=\int_{0}^{R} n_{e} d r / R$. In the slow rotation mode 


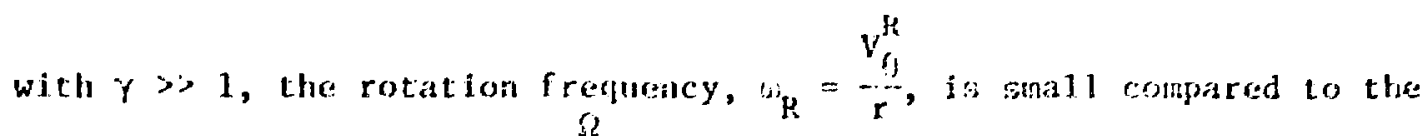
relativistic gyrofrequency, $\frac{\Omega}{r}$. It furthermore becomes proportionately smaller with increasing $\gamma$. Its neglect makes the key piysics more transparent without seriousiy effecting any results. Combining the remainder of (1a) and (1b) gives

$$
\left(\frac{\partial^{2}}{\partial t^{2}}+2 v_{0} \frac{\partial^{2}}{\partial t d z}+v_{0}^{2} \frac{\partial^{2}}{\partial z^{2}}\right) \tilde{\mathrm{p}}_{r}+\left(\begin{array}{c}
\Omega_{0} \\
\gamma
\end{array}\right)^{2} \ddot{\mathrm{p}}_{\mathrm{r}}=0
$$

where the assumption that $\gamma \gg 1$ makes $v_{0}$ such a weak function of $\gamma$ that we treat it as a constant. For homogeneous steady conditions, gives the dispersion relation

$$
\omega_{0}=k_{0} v_{0} \pm \frac{\Omega_{0}}{\gamma_{0}}
$$

A more accurate electromagnetic analysis gives 8

$$
\omega_{0}=k_{0} v_{0} \pm \frac{\Omega_{0}}{\gamma_{0}} \frac{\left(k_{o}^{2}+k_{1}^{2}\right) c^{2}}{\left(k_{0}^{2}+k_{1}^{2}\right) c^{2}+\omega_{p}^{2} / \gamma_{0}},
$$

but in the $\gamma_{0} \gg 1$ limit (4) is an excellent approximation. Equation (3) is, therefore, a reasonably accurate equation for describing cyclotron wave propagation. If we now express $\tilde{\mathrm{p}}_{\mathrm{r}}$ in terms of amplitude and phase functions,

$$
\tilde{p}_{r}=A(z, t) e^{i \psi(z, t)}
$$

we can derive a simple equation for $\psi$ in the slowly varying amplitude approximation 


$$
\left(\frac{\partial}{\partial t}+v_{0} \frac{\partial}{\partial z}\right):(z, t)= \pm \frac{\zeta}{y}
$$

where the + sign is appropriate here. (This corresponds to $u=k v_{0}-\frac{\Omega_{0}}{\gamma}$ ) If $y$ is a function only $c i \quad i=t-\frac{z}{v_{0}}$, the transformation to $(n, c)$ variables, $\xi_{s}=t+\frac{z}{v_{0}}$, permits a general solution to (6)

$$
\psi(n, \xi)=-\frac{1}{2} \frac{\eta}{\gamma(r)} \tilde{r}+c(n)
$$

provided $B_{z}$ is independent of $\xi$. To evaluate the function $C(r)$ we need boundary conditions based on physical considerations.

A particularly interesting boundary condition arises if a monochromatic wave of frequency $\omega_{0}$ is launched at $z=0$. This might be accomplished with a simple loop antenna, well removed from the beam diode. Then the condition at $z=0$ is simply

$$
\psi(0, t)=-\omega_{0} t_{0}
$$

Without specifying $\gamma\left(t-\frac{z}{v_{0}}\right)$, we can evaluate $(7)$, which leads to

$$
\psi(z, t)=k_{0} z-\omega_{0} t-\frac{\Omega_{0}}{\gamma_{0}}\left(1-\frac{\gamma_{0}}{\gamma}\right) \frac{z}{v_{0}}
$$

where we have used (4) as a formal definition of $\omega_{0}$. The phase velocity corresponds to a point of constant phase, or $\frac{\mathrm{d} \psi}{\mathrm{d} t}=0$. Thus the general expression for phase velocity, with condition (8), is

$$
v_{p h}=\frac{\omega_{0}-\frac{\Omega_{0}}{\gamma_{0}} \frac{z}{v_{0}} \frac{\partial}{\partial \eta}\left(\frac{\gamma_{0}}{\gamma(n)}\right)}{k_{0}-\frac{\Omega_{0}}{\gamma_{0} v_{0}}\left[1-\frac{\gamma_{0}}{\gamma(n)}+\frac{z}{v_{0}} \frac{\partial}{\partial \eta}\left(\frac{\gamma_{0}}{\gamma(n)}\right)\right]}
$$


For $\frac{\partial}{\partial n}\left(\frac{1}{\gamma}\right)<0,(10)$ indicates that the phase velocity of a cuclotron wayce can be increased, at a rate dependent on the pulsestape of the beasenergy modulation and the distance from tine emitter.

The wave acceleration described by (10) differs fundamentally from that induced by spatial modulation. With the latter, the wavelength of a constant frequency disturbance dilates as a function of a:ial displacement. Since $v_{p h}=w / k$, the phase velocity increases proportionately, but the trapping field, $E_{t r} \times k-w / c$, also suffers a corresponding decrease. By energy modulating the electron beam, on the other hand, phase changes propagated down the wavetrain at $v_{0}$ alter both the local wavelength and frequency. Thiss ameliorates the degradation of the trapping fields. So long as $v_{0} \gg v_{p h}$, the result is a nearly uniform wavetrain dilation. Although a lag exists due to the finite propagation velocity of the modulations, the basic picture remains valid. A sma]1 change in beam energy can result in a very large velocity change at large displacements. In both spatiai and temporal modulation, self-consistent acceleration of traveling waves must degrade the trapping fields. By increasing the frequency $=s$ we11 as the wavelength, however, it is easy to show that temporal modulation leads to less degradation.

The requirement that particles remain trapped implies that the trapping field must be at least as large as the accelerating field. This criterion can be translated into a condition for maximizing the effective acceleration,

$$
|a|=\frac{q}{M_{i}}\left(k-\frac{\omega}{c}\right) \phi_{0}
$$

where $k \equiv \frac{\partial \psi}{\partial z}, \omega \equiv-\frac{\partial \psi}{\partial t}$, and a, the wave acceleration, is derived from the condition $\frac{\mathrm{d}^{2} \psi}{\mathrm{d} t^{2}}=0$. The functional dependence of $\gamma$ for maximum average 
acceleration comes from the solution of (11), which, howerer, is an excecdingly nonlincar equation. Numerical solutions corresponding to (11), to constant acceleration, and to various analytic forms for $Y$ will be presented in a more complete article. For the present purposes, we will exhibit only a simple numerical example to illustrate the parameters one might expect in an accelerator based on this method.

Despite arguments based on comparison of wave field to wave acceleration, one must ultimately solve the ion equation of motion

$$
\frac{d \vec{p}_{i}}{d t}=\frac{Z e}{M_{i}} E_{0} \sin b,
$$

where $\bar{p}_{i}=\gamma_{i} \bar{v}_{i}$, and $F_{0}=\left(k-\frac{w}{c}\right) \phi_{0}$. We have numerically solved (12) for an ensemble of test ions. The test particles were given the same initial velocity, and uniformly spaced over one cyclotron wavelength. The wave was assumed to maintain a constant potential, although there is uncertainty about this point. Higher beam energy would facilitate growth to larger potential, but there is at present no good method for gauging the magnitude of potential change with $y$-variation. For an initial wave field of $10^{6} \mathrm{~V} / \mathrm{cm}$, and a linear modulation of the energy, $\gamma=\gamma_{0}\left(1+\frac{n}{\tau}\right)$, protons initially at $8.5 \mathrm{MeV}$ were accelerated to over $150 \mathrm{MeV}$ in a distance of $380 \mathrm{~cm}$, when the beam energy was increased from $3 \mathrm{MeV}$ to $18 \mathrm{MeV}$. This corresponds to an average accelerating field of over $3.8 \times 10^{5} \mathrm{~V} / \mathrm{cm}$, which is comparable with $t^{\prime} e$ expectations of other traveling wave schemes and almost an order of magnitude better than conventional accelerators. Velocity distributions of the test particles at $t=0, \tau$, and $5 \tau$ are shown in Figure $r$. Although significant detrapping has occurred by $t=5 \tau$, most particles with $v>.5 c$ were accelerated later to $v>.60 c$. Optimized energy forms will permit further reductions in accelerator length. 
Hosit of the requirements needed for this method of ion acceleration are elther within current technology or within foresceable extensions of 1t. Intense relativistic clectron beams of 3-5 $\mathrm{MeV}$, for instance, are cornmonplace, while intense beams of $30-50$ lev are not. The fact that $\gamma: 20$ machines have been built, however, suggests that higher energy machines might be feasible, if sufficient incentive were available. ${ }^{9}$ The ability to control the energy of the bean in time is not the simplast requirement on an electron beam machine, but may be feasible throw design of a multielement accelerator or decelerator downstream from the ordinary diode. 10

A more fundamental 1 imitation of this method concerns acceleration to velucities close to $\mathrm{c}$. As mentioned above, there is a lag between introduction of a modulation and the time it reaches the particles, due to finite propagation speed. For $v_{p h} \sim .5 \mathrm{c}$, one can imagine the waves almost outrunning the modulation. Of course, the "kick" from the modulation eventually does catch up, but the additional distance traversed by the particles in the interim has the effect of weakening the average accelerating field. For these high velocities, therefore, it may be mare efficient to use other collective acceleration schemes, some of which, in fact, are inefficient at low velocity. ${ }^{11}$ In other applications, ion energies of 100-200 MeV may be acceptable as they are. Finally, even though high velocities may be inaccessible, the use of higher atomic number ions, such as in heavy ion fusion, would still permit generation of significant ion energies, $10-20 \mathrm{GeV}$ for $\mathrm{A}=200$, in very modest length accelerators.

There are still many unanswered questions concerning the effect of energy modulations on collective plasma effects. Preliminary studies indicate that the eigenmodes of the cyclotron waves are not seriously 
effected by this, but these maters require more detailed analytic and numerical studies. These studies are in progress, as well as studies into the effect of energy morlulation on other beam modes.

Ackncwledgements. We would like to thank Dr. B. S. Newberger for useful discussions on this subject. 


\section{HI BL.IOGRAYHY}

1. M. L. Sloan and W. F. Urumnond, Phys. Rev. Letters 31, 1234 (1973).

2. P. Sprangle, W. M. Hanheimer, and $\Lambda$. T. Drobot, Phys. Rev. Letters 36, $1180(1976)$.

3. C. I. OJson, Phys. Rev. A 11, 2631 (1974).

4. C. L. Olson, Particle Acceleratcrs 6, 197 (1975).

5. S. Putnam, Phys, Rev. Letters 25,1129 (1970).

6. A. V. Agafonov, A. A. Kolomenskii, and I. I. Logochev, Sov. Phys. JETP Letters 22, 231 (1975).

7. R. B. Miller, R. J. Faeh1, T. C. Genoni, and W. A. Pro.tor, IEEE Transactions on Nuclear Science, 24, 1948 (1977).

8. W. E. Drummond, G. I. Bourianoff, D. E. Hasti, W. W. Rienstra, M. L. Sloan, and J. R. Thompson, AFWL-TR-74-343 (1975).

9. D. C. Straw (Private Communication).

10. A. I. Pavlovskii, V. S. Bosarnvkin, G. D. Kuleshov, A. I. Gerasimov, V. A. Tananakin, and A. P. Klement'ev, Sov. Phys. Dok1. 20, 441 (1975).

11. B. B. Godfrey, IEEE Transactions on Plasma Science, (accepted for publication). 
$1(a)$

$1(b)$

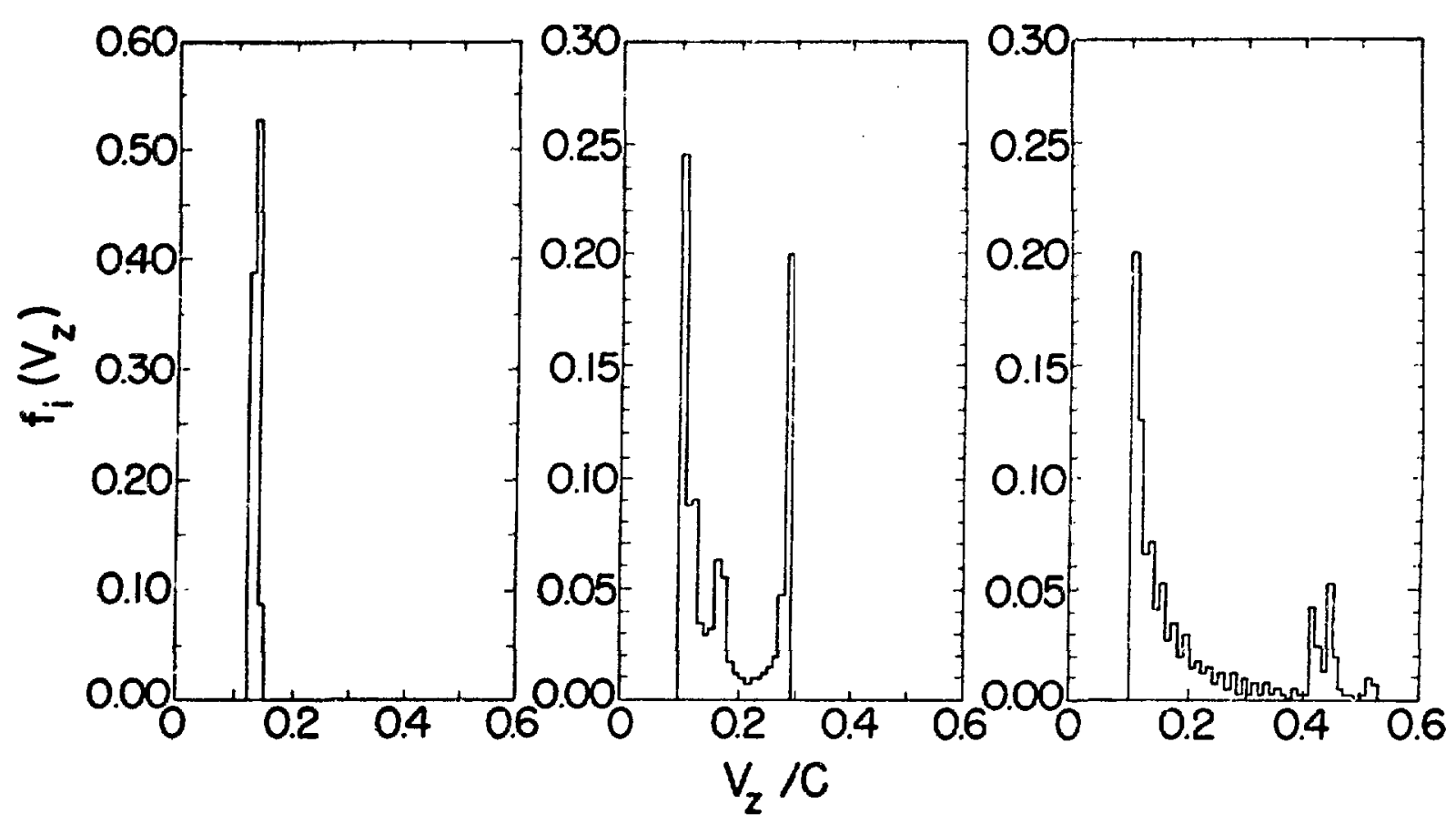

Fig. 1. Velocity distributions of 400 test particles initially at $v=0.13 \mathrm{c}$. Beam energy was varied as $\gamma=\gamma_{0}\left[1+\left(t-z / v_{0}\right) / \tau\right]$. Distributions plotted at $(a) t=0,(b) t=t$, (c) $t=5 T$. Peak energy at $t=5 i$ was $164 \mathrm{MeV}$.

277 
[Internal report, 1977.$]$

APPENDIX N

INITIAL APPROACHES TO A 3-D LINEAR SIMULATION CODE

Barry S. Newberger

Here we will briefly describe our efforts in the development of a three-dimensiona? time-dependent linear plasma simulation code. This code will be used to augnent the results of CCUBE in part and perhaps look at the stability of other equilibria in their own right. The former application wili be useful in answering the question whether twodimensional equilibria (non-linear saturated normal modes) will be unstable to motions which do not have a symmetry axis.

Our initial efforts were in the direction of a linear PIC code and was the most general (1 inear) scheme which could be used insofar as the particle distribution could be modeled directly. The PIC approach had to be abandoned however. In analytic calculations, 1 inearization of the model equations typically results in a major simplification of the work in part because the techniques available for the solution of linear differential equations are more extensive than those for nonlinear DE's and, in many cases, fourier analysis is available to use. This simplification does not hold up in numerical simulation models. Here we are constrained by considerations of storage and running time. Although methods such as fourier analysis in the cyclic coordinate in 2-D hold up, this now introduces complex quaritities and storage requirements double, for example.

Two PIC methods were investigated, the first solved for the perturbed distribution function by integrating the Vlasov equation along zero. order orbits. The first order distribution function is given by 


$$
\tilde{f}-\tilde{f}(0)=-\frac{1}{m} \int_{0}^{t} \tilde{F}_{j} \frac{\partial f_{0}}{\partial u_{j}} d t^{\prime},
$$

where $\tilde{F}_{j}$ is the first order force and fo the zero order distribution function. The charge and current density are calculated from the zero and first mornents of $E q$. (1)

$$
\delta j_{i}(t)=\int \frac{u_{i}}{\gamma} \tilde{f}(0) d^{3} u-\frac{1}{m} \int_{0}^{t} \int \frac{u_{i}}{\gamma} \widetilde{F}_{j} \frac{\partial f}{\partial u_{j}} d^{3} u d t^{\prime}
$$

and similarly for on. In order to eliminate the derivative of the zero order distribution function which is typically undesirable numerically, one integrates by parts. In this case, since the functions appearing in the integrand are evaluated along zero-order orbits, $\tilde{F}_{j}$ has an implicit dependence on the velocity and this term must be included in salculating $\delta j_{j}$ and $\delta n$. Numerically, it leads to an evaluation of a series of terms 2 in length at the $\ell$ 'th time step.

Another PIC approach is to integrate the equations of motion directly. After fourier analyzing in the ignorable coordinate, the equations of motion become

$$
\frac{d \hat{u}_{j}}{d t}-i k v_{3}^{0} \hat{u}_{j}^{l}=\frac{e}{m} \hat{F}_{j}^{l}+\left(\hat{x}_{i} \cdot \vec{F}_{0}\right)_{j}
$$

and

$$
\frac{d \hat{x}_{j}^{l}}{d t}-i k v_{3}^{0} \hat{x}_{j}^{\}}=\frac{1}{\gamma_{0}}\left(\hat{u}_{j}^{\prime}-\frac{u_{j}^{0}}{\gamma_{0}^{2}} \sum_{i} u_{i}^{0} \hat{u}_{i}^{l}\right)
$$


where the carat denotes a (complex) fourier amplitude. The difficulty enters here in differencina these equations. Although these were derived by linearizing the full equations of motion, not ali difference schemes will preserve the inearity, and approximate solutions computed from one of these schemes would be meaningless. A scheme was derived by Freidberg, et al. 1 and implemented in a one-dimensional electrostatic code. This "linearity preserving" scheme amounts to higher order interpolation and storage in higher dimension systems would be prohibitive.

Furthermore, there are questions relating to the number of particles per cell required in a given linear simulation, the answers to which have an obvious bearing on the running time of a particular problem. It appears, therefore, that linearization in numerical PIC simulation may not buy anything and the full nonlinear simulation may be less costly than the linear one, particularly in a system where only a few order modes in the third dimension are potentially dangerous. In this case, relatively few zones in the third dimension may be necessary and storage requirements may not be much worse than the 2-D case, but nevertheless this is not an alternative until an operating system on the Cray is established. In the light of these considerations, we abandoned a linear PIC scheme as not viable.

We have now turned our attention to a hydrodynamic approach. Here the problems of storage are not so acute, but algorithms for numerically integrating the fluid equations now become important. The Maxwells equations can be center differenced in space and time in a straightforward way but the fluid equations cannot. Methods for handling this 
part of the froblem that are stable, accurate and not too dissipative must be found. Two metriods under consideration at the present time are the method of characteristics and a predictor-corrector scheme.

Although the fluid mode? is less versatile than PIC, it should be able to provide answers to the critical questions regarding stability of the 2-0 non-linear "equilibria" until the time when a fully 3-D simulation code is practical.

\section{REFERENCES}

1. J. P. Freidberg, R. L. Morse, and C. W. Neilson, Proc. 3rd Annual Nunerical Plasma Simulation Conference, Stanford 1969, Paper 15. 